\title{
Remote Medical Monitoring Decision Support System and User Interface Usability
}

\author{
By \\ Anneliis Tosine, B. Eng. \\ A thesis submitted to \\ The Faculty of Graduate Studies and Research \\ in partial fulfilment of \\ the degree requirements of \\ Master of Applied Science in Biomedical Engineering \\ Ottawa-Carleton Institute for Biomedical Engineering (OCIBME) \\ Department of Systems and Computer Engineering \\ Carleton University \\ Ottawa, Ontario, Canada
}

October 2010

(C) Copyright 2010, Anneliis Tosine 


$\begin{array}{ll}\begin{array}{l}\text { Library and Archives } \\ \text { Canada }\end{array} & \begin{array}{l}\text { Bibliothèque et } \\ \text { Archives Canada }\end{array} \\ \begin{array}{l}\text { Published Heritage } \\ \text { Branch }\end{array} & \begin{array}{l}\text { Direction du } \\ \text { Patrimoine de l'édition }\end{array} \\ \begin{array}{l}\text { 395 Wellington Street } \\ \text { Ottawa ON K1A ON4 } \\ \text { Canada }\end{array} & \begin{array}{l}395, \text { rue Wellington } \\ \text { Ottawa ON K1A ON4 } \\ \text { Canada }\end{array}\end{array}$

Your file Votre reférence

ISBN: 978-0-494-79553-8

Our file Notre référence

ISBN: 978-0-494-79553-8

NOTICE:

The author has granted a nonexclusive license allowing Library and Archives Canada to reproduce, publish, archive, preserve, conserve, communicate to the public by telecommunication or on the Internet, loan, distribute and sell theses worldwide, for commercial or noncommercial purposes, in microform, paper, electronic and/or any other formats.

The author retains copyright ownership and moral rights in this thesis. Neither the thesis nor substantial extracts from it may be printed or otherwise reproduced without the author's permission.
AVIS:

L'auteur a accordé une licence non exclusive permettant à la Bibliothèque et Archives Canada de reproduire, publier, archiver, sauvegarder, conserver, transmettre au public par télécommunication ou par l'Internet, prêter, distribuer et vendre des thèses partout dans le monde, à des fins commerciales ou autres, sur support microforme, papier, électronique et/ou autres formats.

L'auteur conserve la propriété du droit d'auteur et des droits moraux qui protège cette thèse. Ni la thèse ni des extraits substantiels de celle-ci ne doivent être imprimés ou autrement reproduits sans son autorisation.
In compliance with the Canadian Privacy Act some supporting forms may have been removed from this thesis.

While these forms may be included in the document page count, their removal does not represent any loss of content from the thesis.
Conformément à la loi canadienne sur la protection de la vie privée, quelques formulaires secondaires ont été enlevés de cette thèse.

Bien que ces formulaires aient inclus dans la pagination, il n'y aura aucun contenu manquant. 


\section{Abstract}

User requirements were gathered from home care clinicians to understand what parameters are critical in monitoring the health of home care clients. Once solicited, the most appropriate graphical user interface (GUI) features were determined in order to conduct a usability test and qualitative analysis of two GUI prototypes. A few key findings include the need to display data trends, client personal targets and alerts for emergent situations.

Visual data mining combines data visualization and data mining. Therefore, in order to populate the GUI with home care clients' data and support decision making, data mining was also explored. Two data mining techniques, a segmentation algorithm and a feed-forward neural network, were evaluated for their ability to detect trends from simulated data. Results indicate that the segmentation algorithm is more accurate with the given data sets but the network is more robust with varying levels of noise. 


\section{Acknowledgments}

First, I would like to thank my co-supervisors, Dr Rafik Goubran and Dr Avi Parush for their guidance, wisdom, and patience for taking the risk of allowing me to work across the departments of Psychology and Systems and Computer Engineering. It was very important for me to accomplish multi-disciplinary research in the fields of biomedical and human factors engineering and I truly appreciated the opportunity to do so at Carleton.

Second I would like to recognize the financial support for this research, which has been provided by the Natural Sciences and Engineering Research Council of Canada (NSERC) and Carleton University.

I would also like to express my gratitude to the co-investigators and collaborators of the project: "Integration of bio-physiological information with a point-of-care decision support system for safer patient care".

Lastly, I would like to thank my friends and family for their unwavering support with my research. I would specifically like to acknowledge the encouragement from my parents who have supported me with every decision I have made and without them, I would not have been nearly as successful. I would also like to mention the patience and kindness of my sister, Katrin Tosine, who continuously drives me to do better and Colin Goodwin, for believing in me every small step of the way and for always cheering me on. Thank you. I could not have done it alone. 


\section{Table of Contents}

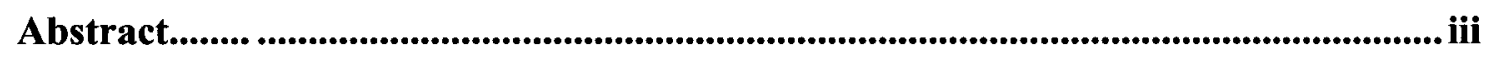

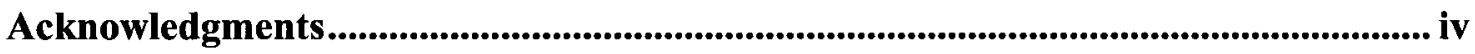

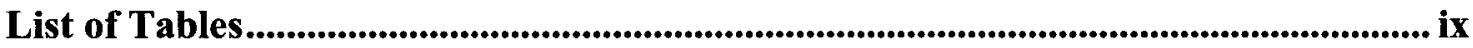

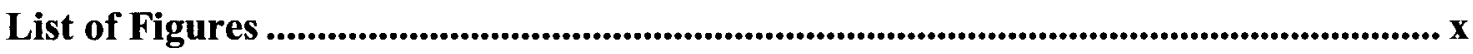

Chapter 1 Introduction ............................................................................................... 1

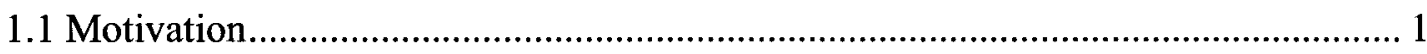

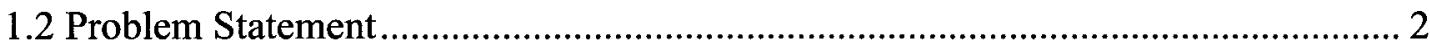

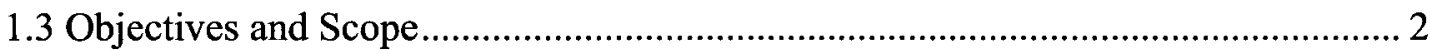

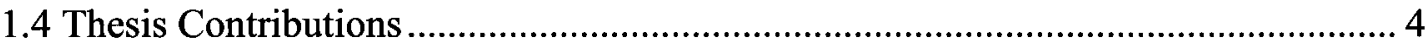

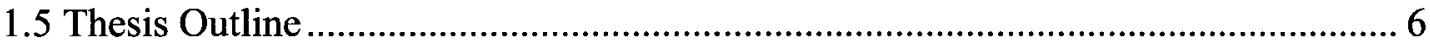

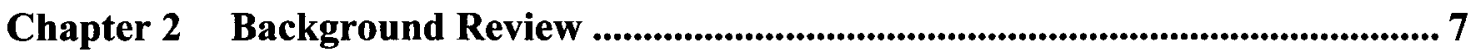

2.1 Domain of Remote Patient Monitoring ............................................................ 7

2.1.1 Introduction to Unobtrusive Smart Home Technologies ................................ 9

2.1.2 Current Bed Pressure Sensor Mat Technologies....................................... 10

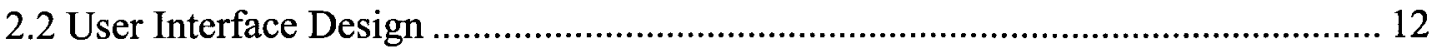

2.2.1 Introduction to the Human Factors Approach.......................................... 12 
2.2.2 Current User Interface Design Approaches for Home Care Monitoring .......... 16

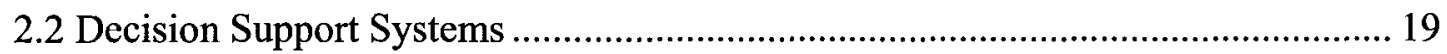

2.2.1 Introduction to Data Mining in Decision Support Systems ........................... 19

2.2.2 Current Clinical Trend Detection Techniques ........................................... 24

2.2.3 Introduction to Artificial Neural Networks............................................. 27

Chapter 3 Identifying User Needs and Establishing Requirements ....................... 31

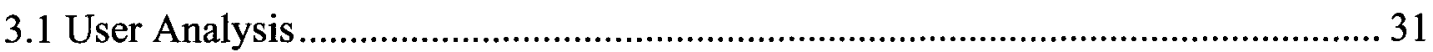

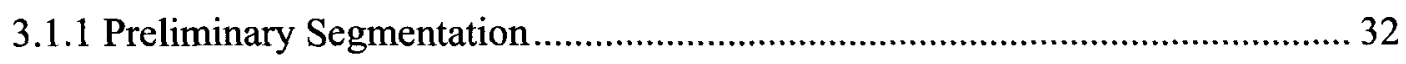

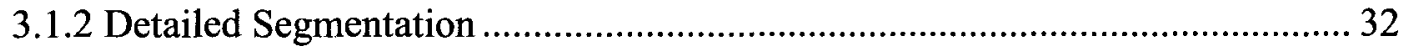

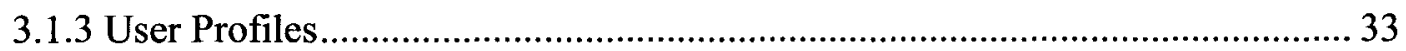

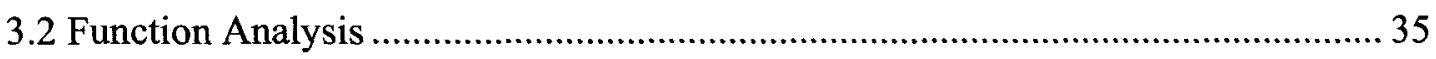

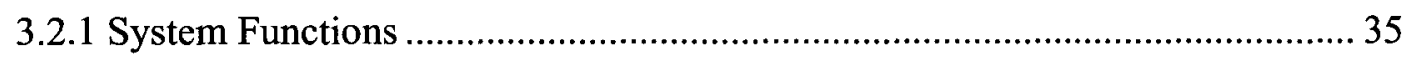

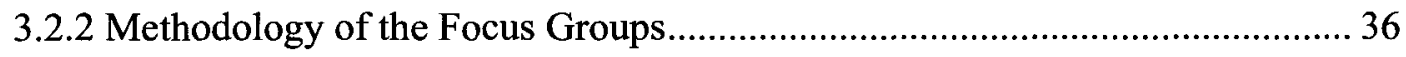

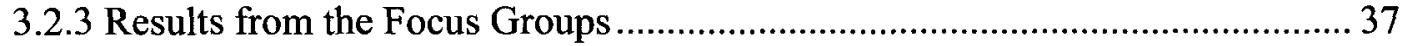

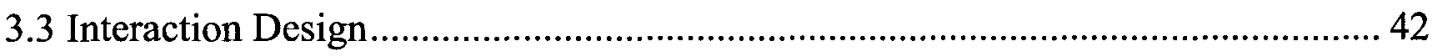

3.3.1 Usability and User Experience Goals ..................................................... 42

3.3.2 Design Process of and Rationale for the User Interface ............................... 43

Chapter 4 Graphical User Interface Usability Test .................................................... 53

4.1 Identification of Evaluation Objectives ..................................................... 53

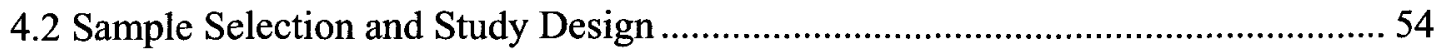

4.3 Selection of Representative Experimental Tasks and Contexts............................55

4.4 Selection of the Evaluation Environment …..................................................... 57 


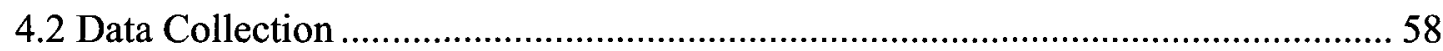

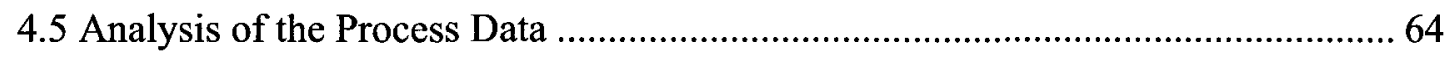

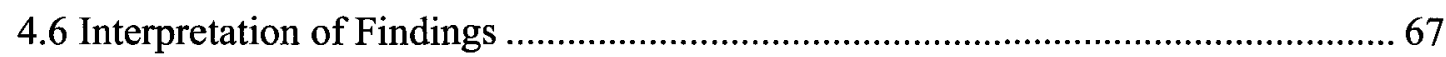

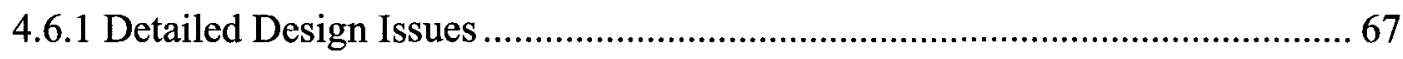

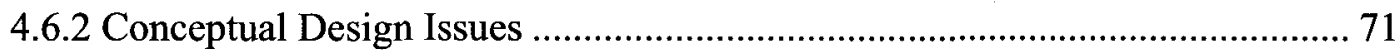

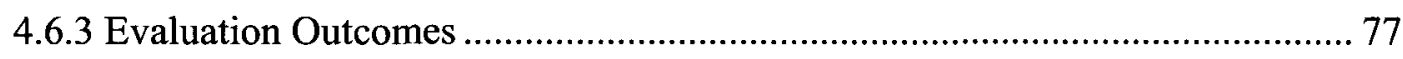

4.7 Iterative Input into Design ................................................................................. 78

Chapter 5 Data Mining Techniques for Home Care Client Health Data................. 80

5.1 Example Clinical Cases for Frequent Night-time Bed Exits ...................................... 80

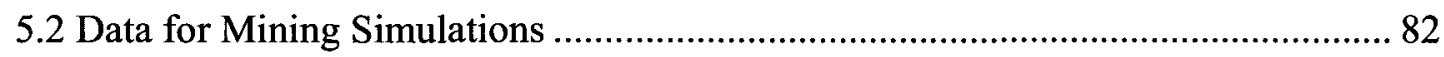

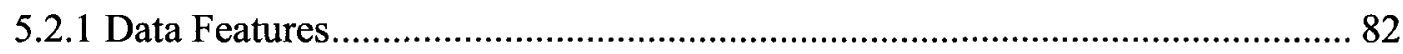

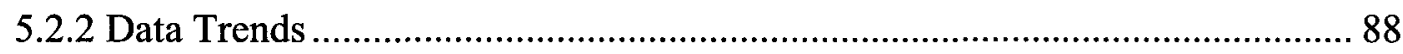

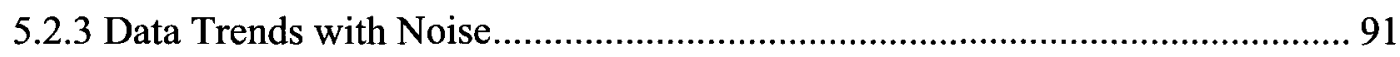

5.3 Detecting Trends Using a Segmentation Algorithm............................................. 93

5.3.1 Description of the Algorithm ........................................................................ 93

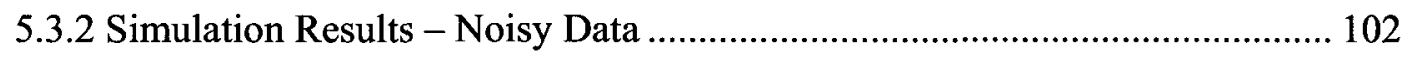

5.3.3 Simulation Results - Establishing Threshold Values ..................................... 113

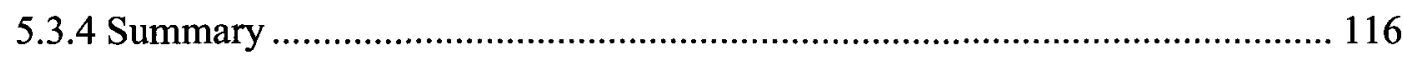

5.4 Detecting Trends Using a Neural Network........................................................... 117

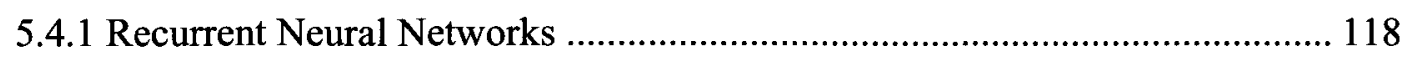

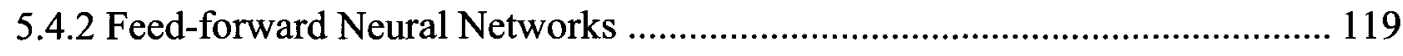

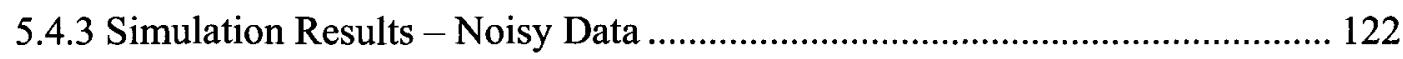


5.4.4 Simulation Results - Establishing Network Values

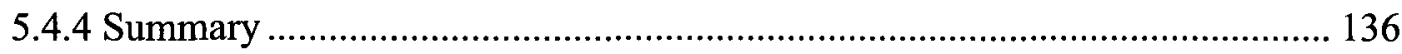

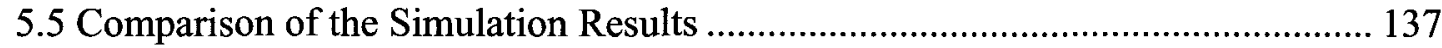

Chapter 6 Conclusions ..................................................................................... 143

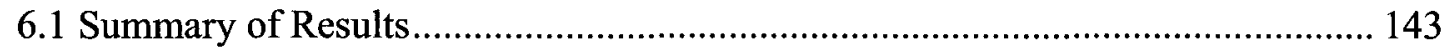

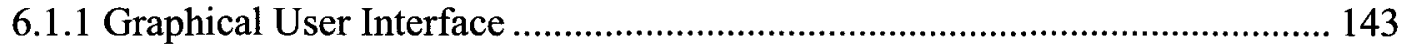

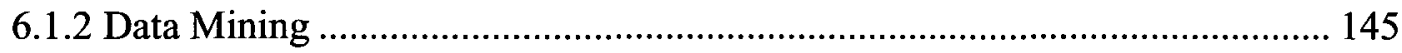

6.2 Recommendations for Further Research.................................................... 148

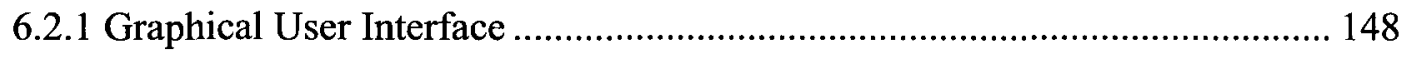

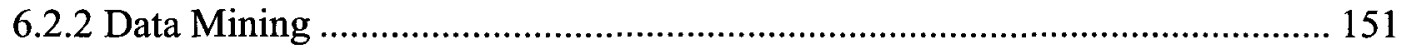

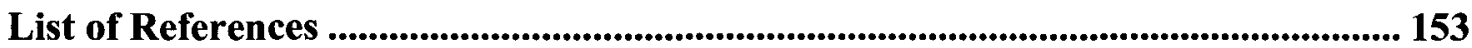

Appendix A: Other Medical Case Descriptions Used During the Usability Tests .. 165

Appendix B: Segmentation Algorithm - Optimal Parameter Plots .......................... 168

Appendix C: Segmentation Algorithm - Varying Noise Level Plots........................ 170

Appendix D: Feed-forward Neural Network - Optimal Parameter Plots ................ 180

Appendix E: Feed-forward Neural Network - Varying Noise Level Plots................ 182

Appendix F: Feed-forward Neural Network - Optimal Parameter Plots for Seven

Primitives................................................................................................................................ 192 


\section{List of Tables}

Table 2.1: Three tiers and clinical function tasks in health organizations [57]............... 22

Table 2.2: Commonly used neural network transfer functions [99] ............................. 29

Table 3.1: Detailed segmentation of primary and secondary users ............................. 32

Table 3.2: Key findings from the focus groups with home care clinicians...................... 39

Table 3.3: Usability and user experience goals of the graphical user interfaces.............. 43

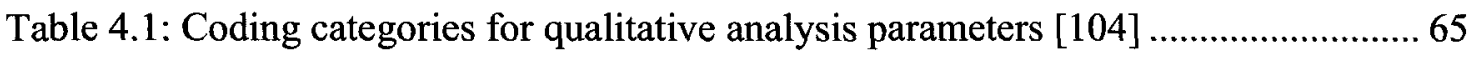

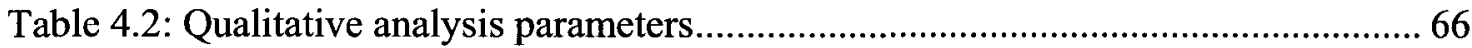

Table 4.3: Success of system as related to usability goals ...................................... 78

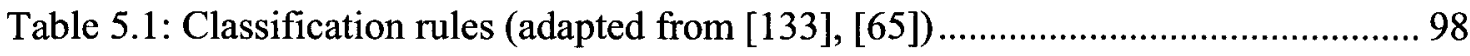

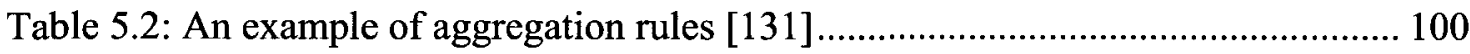

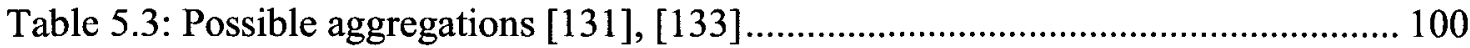

Table 5.4: Testing and final values of the tuning parameters for the simulated data sets 105

Table 5.5: Simulated data trend descriptions ...................................................... 106

Table 5.6: Testing and final values of the tuning parameters for the simulated data sets 124

Table 5.7: Noise levels (\%) and their corresponding magnitudes.............................. 140 


\section{List of Figures}

Figure 2.1 The cycle of human factors (adapted from [34]) ..................................... 13

Figure 2.2: An overview of the steps that compose of the knowledge-discovery in

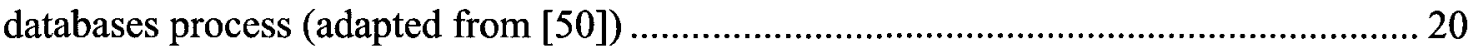

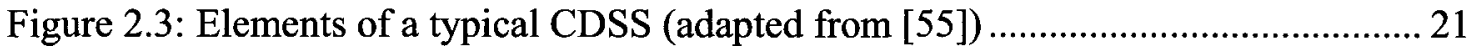

Figure 2.4: Schematic of supervised learning (adapted from [99]) ................................ 28

Figure 2.5: Schematic of a single hidden layer, feed-forward neural network (adapted

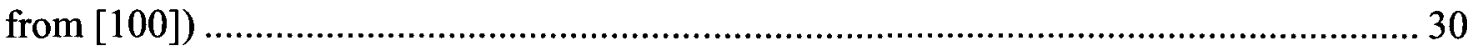

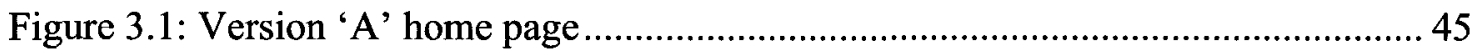

Figure 3.2: Version 'A' second level (L2) information reached from the button bar ....... 46

Figure 3.3: Version 'A' second level (L2) information reached from selecting a piece of

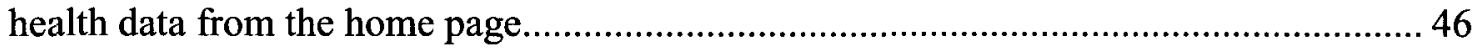

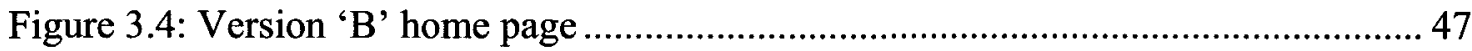

Figure 3.5: Version ' $B$ ' second level (L2) information reached from the button bar ....... 47

Figure 3.6: Version ' $\mathrm{B}$ ' second level (L2) information reached from selecting a piece of

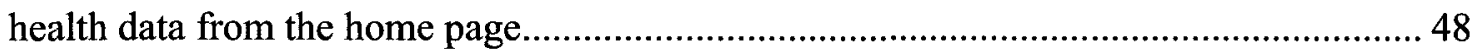

Figure 3.7: Representation of parameters on the homepage of Version 'A' (adapted from

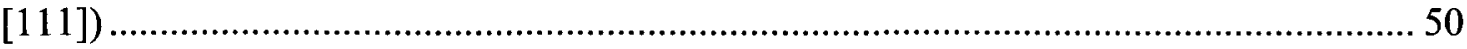

Figure 3.8: Example of a polar display for Version ' $\mathrm{B}$ ' ............................................... 51

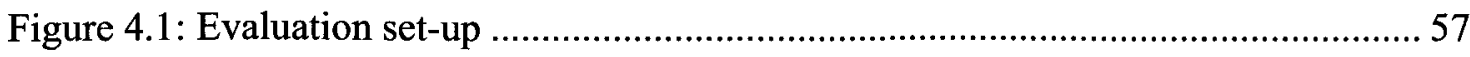


Figure 4.2: Video and audio based usability testing 63

Figure 4.3: Representation of detailed weekly information of parameters ........................ 68

Figure 4.4: Legend location and size for the homepage on Version ' $\mathrm{A}$ ' ........................... 69

Figure 4.5: Legend location and size for the homepage on Version ' $\mathrm{B}$ '.......................... 70

Figure 4.6: Bed activity tab in Version ' $\mathrm{A}$ ' .................................................................. 72

Figure 4.7: Bed activity tab in Version 'B' ................................................................. 73

Figure 4.8: Example of client target in L1 for Version 'B' '............................................ 74

Figure 4.9: Example of client target at L2 for A) Version ' $A$ ' and B) Version ' $B$ '.......... 74

Figure 4.10: Example of magnifying glass at $L 2$ for $A$ ) Version ' $A$ ' and B) Version ' $B$ ' 76

Figure 5.1: A simulated example of a home care client's bed exit data ............................ 83

Figure 5.2: Average and 'normal' minimum and maximum ranges of a home care client's

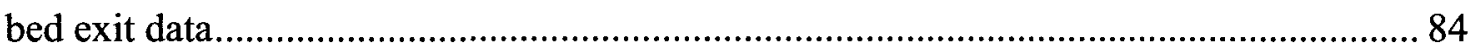

Figure 5.3: Least-square fit of a home care client's bed exit data .................................... 88

Figure 5.4: Primitives (adapted from [86]) ……………............................................. 89

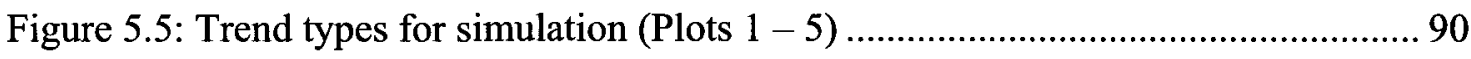

Figure 5.6: Plot 5 trend type with different levels of Gaussian noise .............................. 92

Figure 5.7: Example of a signal, extrapolation, and new model of the segmentation technique

Figure 5.8: Example of the CUSUM of the segmentation technique ……........................ 97

Figure 5.9: Classification features extracted from two consecutive segments (adapted

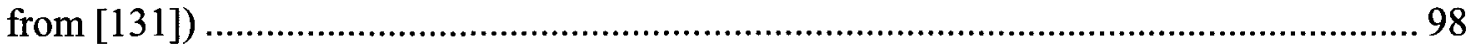

Figure 5.10: Simulated data, Plot 5, and the corresponding outputs from the segmentation

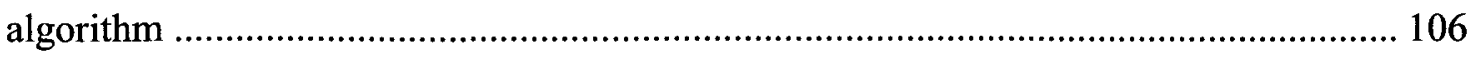


Figure 5.11: Plot 5 - 3\% noise 107

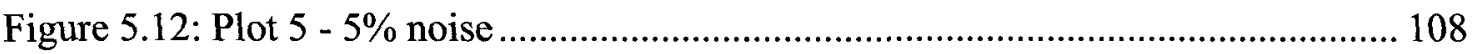

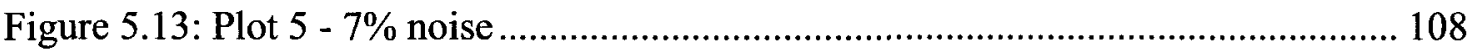

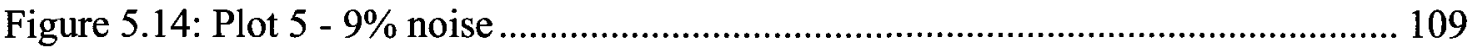

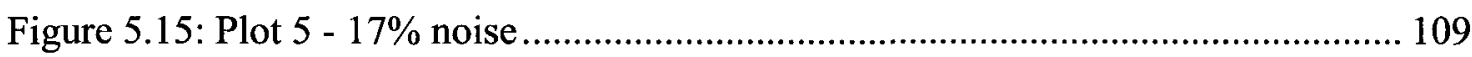

Figure 5.16: Plot 1 - Average performance at each noise level................................. 110

Figure 5.17: Plot 2 - Average performance at each noise level................................. 110

Figure 5.18: Plot 3 - Average performance at each noise level................................ 111

Figure 5.19: Plot 4 - Average performance at each noise level................................. 111

Figure 5.20: Plot 5 - Average performance at each noise level.................................. 112

Figure 5.21: Aggregate average results ........................................................... 113

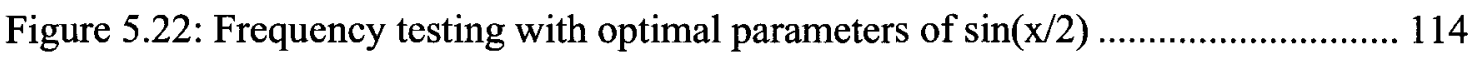

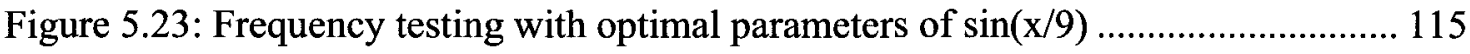

Figure 5.24: Frequency testing with optimal parameters of $\sin (\mathrm{x} / 2)$ and $\sin (\mathrm{x} / 9) \ldots \ldots \ldots 115$

Figure 5.25: Feed-forward neural network architecture ........................................ 120

Figure 5.26: Simulated data, Plot 5, and the corresponding output from the feed-forward

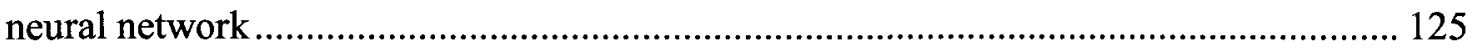

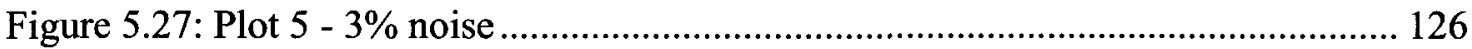

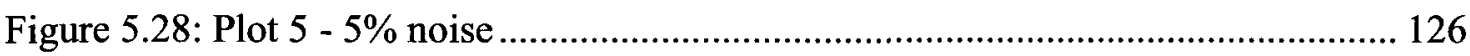

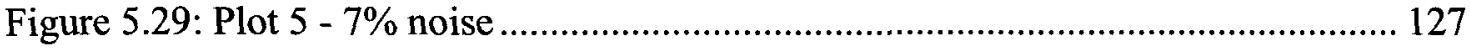

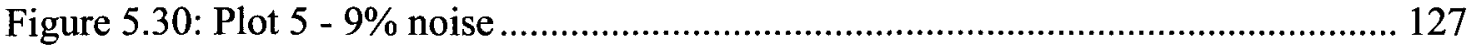

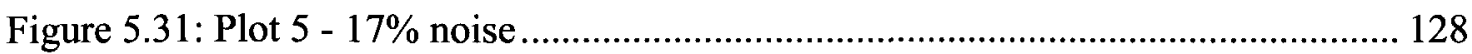

Figure 5.32: Plot 1 - Average performance at each noise level................................ 129 
Figure 5.33: Plot 2 - Average performance at each noise level................................... 129

Figure 5.34: Plot 3 - Average performance at each noise level................................ 129

Figure 5.35: Plot 4 - Averaged performance at each noise level................................. 130

Figure 5.36: Plot 5 - Average performance at each noise level.............................. 130

Figure 5.37: Combined average performance at each noise level for all plots .............. 131

Figure 5.38: Two scenarios demonstrating the advantages of the additional primitives 132

Figure 5.39: Simulated data, Plot 1, and the corresponding output from the feed-forward neural network using 7 primitives

Figure 5.40: Aggregated average results for feed-forward neural network using 3 and 7 primitives 134

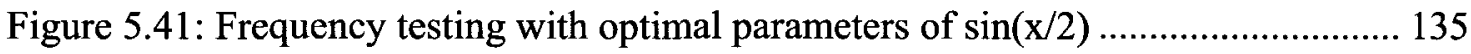

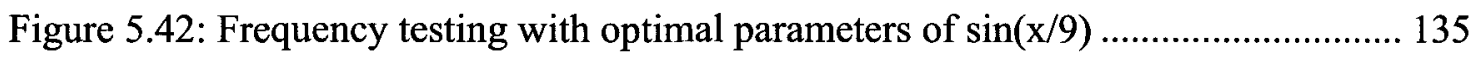

Figure 5.43: Frequency testing with optimal parameters of $\sin (x / 2)$ and $\sin (x / 9) \ldots \ldots \ldots 136$

Figure 5.44: RMSE for all noise levels and plots for both data mining techniques........ 138

Figure 5.45: \% difference for all noise levels and plots for both data mining techniques

Figure 5.46: Normalized RMSE for all noise levels and plots for both data mining techniques. 141

Figure 5.47: Aggregated average results for the segmentation algorithm, feed-forward neural network using 7 and 3 primitives 


\section{Chapter 1}

\section{Introduction}

\subsection{Motivation}

With increasing numbers of elderly putting pressure on health institutions, the need for new and innovative solutions for safe home health care has become imperative. For elderly clients who select this type of care, accurate techniques of monitoring and displaying their identified or developing health problems are crucial. Efficient and effective information transfer of changes in habits as well as abnormal events of the elderly client to their home care clinicians allows for an earlier response to a potential crisis and/ or preventive actions to be taken.

Home care clients can be monitored by unobtrusive sensors placed in their homes. These sensors can relay key information regarding the health of the client to home care clinicians for them to review and use as a decision support tool. The health information collected by these 'smart' sensors can be displayed on a graphical user interface (GUI) in order to supply current and continual information. Examples of smart sensors or smart monitoring technologies include: magnetic switches and motion, temperature, toilet flush, light and fridge door sensors, and pressure sensor mats, which can be placed is such areas as the bed, floor, bath, or chair. 
Displaying key health information on a GUI is an improvement over traditional paper charting as the data collected by the smart sensors allows for constant monitoring of home care clients. However, continual raw health data fed to clinicians is not useful. Data mining and an effective visual data display are required to meet the goal of highlighting useful information to support decision making by the clinicians. Specifically, there is a need to examine the appropriate visual data display features and to determine appropriate data mining techniques to provide data for the GUI. In addition, determining suitable data mining techniques will help prevent the subjectivity of visual inspection and provide simpler and relevant data trends for the clinicians.

\subsection{Problem Statement}

In the context of home health care, data mining techniques, which provide relevant data trends for decision support at point-of-care, have yet to be sufficiently addressed. "Pointof-care" refers to the location of being at or near the site of a client. Additionally, effective visualization techniques for clinical decision support systems are being pursued by a number of researchers but have not been substantially evaluated in home care.

This thesis focuses on home care clinician's user requirements for data visualization and data mining techniques that are most suitable for monitoring home care clients to produce relevant data trends.

Furthermore, this thesis analyzes the data mining techniques for their accuracy in detecting trends when various noise levels are applied to different data trends types and suggests means of establishing the parameter values needed for the techniques.

\subsection{Objectives and Scope}

The following statements outline the scope of this thesis: 
- This thesis focused on utilizing bed pressure sensor mat technology as an example for data visualization and data mining techniques and also extrapolates to more general applications.

- The target primary user groups were guided by the organizations listed below and include: home care nurses, physiotherapists, occupational therapists, and case managers. There are other primary user groups involved in home care but for the purposes and scope of this work, were not involved.

- The types of technologies considered for the interface and usability testing were guided by the current research of Wilfrid Laurier University and Carleton University. Specifically, the technologies include Wilfrid Laurier University's GPS-supporting monitoring system to track clients' daily activities and health conditions and Carleton University's unobtrusive smart home technology sensors, such as a bed pressure sensitive mat.

The following objectives have been identified:

- Examine and compare machine learning techniques, a segmentation algorithm and a neural network, for trend detection;

- Determine a means for establishing the values of the segmentation algorithm's and neural network's parameters;

- Determine home care clinicians' preferences and expectations in respect of a decision support system's user interfaces;

- Propose visualization and interaction methods of a decision support system's graphical user interfaces displaying health information for smart home clients; and

- Evaluate prototype interfaces of the decision-support system. 
The team involved in various components of the research in this dissertation was a multidisciplinary team drawing from systems and computer engineering, biomedical engineering, psychology, geography, and nursing. Specifically, the institutions that are a part of some of this research include: the Faculty of Engineering and Design at Carleton University, the Lawrence S. Bloomberg Faculty of Nursing at the University of Toronto, and the Department of Geography and Environmental Studies at Wilfrid Laurier University. The organizations involved include: Saint Elizabeth Health Care, SCO Health Services, Nortel, HInext and Research in Motion. The members from these institutions and organizations are referred to as the 'research group' in parts of Chapter 2 and 3.

The techniques presented herein for analysis of home care client data are technology independent. Experimental data was generated and modeled after data that can be obtained from a Kinotex pressure sensors mat, generously donated by Tactex Controls Inc.

\subsection{Thesis Contributions}

The following is a list of contributions that were made during research for this thesis.

- A qualitative and quantitative comparison between a segmentation algorithm and a feed-forward neural network for trend detection using noisy data was conducted.

- In order to produce optimal results for a variety of data trend types, a process for establishing values of the segmentation algorithm's and neural network's parameters is proposed.

- To identify user needs and establish requirements for a decision support system, a variety of home care primary end user groups participated in focus groups. A conference presentation detailing the methods and results was accepted and 
presented at the 2009 Canadian Nursing Informatics Association in Mississauga, Ontario, November 2009 [1]. Additional results regarding concept development and prototype testing were accepted and presented as podium presentations at the $23^{\text {rd }}$ Annual Research Conference in London, Ontario, April 2010 [2] and the $4^{\text {th }}$ National Community Health Nurses Conference in Toronto, Ontario, June 2010 $[3]$.

- Graphical user interface usability tests with the primary end user groups were conducted as related to integrated data gathered from a bed mat pressure sensor mat and GPS-supporting monitoring system. A poster presentation regarding the method, qualitative analysis, and identified data visualization techniques that are suitable for monitoring home care clients was accepted and presented at the eHealth 2010 conference in Vancouver, British Columbia, June 2010 [4].

- Additional data visualization techniques extrapolated to a GPS-monitoring system were established. A poster presentation displaying the suggestions and possible system architecture for an automated monitoring system was accepted to the eHealth 2010 conference in Vancouver, British Columbia, June 2010 [5].

- The development process of a prototype web-based platform for presenting biomobility data to health care workers and integrating it with best practice guidelines in order to facilitate evidenced-based practice at the point-of-care was proposed. A poster presentation demonstrating the concept and methodology was accepted to the e-Health 2010 conference in Vancouver, British Columbia, June $2010[6]$. 


\subsection{Thesis Outline}

Chapter 2 provides a literature review of remote patient monitoring, user interface design, and decision support systems. It specifically introduces the pressure sensor mat that will be used in examples throughout the dissertation, user interface design approaches for remote monitoring, and the trend detection techniques used in later chapters.

Chapter 3 identifies home care clinicians needs and establishes requirements for a decision support system. Specifically, the chapter provides user and function analyses, outlines the usability goals, and describes the design process and rationale of the interface of the system.

Chapter 4 discusses the process of preparing for a usability test of the two graphical user interface prototypes designed in the previous chapter. It also identifies the evaluation objectives, describes the methods of analyzing the results, and presents the findings of the test.

Chapter 5 describes example clinical cases in which features of a home care client's data must be detected. It introduces specific trends that exist in data and presents two different types of data mining techniques: a segmentation algorithm and a feedforward neural network. Each of these techniques are analyzed for their accuracy in detecting underlying trends in noisy data and a process is introduced for establishing values of their parameters.

Chapter 6 presents the thesis conclusions which include a summary of the results and a list of recommendations for future work.

References and appendices containing additional use cases from Chapter 4 and results from Chapter 5 are located in Appendices $A-F$. 


\section{Chapter 2}

\section{Background Review}

This chapter presents background information regarding the domain of remote patient monitoring, remote patient monitoring technologies, pertinent literature regarding human factors and user interface design, and data mining techniques used in clinical decision support systems.

\subsection{Domain of Remote Patient Monitoring}

There are two notable trends in Canada's demographics. The first is that life expectancy has increased steadily during the twentieth century and the second is the proportion of elderly people has grown [7]. Both of these compelling trends must be addressed in health care planning as it is essential that older adults who want to live independently in their homes as they age can be effectively supported by the health care community. In $2009 / 10$, approximately 603,000 people in Ontario received home care funded by the Ministry of Health and Long Term-Care [8]. It has also been shown that the majority, about two-thirds [9], of individuals currently receiving home care support, are elderly and live alone [10].

For the elderly, independent lifestyle comes with risks. These risks can be additionally complicated by chronic illness and immobility, cognitive and sensory 
impairments [11]. Other risks arise from the intermittent care of the elderly at home as health care providers are only able to visit each client's home for a short time. On average, each visit is about 45 minutes long and visits only occur a few times a week [12].

Replacing a clinical environment with a home environment could greatly reduce the increasing pressure on health care providers. However, in this case, there becomes a need for adequate remote data collection for the health care provider. A great amount of medical data can be collected remotely by low-cost sensors. Once the large datasets collected by the sensors are correctly processed to obtain relevant data and presented in a manner that is useful and clear, the data can provide an ongoing history of the home care client to the appropriate clinician. This type of real-time monitoring would also offer care providers alerts to problems of the client's current condition and allow the client to remain in the comfort and familiarity of their own home [13].

Homes installed with various digitally controlled, interconnected systems are referred to as 'smart homes' [14]. The smart residence is equipped with technology that improves the safety of home care clients and monitors the condition of their health. As such, any chosen devices or sensors installed into the residence should address the clients' functional limitations and their social and health care needs [11]. In the case of this research, the chosen device will be discussed in sub-section 2.1.2.

There are a number of academic institutions and organizations that are currently researching new approaches to monitoring clients remotely. An example is Continua Health Alliance. The Alliance is an organization that consists of multiple company collaborations in technology, medical device, and health and fitness. Some companies within the Alliance include IBM, iMetrikus, Intel, Medtronic, Philips and Sprint. A few 
examples of the personal remote monitoring devices and services explored by the Alliance include blood glucose tests, blood pressure monitors, pulse oximeters, other basic vital sign monitors, motion sensors, medication reminders, and emergency response services [15].

\subsubsection{Introduction to Unobtrusive Smart Home Technologies}

There are many unobtrusive technologies that can support remote patient monitoring and most cannot only be implemented in the home but also in other environments such as hospitals and nursing homes.

Ambient sensors tested in an eldercare facility by Demiris et al. [16] included bed pressure sensors (which will be discussed in detail in the following section), gait sensors (pressure mats), video cameras and stove sensors.

As detailed by Scanaill et al. [17], sensors employed in health smart homes can include pressure sensors, pressure mats, smart tiles, passive infrared sensors, sound sensors, magnetic switches, active infrared sensors, and optical/ultrasonic systems.

Virone et al. [18] describes a medical sensor network system with some wearable sensors and some placed inside the living space. A variety of sensors and devices are used in the architecture such as: activity sensors, physiological sensors, environmental sensor, pressure sensor, RFID tags, pollutions sensors, and a floor sensor.

A review of assistive technology in elderly care by Miskelly [19] outlines a number a recent developments in new technology. The technologies include: community alarms, video monitoring, health monitors worn on the wrist, fall detectors worn around the waist, hip protectors, pressure mats, door alerts, infra-red movement detectors, dawn/ 
dusk lights, smokes and fire alarms, cooker controls, electronic calendars, and speaking clocks.

Carleton University is a partner in a smart home project called Technology Assisted Friendly Environment for the Third Age (TAFETA) which is located at the Élisabeth Bruyère Hospital in Ottawa, Canada. The prototype apartment is currently equipped with a number of smart home sensors and sensors undergoing experimentation in the Digital Signal Processing lab at Carleton University. As detailed by Arcelus et al. [20], these sensors include magnetic switches, thermistors, accelerometers, radio frequency identifications, infrared motions sensors, microphone arrays, smart grab bars, pressure sensitive mats, and electronic noses.

\subsubsection{Current Bed Pressure Sensor Mat Technologies}

As introduced above, there are a number of emerging technologies used for non-invasive monitoring of human activity during sleep. The use of load cells or force sensors for this type of monitoring is the most common approach and have been employed by several researchers [21], [22] some of which are outlined below.

Virone et al. [18] outlines one such pressure sensor that uses an air bladder strip located on the bed in order to measure the breathing rate, heart rate and agitation of a patient. Also measuring similar parameters is a SleepSmart device by Loos et al. [23] which is a multi-sensor mattress pad controlled by software to detect heart rate, breathing rate, body orientation and index of restlessness. The mattress pad is composed of 54 force sensitive resistors and 54 resistive temperature devices. Another force sensor is a robotic bed presented by Nishida et al. [24]. It is equipped with 221 pressure sensors has been used to monitoring respiration and body position. 
There have also been many pad-based solutions proposed [25]. The static charge sensitive bed has been used for monitoring motor activity by several authors [26]-[28] The bed is composed of "two metal plates with a wooden plate in the middle that must be placed under a special foam plastic mattress, which operates like a capacitor" [25].

TigerPlace, an independent senior housing that helps residents age in place, currently uses bed sensors to monitor the residents. The bed sensor is a padded pneumatic strip that lies on top of the bed mattress and under the linens. It is used to detect presence in bed and measure restlessness, qualitative breathing and pulse as a person sleeps [29].

Another sensing technique for body movement in bed is optical fibres and conductive fibres. A body movement monitoring system using optical fibres was proposed by Tamura et al. [30] and Spillman et al. [31] and one which uses conductive fibre sensors to detect body position, respiration, and heart rate was designed by Kimura et al. [32].

For the purpose of this research, the type of sensors used are those composed of pressure sensor arrays which can measure the force applied at a number of sensing points. The bed occupancy sensor from Tactex Controls Inc. has 24 pressure sensor in a $3 \times 8$ grid configuration and occupies $24 \mathrm{~cm} \times 90 \mathrm{~cm}$ with sensor elements spaced $10 \mathrm{~cm}$ apart. The data collected from these sensors allow for the creation of pressure images and videos and is placed between the top and bottom mattresses [13]. 


\subsection{User Interface Design}

\subsubsection{Introduction to the Human Factors Approach}

As adopted by the International Ergonomics Association [33], the definition of the field of human factors is

the scientific discipline concerned with the understanding of interactions among humans and other elements of a system, and the profession that applies theory, principles, data, and other methods to design in order to optimize human well-being and overall system performance.

Applying a human factors approach to the design process of a decision support system has three aims: to assure that the proposed system enhances the current practices of monitoring clients in their homes, to increase safety of the clients, and to increase both homecare clinicians' and clients' satisfaction [34]. A decision support system is a computer application designed to support decision making activities. It has also been shown that presenting patient-specific recommendations through the use of a clinical decision support system, in a way that can save clinician time, produces highly effective, sustainable tools for altering the behaviour of clinicians [35]. More details regarding this type of decision support system can be found in section 2.2.

There are several approaches in the human factors cycle that help to satisfy the three goals listed above. The human factors cycle can be represented by the human operator (brain and body), the system that the individual is interacting with, and the

research team (see Figure 2.1). If a human factors solution is required because of a problem or deficiency (e.g. accident or incident) in the human-system interaction, the 
cycle begins at Point $\mathrm{A}$. If adequate human factors are desired at the beginning of a design cycle, the cycle begins at Point B.

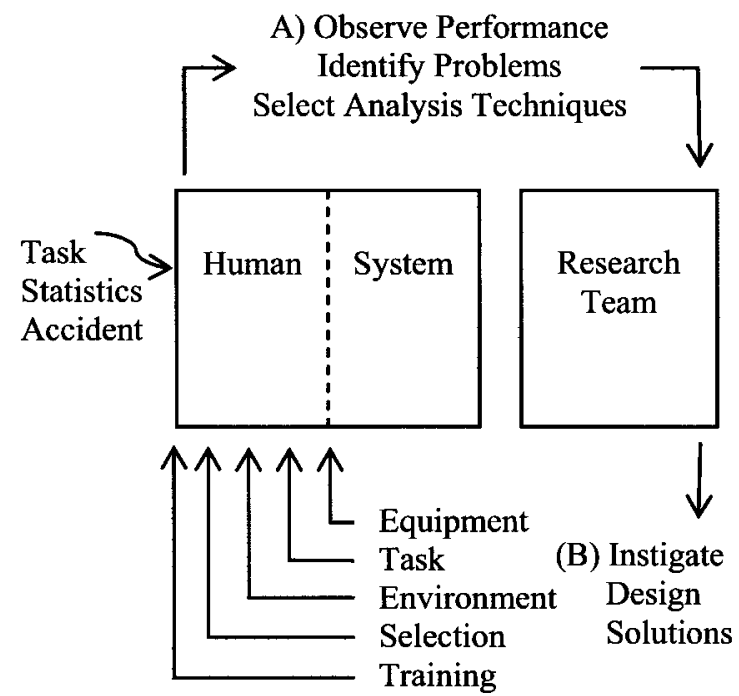

Figure 2.1 The cycle of human factors (adapted from [34])

The first step in the cycle is to identify problems and deficiencies in a humansystem interaction of a current system. The entry point of this cycle is at Point (A) in Figure 2.1. Once these have been identified, there are five different approaches which may be taken in order to implement a solution. These approaches include: equipment design, task design, environmental design, training, and selection. Entering the cycle at Point (A) of the cycle is for fixing existing problems.

Entering the cycle at the second step (i.e. Point (B) in Figure 2.1) and designing systems which are effective from the initial stages of product design, is just as relevant as the first method. Entering the cycle at this point helps to prevent human factors deficiencies before they affect the system design [34].

The organization from which clinicians were recruited for this research currently supports independent living elderly clients through intermittent home visits by a home 
care clinician. During these visits, the home care clinician assesses a client by charting observations on paper. A problem associated with this system includes the feeling of isolation from both clients and health care providers because the visit is only one 'snapshot' of the client and may not give a good overall continual assessment of health. This may be attributed to the challenges of health service delivery and Canada's increasing home care expenditures due to a changing demographic [36].

From a client's perspective, a remote monitoring system may ease the feeling of isolation due to the ability to monitor their mobile and cognitive function continuously, provided a clinician checks in with them every so often. Therefore, cost effective solutions need to be implemented in order to manage both the increasing costs and the social challenges of providing health care to an aging population who want to live at home independently.

Given that this research is proposing to design a new system, namely one that will monitor these clients remotely by means of novel smart home technologies, the entry point of the cycle is at Point (B) in Figure 2.1.

The methodological principle of the field of human factors, which encompasses all the methods and techniques discussed above, is to center the design process around the user. This process is termed "user-centered design." In order to assure that research follows this principle, user needs must be determined sufficiently and the users must be involved at all stages of the design process [37].

For this research, the home care clinicians task performance must be examined, their needs, preferences, insights and design ideas obtained, and their response to design 
solutions requested. Executing these steps assures that the design satisfies the user's needs rather than forcing the user to adapt to a system [34].

The other rationale for developing an understanding of real-life point-of-care scenarios is to assure that the system addresses appropriate "use cases". A use case is a situation where the system could aid in facilitating a patient care decision. Determining information flow drawn from the scenarios will also address use cases [34]. The smart monitoring technologies could be used to assist in diagnosing a health concern, educate clients, monitor mobility, determine compliance with treatment directives, promote client self-care, or to monitor recovery.

Evaluating the usability and safety of the system in a simulation laboratory setting is also an important step in ensuring a successful solution. A laboratory setting will help minimize exposing home care clients or clinicians to potential risks in a live home environment.

A user-centered design process is the primary problem solving method of a usability engineer. Four common approaches of usability engineering for software design have been outlined by Neilson [38]. These include the following:

- Early focus on the user and tasks,

- Empirical measurement using questionnaires, usability studies, and usage studies focusing on quantitative performance data,

- Iterative design using prototypes, where rapid changes are made to the interface design, and;

- Participatory design, where users are directly involved as part of the design team. 
All four approaches will be followed in order to determine the final solution of this research. Details of these steps can be found in Chapter 3 and 4.

\subsubsection{Current User Interface Design Approaches for Home Care Monitoring}

Electronic health records (EHRs) which contain clinical decision support systems as well as a plethora of electronic health information, have had slower adoption rates than expected. Aside from initial cost and lost productivity during implementation, there exists a lack of efficiency and usability in the design of EHRs. Usability can be defined as "the effectiveness, efficiency, and satisfaction with which specific users can achieve a specific set of tasks in a particular environment" [39]. Similar deficiencies exist in interface designs of such systems in home health care.

Performing a usability evaluation includes measuring user satisfaction, efficiency, effectiveness, cognitive load and ease of learning. "Usability emerges from understanding the needs of the users, using established methods of iterative design and performing appropriate user testing when needed" [39]. There exists a wide variety of subjective and objective design and evaluation methodologies and evaluation settings. For example, researchers can "test" in live clinical settings by built-in webcams on modern laptop PCs, robust wireless networking, remote testing software, and compact inexpensive video recorders [39].

Developing an EHR with excellent usability is a challenge because there is a wide range of complex information needs which differ from setting to setting, between user groups, and from task to task. Other factors include the mobility of clinicians, their primary focus devoted to a patient, multi-tasking, interruptions, and frequent agenda 
changes during a single patient workflow [39]. Other challenges in developing EHRs are also discussed in sub-section 2.2.1.

Considering the complexity of implementing EHRs, very little research has been conducted regarding remote monitoring systems for home care and involving clinicians in some facet of their design [40]. There are also a number of commercial data mining packages available to help display time-oriented data and information regarding patients [41], but again their development has not always included end-users. It has also been demonstrated that improvements of overview and navigation of EHRs can be accomplished by using graphical timelines. The following examples provide an overview of several projects which have involved home care clinicians in some aspect of interface design for time-oriented data and some which have not.

Example 1. Researchers at the University of Missouri have been developing an EHR for TigerPlace, an independent senior housing that helps residents age in place, with the guidance of clinicians. TigerPlace is equipped with an unobtrusive integrated monitoring system for acquiring data from elder residents and their environment and displays this information on their EHR. The research involved the end users, nurses, in the design of the interface for the display of the monitoring data related to the residents' activity levels and sleep patterns. The web-based interface shows histograms of bed restlessness and pulse aggregated to a daily level [16], [29].

Example 2. A smart home environment which includes an accelerometer-based mobility tele-monitoring device is described by Scanaill et al. [17]. The accelerometer classifies activities such as: lying, sitting, standing and walking. This information is relayed to a server via SMS messages. This study conducted a testing phase in which 
usability and effectiveness of the design was examined before deployment into elderly people's homes.

Example 3. Scandurra et al. [40] used participatory design to develop and deploy a mobile Virtual Health Record on a PDA with experienced homecare staff. The staff involved included general practitioners, district nurses, and home help service personnel, mainly assistant nurses.

Example 4. A usability test with six diabetes care providers was conducted on a Comprehensive Diabetes Management Program by [42]. The clinicians were observed while performing standardized tasks with the application and then completed a usability questionnaire and interviews. The study confirmed that there is a need for usability testing of health application, even those developed by experts.

Example 5. Casper et al. [43] describes the process used to design the HeartCare website which supports technology enhanced practice for home care nurses whom provide care for patient with congestive heart failure. The website includes functions such as communication, information and self-monitoring. A design work system analysis and focus groups utilizing participatory design methodology were undertaken. Future steps of the project include validation and refinement of the website and planning for user training activities through a two-stage usability test.

Example 6. Virone et al. [18] describe the design of four different graphical user interfaces for an assisted living oriented information system. One of the interfaces is designed for a local nurse control station and another on a PDA for health care providers. It was not identified in this study whether or not clinicians were involved in part of the design. 
Example 7. A study by Jung et al. [44] describes the process and methodology of designing and developing a clinical decision support system for home health care system. The system acquires eight health parameters, monitors the information, and classifies it according to identified health levels. Within the design and development stages of the system, there was no indication of clinical user involvement.

Example 8. An interface was designed in [45] for sleep activity pattern monitoring application but it unknown whether clinicians were used in its conception and if usability testing was executed.

\subsection{Decision Support Systems}

\subsubsection{Introduction to Data Mining in Decision Support Systems}

Data mining is the process of discovering patterns from data sets. It typically involves transferring data collected from a system into a database, cleaning the data to rid of errors, checking consistency of formats, and then examining the data using statistical queries, neural networks, or other machine learning techniques [46]. A detailed explanation of neural networks is presented in the sub-section 2.2.3.

Areas in which pattern recognition is of great importance are machine vision, character (letter or number) recognition, computer-aided diagnosis, and speech recognition [47]. The work presented here focuses on computer-aided diagnosis.

The following are examples of data mining methods:

- Classification is learning a function that maps (classifies) a data item into one of several predefined classes [48], [49]. Some example algorithms include: decision tree learning, nearest neighbour, naïve Bayesian classification, and neural networks. 
- Regression is learning a function that maps a data item to a real-valued prediction variable [50].

- Clustering is a common descriptive task where one seeks to identify a finite set of categories or clusters to describe the data [51], [52].

- Association rule learning is a method for discovering interesting relations between varıables in large databases [53].

Data mining is a step within the knowledge-discovery in databases (KDD) process (Figure 2.2). At the end of the process, acting on the discovered knowledge takes place. This includes: using the knowledge directly, incorporating the knowledge into another system for further action, or simply documenting it and reporting it to interested parties [50]. This final step involves communicating the knowledge discovered to a party such as home care clinicians to aid in decision making. Communication portals that the KDD process supports are clinical decision support systems. These systems belong to a large family of decision tools that have been dubbed as "active knowledge" [54] systems that use patient data to generate case specific advice to support decision making.

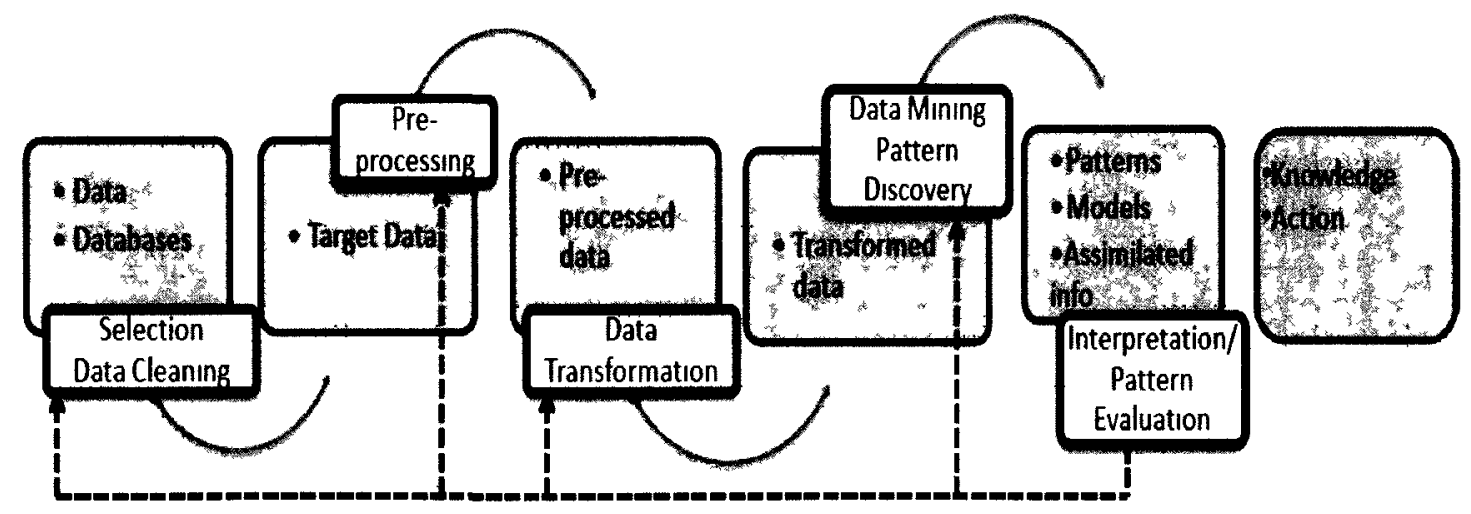

Figure 2.2: An overview of the steps that compose of the knowledge-discovery in databases process (adapted from [50]) 
Clinical decision support systems (CDSS) are "computer applications designed to aid clinicians in making diagnostic and therapeutic decisions in patient care" [35]. There are a number of advantages for their use, some of which include: simplifying access to data needed to make decisions, providing reminders and prompts at the time of a patient encounter, assisting in establishing a diagnosis and in entering appropriate orders, and alerting clinicians when new patterns in patient data are recognized [35].

A CDSS typically has four basic components: inference engine (IE), knowledge base (KB), explanation module, and working memory (Figure 2.3). The IE uses the knowledge of the system and that regarding the patient to generate conclusions about certain conditions and controls the actions which need to be taken by the system. The IE obtains its knowledge from the KB. The $\mathrm{KB}$ can be built from the experience of a domain expert or by an automated process such as a computer application that gathers its knowledge from databases, books, and journal articles. The explanation module creates and displays the rationalization for the conclusions generated by the IE [55].

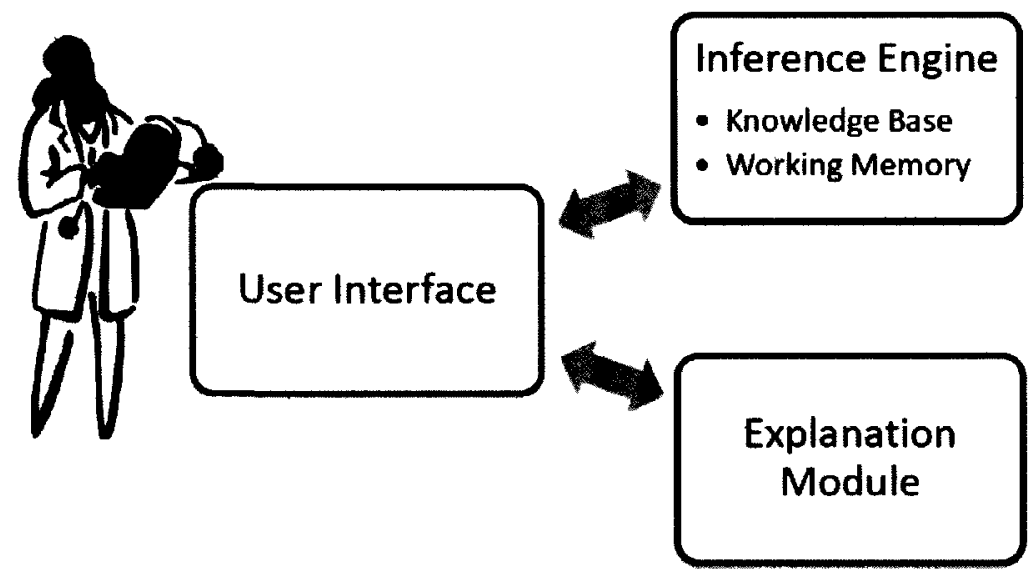

Figure 2.3: Elements of a typical CDSS (adapted from [55])

Another type of CDSS is non-knowledge-based and uses a form of artificial intelligence called machine learning. This type of CDSS allows computer programs to 
learn from past experiences. A program learns when its performance at certain tasks improves with experience. In practice, machine learning algorithms are of special use in data mining scenarios involving large databases. Examples of machine learning techniques are decision trees and forests, artificial neural networks and Bayesian networks [56].

Table 2.1 illustrates the three tiers and clinical function tasks in health organizations. Similar types of tasks using CDSS can be extrapolated into the domain of home health care and will be further discussed in sections 3.1 and 3.2.

Table 2.1: Three tiers and clinical function tasks in health organizations [57]

\begin{tabular}{|c|c|c|}
\hline \multicolumn{2}{|c|}{ Health Organization Level } & \multirow[b]{2}{*}{\begin{tabular}{l}
\multicolumn{1}{c}{ Tasks } \\
Diagnosis \\
Causal analysis \\
Clinical reasoning \\
Recommendation \\
Interpretation \\
Prediction
\end{tabular}} \\
\hline Top & $\begin{array}{l}\text { Physicians } \\
\text { Surgeons } \\
\text { Medical directors } \\
\text { Residents } \\
\text { Specialists }\end{array}$ & \\
\hline Middle & $\begin{array}{l}\text { Physician assistants } \\
\text { Nurse practitioners } \\
\text { Nurses }\end{array}$ & $\begin{array}{l}\text { Alerting } \\
\text { Monitoring } \\
\text { Clinical reasoning } \\
\text { Clinical function } \\
\text { Reminding }\end{array}$ \\
\hline Lower & $\begin{array}{l}\text { Nurses } \\
\text { Clinical assistants } \\
\text { Discharge planners } \\
\end{array}$ & $\begin{array}{l}\text { Alerting } \\
\text { Reminder }\end{array}$ \\
\hline
\end{tabular}

For clinicians, it is important to observe and monitor certain features from patient data in order to aid in decision making and reduce cognitive overload. Visually communicating temporal pieces of information, also known as temporal episodes, to clinicians can provide a means of understanding the evolution in time of a feature and aid in quicker decision making. Extracting patterns in data can also reduce cognitive load. Any patient, whether under the surveillance of home care sensors or in an intensive care 
unit, may have a number of monitoring systems recording physiological parameters. Due to technological advances, many of these parameters are recorded at high frequency rates and the amount of data they provide is quite overwhelming. In addition, a clinician could also be providing care for a number of patients having diverse medical conditions [58].

When monitoring a patient's health data, it is important to observe and record clinical events and detect and potentially prevent adverse events. One feature in a patient's health data which may indicate important events is the characteristic of a trend. Once this feature has been detected from a patient's data, an alarm can be sent to a clinician to warn them of a transient variation which may be of clinical significance. Details of mining this feature from a patient's data are presented in Chapter 5.

Implementing a CDSS is a challenging process and requires an intelligent computing infrastructure in which patient data is in a machine-processible form and changes be made to existing workflow [35]. Another barrier for the sustainability of CDSS is the significant cost of knowledge acquisition and knowledge maintenance. There are many other concerns as well, such as the adoption of standards of interoperability, proper validation of the programs, and concerns that health care professionals are "not well prepared to meet society's expectations with regard to evidence-based practice and the use of information technology in the delivery of health care" [55]. Despite some difficulties in creating and implementing a CDSS, it has been shown in a wide range of clinical settings that these systems help provide better quality of care of patients by clinicians [35]. 


\subsubsection{Current Clinical Trend Detection Techniques}

Patient data collected for monitoring purposes is usually of two types: acute monitoring in an intensive care unit or discrete monitoring over long periods of time (e.g. patients with chronic diseases). Both types involve the measurements of parameters at different times and therefore, the temporal component needs to be taken into account for data analysis and in the design of analysis tools for prediction, intelligent alarming and therapy support [59]. Many different methods have been proposed and investigated for trend detection, mostly from the fields of statistics and artificial intelligence [60].

\section{Statistical Approaches}

Robust regression techniques can be implemented to extract an underlying signal from noisy physiological time series, e.g. the repeated median [61], in a moving time window [62]. The results of using such a technique can be further improved by integrating online outlier replacement rules [63] and by reducing the time delay of the estimation [64].

Charbonnier and Gentil [65] discuss recent developments of a trend-based alarm system introduced in Charbonnier et al. [66] to improve patient monitoring in intensive care units. The system utilizes a segmentation algorithm, a classification of the segments into seven temporal shapes, and a temporal aggregation of episodes. The algorithm is capable of detecting trends through splitting the data into linear segments of different lengths by combining linear least squares regression for data approximation. To verify if the current approximation is still acceptable, the cumulative sum technique is employed. The quantitative information is used for generating alarms and the shape information is used for detecting specific clinical situations. 
Mäkivirta et al. [67] demonstrated the ability of two median filters, with different window lengths, to monitor intensive care time series. The filters eliminated noise and particularly artefacts before the actual analysis of monitoring data.

Conventional control charts have also been used to detect 'out-of-control' states in a process [68] and [69] but have been deemed problematic because they are based on a fixed target value. Often for medical application, there is "not a unique target value for different individuals or health states" [60]. Additionally, this method does not account for autocorrelation and does not distinguish between different patterns of change, e.g. outliers, level shifts and trends.

Smith et al. [70] applied time series methods to online monitoring data for patients after kidney transplantation by using a multiprocess Kalman filter to identify types of change and online-probabilities for the occurrence of change. The approach was validated by Trimble et al. [71] and modifications presented in [72] and [73]. In addition, Yang et al. [74] improved on the technique by also employing exponentially weighted moving average prediction and cumulative sum testing to identify change-point in heartrate time series [60]. Williams et al. [75] modeled data from neonatal intensive care units by factorial switching Kalman filters in order to identify several artifactual patterns.

Modeling physiologic variables in steady state can be accomplished by employing low-order autoregressive (AR) models. These models can detect patterns (i.e. deviations from steady state) by comparing incoming observations with confidence intervals for the predications. These models demonstrate potential in detecting clinically relevant patterns in monitoring time series [76]. 
A method by Gather et al. [79] was designed specifically for online identification of outliers and level shifts. The method transformed medical time series into geometric information by considering many consecutive observations as a point in the Euclidean space. Based on this phase-space representation, the method developed can be considered as a general Shewhart control chart for dependent data. This method, AR models, and dynamic linear models were compared in [80] and [81] and the results showed that a single method cannot sufficiently meet all requirements for univariate pattern detection (i.e. one variable at a time) but instead is seems beneficial to combine different methods and adjust them for a specific set of patterns.

\section{Artificial Intelligence}

Several studies have shown promising results, as in [82], when utilizing rule-based systems developed by using expert knowledge in decision trees. It is a difficult approach to develop but has proven to work effectively for alarm-based diagnoses and for correctly detecting pathological states in [83].

Artificial neural networks have also been employed in medical devices to analyze health data. Chan et al. [84] used an artificial neural network that monitored a home care client's mobility data for deviations from their usual pattern. Chan et al. [85] also used this approach in which infrared movement detectors measured the night activities of elderly subjects suffering from Alzheimer's disease. Maurya et al. [86] discusses recent development of a feed-forward neural network system introduced in [87] for trend analysis. The neural network tries to uniquely identify primitives (i.e. basic shapes) from a data set to form trend descriptions. Other studies have used neural networks to detect 
suspicious patterns in cardiotocogram data [88], specific faults in an anaesthesia breathing circuit [89] and [90], and events in vital sign of paediatric intensive care units [91].

Fuzzy logic has also been employed in alarms systems. It is useful when it is difficult to describe the process of interest with a precise mathematical model and in which "experience and intuition play an important role in decision making" [60]. Fuzzy logic has been used in alarms systems for anaesthesia in [92] and [93], monitoring preterm infants [94], and for alarm validation [95] and [96].

Bayesian networks have been explored in clinical settings such as in intensive care unit monitoring for calculating and updating probabilities for the occurrence of noteworthy events [97]. This type of network can be cumbersome to employ because it needs a large amount of prior information. This information helps define and quantify, in terms of conditional probabilities, the dependencies between the different variables [60].

The research in this dissertation compares a statistical approach, a segmentation algorithm, to an artificial intelligence method, a feed-forward neural network, for accuracy in trend detection.

\subsubsection{Introduction to Artificial Neural Networks}

Artificial neural networks mimic the human neural network and were created to be used as decision support system models [98]. Artificial neural networks have shown successful implementation in many application areas and was therefore, chosen as one of the decision support tools in this research.

Artificial neural networks are modeled from biological systems and are composed of simple elements operating in parallel. The connections between the elements greatly influence the function of the network. By adjusting the values of the connections between 
the elements, also known as weights, the neural network can be trained to perform a specific function [99].

Neural networks are most often adjusted, or trained, in order for a specific input to lead to a specific target output. Such is the case in supervised learning in which the network is adjusted, by comparing the output and target, until the network's output equals the target (see Figure 2.4) [99]. Changes are made to the network's weights and biases based on the output targets. Once this learning process is complete, it is hoped that the artificial and real outputs are close enough. This will render the outputs useful for all sets of inputs likely to be encountered in practice [100].

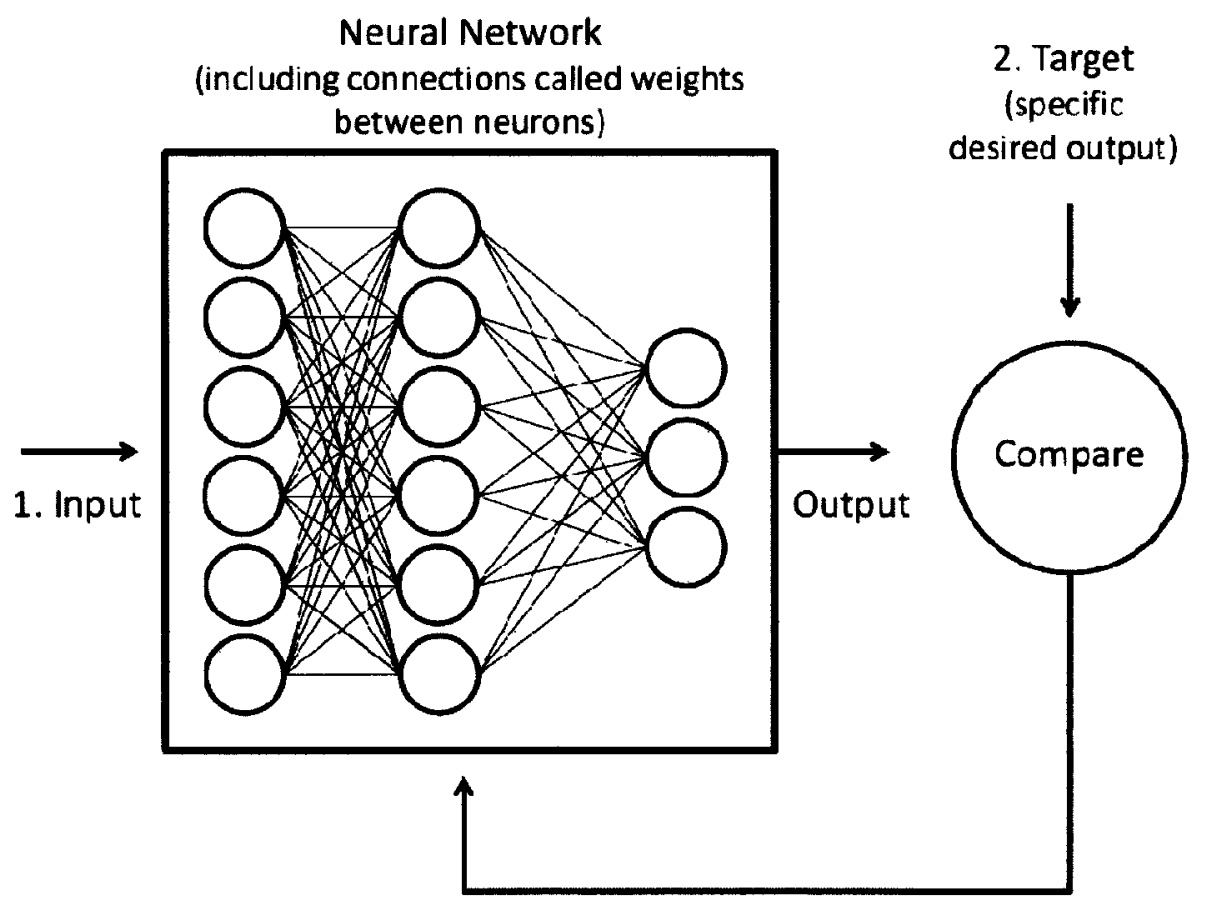

3. Adjust weights using Error

(Desired - Actual)

Figure 2.4: Schematic of supervised learning (adapted from [99]) 
Artificial neural networks are nonlinear statistical models. They are typically represented by a network diagram and are two-state regression or classification models. For example, in the case of regression, typically $K=1$ and there is only one output unit at the top, $Y_{1}$. Nevertheless, it is possible for these networks to handle multiple quantitative responses in a flawless fashion [100].

From linear combinations of the inputs, derived features $Z_{m}$ are created and the target $Y_{k}$ is modeled as a function of linear combinations of $Z_{m}$,

$$
\begin{aligned}
& Z_{m}=\sigma\left(\alpha_{0 m}+\alpha_{m}^{T} X\right), m=1, \ldots, M, \\
& T_{k}=\beta_{o k}+\beta_{k}^{T} Z, k=1, \ldots, K, \\
& f_{k}(X)=g_{k}(T), k=1, \ldots, K,
\end{aligned}
$$

Where $Z=\left(Z_{1}, Z_{2}, \ldots, Z_{M}\right)$, and $T=\left(T_{1}, T_{2}, \ldots, T_{k}\right)$.

Most often, the activation function $\sigma(v)$ in (1) is chosen to be the sigmoid function $\sigma(v)=\frac{1}{\left(1+e^{-v}\right)}[100]$. There are a number of different types of functions that can be used in a network in order to limit the amplitude of the output. Table 2.2 introduces the most commonly used.

Table 2.2: Commonly used neural network transfer functions [99]

\begin{tabular}{|c|l|}
\hline Transfer Function & Description \\
\hline Hard limit & $\begin{array}{l}\text { The hard limit transfer function returns a 0 or a 1. A neuron which } \\
\text { uses this transfer function produces a 1 if the net input into the } \\
\text { function is equal to or greater than 0, else it produces a 0. This } \\
\text { transfer function divides the input space into two regions. }\end{array}$ \\
\hline Linear Transfer & $\begin{array}{l}\text { The linear transfer function calculates the neuron's output by simply } \\
\text { returning the value passed to it. This neuron can either be trained to } \\
\text { learn an affine function of its inputs or for a nonlinear function, find a } \\
\text { linear approximation. }\end{array}$ \\
\hline Log-Sigmoid Transfer & $\begin{array}{l}\text { This transfer function is often used for multilayer networks. As a } \\
\text { neuron's net input goes from negative to positive infinity, this } \\
\text { transfer function produces outputs between 0 and 1. }\end{array}$ \\
\hline
\end{tabular}


A simple neural network diagram (Figure 2.5) can be drawn to help to illustrate the additional bias unit feeding into unit in the hidden and output layers. Hidden units are the units in the middle of the network and are the derived features $Z_{m}$. There can also be more than one hidden layer in a network [100].

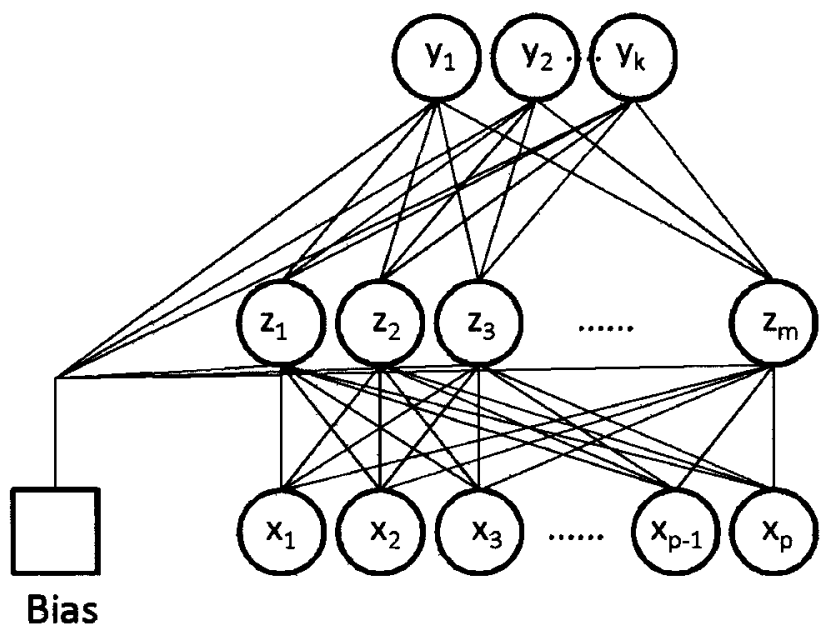

Figure 2.5: Schematic of a single hidden layer, feed-forward neural network (adapted from [100])

Another type of learning is unsupervised learning. This technique can be used in such instances as identifying groups of data and in which there is no measurement of the outcome but only the observation of features $[99],[100]$. The task of this network is to explain how the data is organized and clustered [100]. Changes are not made to the network's weights and biases based on the output targets but rather are a function of the network's current input and output vectors and previous weights and biases [99].

In this type of learning, there is no direct measure of success and instead, heuristic arguments can be used to judge the quality of the results. This has led to many methods of unsupervised learning techniques such as association rules, cluster analysis, and selforganizing maps. 


\section{Chapter 3}

\section{Identifying User Needs and Establishing Requirements}

Before generating any design solutions, a designer needs to understand the key components in order to center the design process around the user. These components were introduced in the previous chapter and include: the users, their needs, and the demands of their work [101]. This chapter summarizes the answers to these components and is split into three sections. Two of these sections are dedicated to front-end analysis, user and function analysis, and the third one to interaction design which presents the usability and user experience goals and design process of and rationale for the decision support system's user interface. Completing these tasks helps to establish the user requirements and will influence the design of the interface.

\subsection{User Analysis}

The user analysis should be completed before any other analysis is conducted. It answers the broad question: who are the product/system users? [34] This user analysis is divided into three sections which include: outlining the target population, providing detailed segmentation, and generating a number of user profiles. 


\subsubsection{Preliminary Segmentation}

The first step in the user analysis is to identify as many potential users as possible and segment these target populations. The results are the following:

- Primary: home care clinicians (nurses, physiotherapists, occupational therapists, case managers)

- Secondary: home care clients, care givers

- Stakeholders: developers, business partners (if the system was designed and built to be sold as a product)

\subsubsection{Detailed Segmentation}

Outlining characteristics of the users allows more effective design to be completed. Types of characteristics that can be included are: demographic (gender, age, physical characteristics, disabilities), experience (training, prior and current job experience, academic experience, computer literacy) [102], and other characteristics such as geographic, psychographic, and benefits and behavioural (e.g. work habits, preferences, literacy, language skills) [103]. Table 3.1 displays the results of detailed segmentation of the primary and secondary potential users of the system.

Table 3.1: Detailed segmentation of primary and secondary users

\begin{tabular}{|l|c|c|}
\hline \multicolumn{1}{|c|}{ Characteristics } & Home care clinicians & $\begin{array}{c}\text { Home care clients, } \\
\text { care givers }\end{array}$ \\
\hline $\begin{array}{l}\text { Experience with technology } \\
\text { and computers }\end{array}$ & Medium & Low \\
\hline Experience with home care & Medium - High & Low \\
\hline $\begin{array}{l}\text { Human, organization } \\
\text { environment }\end{array}$ & $\begin{array}{c}\text { Office, car, home care } \\
\text { client's residence }\end{array}$ & Home \\
\hline $\begin{array}{l}\text { Training, learning, support } \\
\text { opportunities }\end{array}$ & Medium & Low \\
\hline Attitude, motivation & Medium & Medium \\
\hline Ability to master complexity & High & Medium \\
\hline
\end{tabular}




\subsubsection{User Profiles}

This thesis focuses on the primary user groups which includes home care nurses, physiotherapists, occupational therapists, and case managers. In order to validate results from an evaluation of a GUI, subjects from each of these user groups are required. Subjects from the secondary user groups were not recruited for this portion of the research because of ethics approval and time constraints.

Identifying and selecting the sample target subjects for an evaluation will help to determine the user profile which "describes the range of skills of target end-users of a system" [104].The following profiles for each of the user groups were constructed with the help of criteria established by Kushniruk and Patel [104] and Grossman et al. [105].

\section{User Profile - home care nurses}

The roles of the subjects in the workplace, critical skills, and knowledge: professional nursing services, including complex care and specialized services (e.g. assistance with bathing, grooming, bedside care and assistance). They provide wound care, medication administration, IV therapy, ostomy care, pain management and develop treatment plans [106].

\section{User profile - physiotherapists}

The roles of the subjects in the workplace, critical skills, and knowledge: licensed primary health care providers who help the clients with rehabilitation services (speed and degree of recovery from surgery, injuries or general weakness) and provide treatment to clients to make certain they regain their ability to function at home and work. These programs consist of exercises that the client completes in the home with and without the physiotherapist. The type of exercises and treatment plan is determined by how well the client is responding [106]. 


\section{User profile - occupational therapists}

The roles of the subjects in the workplace, critical skills, and knowledge: rehabilitation services in an independent, client centered, community based practice; conduct assessments, formulate recommendations, plan and implement goal-directed treatment programs. They also assess and treat clients with a wide range of developmental, physical, neurological and psychiatric disorders, provide advice on home accessibility, safety and energy conservation, and assist clients to be independent and safe in their homes. This is done through assisting in the implementation of assistive devices such as: walking devices, kitchen aids, safe home assessments, toilet seats, and stair lifts. Other devices include: memory assistance (development of memory retention techniques such as the use of calendars), large digit clocks, phones, and eating devices. The occupational therapists conduct periodic evaluations of how the client is coping in the home and provide changes in care if necessary [106].

\section{User profile - case managers}

The roles of the subjects in the workplace, critical skills, and knowledge: a needs assessment that considers not only the client but also the family, case coordination and management from admission until discharge [106]. As defined by Health Canada [107], case management is:

a collaborative client-driven strategy for the provision of quality health and support services through the effective and efficient use of available resources in order to support the client's achievement of goals related to health life and living in the context of the person and their ability. 


\section{User Profile - General Criteria}

Accountability/relationships: All nurses and occupational therapists are subordinates for the nurse or occupational therapists in-charge. They are also required to report to the case managers for authorization of client services. Specifically, for this work, nurses, physiotherapists and occupational therapists are employed by the home care agency (Saint Elizabeth) while case managers are employed by the Community Care Access Centre.

Tools and job aids: For their employment, the clinicians utilize cell phones and pagers. Their primary sources of communication with clients and providers are through faxing and voice mails. Most of their documentation is paper-based. Computer access is not provided as part of the job and there is no information about computer use at home. Although the clinicians are given work email accounts, they are not provided computer access as part of their work (with the exception of Case Managers). Case managers have better access to emails and computers. Some of them are given laptops when they make home visits.

\subsection{Function Analysis}

This section outlines the basic functions to be performed by the system and presents the results from a focus group conducted with the primary user groups regarding the general concepts of the system.

\subsubsection{System Functions}

A function analysis is performed once the population of potential users has been identified. These "functions represent general transformations of information and system state that help people achieve their goals but do not specify particular tasks" [34]. The three main functions of system include: 
- Analyze bio-mobility information from remote-monitoring sensors

- Display the information in a way that is meaningful to the potential users

- Provide decision-support capabilities

\subsubsection{Methodology of the Focus Groups}

Focus groups were conducted with the four different types of home care clinicians in order to determine which client groups would benefit from bio-mobility monitoring. The clinicians involved were Saint Elizabeth Health Care (Toronto) nurses, occupational therapists, physiotherapists, and case managers. In total, twenty health care professionals participated in three focus groups (the occupational therapists and physiotherapists participated in the same focus group).

All of the sessions were facilitated by a member of the research team. One of the aims of conducting the focus groups was to ensure that the technologies being developed would meet the needs of the homecare clinicians and their clients. Discussions with the home care clinicians included identifying client groups for whom it would be helpful to have remote access to clinical information to support care and their preferences in retrieving client related data. Other questions for the clinicians inquired about technologies that might be useful for their practice, types of information that would assist in care giving, and types of information resources that the homecare clinicians may need in order to support clinical decisions and their work in general.

At some point in each of the focus groups, two presentations were made on currently available monitoring technologies at Wilfred Laurier University (WLU) and Carleton University (CU). WLU's presentation introduced the use of a GPS-supporting monitoring system to track clients' daily activities and health conditions. CU's 
presentation addressed current unobtrusive smart home technology sensors such as a bed pressure sensitive mat. Participants of the focus groups were asked to provide input on if and how these technologies would be useful to their practice. These presentations were also meant to help in generating ideas from and discussion with the health care clinicians.

\subsubsection{Results from the Focus Groups}

In order to capture all the discussions, each session was tape-recorded and then transcribed. Content analysis was conducted jointly with nursing students from University of Toronto to discover common themes that described appropriate use cases for the system. The research team also analyzed the transcripts in order to identify specific client populations. Populations were chosen if the team believed that they would benefit from the GPS-supporting monitoring system and data of the bed pressure sensitive mat in combination with a decision support system.

There were four specific themes explored in the focus groups. These themes included:

1. The client groups for whom it would be beneficial to have remote monitoring technologies;

2. The type of client data or resources that would be helpful to clinicians;

3. The format and frequency of data to be received by the clinicians; and

4. The potential users of remote monitoring technologies.

Common opinions and thoughts emerged from the focus groups. The home care clinicians indicated that three main client groups would benefit the most from remote monitoring technologies: clients with mobility issues (e.g. at risk for falls, who are homebound, post-operative, or in rehabilitation), with cognitive impairments (e.g. 
Alzheimer's, dementia, risk for wandering), and those who require continuous monitoring due to high-risk conditions.

Another common finding from the focus groups was the need for a wide variety of client data (e.g. both outdoor and indoor activities). For the clinicians, this would be considered helpful because the need for specific client data is context dependent. The clinicians found this necessary because the type of data needed to support decision making varies with the clinical expertise and role of the caregiver. The home care clinicians also indicated that they would prefer graphical representation of data to numeric. They also indicated that important features for the interface to display were historical trends and some form of alert if the client was deviating from their norm.

The home care clinicians also indicated other end user groups besides themselves. These included other formal health care providers and informal care providers such as family members.

Detailed findings of the focus groups can be found in Table 3.2. The findings for the occupational therapists and physiotherapists are combined because they stem from the same focus group session. 
Table 3.2: Key findings from the focus groups with home care clinicians

\begin{tabular}{|c|c|c|c|c|}
\hline & $\begin{array}{l}\text { Client groups for whom it would be } \\
\text { beneficial to have remote monitoring } \\
\text { technologies }\end{array}$ & $\begin{array}{l}\text { Type of client data or resources } \\
\text { that would be helpful }\end{array}$ & $\begin{array}{l}\text { Format and frequency } \\
\text { of data to be received }\end{array}$ & $\begin{array}{l}\text { Potential users of remote } \\
\text { monitoring technologies }\end{array}$ \\
\hline 密 & $\begin{array}{l}\text { - } \text { Clients with mobility issues } \\
\text { alients who are home-bound and live } \\
\text { - } \text { Rehab clients } \\
\text { - } \text { Clients who are bedridden with pressure } \\
\text { - Clients who are at risk for wandering (i.e. } \\
\text { dementia, Alzheimer's disease) } \\
\text { - Psychiatric patients } \\
\text { - Dialysis clients and clients with } \\
\text { Congestive Heart Failure } \\
\text { - Sedentary diabetics } \\
\text { - Post-op clients (i.e. Hernia repair) } \\
\text { - Clderly clients with functional decline } \\
\text { breathing } \\
\text { - Pain management patients } \\
\text { - Clients with malnutrition and/or } \\
\text { dehydration } \\
\text { - Clients who are non-compliant with } \\
\text { medications } \\
\text { Paediatric clients such as Preemie }\end{array}$ & $\begin{array}{l}\text { - Patient history and records } \\
\text { - Mobility information } \\
\text { - Information to assess equipment } \\
\text { needs } \\
\text { - Information to assess } \\
\text { medication compliance } \\
\text { - Information to locate missing } \\
\text { clients } \\
\text { - Weight } \\
\text { - Vital signs (i.e. heart rate, } \\
\text { breathing, blood pressure) } \\
\text { - Blood sugar levels } \\
\text { - Lab results and diagnostic tests } \\
\text { (i.e. CT scan results) } \\
\text { - Intake and output } \\
\text { Pain management data }\end{array}$ & $\begin{array}{l}\text { Data that shows } \\
\text { trends and changes } \\
\text { - Summary of } \\
\text { information } \\
\text { - Visual data (i.e. } \\
\text { graph) }\end{array}$ & $\begin{array}{l}\text { - Nurses are unsure of its } \\
\text { potential application to } \\
\text { their practice } \\
\text { - Occupational therapists } \\
\text { and Physiotherapists } \\
\text { - Volunteer s(i.e. friendly } \\
\text { visitors) } \\
\text { - Case managers } \\
\text { - Physicians }\end{array}$ \\
\hline
\end{tabular}




\begin{tabular}{|c|c|c|c|c|}
\hline & $\begin{array}{l}\text { Client groups for whom it would be } \\
\text { beneficial to have remote monitoring } \\
\text { technologies }\end{array}$ & $\begin{array}{l}\text { Type of client data or resources } \\
\text { that would be helpful }\end{array}$ & $\begin{array}{l}\text { Format and frequency } \\
\text { of data to be received }\end{array}$ & $\begin{array}{l}\text { Potential users of remote } \\
\text { monitoring technologies }\end{array}$ \\
\hline 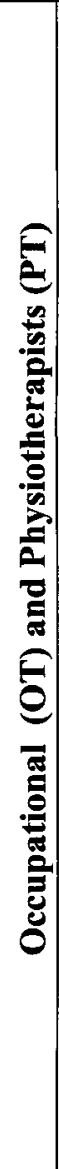 & 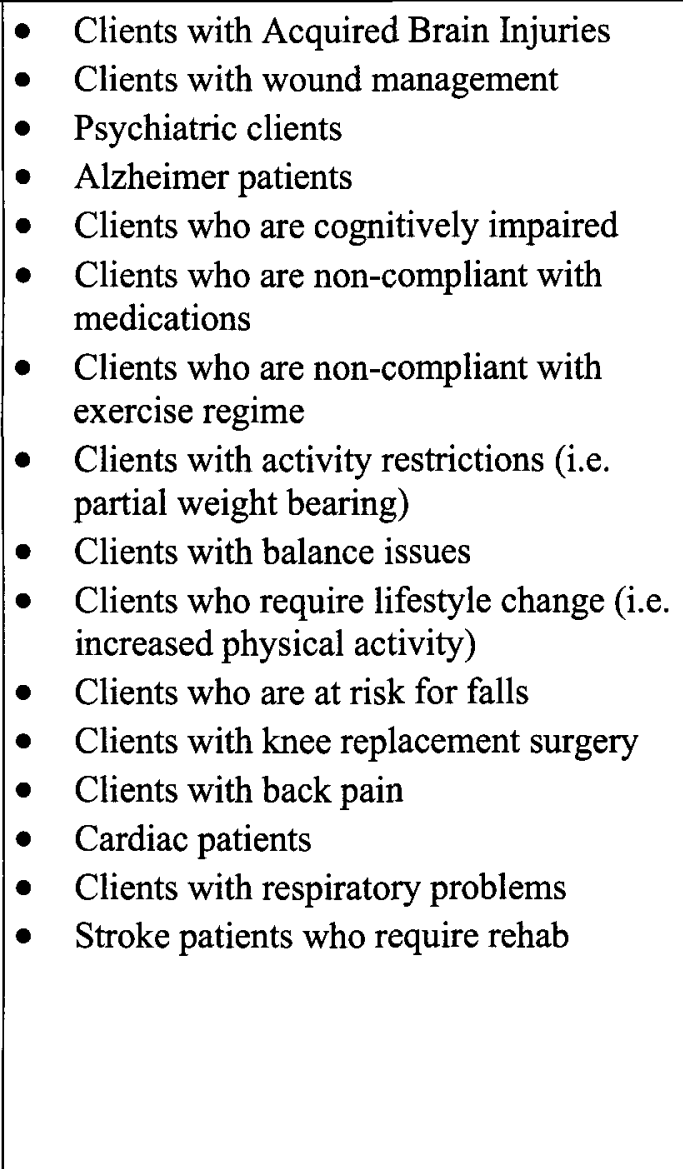 & $\begin{array}{l}\text { Decision-support data for } \\
\text { pressure relieving education to } \\
\text { prevent pressure sores } \\
\text { - Information to track missing } \\
\text { patients } \\
\text { - Alert system regarding kitchen } \\
\text { safety (i.e. use of stove, } \\
\text { microwave) } \\
\text { - Data about how patients put } \\
\text { weight while walking } \\
\text { - Reminder or coaching system to } \\
\text { ensure exercises are performed } \\
\text { by clients } \\
\text { - Data that monitor activity level } \\
\text { and tolerance } \\
\text { - Data that track client regarding } \\
\text { scheduling, pacing and planning } \\
\text { of activities } \\
\text { Data that indicate why, how and } \\
\text { where clients fall (i.e. backward } \\
\text { vs. forward fall) } \\
\text { Data about the speed and } \\
\text { distance of walking } \\
\text { Feedback about client progress } \\
\text { towards activity goals (i.e. } \\
\text { getting better or worse) }\end{array}$ & 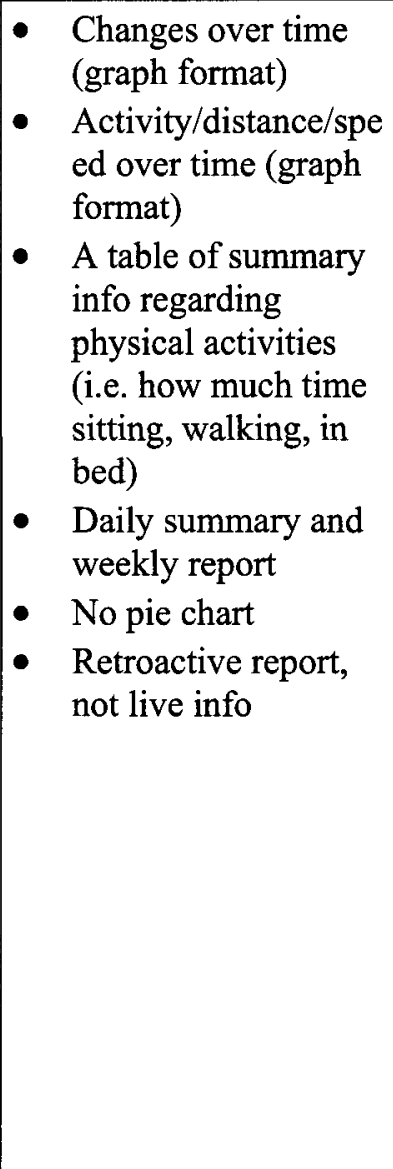 & $\begin{array}{l}\text { Family members and } \\
\text { other caregivers (i.e. } \\
\text { personal support worker } \\
\text { OT would benefit from } \\
\text { monitoring client home } \\
\text { safety and integration of } \\
\text { lifestyle change } \\
\text { PT would benefit from } \\
\text { fall prevention and } \\
\text { monitoring } \\
\text { improvement and } \\
\text { progress about activity } \\
\text { level }\end{array}$ \\
\hline
\end{tabular}




\begin{tabular}{|c|c|c|c|c|}
\hline & $\begin{array}{l}\text { Client groups for whom it would be } \\
\text { beneficial to have remote monitoring } \\
\text { technologies }\end{array}$ & $\begin{array}{l}\text { Type of client data or resources } \\
\text { that would be helpful }\end{array}$ & $\begin{array}{l}\text { Format and frequency } \\
\text { of data to be received }\end{array}$ & $\begin{array}{l}\text { Potential users of remote } \\
\text { monitoring technologies }\end{array}$ \\
\hline 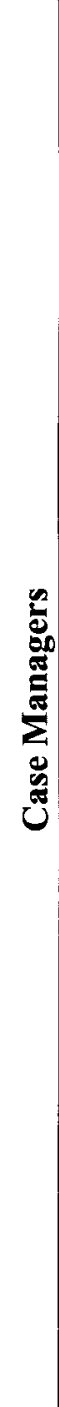 & $\begin{array}{l}\text { - Clients with orthopaedic surgeries (i.e. } \\
\text { hip replacement) } \\
\text { - Home-bound clients who live alone } \\
\text { - } \text { impaired } \\
\text { - Clients with Parkinson's disease } \\
\text { - Clients at high risk for long-term care } \\
\text { placement } \\
\text { - Seniors who lack of support system } \\
\text { - Pain management clients } \\
\text { - Clients with issues of incontinence } \\
\text { - } \text { ulcers } \\
\text { - Clients at risk for falls } \\
\text { - medients who are non-compliant with } \\
\text { - Clients who needs monitoring of } \\
\text { nutritional needs } \\
\text { - Clients with weight management } \\
\text { Paediatric clients }\end{array}$ & $\begin{array}{l}\text { - } \\
\text { - } \text { - } \text {-Hedient baseline data history } \\
\text { clients/ neighbourhood (for } \\
\text { nurse safety) } \\
\text { - Mobility information (i.e. } \\
\text { speed, frequency, distance) } \\
\text { - Intensity of physical activity } \\
\text { (i.e. walking, running) } \\
\text { - Nutritional information } \\
\text { Information to support best } \\
\text { practices/ interventions on } \\
\text { chronic disease management } \\
\text { (i.e. diabetic care) } \\
\text { - Decision support info alerted } \\
\text { clinicians when data are out of } \\
\text { normal range } \\
\text { - Pain scale } \\
\text { - Information to support resource } \\
\text { allocation of home services } \\
\text { - Information to support } \\
\text { scheduling and adjusting of } \\
\text { drug dosages } \\
\text { - Prompted voiding alerts for } \\
\text { incontinent clients } \\
\text { - Reminder system that provide } \\
\text { cues when clients need to be } \\
\text { repositioned } \\
\text { - Information to support quality } \\
\text { assurance of service providers } \\
\text { Best practices on fall prevention }\end{array}$ & 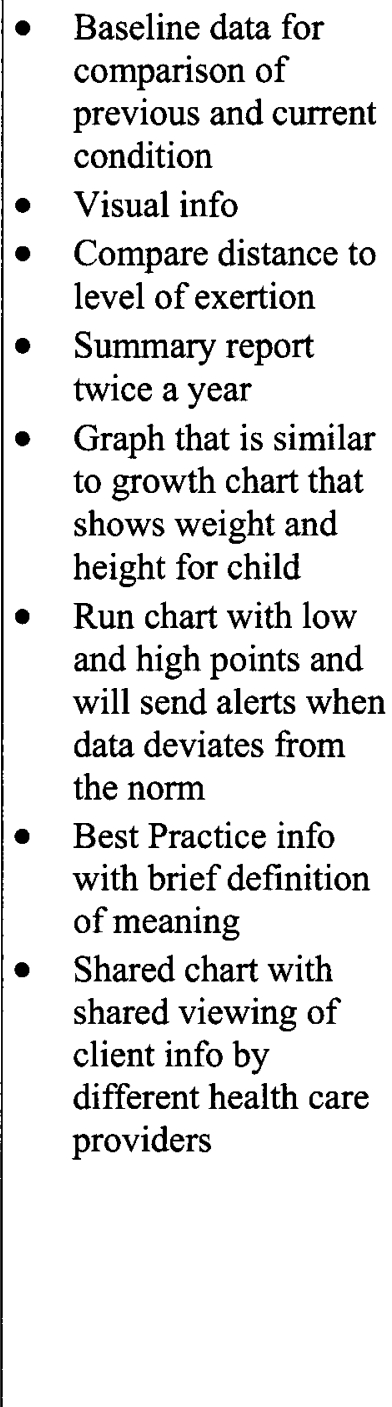 & $\begin{array}{ll}\text { - } & \text { Personal support service } \\
\text { workers } \\
\text { - } & \text { Family caregivers } \\
\text { - } & \text { Front-line nurses } \\
\text { - Physicians (i.e. to } \\
\text { monitor and adjust } \\
\text { medication dosages } \\
\text { - CCAC case managers } \\
\text { - } \text { (clinical vs. monitoring } \\
\text { role) }\end{array}$ \\
\hline
\end{tabular}




\subsection{Interaction Design}

After the research team conducted focus groups with home care clinicians (nurses, physiotherapists, occupational therapists, and case managers) about a proposed client monitoring system, the information acquired had to be compiled in order to begin designing prototype graphical user interfaces (GUIs) for a client monitoring and decision support system. The research team agreed that a human-centered design approach was appropriate because it emphasizes user involvement and applies an iterative approach to the design and testing of the prototype. Therefore, the goal of this step in the design process was to prepare to conduct a usability test on prototype GUIs of the system. The results from the testing would then help resolve conceptual and detailed design issues regarding the prototype displays.

This section outlines the usability goals and presents the design process and rationale of the interactive interface.

\subsubsection{Usability and User Experience Goals}

Identifying usability goals allows a designer to consistently assess various aspects of an interactive system and the user experience. Many user experience goals may also be identified in interaction design. These goals may be subjective qualities and discover how a system feels to a user. These types of goals relate to how users experience an interactive product from their perspective rather than the previous type of goals which assess how useful a system is from its own perspective [101]. Table 3.3 outlines the general usability and user experience goals of the GUIs. 
Table 3.3: Usability and user experience goals of the graphical user interfaces

\begin{tabular}{|l|l|}
\hline \multicolumn{2}{|l|}{ Usability and User Experience Goals and the Interaction and Outcome Criteria } \\
\hline Learning & Minimum learning required \\
\hline Efficiency & Minimum number of actions \\
\hline Efficiency & Should be able to work fast - provide shortcuts \\
\hline Reduce cognitive load & Users should not have to remember anything \\
\hline Reduce cognitive load & Direct manipulation: visible objects, visible choices \\
\hline Error prevention; learning & Clear display of information \\
\hline $\begin{array}{l}\text { Error reduction and } \\
\text { prevention }\end{array}$ & $\begin{array}{l}\text { Use simple, natural dialog; eliminate extraneous words / } \\
\text { graphics }\end{array}$ \\
\hline Experience & $\begin{array}{l}\text { Should function easily to reduce any high frustration level } \\
\text { which would degrade the user performance and be aiding and } \\
\text { not distracting from the job at hand }\end{array}$ \\
\hline Product image & Should look personal \\
\hline
\end{tabular}

\subsubsection{Design Process of and Rationale for the User Interface}

Producing mock-up interfaces, also known as prototypes, early in the design cycle supports interface and interaction design and usability testing. The prototypes generated for this stage were meant to be crude approximations of what the final product may be like. The prototypes were designed to have the look and feel of the final product but not have the full functionality. There are a number of advantages outlined by [34] on the use of prototypes during the design process some of which include:

- Confirming insights gathered during the front-end analysis (e.g. user and function analysis)

- Support of the design team in making ideas concrete

- Support of the design team by providing a communication medium

- Support for usability testing by giving users something to react to and use Finding usability problems early in the design cycle is crucial to a successful design. Reducing the amount of usability problems helps to: reduce learning and training time, support the user to focus on the task, reduce user errors, improve productivity, and enhance user experience and acceptance. 
Members of the research team (faculty and students) met in Ottawa in June 2009 to review the first drafts of the GUIs created. In the following months, two possible versions of a prototype user interface were developed that would encompass the data outputs of the monitoring technologies at Carleton and Laurier. The following discusses the rationale of the interface features as guided by interface design principles, the focus groups' results and system functions.

\section{Navigation}

Each GUI version consists of three levels of data in a 'dig-down' format - each subsequent level of data being more detailed than the previous. Three levels of data were thought to be appropriate so as to ensure easy navigation and still provide enough detailed client health information. This also follows the Information Visualization Mantra: overview first, zoom and filter, details on demand [108].

The first level of data, the "overview", for the clinicians is a graphic summary of a client's data as the home page. The aim is to provide a clear screen with links to other major navigation points. Another navigation option is the basic set of links presented at the top of every page. These links are formed as a button bar which meets an important criteria of "efficient" by "offering multiple choices in a small space, and [being] predictable, it is always there at the top of the page, and provides a consistent graphic identity" [109] throughout the interface.

The second level of data can be accessed from the home page by either selecting an option from the button bar or by selecting a specific piece of health data. The second level provides more details regarding all the pieces of health data on the interface. For example, the home pages for both prototypes (Figure 3.1 and Figure 3.4) are set-up to 
present averages of the data collected from a home care client over the last seven days. The second level of data (Figure 3.2, Figure 3.3, Figure 3.5 and Figure 3.6) provides the cliniancs with a breakdown of daily activity and the third level provides hourly.

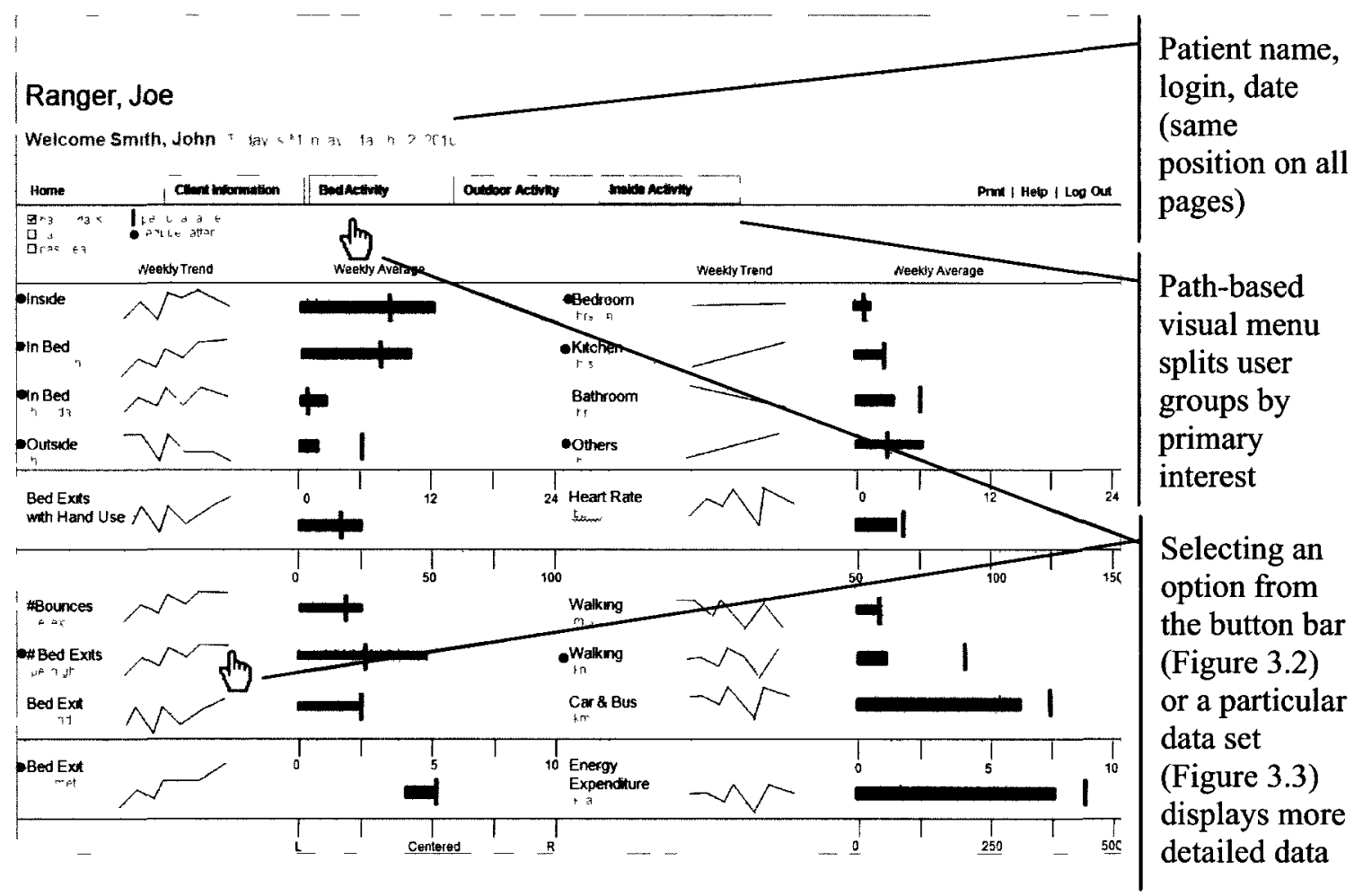

Figure 3.1: Version 'A' home page 


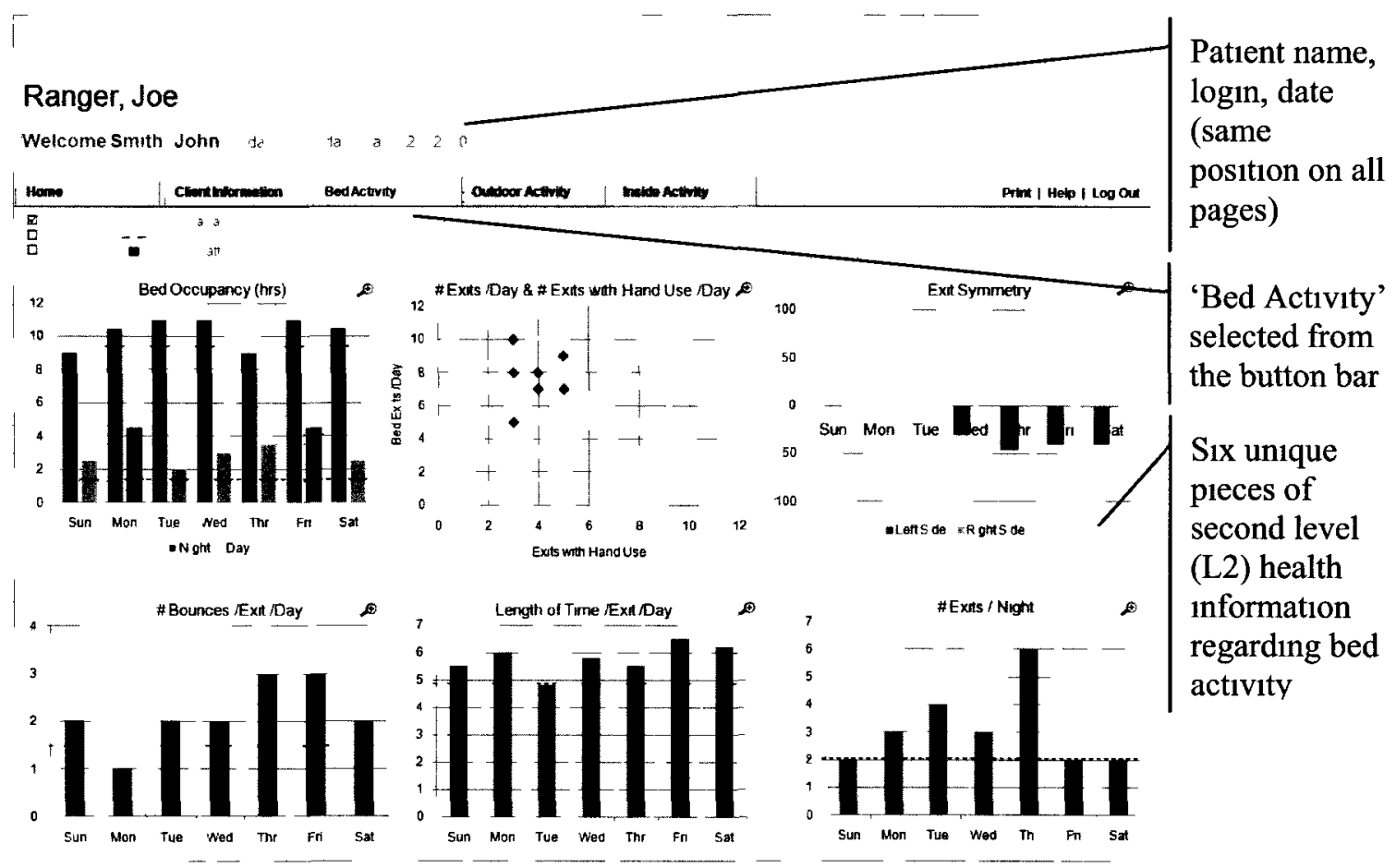

Figure 3.2: Version ' $A$ ' second level (L2) information reached from the button bar

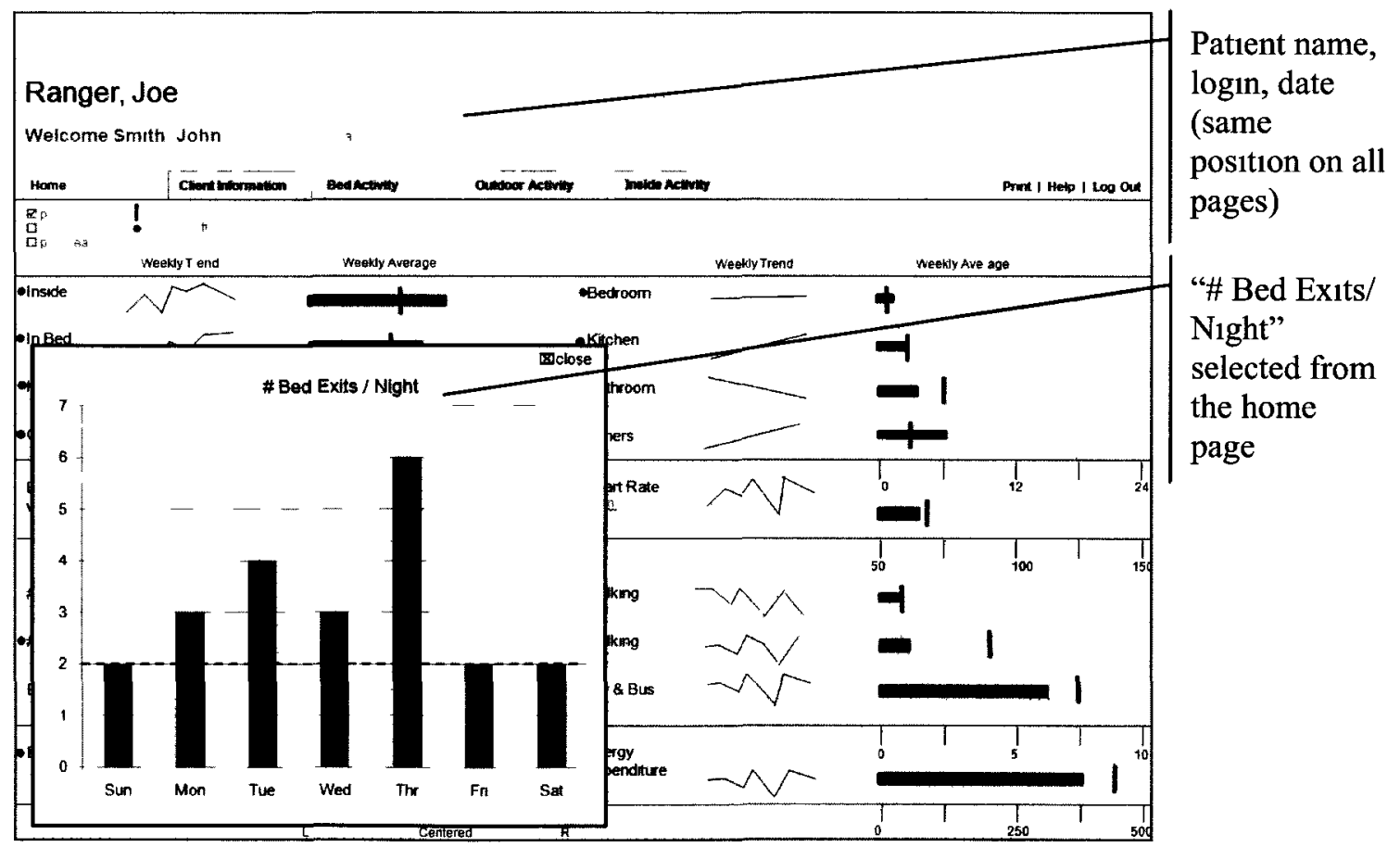

Figure 3.3: Version ' $A$ ' second level (L2) information reached from selecting a piece of health data from the home page 


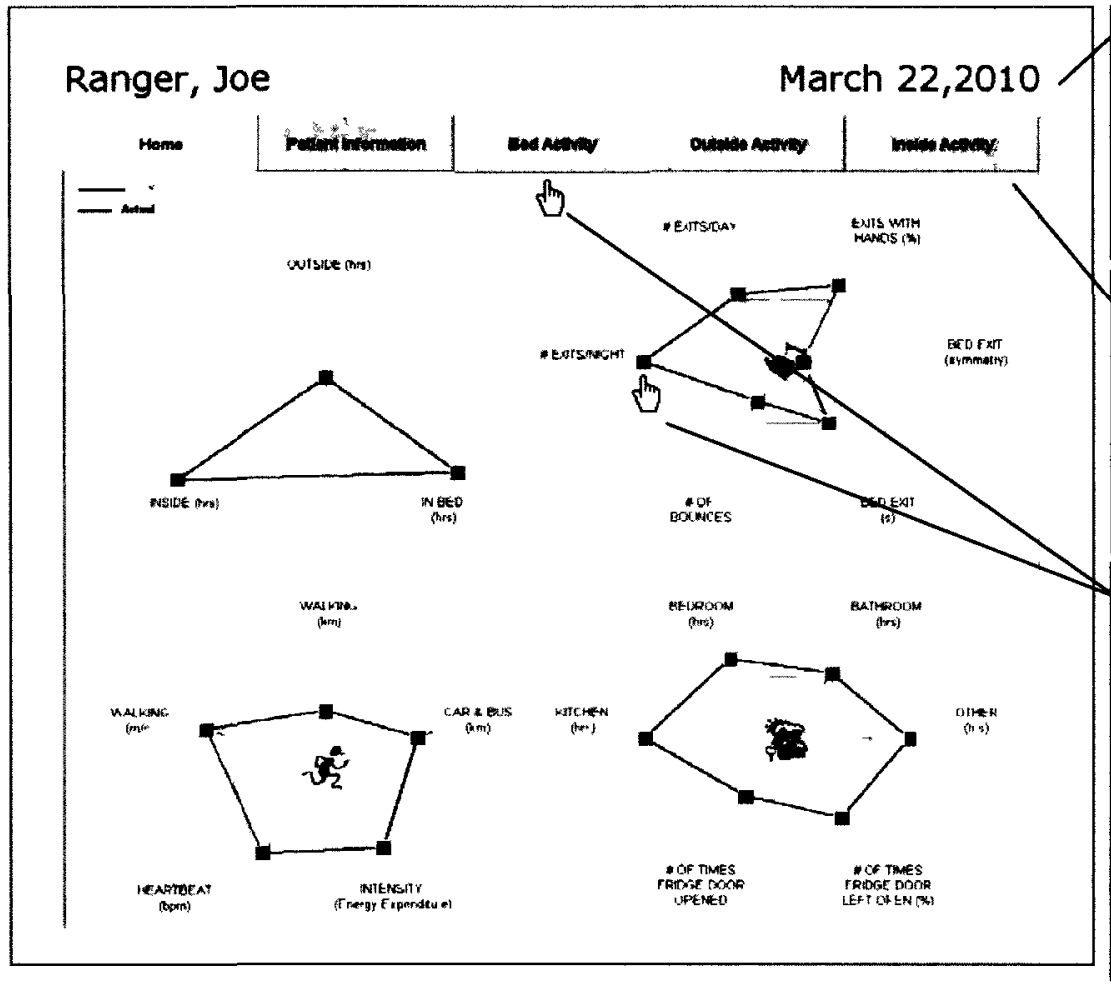

Patient name, date (same position on all pages)

Path-based visual menu splits user groups by primary interest

Selecting an option from the button bar (Figure 3.2) or a particular data set

(Figure 3.3) displays more detalled data

Figure 3.4: Version 'B' home page

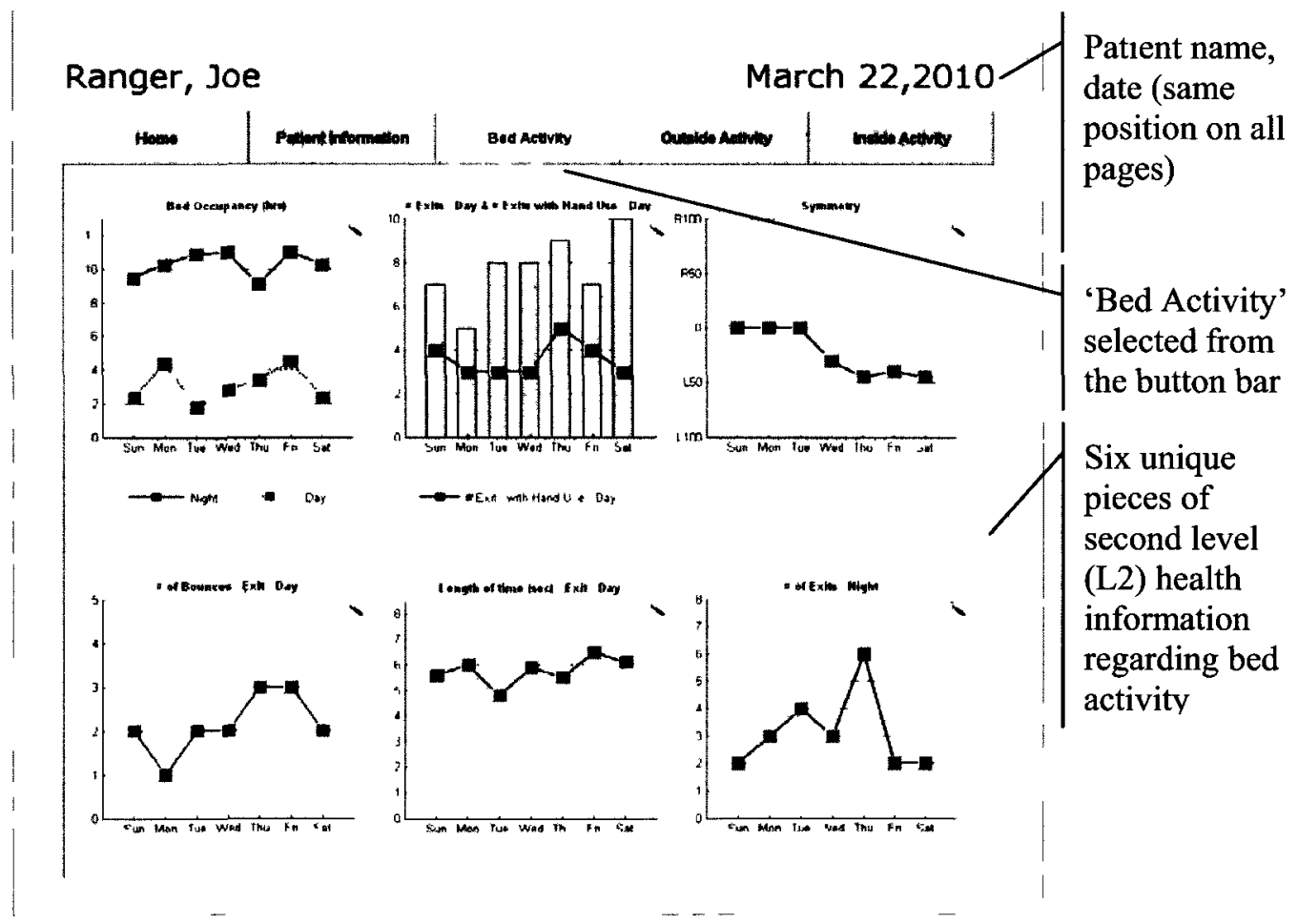

Figure 3.5: Version ' $B$ ' second level (L2) information reached from the button bar 


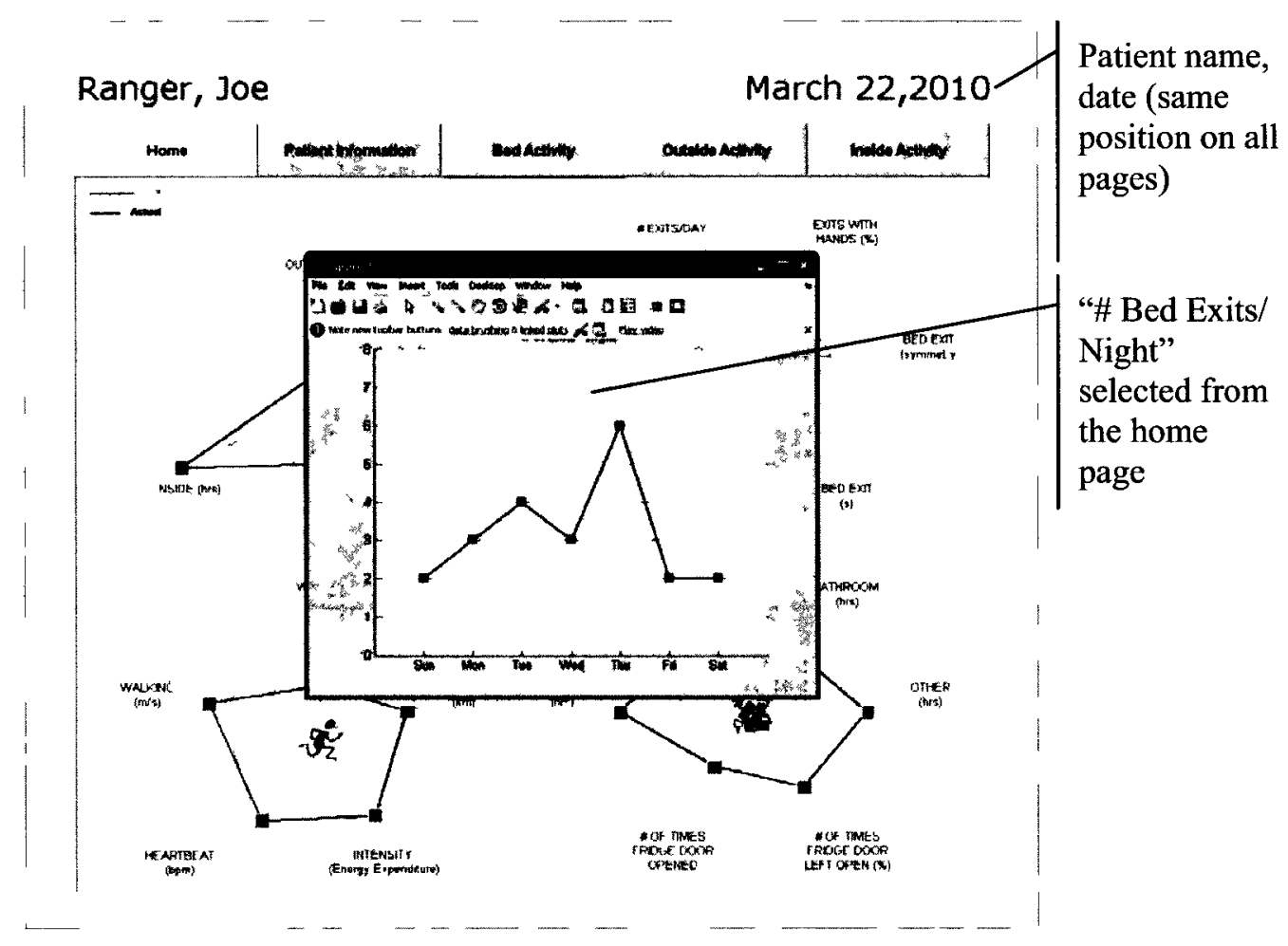

Figure 3.6: Version 'B' second level (L2) information reached from selecting a piece of health data from the home page

The prototypes help address the clinicians' needs regarding the client groups identified in the focus groups by employing the button bar. Clients with mobility issues can be monitored quickly by viewing "Bed Activity" and clients with cognitive impairments can be monitored by "Inside Activity" and "Outside Activity". The status of clients who require continuous monitoring due to high risk conditions can be viewed through the "Bed Activity" data.

This type of data sets also helps address the need for a wide variety of client data. The prototypes display health data encompassing the client's activity both in and out of their residence. 


\section{Hierarchy}

Remembering that users want to acquire information from the interface in the fewest possible steps, an efficient hierarchy of information needed to be determined. It has been established that users prefer "menus that present at least $5-7$ links and that they prefer a few very dense screen choices to many layers of simplified menus" [109]. Therefore, five basic steps outlined by [109] were followed in organizing the information for the GUIs:

- Divide content into logical units (e.g. inside and outside client data, bed pressure sensor mat and GPS- monitoring data)

- Establish a hierarchy of importance among the units (moving from the most general overview of the site (the home page), down through increasingly specific submenus and content pages)

- Use the hierarchy to structure relations among units

- Build an interface that closely follows the information structure

- Analyze the functional and aesthetic success

\section{Graphical representation of historical data}

Two of the key needs from the focus groups were graphical representation of data and historical data trends of their clients. This type of data can be presented in various formats including box plots, scatter plots, data distribution charts, curves, volume visualization, surfaces or link graphs [110].

Within the interface, there also consists a variety of such plots. The plots used on Version ' $A$ ' include sparklines, bullet and bar graphs. Figure 3.7 is an example of the 
manner in which parameters on the homepage of Version ' $A$ ' are presented. This particular parameter indicates the number of hours a home care client has been outside.

\section{Weekly Trend Weekly Average}

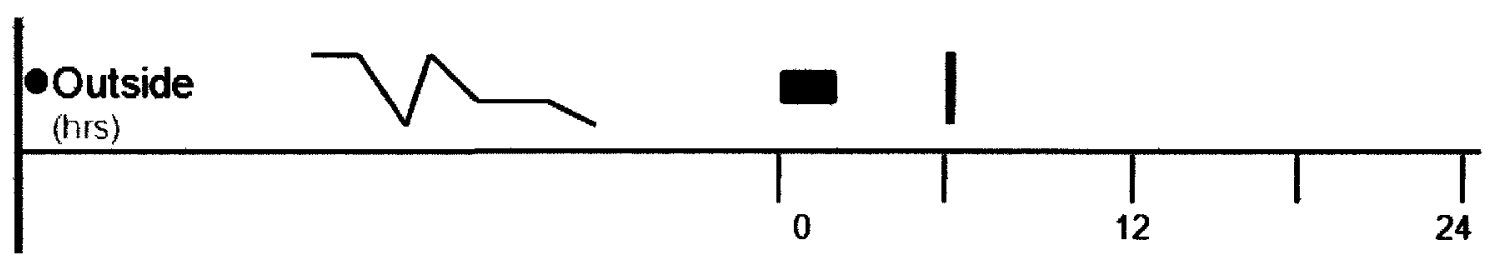

Figure 3.7: Representation of parameters on the homepage of Version 'A' (adapted from [111])

The 'sparkline', a type of line graph, is meant to give historical information [111]. The graph conveys the client's historical trend and patterns of the past week or month, whatever time frame the clinician selects. The 'bullet graph', a type of bar graph, is meant to provide precise information of the weekly average along with the client's personal target (the vertical black line). A legend indicating the meaning of the vertical black line and the red dot (to be discussed later on) is displayed on the homepage above all the parameters.

The plots used within Version ' $\mathrm{B}$ ' include polar displays and scatter plots. Polar displays are a unique case of a configural graphic that are "created by orienting the axes of several variables so they originate from the same point" [112]. Polar displays are created by normalizing the data and setting the normal to the same distance on each axis to produce the polar stars. Therefore, the polar display "forms a regular polygon when the system state is normal and an irregular polygon in abnormal states" [112]. This allows the clinicians to identify the differences between abnormal and normal easily. The limitation of the polar display is that it is difficult to show time history information on the graph; although in some cases arrows have been used to indicate the direction and rate of 
change. The graphic is improved by showing the client's personal target as the regular polygon. An example of a polar display from Version ' $B$ ' for bed activity is shown below (Figure 3.8).

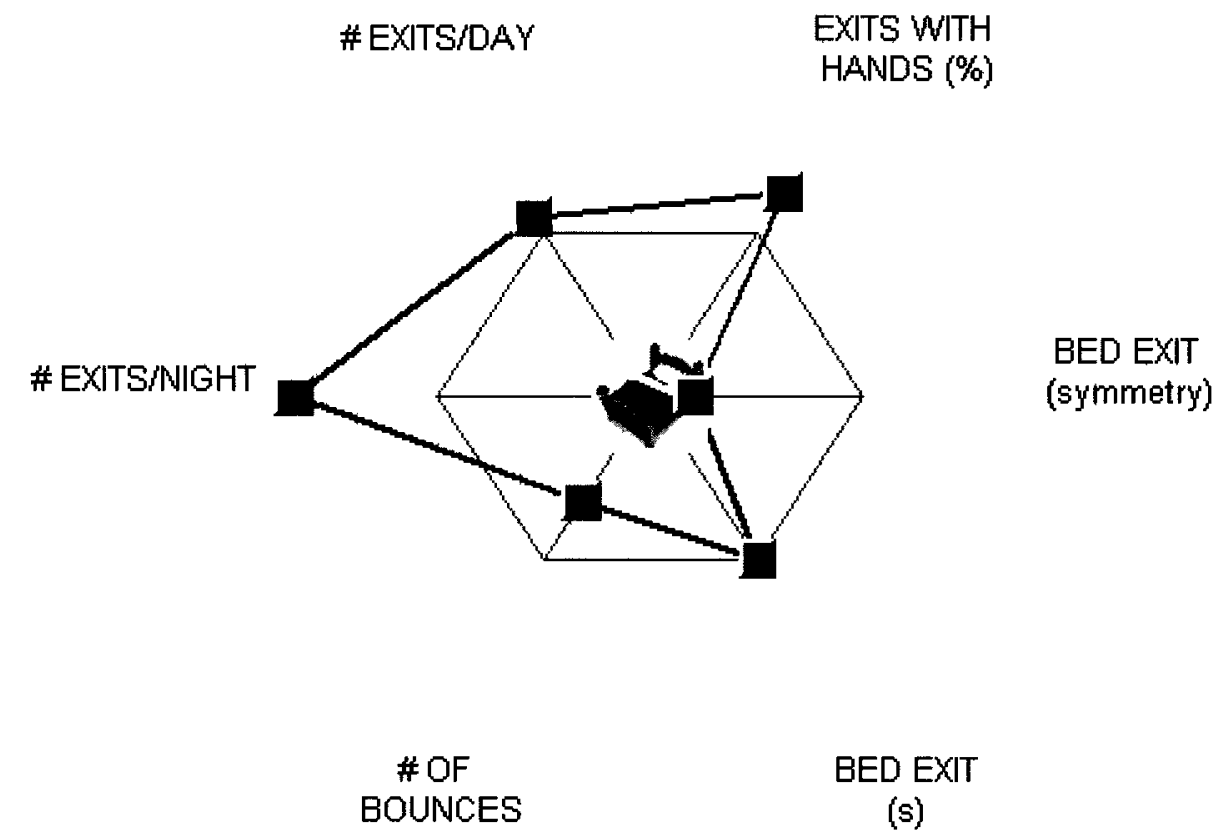

Figure 3.8: Example of a polar display for Version ' $B$ '

Scatter plots refer to the visualization of data items according to two axes, namely $\mathrm{X}$ and $\mathrm{Y}$ values. According to [113], the scatter plot is the most popular visualization tool, since it can help find clusters, outliers, trends and correlations. Scatter plots are the most prevalent plot type at the second level, once a user has requested more detailed information from the home page, of Version ' $\mathrm{B}$ '.

\section{Alerts}

A third result from the focus groups was that the clinicians wanted alerts if a client was deviating from their norm. It was chosen to only have one level of alert for the first iteration of GUI design (i.e. not a caution zone as well) and represent it with a shape and the colour red. This was the decision because a scale of alerts creates the problem of 
defining different levels of emergent/ urgent situations and these were unknown at the time since what the technologies could monitor, were new to the clinicians.

\section{Colour}

Choosing a colour scheme is also an important feature and directly affects the items discussed above. Choosing colours that are analogous to one another, meaning they are close to one another on the colour wheel, creates a subdued, quiet effect on the design. Choosing colours which are complementary to one another, meaning they are opposite to each other on the wheel, creates a vivid scheme that demands attention. Choosing a polychrome design, meaning many colours from all over the colour wheel, needs to be chosen carefully so that the colours do not clash. Choosing a neutral scheme, one that contains black, grey and white, was the choice for both prototypes so that red alerts could pop out well from the rest of the data [114].

Another important aspect to bear in mind is the Five Shade Rule. This rule states that the human eye can only distinguish between five shades of the same colour (hue). Therefore, the neutral scheme with only one level of alert colour obeys by this rule. This choice also follows good practice in that colour should be used meaningfully, not arbitrarily or gratuitously [114]. 


\section{Chapter 4}

\section{Graphical User Interface Usability Test}

This chapter outlines the steps taken to conduct a usability test with both graphical user interface prototypes introduced in Chapter 3. It begins by identifying the evaluation objectives, sample selection and study design. The appropriate experimental tasks and evaluation environment are also discussed. A qualitative analysis of the results from the test is presented followed by the interpretation of findings.

\subsection{Identification of Evaluation Objectives}

The objectives for conducting an evaluation of two GUI prototypes as taken from [104] and [109] include the ability to:

1. Assess system functionality and usability (e.g. learning, navigation, and orientation)

2. Acquire feedback for refining the prototypes

3. Identify human-computer interaction issues (e.g. how should client status indicators be designed (are the indicators detectable/ noticeable)?)

4. Assess the impact of the new information technology on home care practice The prototypes created should incorporate enough pages to assess accurately what it is like for the clinicians to navigate from page to page but should not be so complex or 
elaborate that there is too much investment in one design at the expense of exploring alternatives [109].

By defining specific usability goals, as discussed in sub-section 3.3.1, and by prioritizing objectives, it allows for the creation of evaluation scenarios and tasks to be developed. These generated scenarios and tasks can then be used in usability tests. The results from usability tests inform the designers whether their concerns were valid, what measures can help determine if participants were have trouble completing the tasks, and identify what design changes are needed to meet the objectives.

\subsection{Sample Selection and Study Design}

The identification and selection of target subjects for the evaluation and their defined user profiles are outlined above in section 3.1. These users include: home care nurses, physiotherapists, occupational therapists, and case managers. The four user groups are representative of the end users of the GUI.

It has been established that a small number of subjects for an evaluation involving cognitive analysis can be sufficient for soliciting quality feedback [115] and [116]. A group of $8-10$ subjects, who are representative of the target users of the system being evaluated, can provide a substantial amount of information; up to $80 \%$ of the surface level usability problems with an information system can be identified by this number of subjects [104] and [117]. Therefore, this was the target group size used for the evaluation of two GUIs.

There are a number of different options for study design and in the case of this thesis a "within-group" design was utilized for evaluating the GUIs. A within-group 
design involves individuals that are asked to try out different versions of a prototype system [104].

\subsection{Selection of Representative Experimental Tasks and Contexts}

The usability test was conducted in a controlled laboratory setting since it involved artificial conditions and tasks for the participants to complete. A written medical case description was required and served as stimulus material for the participants. A case description was created for each clinician type asking that individual participants complete a number of tasks while using the GUIs. The cases were developed with some input from clinicians on the research team in order to ensure they were "realistic and representative of real-life clinical situations and elicit high quality data about user interactions" [104]. The cases were specifically drawn from the type of situations commonly encountered during home care visits, which were identified in the previous section.

The goal was to make sure that the needs of all the primary end-users, the home care clinicians, could be addressed. The research conducted in sub-section 3.1 .3 was utilized at this point as it helped focus on the key user groups. Testing the prototypes and getting feedback from the user groups is the best way to understand whether the design ideas are providing them what they want from the system [109].

The following excerpt is the medical case description employed for the nursing usability tests:

You are about to arrive at your first client's house. The client, Joe Ranger, is an 80 year-old male who has mobility issues from a broken ankle. Six weeks ago, Mr. Ranger fell and broke his 
ankle at home. He had surgery in the hospital and has recovered well enough to go home. Although he is ambulating independently by using a walker, he is scared of falling again and therefore minimizing his activities, including the exercises that he was advised to do. He also states that he is still finds it painful to put weight on his affected ankle and shares that this ankle is affecting his sleep. He has been home now for 4 days.

You need to review Mr. Ranger's electronic health record in your car before you enter the house so that you can assess how Mr. Ranger's week went, whether or not there has been any improvement and how well he is coping with returning home from the hospital.

You access Mr. Ranger's file and need to:

- Find if he has been more sedentary this week

- Evaluate his activity patterns in the house

- Review the changes in his bed activity (e.g. his bed exit transfers)

- Identify and address reasons for the mobility issue

Examples of the medical case descriptions for the other home care clinician types used during the usability tests can be found in Appendix A. 


\subsection{Selection of the Evaluation Environment}

The next step of the evaluation was determining where the evaluation would take place. Six usability tests were conducted at the organizations or workplaces of the participants with the exception of two which were conducted off-site.

The location of the evaluations had to be flexible because of the hectic schedules of the participants and of the rare opportunity that the research team members were together in one location. As a result, the research team employed inexpensive and portable equipment that could be taken to actual work settings and set up appropriately for testing. For each usability test, the set-up involved a video recording of the computer screen - to document mouse movement and better understand the thought process and interface interaction of the participant - as well as a back-up audio recording.

Figure 4.1 illustrates the evaluation set-up with the participant (blue) and facilitator (red) seated at a table with the audio recorder positioned in front of the participant and the video recorder focused on the computer screen. Not shown in Figure 4.1 are the other members of the research team who were present as observers and for aid with any technical issues.

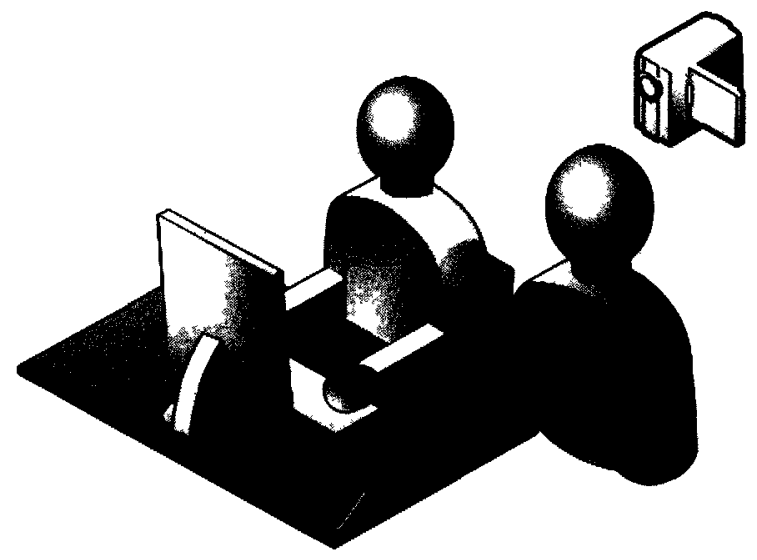

Figure 4.1: Evaluation set-up 


\subsection{Data Collection}

The research team conducted the usability testing with eight homecare clinicians (one occupational therapist, three nurses, and four case managers). Each session lasted approximately one hour and was documented by a full video recording of the computer screen with a concurrent audio taping of the participant-facilitator dialogue. The sessions were conducted with clinicians who had also participated in the previous project phase. The purpose of the testing was to solicit feedback from potential end-users about the specific design and application of the GUIs.

The interviews with the clinicians began by having them sign a consent form and being introduced to the research team. Each participant was informed by a facilitator about the project's intent and the purpose and approach of conducing usability tests. They were assured that there were no right or wrong answers and that the feedback they provided would help to improve the system by making appropriate changes.

The following script was provided to the facilitator for describing the project:

- A few months ago, the research team conducted interviews with home care providers about a proposed client monitoring system.

- The research team has compiled the information that was learned and is now ready to share some further developed ideas for your comment and feedback.

- The usability test is for the purpose of soliciting your opinion about the specific design and application of a client monitoring system.

- You will be presented with different 'screen shots' of the monitoring system, of what it could look like on a device such as a laptop, tablet computer or PDA. 
- You will be asked to express your opinion about the usefulness of the data presented for a client with mobility issues, the attractiveness of different features of the display (e.g. such as colour features, font size, and layout), and other desired functionality.

- There are no right or wrong answers. Your candid opinions are welcomed.

- The client monitoring system consists of a Bed Pressure Sensitive Mat installed inside the home, and a GPS/Accelerometer worn by clients during waking hours (likely embedded in a small cellular phone).

- It can be assumed that this information is transmitted wirelessly to a central computer server where it is stored and that this system works accurately, securely, and involves minimal burden on clients.

If the participants required more explanations regarding the technology used in the client monitoring system, the following scripts were prepared for the GPS and bed pressure sensor mat:

\section{GPS:}

- Imagine an application that could provide a summary of "what" a client has done and where, while taking into account various physiological sensors. For example:

o Time-in-home

o Time-out-of-home

- Time spent engaged in physical activity

○ Time spent idling

$\circ$ Heart rate over time

- Glucose-levels over time 
- Calories spent

\section{Bed Pressure Sensor Mat:}

- The bed pressure sensor mat is a waterproof sensor pad that is placed under a client's mattress

- The mat has 24 pressure sensors in a $3 \times 8$ grid configuration

- The mat occupies $24 \mathrm{~cm}$ by $90 \mathrm{~cm}$ with sensor elements spaced $10 \mathrm{~cm}$ apart

- As the bed-occupant moves, the pressure distribution on the sensor pad changes in proportion to the degree and type of movements made

- Software interprets this movement data to indicate the client's presence and posture while in bed

- This data can determine such parameters as: time-in-bed, length of time it took for a client to exit the bed, how symmetrical an exit was, and whether or not they used their hands to get out of bed

- Recording changes in bed entry and exit patterns may signify changes in the physiological condition of the client

Once the participant was comfortable with the purpose and description about the project, the facilitator explained the process of the usability test. The subsequent script was created for explaining the process to the participant:

- A usability test is a technique used to evaluate a product by testing it on users and helps researchers measure the ease of use of their product

- Today, we will ask you to read a scenario about a client of yours who has mobility issues 
- In the scenario there are some tasks that we would like you to try and accomplish using the prototype display

- During the time that you are interacting with the displays, we would like you to "think-aloud". This means we would like you to say whatever you are looking at, thinking, doing, and feeling, as you complete each one of the tasks outlined in the scenario.

- The feedback you provide us will help to make further improvements to the display.

- *Facilitator: demonstrate the "think aloud" method

- Example prompts for the facilitator during the usability test: "Please, keep on talking", "What are you thinking about now?", "What are you trying to do?", "Do you understand what is happening now?", "What happened?", "What do you expect to happen?", "Tell us what you are doing", “What do you think you are doing now?", "What do you understand happened?", can also try echoing the participant to get them to explain their comment

- Facilitator: if a participant asks a question concerning the design of the display, data, etc. try and divert answering them directly and instead ask a question back to get them to explain their thoughts more (e.g. Participant: "Why did you pick this colour scheme for the display?", You: "What don't you like about this colour scheme?", "Are there other colours that you would like to see?", "Is there something that is confusing you about the choice of colours?", etc.)

Using the case descriptions generated in section 4.3 , the clinicians were asked to complete specific tasks with two prototype interfaces. They were asked to 'think aloud' 
as they went through the case with each prototype so that their opinions about the usefulness of the data presented, the attractiveness of different features of the display, and other desired functionality could be captured on audio tape. This think aloud method is "one of the most useful techniques emerging from cognitive science" [104] and allows for qualitative data to be collected.

During the usability testing, the participants were prompted by the facilitator at key points in their interaction with the system to comment on aspects of the system and its design. Participants were encouraged to answer more specific questions such as:

- What other types of information would be helpful in assisting you with this caregiving scenario?

- What features / functions do you prefer?

- How could a feature / function be improved to meet your care-giving needs / tasks?

- Other types of displays / features / functions would you like and for whom?

The participants evaluated two versions of a prototype user interface that encompassed the data outputs of the technologies at Carleton and Wilfrid Laurier Universities. Each GUI version consists of three levels of data in a 'dig-down' format each subsequent level of data being more detailed than the previous. Figure 4.2 illustrates a schematic diagram of the approach to collecting video and audio recordings of the user interactions with the prototypes. 


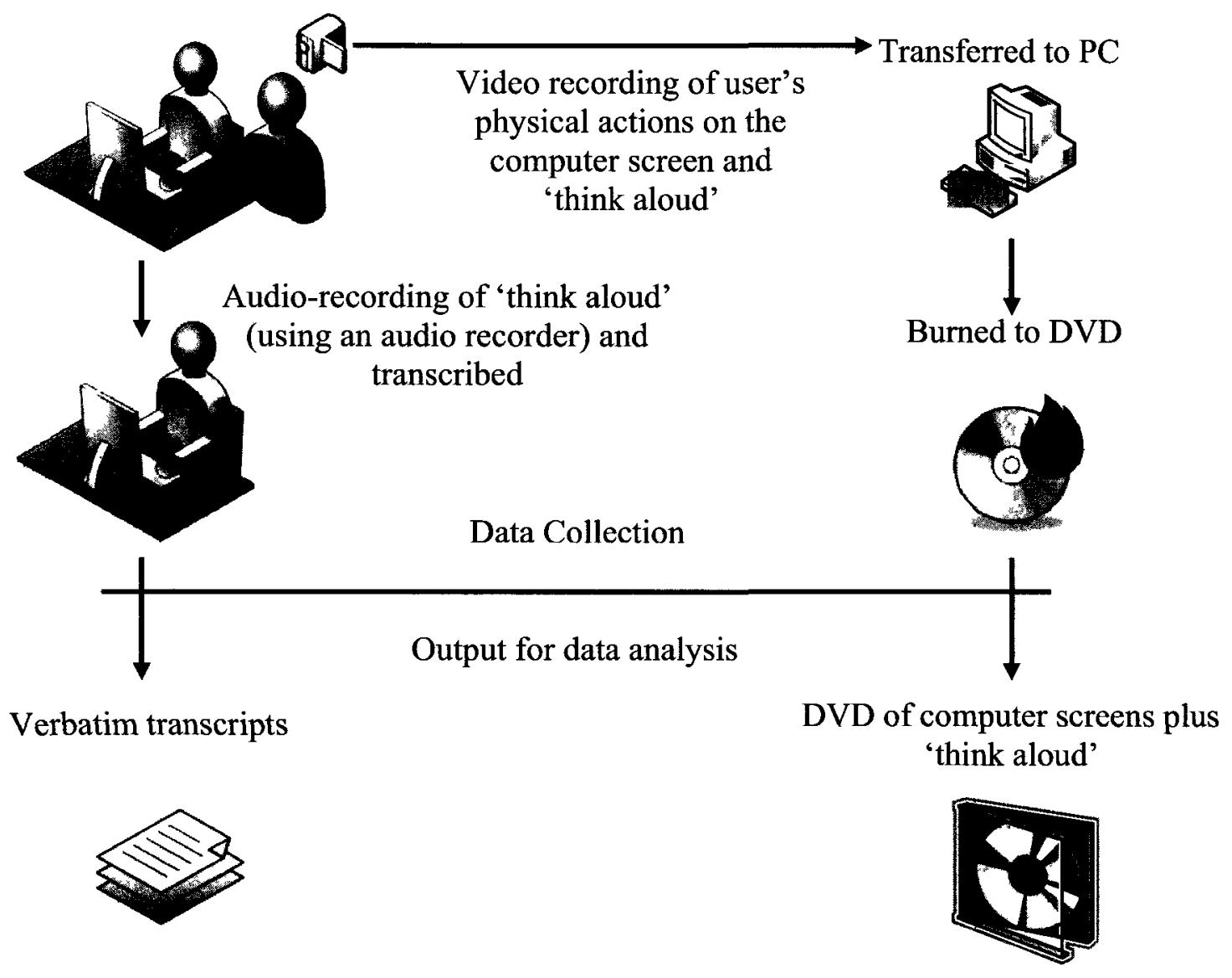

Figure 4.2: Video and audio based usability testing 


\subsection{Analysis of the Process Data}

The audio recordings were transcribed and used together with the video-recordings in the usability analysis. The video-recordings also had an audio overlay of the participants 'thinking aloud'.

Video recordings of participants interacting with the system are a great advantage because it provides "a record of the 'whole event"' [104].

Through iterative passes of the eight transcripts and videos, similar parameters surfaced. These parameters were listed in a table and grouped into two categories: issues pertaining to detailed design and issues related to conceptual design. Examples of detailed design issues include: font size and style, colours, graph axis locations, etc. Examples of conceptual design issues include: items that were not intuitive, navigation issues, how the information was grouped, learning time, etc.

Establishing these categories and extensively discussing their meanings allowed for greater inter-rater reliability in coding and completing the table. Space for other comments in each category was also included in the table in order to capture other themes or comments during the analysis and permit the opportunity of other common parameters to be established. As the videos became available, more iterative passes were completed along with the transcripts in order to note time stamps of the comments as well as any issues that were initially missed.

Table 4.1 displays the coding categories of the parameters as applied by Kushniruk and Patel [104] which focus on classical aspects of human-computer interaction. Table 4.2 displays the final analysis parameters and their descriptions. 
Table 4.1: Coding categories for qualitative analysis parameters [104]

\begin{tabular}{|c|c|c|}
\hline & I Coding critegories & F, Comment regarding: \\
\hline 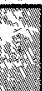 & Information Content & $\begin{array}{l}\text { Whether the information system provides too } \\
\text { much information, too little, etc. }\end{array}$ \\
\hline & Comprehensiveness of graphics and text & $\begin{array}{l}\text { Whether a computer display is understandable } \\
\text { to the subject or not }\end{array}$ \\
\hline 3 & Problems in Navigation & $\begin{array}{l}\text { Does the subject have difficulty in finding } \\
\text { desired information or computer screen? }\end{array}$ \\
\hline & Overall system understandability & $\begin{array}{l}\text { Understandability of icons, required computer } \\
\text { operations and system messages }\end{array}$ \\
\hline
\end{tabular}


Table 4.2: Qualitative analysis parameters

\begin{tabular}{|c|c|c|}
\hline \multicolumn{2}{|c|}{ Detailed Design Issues } & \multirow{2}{*}{$\begin{array}{l}\text { Comments regarding: } \\
\text { Trends on the homepage }\end{array}$} \\
\hline 新 & Version 'A' - Homepage Sparklines & \\
\hline 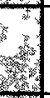 & Version 'A' - Homepage Bullet Graphs & $\begin{array}{l}\text { Averages on the homepage,, 'vertical' or } \\
\text { 'horizontal' lines, targets, etc. }\end{array}$ \\
\hline (3) & Would like more colours (other than B/W/R) & Additional colours \\
\hline 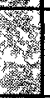 & $\mathrm{Y}$-axis description location & $\begin{array}{l}\text { Placement of units in the titles of the graphs } \\
\text { and not beside the graph axis }\end{array}$ \\
\hline the & Graph legends' location & $\begin{array}{l}\text { Placement of legends (e.g. Legends } \\
\text { underneath graphs indicating "day" and } \\
\text { "night" colour scheme, legend for 'personal } \\
\text { target' and 'requires attention' on homepage) }\end{array}$ \\
\hline tit & Preference of bar or line graphs & $\begin{array}{l}\text { (E.g. Line graph b/c "It's easier to see } \\
\text { the...pattern".) }\end{array}$ \\
\hline & Other comments & \\
\hline \multicolumn{2}{|c|}{ Conceptual Design Issues } & Comments regarding: \\
\hline 2 & Homepage preferred & (E.g. "I like the other one better") \\
\hline 4. & Version ' $\mathrm{B}$ ' homepage understandability & $\begin{array}{l}\text { Shape meaning, symbols in the middle of the } \\
\text { graphs, information they provide, etc. }\end{array}$ \\
\hline$=-1$ & Navigation & $\begin{array}{l}\text { Problems / ease of navigating through the } \\
\text { pages }\end{array}$ \\
\hline (2) & Liked \# of graphs on L1 & $\begin{array}{l}\text { Too much/little information, (not) crowded, } \\
\text { (not) cluttered, (specify if for Version 'A' or } \\
\text { Version 'B') }\end{array}$ \\
\hline the & Liked \# of graphs on L2 & $\begin{array}{l}\text { Too much/little information, (not) crowded, } \\
\text { (not) cluttered, etc. }\end{array}$ \\
\hline 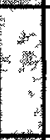 & Found red alerts useful & $\begin{array}{l}\text { Obvious, helpful, understandable (e.g. Found } \\
\text { red alerts a must and a "kind of universal } \\
\text { indicator") }\end{array}$ \\
\hline & Found targets on graphs useful & Obvious, helpful, understandable \\
\hline & Ability to view weekly/monthly/daily data & $\begin{array}{l}\text { Viewing trends over time, detail of } \\
\text { information, correlations between parameters }\end{array}$ \\
\hline 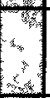 & Would use as a teaching tool & $\begin{array}{l}\text { Education, showing and involving clients in } \\
\text { their care plan }\end{array}$ \\
\hline the & Would like more info/ data on graphs & $\begin{array}{l}\text { Needing more information to make a } \\
\text { diagnosis, more targets, etc. (e.g. More } \\
\text { information via pop-up bubbles) }\end{array}$ \\
\hline+4 & Would like more numeric data & More numbers, less graphs, etc. \\
\hline 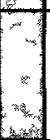 & $\begin{array}{l}\text { Preference of personal or general population } \\
\text { targets }\end{array}$ & $\begin{array}{l}\text { Ability to compare client to targets set from } \\
\text { population statistics or from the health care } \\
\text { provider }\end{array}$ \\
\hline 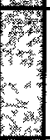 & Found data sets useful & $\begin{array}{l}\text { Satisfaction with information provided, } \\
\text { useful information to deduce a plan of care, } \\
\text { etc. }\end{array}$ \\
\hline in & Would like a warning trend color & $\begin{array}{l}\text { Additional colours to help distinguish } \\
\text { severity of a client's data }\end{array}$ \\
\hline
\end{tabular}




\begin{tabular}{|l|l|l|}
\hline \multicolumn{2}{|l|}{ Conceptual Design Issues } & Comments regarding: \\
\hline & Ability to explode graphs & Size, legibility, etc. \\
\hline & Would like to customize the display & $\begin{array}{l}\text { Generating data sets, comparing or showing } \\
\text { only certain data, etc. }\end{array}$ \\
\hline & Would like the ability to set targets & Customizing targets to each client \\
\hline & Other comments & \\
\hline
\end{tabular}

$\mathrm{L} 1=$ homepage

$\mathrm{L} 2$ = detailed information of each parameter accessed directly from the homepage or when one of the major tabs at the top of the display are selected

\subsection{Interpretation of Findings}

The table constructed from the analysis of the videos and transcriptions is a summary of user preference data. This type of summary was chosen because there was:

1) No timing of the completion of the task due to the 'think aloud' method;

2) Task accuracy was not calculated, and;

3) Frequency and classes of problems encountered were not counted.

The following sub-sections provide example screenshots outlining some of the issues discovered along with the corresponding parameter and the interpretation of their findings.

\subsubsection{Detailed Design Issues}

Parameters: "Y-axis description location" and "Graph legend's location"

Figure 4.3 is an example of the manner in which detailed weekly information of each parameter is shown (the particular parameter shown indicates the number of hours a home care client has been in bed during the day and night). 


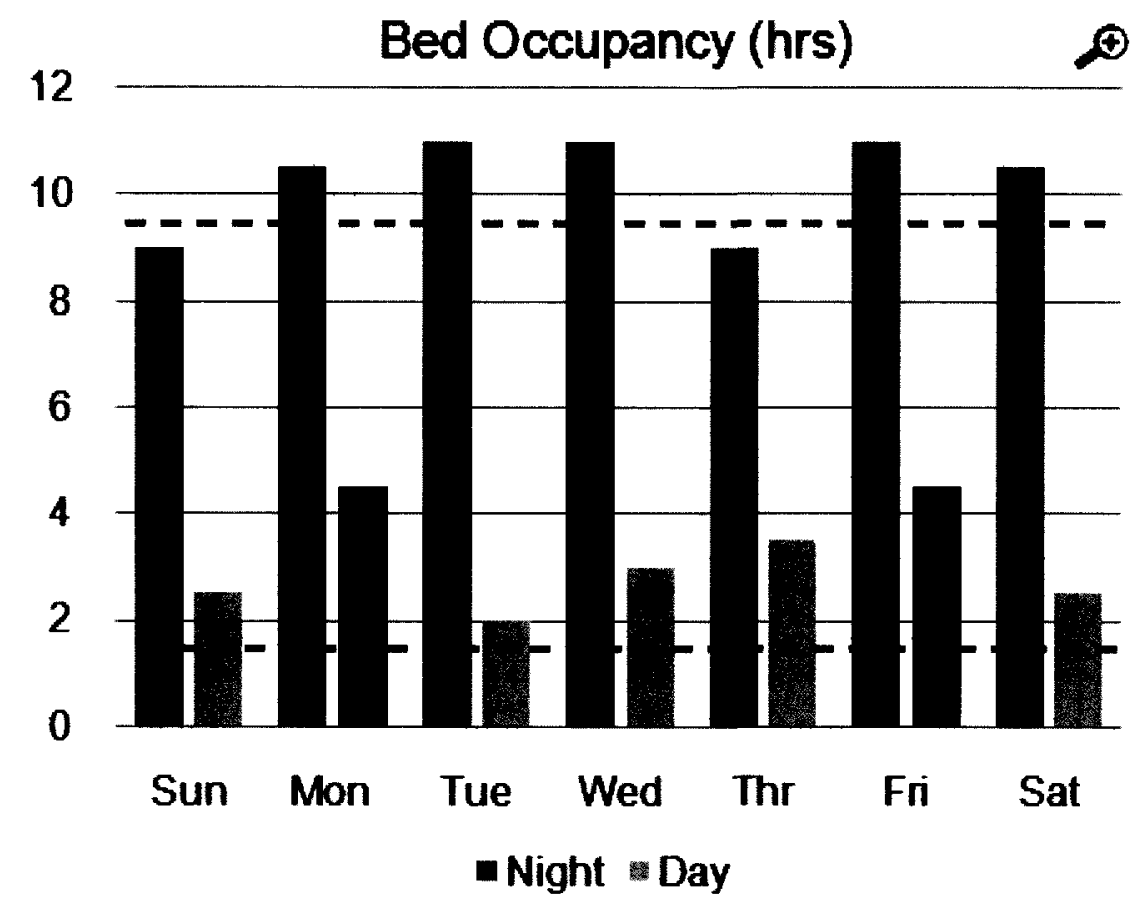

Figure 4.3: Representation of detailed weekly information of parameters

The user can access this type of information when they have selected a parameter directly from the homepage or when they select one of the major tabs at the top of the display. The major tabs include the following: home, client information, bed activity, outdoor activity, and inside activity (Figure 4.4 and Figure 4.5).

Three participants found the location of the $y$-axis title confusing and would prefer to have it beside the axis instead of in the graph's title. Four participants also felt that the graph legends had to be closer to the graphs that they belonged to. Additionally, a participant expressed that the homepage legend for Version ' $A$ ' and for Version ' $B$ ' had to be more obvious; suggesting larger font size (Figure 4.4 and Figure 4.5). 


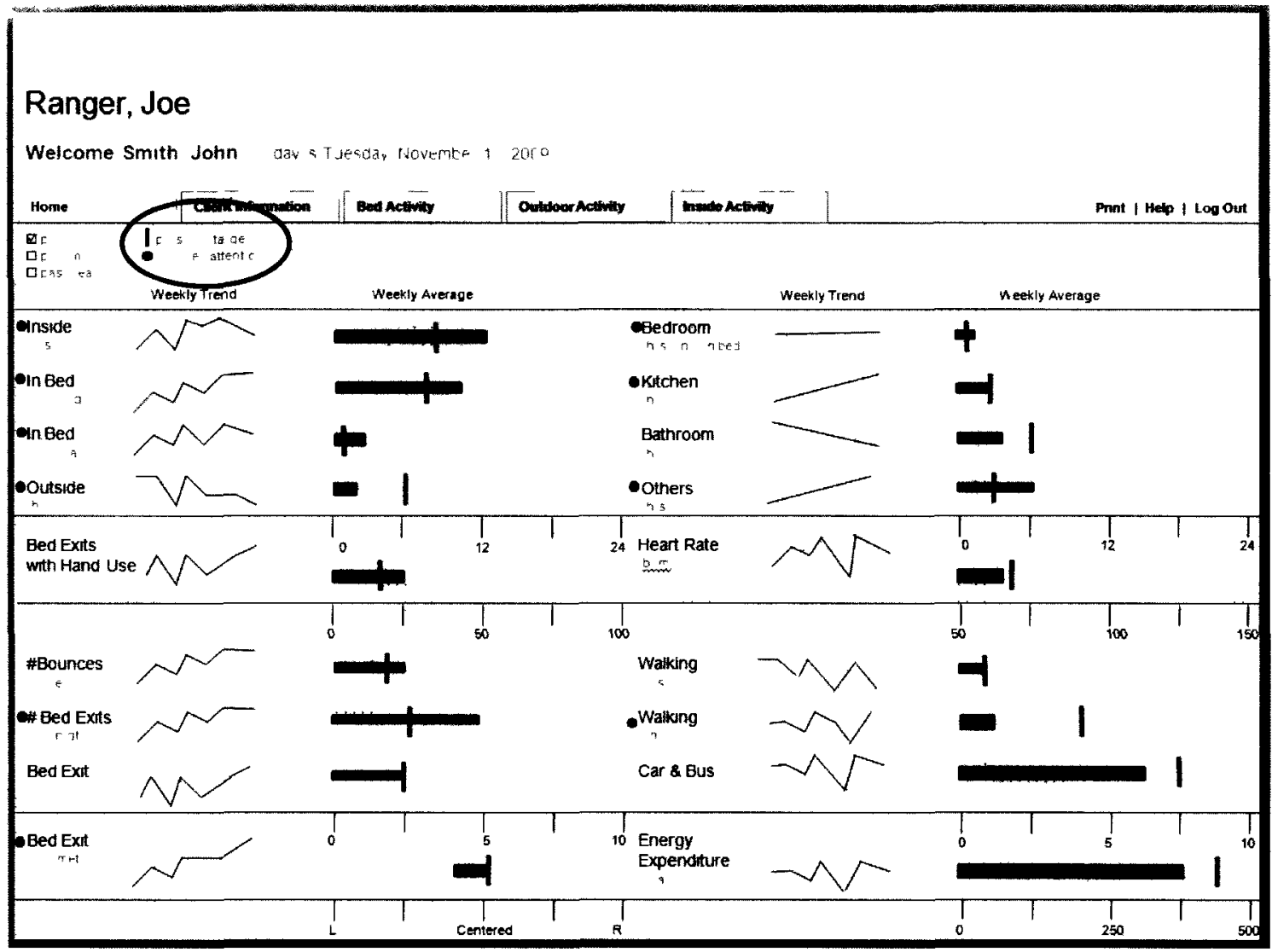

Figure 4.4: Legend location and size for the homepage on Version ' $A$ ' 


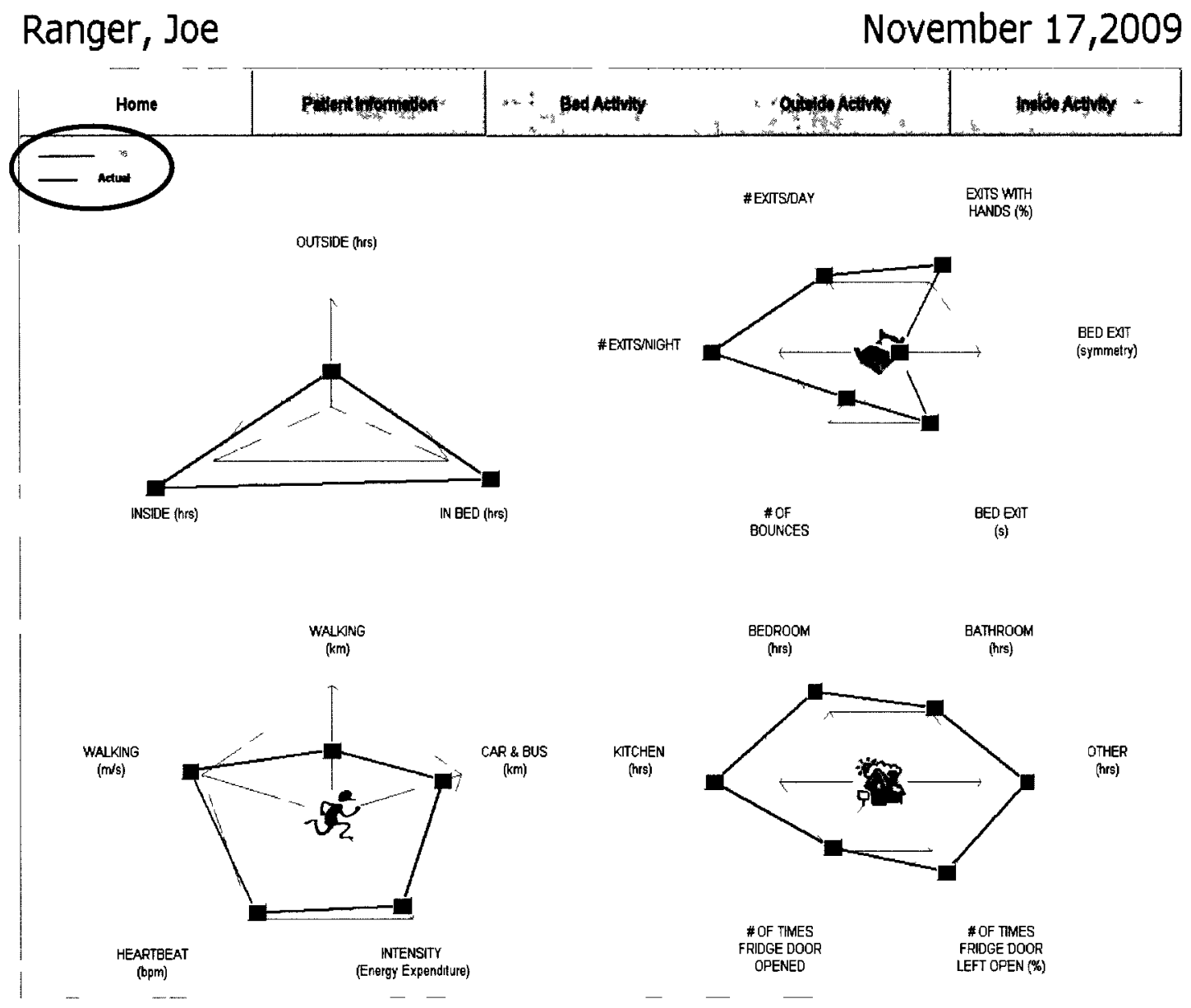

Figure 4.5: Legend location and size for the homepage on Version 'B'

There are a few other detailed design issues worth mentioning:

- Four participants would have liked to see more use of colours other than black, white, and red

- Six participants preferred bar graphs to line graphs

- One participant would have liked a number to appear as they scrolled over data points on the graphs

- One participant thought that something flashing would also help to indicate an alert for the client's health 


\subsubsection{Conceptual Design Issues}

Parameters: "Version 'A' -Homepage Sparklines" and "Version 'A' - Homepage Bullet Graphs"

Five participants found the sparklines to be confusing and overwhelming information for the homepage (Figure 3.7). Some found them distracting and made the screen too crowded. For example, one participant's comment regarding the sparkline was "...the "Weekly Trend", I don't get that...so can you explain to me a little about what [the sparklines] mean?" (Participant 3, 2:10, 56:31 3, 25).

The bullet graphs were better received as three participants easily understood them, immediately understood that the graphs indicated an average, and that the vertical bars "means that it's some kind of limitation" (Participant 5, 3:19-8:42, 4-6).

Parameters: "Liked \# of graphs on L1" and "Liked \# of graphs on L2"

The overall consensus of the number of graphs both on the homepage, L1, and within any of the major tabs, L2, was acceptable (Figure 4.4 and Figure 4.5 for L1 and Figure 4.6 and Figure 4.7 for L2). At the beginning of the tests, most participants were overwhelmed by the number of graphs but as they started using the prototypes, they became more comfortable with the amount of information displayed. 


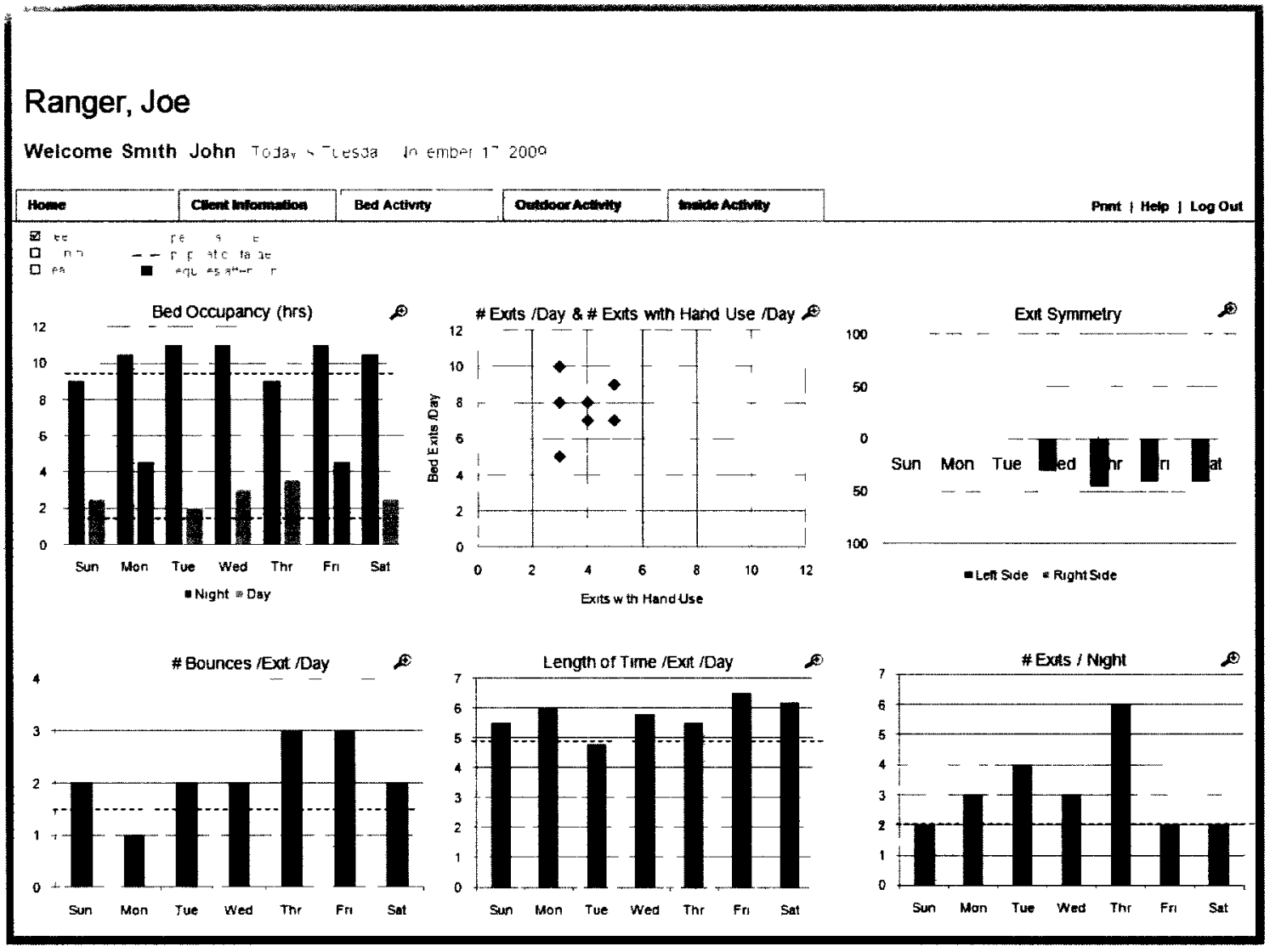

Figure 4.6: Bed activity tab in Version ' $A$ ' 


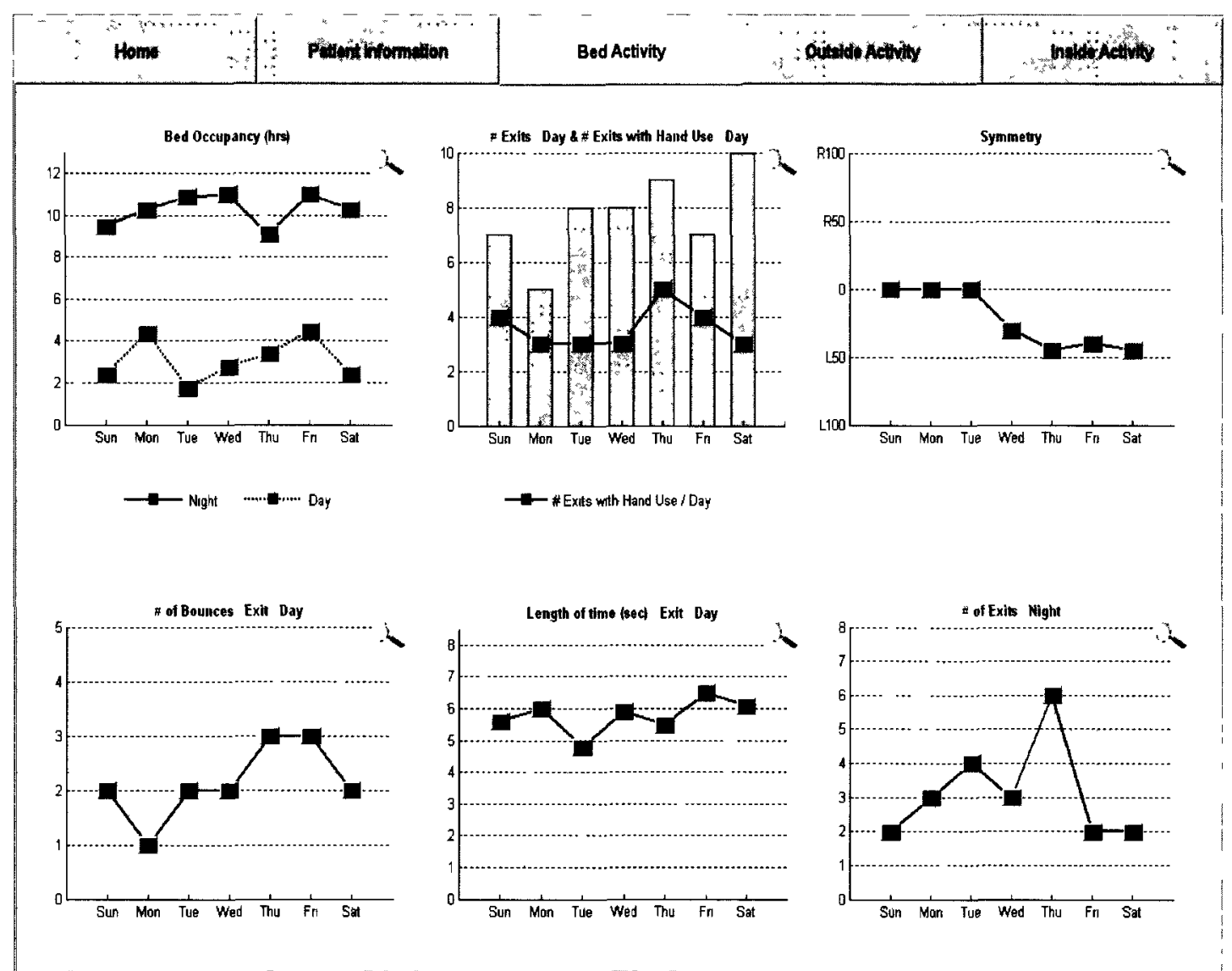

Figure 4.7: Bed activity tab in Version ' $B$ '

Parameter: "Found red alerts useful"

Red was used on the homepage either beside the parameter name (Figure 4.4) or as the data point (Figure 4.5). It was used to indicate abnormal data also in L2 as a red bar (Figure 4.6) or as a point (Figure 4.7).

The use of red as an indicator for "requires attention" was found to be helpful. Six participants found that red alerts were obvious and "a must...a kind of universal indicator" (Participant 1, 9:18, 20:51, 6, 10) for an alert.

Parameters: "Found targets on graphs useful", "Preference of personal or general population targets" and "Would like the ability to set the targets" 
Indicators for the client's personal target for all the parameters were used within both versions of the display. On the homepages, targets were displayed as bullet graphs or as polar stars (Figure 4.8).

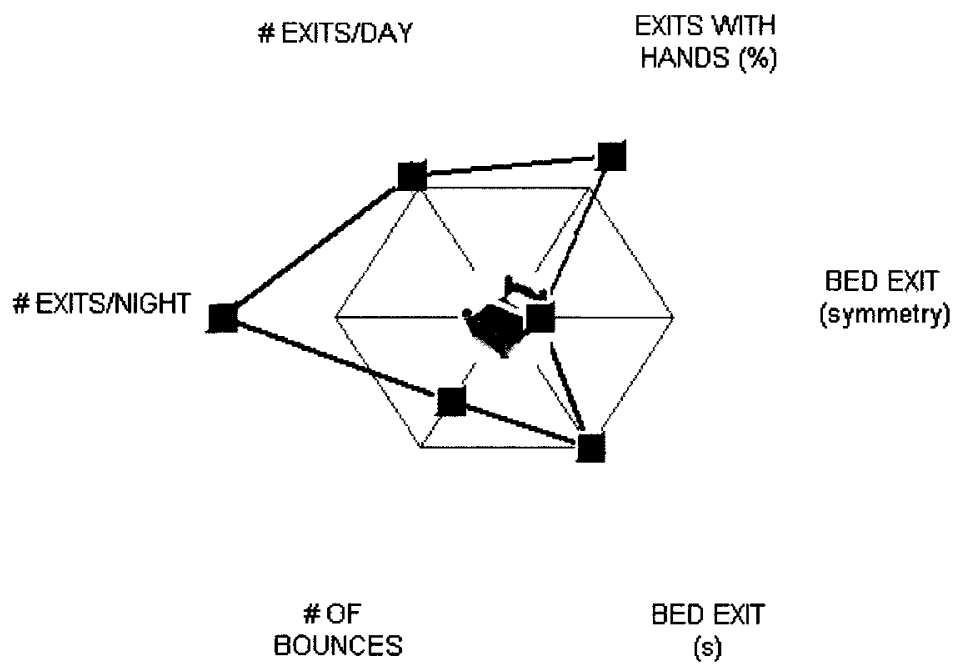

Figure 4.8: Example of client target in $\mathrm{L1}$ for Version 'B'

Targets for L2 were shown as either a dashed or solid horizontal line across line or bar graphs (Figure 4.9).
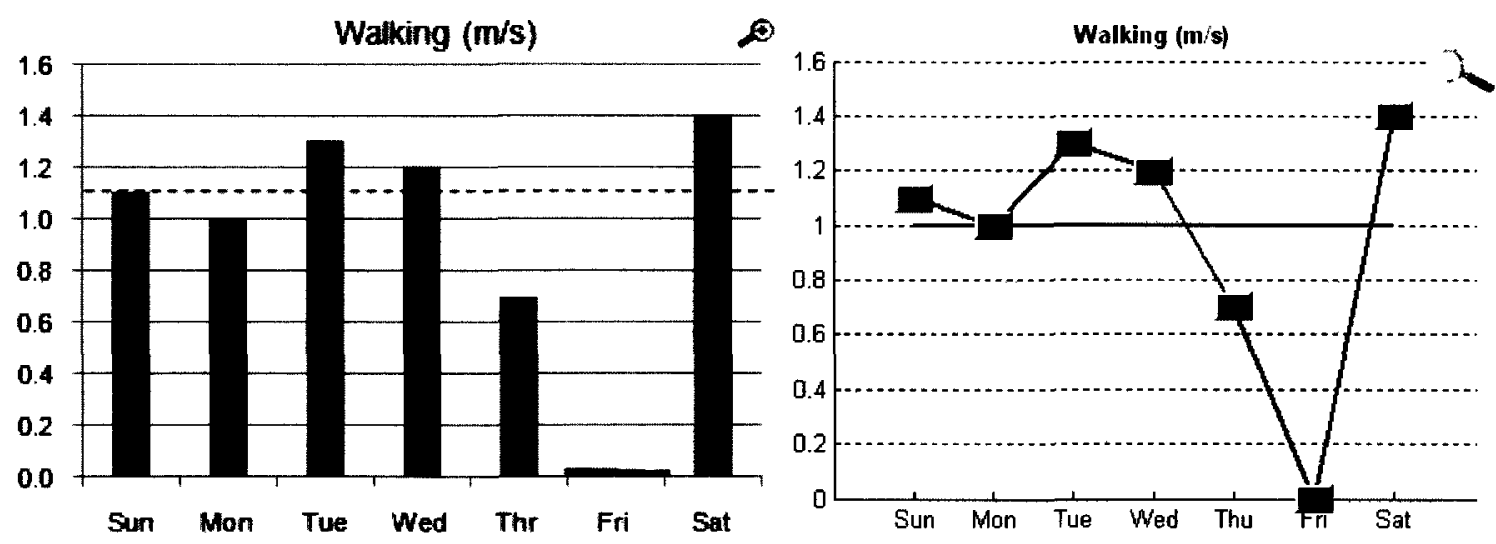

A)

B)

Figure 4.9: Example of client target at $L 2$ for $A)$ Version ' $A$ ' and B) Version 'B' 
Five participants felt that these targets were useful for them in providing care to the client. They also preferred that the target be a personal target set by them for each individual client. For example, "I like personal [targets] because everybody is different" (Participant 6, 20:03, 9). Another participant contributed:

Yeah, and I'll tell you why because a lot of the cognitive testing that I do it's based on the average population it's been tested for and you know it's kind of hard to make generalizations because everyone's personal situation is so different that sometimes I skew from what the population norm is saying because you know what's happening to this client in this part of town in this economic and social situation is absolutely having an effect on their score as opposed to this been tested on and it's good to have that comparison, but again I'm just very sensitive about that because it is a very personal matter (Participant 4, 33:40, 17).

Parameter: "Ability to explode graphs"

If a user accesses the data sets in the major tabs at the top of the display such as the bed, outdoor, or inside activity tab, they have the ability to 'explode' any of the available graphs within that tab. At the top of each graph is a magnifying glass (Figure 4.10) and when this magnifying glass is selected, the corresponding graph enlarges. Four participants found this feature very helpful. 


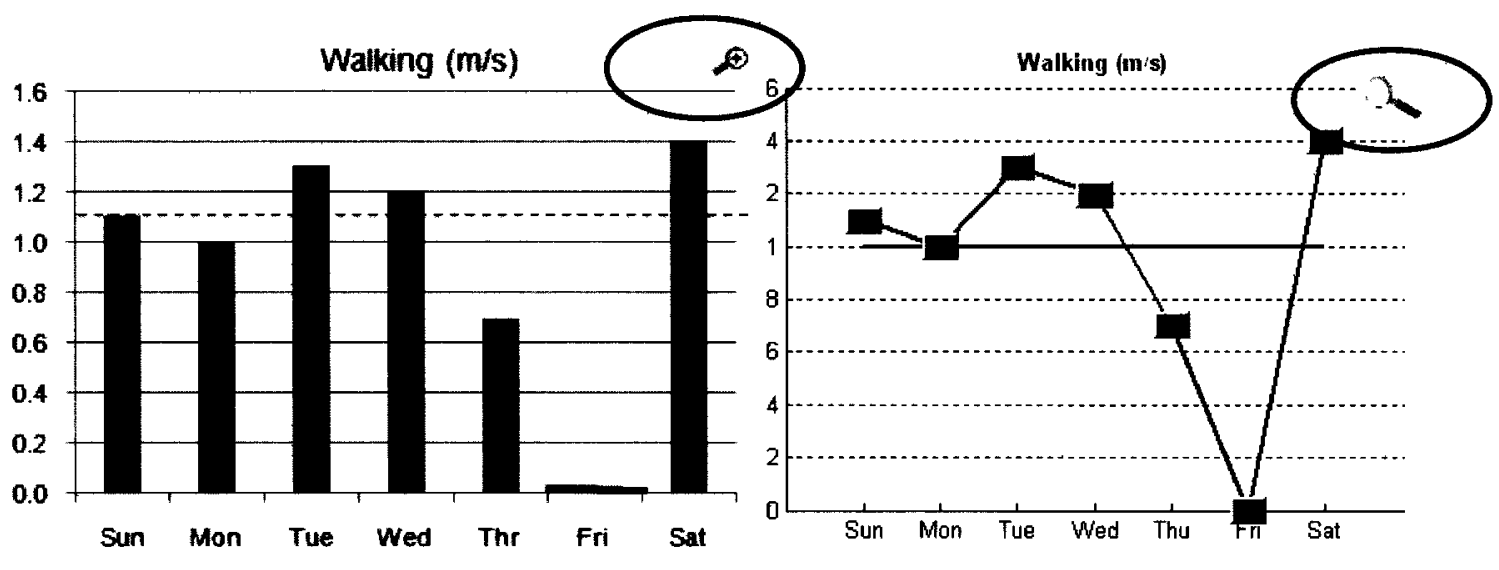

A)

B)

Figure 4.10: Example of magnifying glass at L2 for A) Version ' $A$ ' and B) Version ' $B$ '

For example, one participant stated "yes, because I can see from [the screen] on Wednesday both of [the data points] are red and like what was going on with your heart rate, that's pretty significant and then I can pop that up and see" (Participant 4, 41:04, 20). Another participant commented that the magnifying glass was "kinda cool. And then any of these graphs you can just get them bigger" (Participant 7, 9:00, 5).

There are a few other conceptual design issues worth mentioning:

- Participants navigated with ease

- Four participants liked the ability to view weekly and daily data on the prototype and would like the ability to change the time period to monthly as well (this feature was not possible to incorporate for the testing due to time constraints and was simply indicated as a place holder on the homepage)

- Three participants indicated they would use the display as a teaching tool during client visits 
- Three participants would have like graphs, tabs, etc. to have definitions. For example, "let's say if [they] put [their] cursor on the word 'inside"" a "pop-up or a um, the definition of let's say 'inside"” would appear (Participant 1, 7:42, 5)

- Four participants stated that they do not feel like they need any more numerical data

- Four participants would like a warning trend colour (i.e. not only the red indicator that something requires attention)

- One participant liked that they could "scroll through the future and into the past" (Participant 1, 33:50, 12) with the detailed parameters information.

- One participant mentioned that they would be able to provide better care with the application and that the trends are useful information to have in the system (Participant 1, 35:49, 16)

\subsubsection{Evaluation Outcomes}

The following table (Table 4.3) outlines the overall success of the system as related to the usability goals presented in sub-section 3.3.1. 
Table 4.3: Success of system as related to usability goals

\begin{tabular}{|c|c|c|}
\hline $\begin{array}{l}\text { Interaction } \\
\text { and Outcome } \\
\text { Criteria }\end{array}$ & Usability Goals & Success of System Meeting Requirements \\
\hline Learning & $\begin{array}{l}\text { Minimum learning } \\
\text { required }\end{array}$ & $\begin{array}{l}\text { Most participants were able to navigate easily } \\
\text { through the interfaces. They felt that if they were } \\
\text { to use the application "all the time to interpret it } \\
\text { then [they would] know exactly where to hone to } \\
\text { zone in to" for the location of certain items/ } \\
\text { legends/ graphs, etc. (Participant } 1,3: 12,4 \text { ). }\end{array}$ \\
\hline Efficiency & $\begin{array}{l}\text { Minimum number of } \\
\text { actions }\end{array}$ & $\begin{array}{l}\text { Presenting all the data sets that the sensors could } \\
\text { collect at the front end helped to minimize the } \\
\text { number of actions }\end{array}$ \\
\hline Efficiency & $\begin{array}{l}\text { Should be able to work fast } \\
\text { - provide shortcuts }\end{array}$ & $\begin{array}{l}\text { The homepages on both versions allowed the } \\
\text { clinicians to select data sets they were interested in } \\
\text { for more detailed information without going to one } \\
\text { of the major tabs }\end{array}$ \\
\hline $\begin{array}{l}\text { Reduce } \\
\text { cognitive load }\end{array}$ & $\begin{array}{l}\text { Users should not have to } \\
\text { remember anything }\end{array}$ & $\begin{array}{l}\text { No data sets were needed to be remembered from } \\
\text { screen to screen }\end{array}$ \\
\hline $\begin{array}{l}\text { Reduce } \\
\text { cognitive load }\end{array}$ & $\begin{array}{l}\text { Direct manipulation: } \\
\text { visible objects, visible } \\
\text { choices }\end{array}$ & $\begin{array}{l}\text { Participants would like to be able to customize the } \\
\text { information to show only the relevant data sets for } \\
\text { their profession and client. For example, "it's a lot } \\
\text { of information to just go in and go through" } \\
\text { (Participant } 7,8: 32,5 \text { ) and another participant } \\
\text { mentioned that "like some reports when you want } \\
\text { to go in depth you can just click and click and then } \\
\text { it generates it own different types of reports, } \\
\text { however you wanted it. Whichever is easier for } \\
\text { you to read" (Participant } 1,10: 58,6 \text { ). }\end{array}$ \\
\hline $\begin{array}{l}\text { Error } \\
\text { prevention; } \\
\text { learning }\end{array}$ & $\begin{array}{l}\text { Clear display of } \\
\text { information }\end{array}$ & $\begin{array}{l}\text { Some participants wanted larger font and more } \\
\text { obvious positions for graph legends and y-axis } \\
\text { titles located beside the graph instead of in the } \\
\text { graph's title }\end{array}$ \\
\hline $\begin{array}{l}\text { Error } \\
\text { reduction and } \\
\text { prevention }\end{array}$ & $\begin{array}{l}\text { Use simple, natural dialog; } \\
\text { eliminate extraneous words } \\
\text { / graphics }\end{array}$ & $\begin{array}{l}\text { Dialog for some participants was unfamiliar and } \\
\text { therefore, options for explanations/ definitions } \\
\text { would be needed in the next version }\end{array}$ \\
\hline Experience & $\begin{array}{l}\text { Should function easily to } \\
\text { reduce any high frustration } \\
\text { level which would degrade } \\
\text { the user performance and } \\
\text { be aiding and not } \\
\text { distracting from the job at } \\
\text { hand }\end{array}$ & $\begin{array}{l}\text { Some participants felt overwhelmed by the } \\
\text { appearance and thought the screens were "too } \\
\text { confusing...too overcrowded" (Participant } 8 \text { ). } \\
\text { Therefore, the GUI needs to be redesigned and } \\
\text { simplified so not to distract or degrade } \\
\text { performance. }\end{array}$ \\
\hline
\end{tabular}

\subsection{Iterative Input into Design}

Implementing changes to the prototype interfaces based on the recommendations in the

previous section will help improve the success of the system. Since this was a formative 
evaluation, additional evaluations can be repeated to reveal how changes made to the prototype affect the system's usability. This method helps to integrate the feedback from the users in the design and development process in order to continuously improve the information system and help gauge/measure any developmental progress. 


\section{Chapter 5}

\section{Data Mining Techniques for Home Care Client Health}

\section{Data}

This chapter presents an example medical condition where features from home care client's data must be detected and two different types of data mining techniques which can be applied. The first data mining technique uses a segmentation algorithm that splits a client's data into linear segments and the second technique uses a neural network. The two techniques were developed and evaluated to determine which was best suited for varying levels of noise and for varying data trend types. Their performance was also assessed to reveal the best method for establishing values for the parameters of each technique.

\subsection{Example Clinical Cases for Frequent Night-time Bed Exits}

Home care clinicians who participated in the focus groups (see sub-section 3.2.3) noted that one of the client groups for whom it would be beneficial to have remote monitoring technologies, such as a bed pressure sensitive mat, were those with incontinence issues. Incontinence issues are caused by a number of clinical conditions which result in a client 
needing to exit their bed often during the night. A few examples of such conditions include benign prostatic hyperplasia, urinary tract infection, nocturia, and diabetes.

In order to understand the clinical significance of capturing and monitoring a parameter such as the number of bed exits during the night, a review of benign prostatic hyperplasia (BPH) is presented. $\mathrm{BPH}$ is a "non-cancerous enlargement of the prostate gland...the most common benign tumour in aging men" [118], and a common condition for which male patients seek treatment [119]. This condition greatly diminishes the quality of life amongst men and is the most common cause of lower urinary tract symptoms and bladder outlet obstruction [120]. Improving the patient's urinary symptoms and the quality of life are some of the management goals of BPH.

BPH symptoms are caused by irritation or obstruction. Examples of irritation symptoms include frequency, urgency and nocturia. Examples of obstruction symptoms include a weakened urinary stream, hesitancy, and a need to push to initiate micturition (the act of urinating). BPH may also be caused by diabetes mellitus, Parkinson's disease, and stroke [119].

Current treatment of $\mathrm{BPH}$ is a transurethral resection of the prostate. There are significant complications of this procedure which include haemorrhage during or after the procedure which often calls for a blood transfusion, transurethral resection syndrome, urinary incontinence, bladder neck stricture, and sexual dysfunction. There are other treatments for BPH being explored that may eventually have the potential to rival transurethral resection of the prostate if their effectiveness is demonstrated in the long term [121]. 
There have been international studies conducted that demonstrate an elevated occurrence of BPH with increasing age. It has also been shown that in almost all elderly men, autopsy data indicates anatomical or microscopic evidence of BPH. Prevalence of BPH from autopsy studies of 1,075 subjects show that the condition is evident in $8 \%$ of men $30-39$ years, $40-50 \%$ of men $50-59$ years, and more than $80 \%$ in men over 80 years [122]. A Canadian telephone survey conducted by Norman et al. [123] found that overall, $23 \%$ of men 50 years of age or older experienced moderate to severe symptoms associated with BPH.

\subsection{Data for Mining Simulations}

\subsubsection{Data Features}

There are a number of features which may aid in clinical decision making regarding home care clients who have a condition that requires monitoring their number of bed exits. These crucial features and how they may be mined from the client's data will be discussed. For the purpose of the discussion, a few terms related to the client's data need to be identified.

Figure 5.1 shows what could be normal data gathered from a home care client regarding their number of bed exits per night. What defines a 'night' for one client may not be the same length of time as for another, and therefore, this would have to be determined for each individual client. 


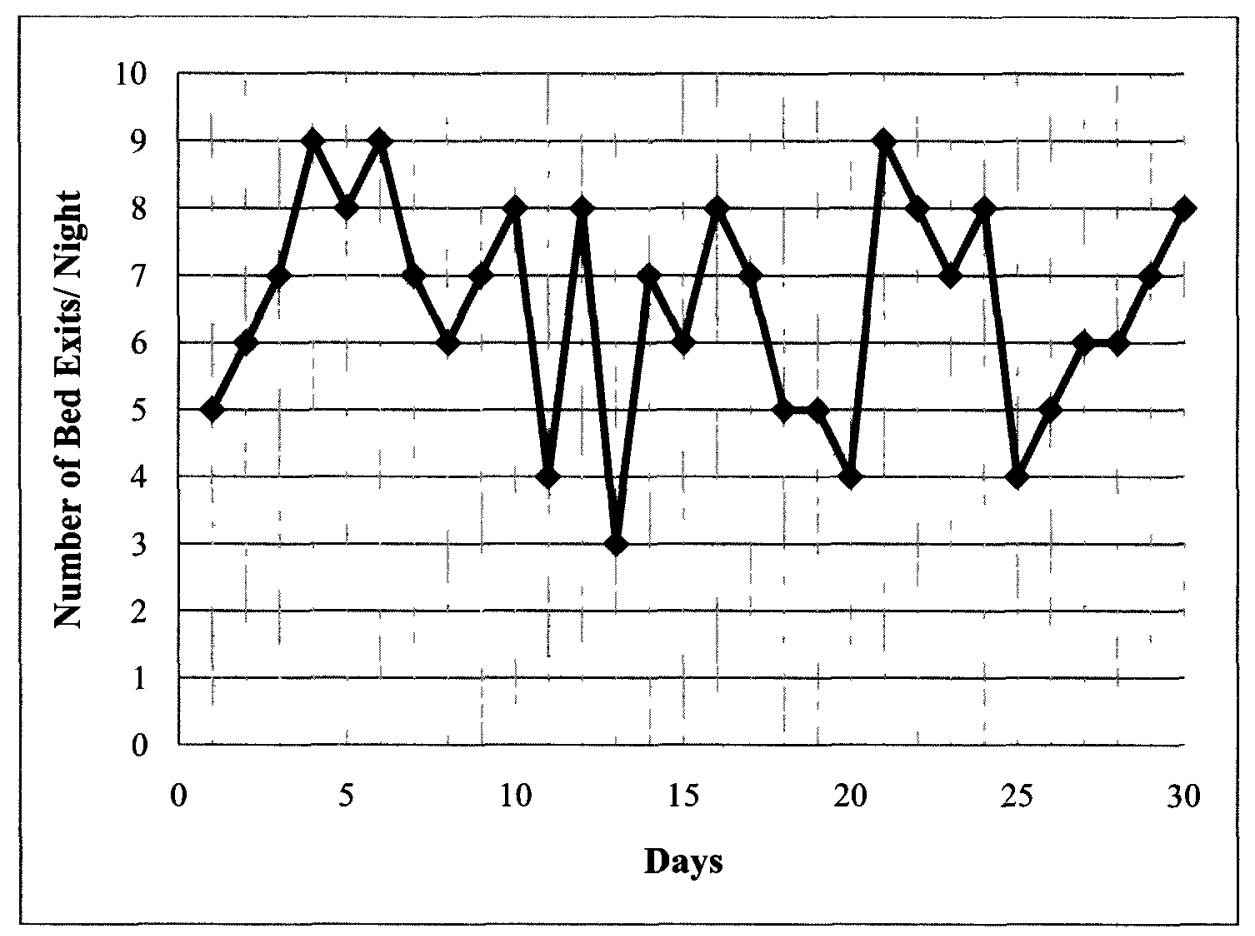

Figure 5.1: A simulated example of a home care client's bed exit data

Given this data, a 'normal' range of bed exits of the client can be detected by a simple algorithm identifying the minimum and maximum values. Related to these 'normal' minimum and maximum values, which can be referred to as lower and upper control limits, are hard tolerances [68]. Hard tolerances lie outside the control limits and for the purposes of home care client monitoring, can represent emergent critical situations. In the example case above, a clinician may set the lower and upper hard tolerances as 1 and 11 exits per night as those tolerances may represent an urgent situation. For a client's data, the average (mean) number of bed exits can also be detected. Figure 5.2 illustrates the parameters aforementioned which could be also be collected and output to a clinician on an interface. 


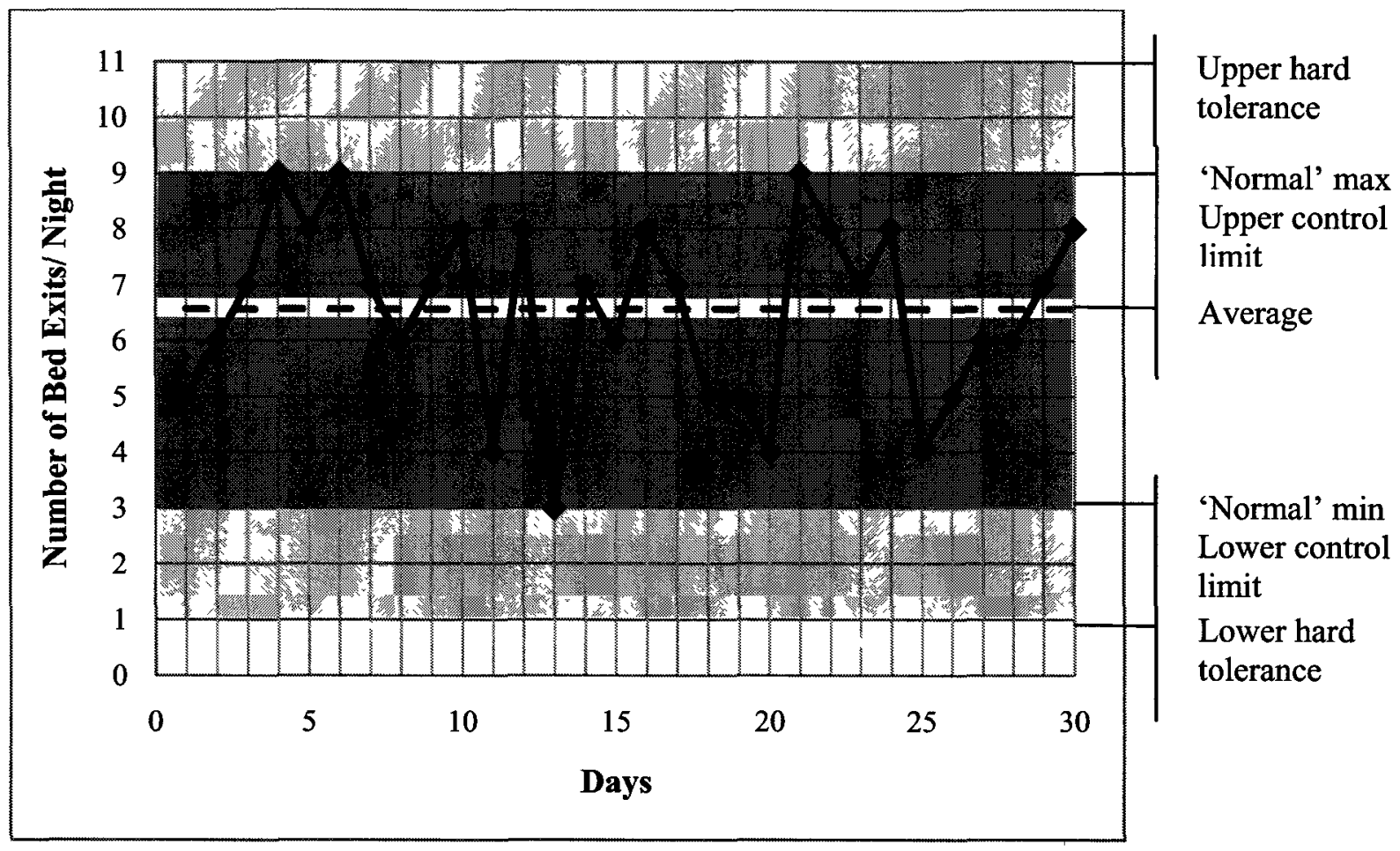

Figure 5.2: Average and 'normal' minimum and maximum ranges of a home care client's bed exit data

Outliers in a home care client's data may also be useful for a clinician to know in order to identify a clinical situation or problem. An 'outlier' is an observation that lies outside of the overall pattern of a distribution [124]. These inconsistent observations may be genuine observations that are of interest and could be the most valuable because they represent deviations from normality. Outlier values may also be errors caused by a number of factors and therefore, do not represent the data accurately. Furthermore, many data mining techniques are sensitive to outlying values and cannot tolerate them well and for all these reasons, they must be detected from a client's data [125].

An outlier is often very straightforward to identify and is a data point that falls more than 1.5 times the interquartile range (IQR), above the third quartile, or below the first quartile [124]. In some cases, the lower and upper outlier values may be a feature a 
clinician would like to set themselves for a particular home care client in order to alert them of a particular condition.

In the case of the example data, the IQR results in 3 bed exits, the third quartile as 8 , and the first quartile as 5 . Therefore, if data falls more than 1.5 times the IQR, above 8 , or below 5 it can be classified as an outlier. Another common method of detecting outliers is to look for data points more than a certain number of standard deviations, $\sigma$, from the mean, $\mu$. Depending on the request of a clinician, a simple algorithm can be used to capture outliers that are:

- A one instance outlier above or below 'normal' client's range

- A one instance outlier above or below a client's range set by a clinician

- Outliers above or below 'normal' client's range lasting $x$ days

- Outliers above or below a client's range lasting $x$ days set by a clinician There are however, a few disadvantage of this type of method for detecting outliers. Monitoring systems that are equipped with similar limit alarm systems for detecting outliers are prone to generating a large number of false alarms which become an extra burden for clinicians. These types of alarm system do not take into context temporal trends or pattern recognition on the monitored signal (steady state, level change, slow increase, etc.) and therefore, may not correspond to a correct physiological change [126].

Another feature which may be of interest for a specific home care client is data trends as identified by the home care clinicians in sub-section 3.2.3. Trends may detected by the numerous techniques introduced in sub-section 2.2 .2 such as by first-order primitives in which case they are extracted by the segmentation algorithm or by 
calculating the sign, \pm , of the linear function's slope that best fits the parameter on a fixed duration moving window [127] and [128].

Useful trends for a clinician may include:

- Increasing/ decreasing trend towards a target over $x$ days

- Increasing / decreasing trend away from a target over $x$ days

- Increasing/ decreasing trend over $x$ days

- Steady trend over $x$ days

As an example, a clinician may want to know if the client begins to get out of bed more often. Fitting a function through data, of which the clinician has no prior understanding, can be as simple as to fit a straight line. The most usual choice is leastsquares fit. If noise in the data is restricted to the y-coordinate (i.e. not affecting time on the x-coordinate), the least-squares fit minimizes the function (4) with respect to each $a_{j}$ [129].

$$
\begin{aligned}
& S\left(a_{0}, a_{1}, \ldots, a_{m}\right)=\sum_{i=0}^{n}\left[y_{i}-f\left(x_{i}\right)\right]^{2} \\
& \text { Where } f(x)=f\left(x ; a_{0}, a_{1}, \ldots, a_{m}\right)
\end{aligned}
$$

As a result, the optimal values of the parameters are given by the solution of the equation

$$
\frac{\partial S}{\partial a_{k}}=0, k=0,1, \ldots, m
$$

In (4), the terms $r_{i}=y_{i}-f\left(x_{i}\right)$ are called residuals. These residuals denote the discrepancy between the data points and the fitting function at $x_{i}$. Therefore, the function Sto be minimized is the sum of the squares of the residuals [129].

In fitting a straight line (6) to the example data illustrated above,

$$
f(x)=a+b x
$$


the function to be minimized is (7).

$$
S(a, b)=\sum_{i=0}^{n}\left[y_{i}-f\left(x_{i}\right)\right]^{2}=\sum_{i=o}^{n}\left(y_{i}-a-b x_{i}\right)^{2}
$$

Equation (5) now becomes

$$
\begin{aligned}
& \frac{\partial S}{\partial a}=\sum_{i=0}^{n}-2\left(y_{i}-a-b x_{i}\right)=2\left[a(n+1)+b \sum_{i=0}^{n} x_{i}-\sum_{i=0}^{n} y_{i}\right]=0 \\
& \frac{\partial S}{\partial b}=\sum_{i=0}^{n}-2\left(y_{i}-a-b x_{i}\right) x_{i}=2\left[a \sum_{i=0}^{n} x_{i}+b \sum_{i=0}^{n} x_{i}^{2}-\sum_{i=0}^{n} x_{i} y_{i}\right]=0
\end{aligned}
$$

Dividing both of the equations (8) and (9) by $2(n+1)$ and rearranging the terms, the mean values of the $x$ and $y$ data can be solved (10)

$$
\bar{x}=\frac{1}{n+1} \sum_{i=0}^{n} x_{i} \quad \bar{y}=\frac{1}{n+1} \sum_{i=0}^{n} y_{i}
$$

The solution for the parameters in (6) now becomes

$$
b=\frac{\sum y_{i}\left(x_{i}-\bar{x}\right)}{\sum x_{i}\left(x_{i}-\bar{x}\right)}, \quad a=\bar{y}-\bar{x} b
$$

Using the least-squares fit method described above on the example data, the following decreasing trend can be observed (Figure 5.3). This trend line could be a parameter that a clinician would like to know or be alerted of pending their requests for a particular client. 


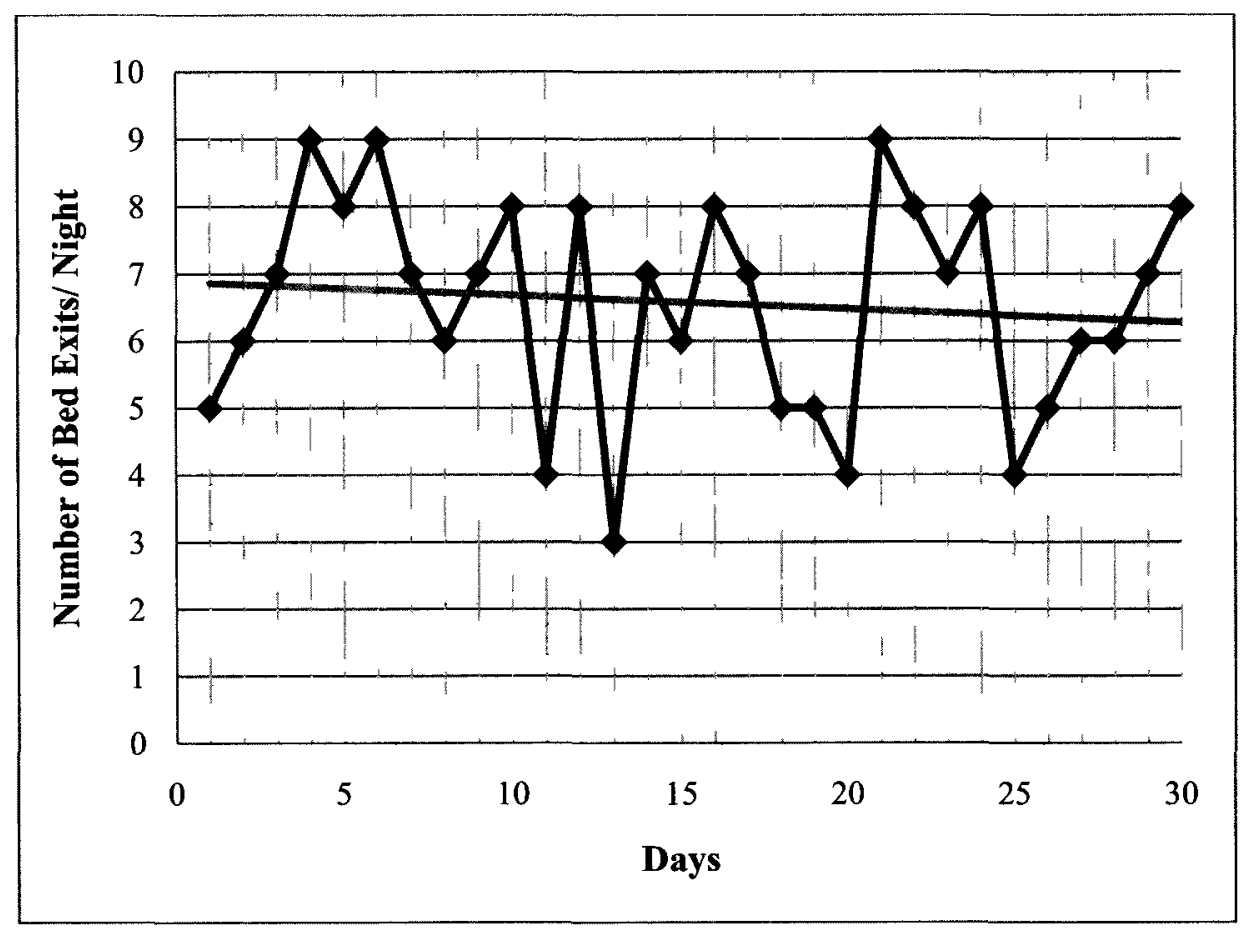

Figure 5.3: Least-square fit of a home care client's bed exit data

The least-squares fit method displays the correct trend for the data in Figure 5.3 but the technique is not always appropriate for use in populating the data for a home care decision support system. Therefore, techniques which can provide greater detail to the underlying data trends will be investigated.

\subsubsection{Data Trends}

Figure 5.3 illustrates one type of basic shape of a trend, a decreasing trend. There are six other basic shapes, referred to as primitives, which also describe trends. These other six are $\{$ Increasing, Steady, Concave Up, Concave Down, Convex Up, and Convex Down $\}$. All seven primitives can be uniquely characterized by constant value of the first and second derivatives and are shown in Figure 5.4 [86]. 

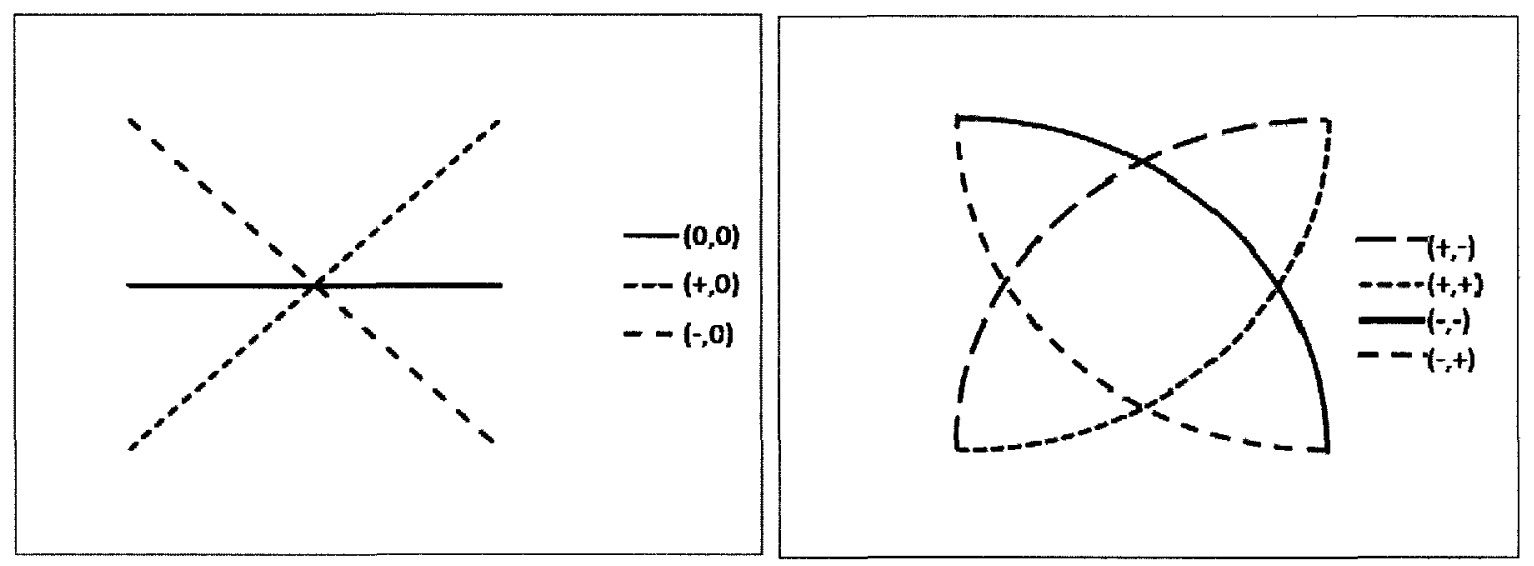

Figure 5.4: Primitives (adapted from [86])

The focus of the work presented here will be on using the primitives \{Steady, Increasing, Decreasing \}, to illustrate trends in data. The trends presented to each of the data mining techniques will include all seven primitives in order to realistically represent potential data presented to the data mining techniques. Figure 5.5 displays the five trends which will be used by each data mining technique in the simulations.

The sampling frequency of a health parameter affects the results of both data mining techniques. The sampling frequency can either be:

1. The interval of time needed to recognize a change in a particular health parameter and considering Nyquist criteria; or

2. The interval of time indicated by the home care clinicians for a particular health parameter

The samples in the trends shown in Figure 5.5 are meant to represent events. For example, the data from a bed pressure sensor mat has been pre-processed and the samples are considered counts of bed pressure mat information (e.g. number of bed exits per day) and not a continuous signal. 

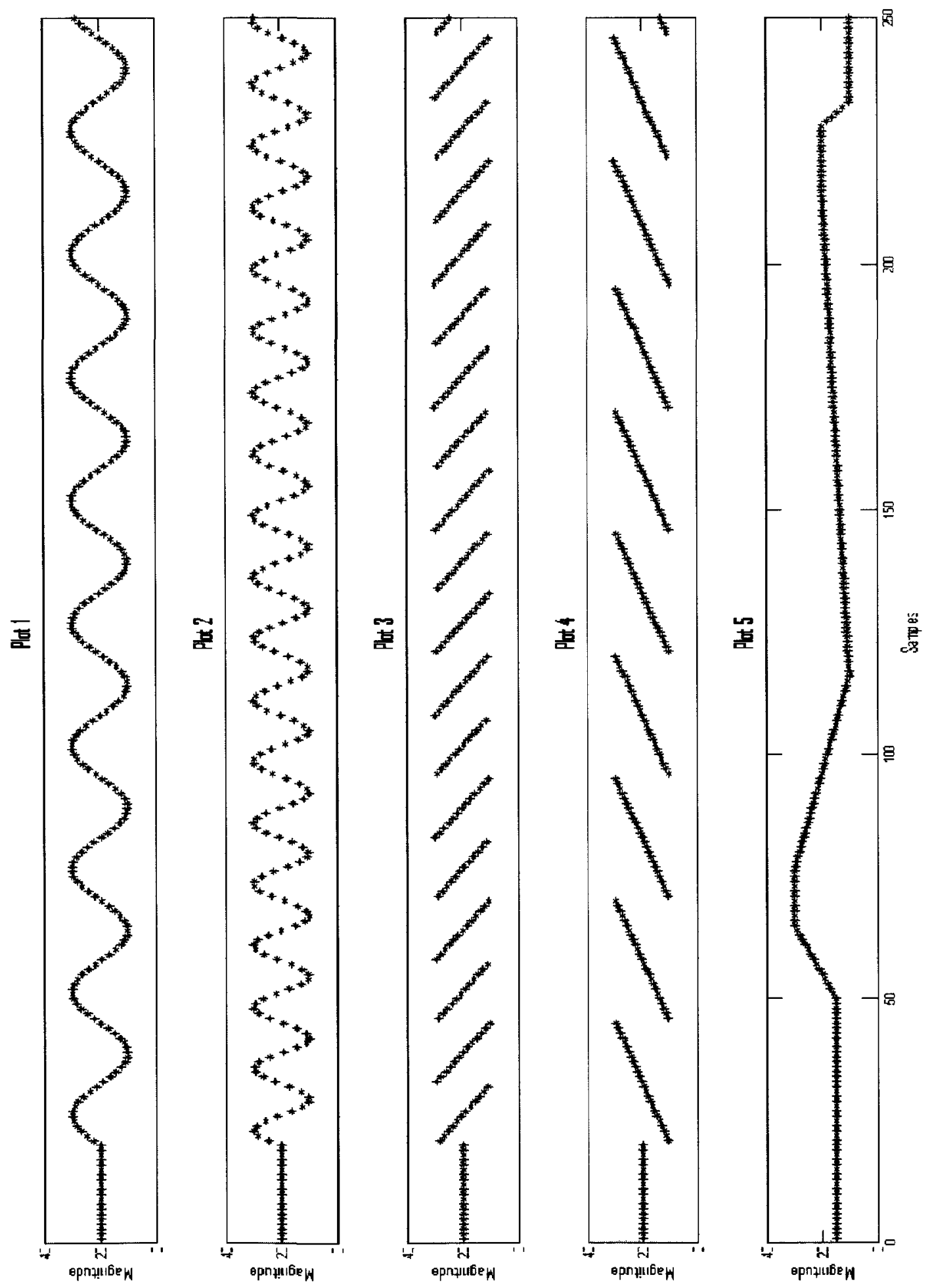

Figure 5.5: Trend types for simulation (Plots 1 - 5) 


\subsubsection{Data Trends with Noise}

Gaussian noise was chosen as the type of noise as an example to demonstrate the variation which may exist in a home care client's health data. Gaussian noise "is statistical noise that has a probability density function of the normal distribution" [130]. In order to test the data mining techniques in a more realistic fashion, various levels of noise will be added to each of the five trend types (i.e. Plots 1-5) (Figure 5.5).

Five levels of Gaussian noise were chosen to test the ability of each data mining technique to accurately discover the underlying trends of each trend type. The percentage of noise added to each trend type was determined from the maximum value reached by the trend types. An example of a trend type, Plot 5, with five different levels of noise can be seen in Figure 5.6. 


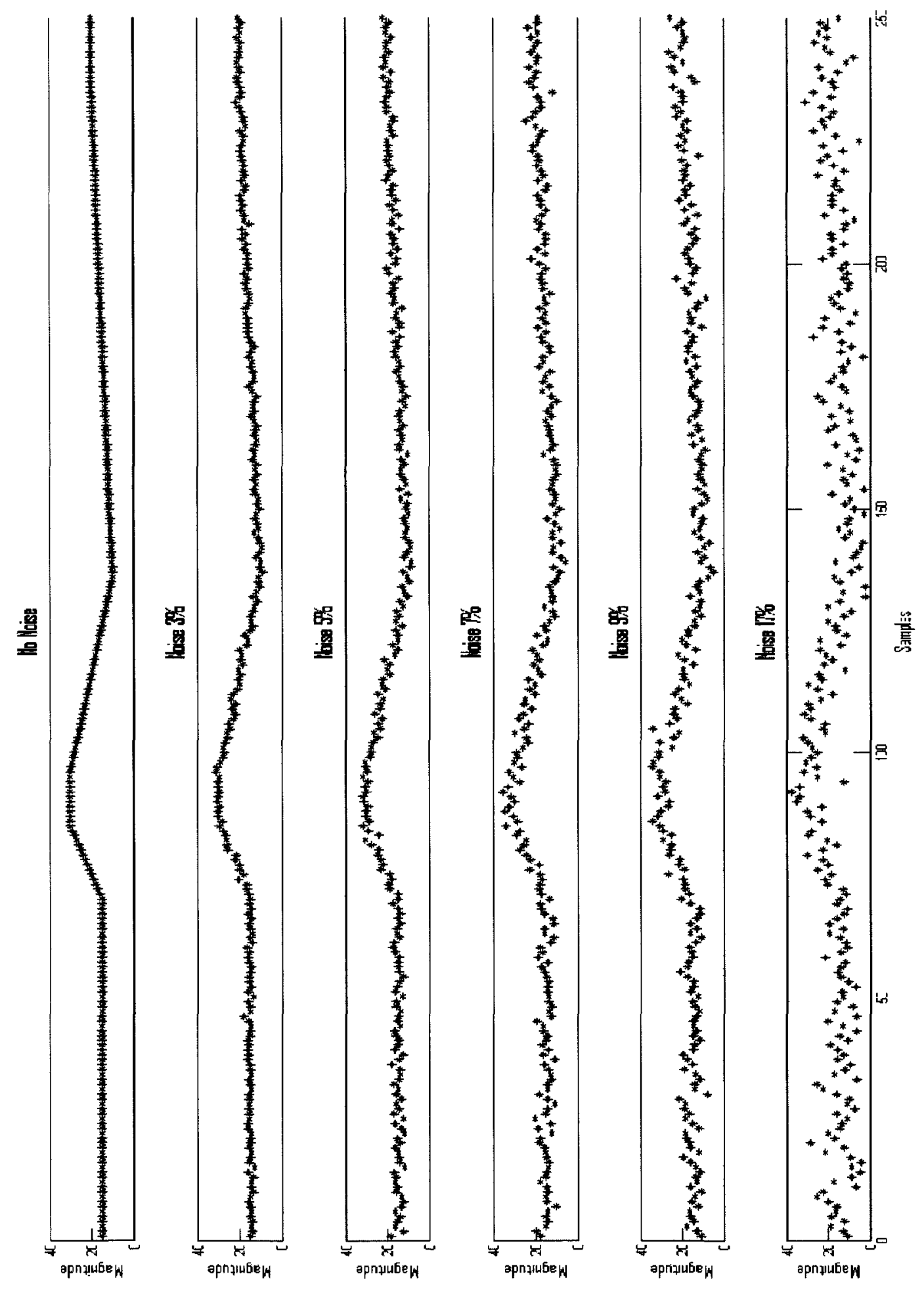

Figure 5.6: Plot 5 trend type with different levels of Gaussian noise 


\subsection{Detecting Trends Using a Segmentation Algorithm}

A simple trend line fit is not always appropriate because it requires a fixed duration moving window [131]. Choosing a time window, such as the case in linear filtering methods like a low-pass filter or a moving averager, is difficult because of rapid variations in data, for example, those such as step variations. Both these types of variations and slow trends are important to analyze in a home care client's data and therefore, a technique which does not require this selection will be presented. This technique splits the data into linear segments by a segmentation algorithm and is detailed in the following section [126].

\subsubsection{Description of the Algorithm}

As outlined by Charbonnier and Gentil [131], at each sampling time, there are four steps to extract episodes from a patient's data:

1. On-line segmentation of data into linear segments;

2. Classification of the latest segment in to seven temporal shapes: steady, increasing, decreasing, positive or negative step, increasing/ decreasing or decreasing/ increasing

3. Transformation of the shape obtained into semi-quantitative episodes, using primitives \{Steady, Increasing, Decreasing\}.

4. Aggregation of the current episode with the previous ones to form the longest possible episode

This section provides a detailed explanation of each step and the modifications suggested of the algorithm presented in the IEEE Transactions on Biomedical Engineering [126] for the application of home care remote patient monitoring. 


\section{On-line segmentation of data}

Charbonnier [132] developed a segmentation algorithm that consists of dividing data into consecutive line segments of the form:

$$
y(t)=p\left(t-t_{0}\right)+y_{0}
$$

Where $t_{0}=$ time when the segment begins

$p=$ the segment's slope

$y=$ the ordinate at time $t_{0}$

The "algorithm determines on-line the moment when the current linear approximation is no longer acceptable and when the new linear function that now best fits the data should be calculated" [131]. The cumulative sum (CUSUM) technique is used to detect the acceptability. This technique reacts quickly to sudden changes therefore, it is sensitive enough to capture patient data behaviour changes.

The first step is to assume that at time, $t_{\text {seg }}^{i-1}$, the characteristics of a new linear approximation have been calculated (Figure 5.7). These characteristics are:

$$
\begin{gathered}
p_{b}^{i-1}=\text { slope } \\
t_{0}^{i-1}=\text { beginning time } \\
y_{0}^{i-1}=\text { the ordinate at time } t_{0}^{i-1}
\end{gathered}
$$

At time, $t_{j}, t_{j}>t_{\text {seg }}^{i-1}$, the model output is given by:

$$
y\left(t_{j}\right)=p^{i-1}\left(t_{j}-t_{0}^{i-1}\right)+y_{0}^{i-1}
$$

Equation (14) calculates the difference between the signal measured at time $t_{j}$, $y_{\text {meas }}\left(t_{j}\right)$, and the model output.

$$
e\left(t_{j}\right)=y_{\text {meas }}\left(t_{j}\right)-y\left(t_{j}\right)
$$


Equation (15) is used to calculate the cumulative sum of the differences from time $t_{\text {seg }}^{i-1}$.

$$
\operatorname{CUSUM}\left(t_{j}\right)=\operatorname{CUSUM}\left(t_{j}-1\right)+e\left(t_{j}\right)=\sum_{t_{k}=t_{s e g}^{i-1}}^{t_{j}} e\left(t_{k}\right)
$$

Two thresholds must be determined before employing the segmentation algorithm. These thresholds are $t h_{1}$ and $t h_{2}$ and they need to be chosen using a priori knowledge on the variable behaviour in normal operation [131].

$T h_{1}$ tunes the decomposition into linear segments and takes the process noise into account [126]. In the example used at the beginning of this chapter, the data in this research has already been pre-processed and is considered counts of bed pressure mat information. Therefore, "noise" can be considered as half the client's 'normal' or stable range or something related to the range in which most of the data lies in. For example, in section 5.2.1 a method of detecting outliers is to look for data points more than a certain number of standard deviations, $\sigma$, from the mean, $\mu$. Thus, it can be also be used for calculating $t h_{I}$.

$T h_{2}$ also tunes the decomposition into linear segments and determines the filtering effect [126]. This threshold is adjusted as a "function of the delay required to detect a variable change" [131]. This threshold corresponds to the "integral of the change to be detected on a time interval equal to the delay necessary to detect this change and can be modified depending on the kind of transients that should be detected (duration $D$ and amplitude $A$ )" [131].

In order to determine if a model is acceptable or not, at each sampling time $t_{j}$ the absolute value of the CUSUM is compared to these two thresholds using the following conditions [131]: 
- If $\left|C U S U M\left(t_{j}\right)\right| \leq t h_{1}$, then the current model is acceptable;

- If $t h_{1}<\left|C U S U M\left(t_{j}\right)\right| \leq t h_{2}$, then $y_{\text {meas }}\left(t_{j}\right)$, and $t_{j}$ are memorized into a matrix $M$;

- If $\left|\operatorname{CUSUM}\left(t_{j}\right)\right| \leq t h_{2}$, then the linear model is no longer acceptable. The algorithm then uses the values contained in $M$ to calculate the characteristics of a new linear least-squares approximation (i.e. $p^{i}, t_{0}^{i}, y_{o}^{i}$ ). One stipulation of this condition is that there must be at least three values stored in $M$. The time, $t_{j}$, corresponds to new segmentation time, $t_{\text {seg }}^{i-1}$. The new segmentation time The CUSUM is reset to zero once a new linear function has been calculated.

Figure 5.7 and Figure 5.8 illustrate the parameters of the segmentation algorithm. The signal and model graph (Figure 5.7) displays the signal and its linear model and model extrapolation. This graph also shows the new model calculated in correspondence to the CUSUM crossing $t h_{2}$ at sample 37 in Figure 5.8.

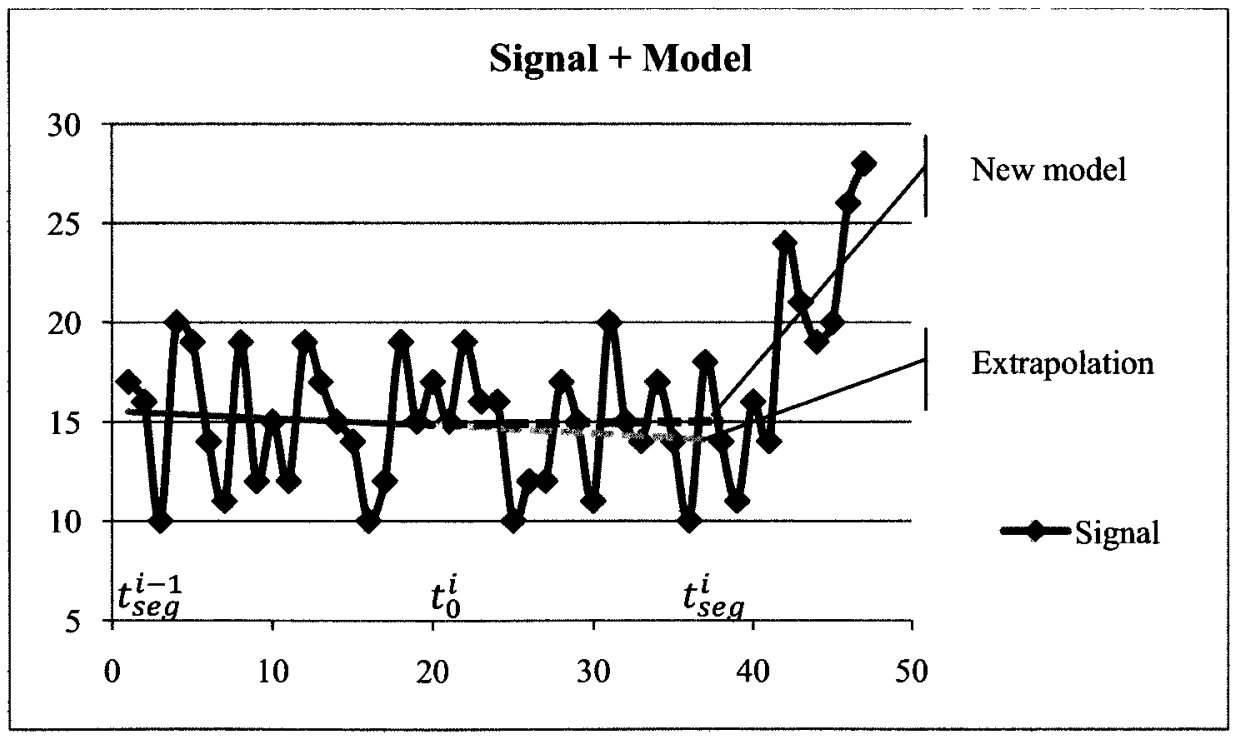

Figure 5.7: Example of a signal, extrapolation, and new model of the segmentation technique 


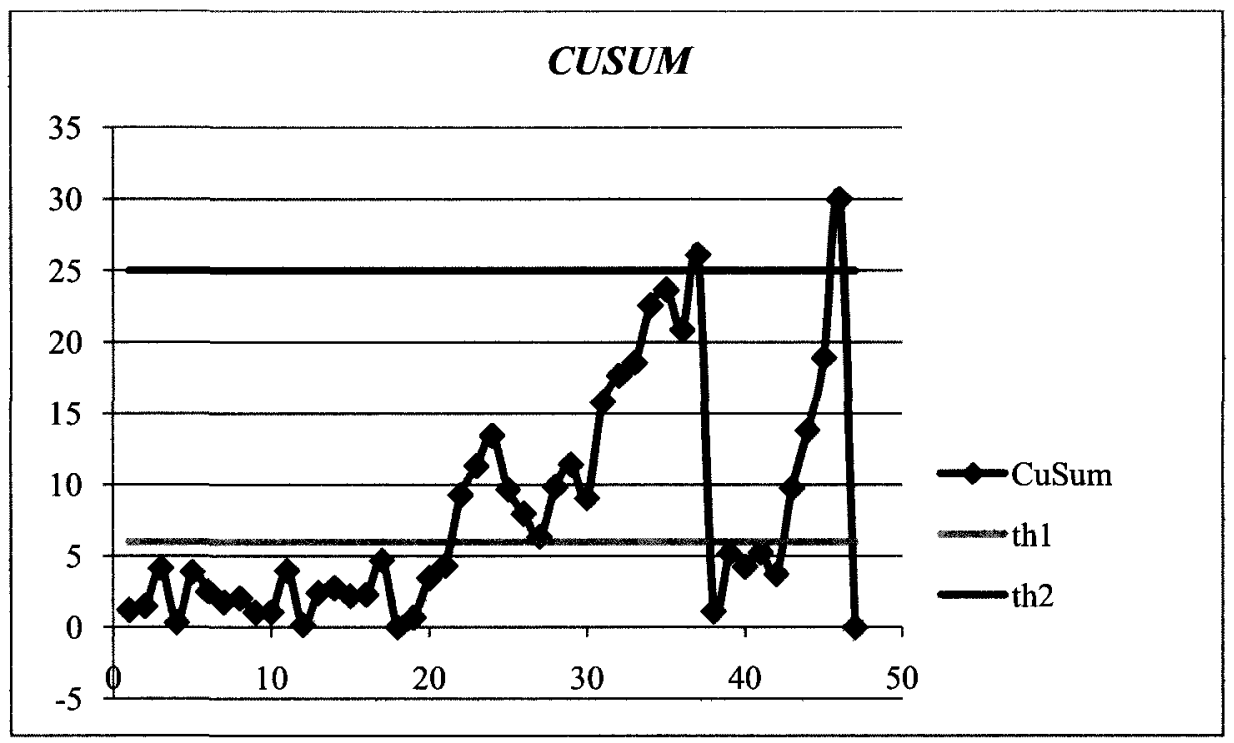

Figure 5.8: Example of the CUSUM of the segmentation technique

\section{Classification of the last two segments into a temporal shape}

After the segmentation algorithm has calculated a new segment, the segment forms a shape with the previous segment into one of the seven temporal shapes described above: steady, increasing, decreasing, positive or negative step, increasing/ decreasing or decreasing/ increasing.

According to Charbonnier and Gentil [131], a shape can be defined by three features:

1. $I$, the total increase (or decrease)

2. $I_{d}$, the increase (or decrease) due to the discontinuity: $I_{d}=y_{0}^{i}-y_{e}^{i-1}$,

3. $I_{s}$, the increase (or decrease) due to the slope: $I_{s}=y_{e}^{i}-y_{0}^{i}$

Several features need to be extracted from the current segment and the previous one for classification. These features are displayed in Figure 5.9. 


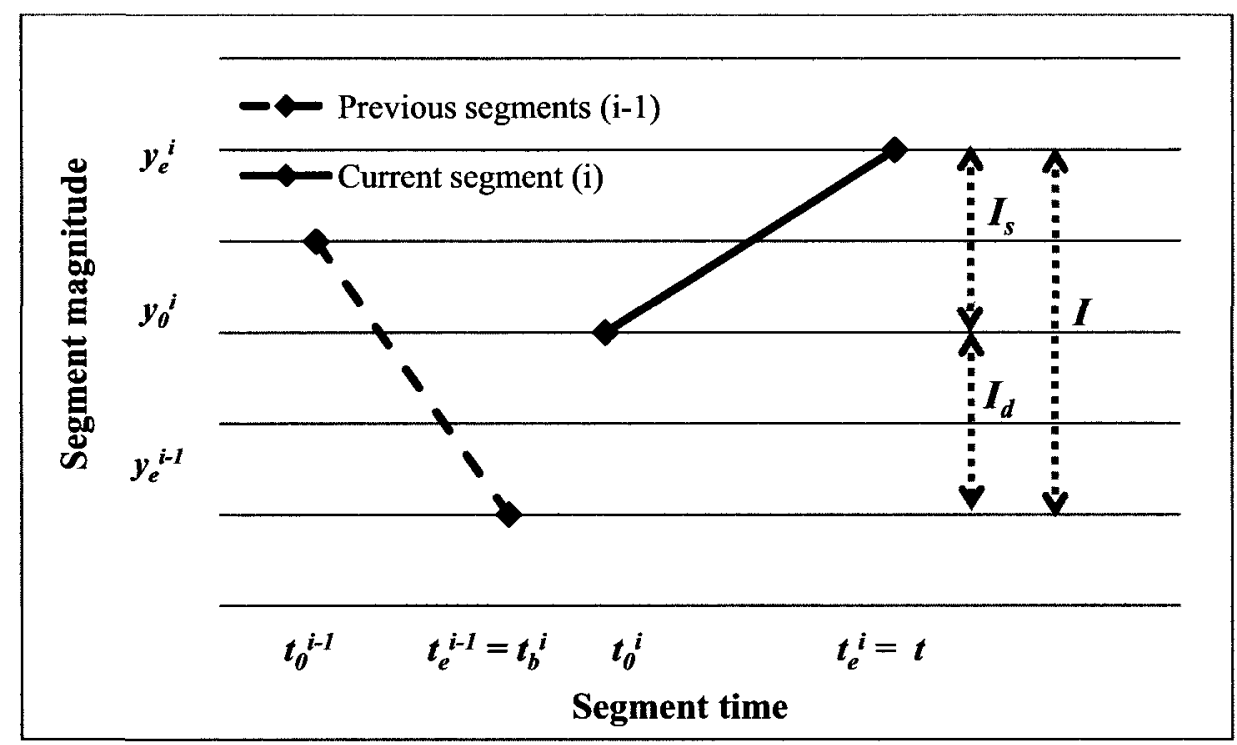

Figure 5.9: Classification features extracted from two consecutive segments (adapted from [131])

In order to classify the segments using the three features, a set of rules are utilized (Table 5.1). These rules require that two other thresholds be determined, $t h_{h c}$ and $t h_{h s}$. The threshold $t h_{h c}$ depends on what clinicians consider a significant variation [131]. The threshold $t h_{h s}$ determines if a shape is \{Increasing, Decreasing or Steady\} [133].

Table 5.1: Classification rules (adapted from [133], [65])

\begin{tabular}{|c|c|c|c|c|c|}
\hline \multicolumn{6}{|c|}{ Process Steps } \\
\hline 1 & & 2 & 3 & \multicolumn{2}{|r|}{4} \\
\hline \multirow{3}{*}{$\left|I_{d}\right|<t h_{h c}$} & \multirow{3}{*}{ 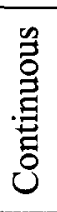 } & \multirow{2}{*}{$|I|>t h_{h s}$} & $I>0$ & \multicolumn{2}{|r|}{ Increasing } \\
\hline & & & $I<0$ & \multicolumn{2}{|r|}{ Decreasing } \\
\hline & & $|I| \leq t h_{h s}$ & \multicolumn{3}{|c|}{ Steady } \\
\hline \multirow{6}{*}{$\left|I_{d}\right| \geq t h_{h c}$} & \multirow{6}{*}{ 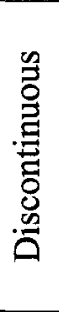 } & \multirow{2}{*}{$\left|I_{s}\right|<t h_{h s}$} & \multirow{2}{*}{ 离 } & $I_{d}>0$ & Positive Step \\
\hline & & & & $I_{d}<0$ & Negative Step \\
\hline & & \multirow{4}{*}{$\left|I_{s}\right| \geq t h_{h s}$} & \multirow{2}{*}{$\operatorname{Sign}\left(I_{d \cdot} I_{s}\right)>0$} & $I_{d}>0$ & Increasing \\
\hline & & & & $I_{d}<0$ & Decreasing \\
\hline & & & \multirow{2}{*}{$\operatorname{Sign}\left(I_{d \cdot} I_{s}\right)<0$} & $I_{d}>0$ & Increasing/ decreasing transient \\
\hline & & & & $I_{d}<0$ & Decreasing/ increasing transient \\
\hline
\end{tabular}




\section{Transformation of shapes into semi-quantitative episodes}

This step uses the temporal shapes introduced in the previous step and transforms them into semi-quantitative episodes. This conversion helps to create a more easily understandable trend of a patient's data. If a shape has been classified as SSteady, Increasing, or Decreasing\}, it is converted into one episode identified by the same term [131]. Therefore, an episode is defined by the set:

$$
\begin{gathered}
\left\{\text { Primitive, } t_{b}^{i}, y_{b}^{i}, t_{e}^{i}, y_{e}^{i}\right\} \\
\text { Where } t_{b}^{i}=\text { start time } \\
y_{e}^{i-1}=\text { start value at the beginning } \\
t_{e}^{i}=\text { end time } \\
y_{e}^{i}=\text { end value }
\end{gathered}
$$

Once an episode ends, its end time becomes the time at which the next episode starts (i.e. $t_{b}^{i+1}=t_{e}^{i}$ ). The current time, $t_{c}$, is the end of the latest episode.

If a Transient or Step shape was identified in the previous step, it is split into two episodes. A Transient is considered a short-lived oscillation and a Step a quick increase or decrease followed by a Steady portion. Each of the episodes is then correlated with one of the three linguistic terms [131]. For example, a Negative Step (respectively, Positive) becomes the following two episodes:

$$
\begin{gathered}
\left\{\text { Decreasing (respectively, Increasing), } t_{e}(i-1), y_{e}(i-1), y_{0}(i)\right\} \\
\left\{\text { Steady, } t_{o}(i), y_{0}(i), y_{e}(i)\right\}
\end{gathered}
$$

By initially determining a precise shape and then a more simplified one, the process allows the signal discontinuities to be described more accurately. 


\section{Aggregation of episodes}

The purpose of this step is to connect the most recent episode with the previous one in order to form the longest possible episode. An example of aggregation rules is shown in Table 5.2 and all the possible aggregations are shown in Table 5.3.

Table 5.2: An example of aggregation rules [131]

IF the previous episode is $\left\{\right.$ Increasing, $\left.t_{b}^{i-1}, y_{b}^{i-1}, t_{e}^{i-1}, y_{e}^{i-1}\right\}$

AND the current episode is $\left\{\right.$ Increasing, $\left.t_{b}^{i}, y_{b}^{i}, t_{e}^{i}, y_{e}^{i}\right\}$

THEN the new episode is $\left\{\right.$ Increasing, $\left.t_{b}^{i-1}, y_{b}^{i-1}, t_{e}^{i}, y_{e}^{i}\right\}$

ELSE IF the current episode is Steady or Decreasing

THEN it cannot be aggregated and a new episode is generated: \{lncreasing, $\left.t_{b}^{i-1}, y_{b}^{i-1}, t_{e}^{i-1}, y_{e}^{i-1}\right\} ;\left\{\right.$ Steady or Decreasing, $\left.t_{b}^{i}, y_{b}^{i}, t_{e}^{i}, y_{e}^{i}\right\}$

Table 5.3: Possible aggregations [131], [133]

Increasing + Increasing = Increasing
Decreasing + Decreasing = Decreasing
Steady + Steady = Steady if $\left|y_{e}^{i}-y_{b}^{i-1}\right|<t h_{h c}$
Steady + Steady = Increasing if $\left|y_{e}^{i}-y_{b}^{i-1}\right| \geq t h_{h c}$
Steady + Steady = Decreasing if $\left|y_{e}^{i}-y_{b}^{i-1}\right| \geq t h_{h c}$

The last two possible aggregations in Table 5.3 allow for the detection of slow drifts in a patient's data. If a Steady episode is detected, it is classified into either a flat or bent episode. A bent episode is detected if the value of the difference between the end and beginning values of the episode is superior to $25 \%$ of the value corresponding to a significant change (i.e. $0.25 \times t h_{h c}$ ). Consecutive bent episodes are memorized into a matrix N1. If the difference between the end value of the last episode in $N 1$ and the beginning value of the first episode in $N 1$ is greater than $t h_{h c}$, it is an increasing episode and a slow drift has been detected. $N 1$ is emptied if a flat, Increasing, or Decreasing episode is detected and then presented for aggregation [133]. 
Equally important to detecting an increasing slow drift, is detecting a decreasing slow drift. For example, detecting this type of drift in the case of number of bed exits would mean the home care client's condition could be improving. Therefore, an additional matrix $N 2$ is needed in the algorithm in order to store bent episodes of a decreasing nature. A bent decreasing episode is detected if the value of the difference between the end and beginning values of the episode is less than $-25 \%$ of the value corresponding to a significant change (i.e. $-0.25 \times t h_{h c}$ ). Bent episodes are memorized in the same fashion as $N 1$ and again a verification of the absolute difference between the end value of the last episode in $N 2$ and the beginning value of the first episode in $N 2$ occurs in order to determine if it is greater than $t h_{h c} . N 2$ is also emptied in a similar fashion to $N 1$.

Another slow drift detection method is also in utilized and is placed after the aggregation step. If a Steady episode has just been aggregated, the difference between the values at the end and beginning of the episode is calculated. If it is greater than $150 \%$ of $t h_{h c}$, an error has been detected and the episode needs to be transformed into an Increasing one [133].

Similarly, the treatment of a decreasing slow drift is also checked after the aggregation step. In the case that a Steady episode has just been aggregate, the same calculation occurs as above and if the difference is less than $-150 \%$ of $t h_{h c}$, an error has been detected and the episode is renamed as a Decreasing one.

In order to detect a slow drift, at least two Steady episodes must be aggregated and therefore, it takes more time. This limitation is not critical since the event of a slow drift does not correspond to urgent care needed for a patient [133]. 
Once two successive Steady episodes are aggregate into a new one, the median value of the signal during the entire episode must be calculated. This value replaces the ends of the episode and in order to preserve continuity, it replaces the end value of the previous episode. This allows for a more understandable display because a Steady episode is then represented by a flat line and can be identified by a clinician in a glance [131], [133].

\subsubsection{Simulation Results - Noisy Data}

A MATLAB script was written and run with MATLAB version 7.8.0 (R2009a) to mine simulated pressure signals from a bed pressure sensor mat. The signals were created in MATLAB as well and consisted of a variety of increasing, decreasing, step and steady segments (Figure 5.5). The length of data was arbitrarily chosen to consist of 250 samples.

The data collected from a Tactex Bed Occupancy Sensor by the Tactex CCL Data Acquisition software is logged as an ASCII comma delimited file. Therefore, the simulated signals were saved in this format and then imported into MATLAB for each simulation.

The tuning parameters of the segmentation algorithm must be adapted to each individual home care client. The assumption here is that the 'noise' represents the client's 'normal' variance. This noise varies for each individual client and the changes which need to be detected are also unique.

The process for selecting the appropriate values of the tuning parameters is summarized below. 


\section{Threshold: $t h_{1}$}

The testing values of the parameter $t h_{l}$ were chosen by taking into account the range of 'normal' variance generally corrupting the variable considered. This variance relates to the noise introduced in 5.2.3. Care was taken not to choose a value which was too small because then the algorithm would store data in matrix $M$ (see Step 1 in 5.3 .1 ) when no real change was observed which slightly truncates the signal during transitions. If too large of a value was selected, the algorithm would not store the data when a change already occurred. This would cause a late detection at the beginning for variations, most notably in step variations. Additionally, the difference between $t h_{1}$ and $t h_{2}$ needed to be large enough so that sufficient values could be stored in $M$ to calculate a new segment [126].

\section{Threshold: $t h_{2}$}

It has been demonstrated in Charbonnier et al. [133], that a good starting point in choosing the value for this parameter is around ten times the value of $t h_{l}$. Even so, it should be large enough so that it does not take into account noise as a change in the data and small enough not to miss any significant transients, but not so small that a new segment is calculated very often [126].

\section{Threshold: $t h_{h c}$}

This parameter needed to be set so that it could help detect any change whose amplitude was greater than itself and considered significant. If $t h_{h c}$ was set too small

compared to the noise level corrupting the signal, irrelevant episodes (succession of Increasing-Decreasing) would be mined. If this value was too large, "significant 
variations may be missed and the corresponding episode may be Steady" [131]. This threshold helps to separate continuous and discontinuous shapes from one another [133].

\section{Threshold: $t h_{h s}$}

This parameter helps to separate steady shapes from increasing or decreasing shapes and has demonstrated to work well when equal to $t h_{h c}$ [133]. Therefore, similar testing values for the two thresholds were chosen.

Thus, using the original data sets from Figure 5.5 for the model to match, the optimal values for the tuning parameters can be selected by testing $x^{n}$ combinations of values where, $x$ equals the number of values tested (3) and $n$ equals the number of thresholds (4) (Table 5.4). The combination of values with the overall smallest combined root-mean-square error (RMSE) was selected for each plot.

RMSE is often used to measure the accuracy of estimates by measuring the average magnitude of the error. The error is the difference between the estimate, known as the "model" in this research, and the observed or true value, this being the un-noisy signal. The differences are each squared and then averaged over the sample. Lastly, the square root of the average is taken. Caution is advised when using the RMSE for accuracy comparison, as is the case here. The RMSE can be very large in the case of only a few abnormal points (i.e. is gives a relatively high weight to large errors). This makes the RMSE useful when large errors are particularly undesirable. One can also use other accuracy measures such as the mean and variance of the errors as well [134]. 
Table 5.4: Testing and final values of the tuning parameters for the simulated data sets

\begin{tabular}{|c|l|c|}
\hline Tuning Parameter & \multicolumn{1}{|c|}{ Description } & Testing Values \\
\hline$t h_{1}$ & $\begin{array}{l}\text { Tunes the decomposition into linear segments and } \\
\text { takes the process noise into account [126] }\end{array}$ & $2,3,4$ \\
\hline$t h_{2}$ & $\begin{array}{l}\text { Tunes the decomposition into linear segments and } \\
\text { determines the filtering effect [126] }\end{array}$ & $25,30,35$ \\
\hline$t h h_{h c}$ & $\begin{array}{l}\text { Tunes the extraction algorithm, "any change whose } \\
\text { amplitude is greater than its value is considered } \\
\text { significant from an operator's point of view" [131] }\end{array}$ & $5,7,9$ \\
\hline$t h_{h s}$ & $\begin{array}{l}\text { Determines whether the shape is Increasing, } \\
\text { Decreasing, or Steady [58] }\end{array}$ & $5,7,9$ \\
\hline
\end{tabular}

*Tuning parameters values are not suitable for all cases and must be determined based on a priori knowledge of the parameter being monitored and if possible, tests verified with the algorithm for accuracy.

An example of the result obtained with the optimal combination of values for the tuning parameters on one of the data sets is shown in Figure 5.10 and Table 5.5. Table 5.5 represents the possible semi-qualitative results of the trend detection which can be outputted to the home care clinicians. The 'Start' and 'End' columns refer to the time a trend began (e.g. days, hours, seconds, etc.) and depend on the parameter being monitored. The 'Magnitude of the Signal' columns would also be in the appropriate units of the parameter being monitored (e.g. number of bed exit per night).

Results for the other plots can be found in Appendix B. These results can also be described by IEEE Std 181 - 2003 "Standard on Transitions, Pulses and Related Waveforms". This standard provides clear terms for transitions, pulses and related signals to aid communication and defines procedures for estimating parameters [135]. 

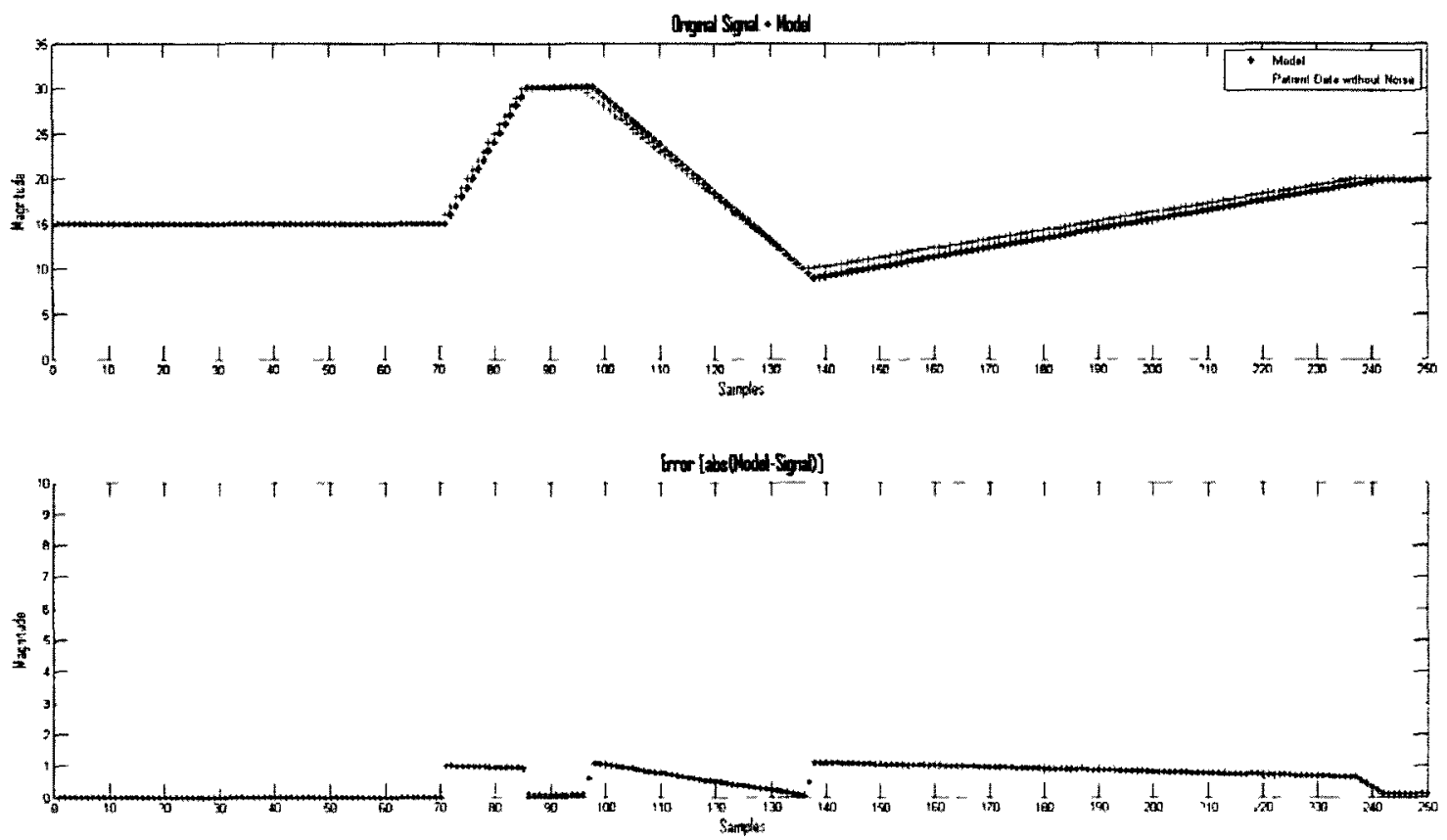

Figure 5.10: Simulated data, Plot 5, and the corresponding outputs from the segmentation algorithm

Table 5.5: Simulated data trend descriptions

\begin{tabular}{|l|c|c|c|c|c|}
\hline \multicolumn{1}{|c|}{ Trend } & Start & $\begin{array}{c}\text { Magnitude } \\
\text { of Signal }\end{array}$ & End & $\begin{array}{c}\text { Magnitude } \\
\text { of Signal }\end{array}$ & $\begin{array}{c}\text { Length of } \\
\text { Signal }\end{array}$ \\
\hline Steady & 1 & 15.0 & 70 & 15.0 & 69 \\
\hline Increasing & 70 & 15.0 & 85 & 30.0 & 15 \\
\hline Steady & 85 & 30.0 & 98 & 30.0 & 13 \\
\hline Decreasing & 98 & 30 & 136 & 10.0 & 38 \\
\hline Increasing & 136 & 10.0 & 236 & 20.0 & 100 \\
\hline Steady & 236 & 20.0 & 250 & 20.0 & 14 \\
\hline
\end{tabular}

Once the optimal combination of values for the tuning parameters was discovered, the segmentation algorithm could be presented with the varying noise levels on each trend type. Since the Gaussian noise added to each trend type was generated by a random number generator, it was important to perform this step a number of iterations in order to obtain an accurate representation of the algorithm's performance. Running the algorithm one hundred times with each noise level was completed in order to demonstrate this 
concept. An example of each noise level with Plot 5 is shown from Figure 5.11 to Figure 5.15. The following figures display the model and original signal as the top plot and the model and the noisy signal as the bottom plot. The results for the other trend types (Plots $1-4)$ can be found in Appendix C.
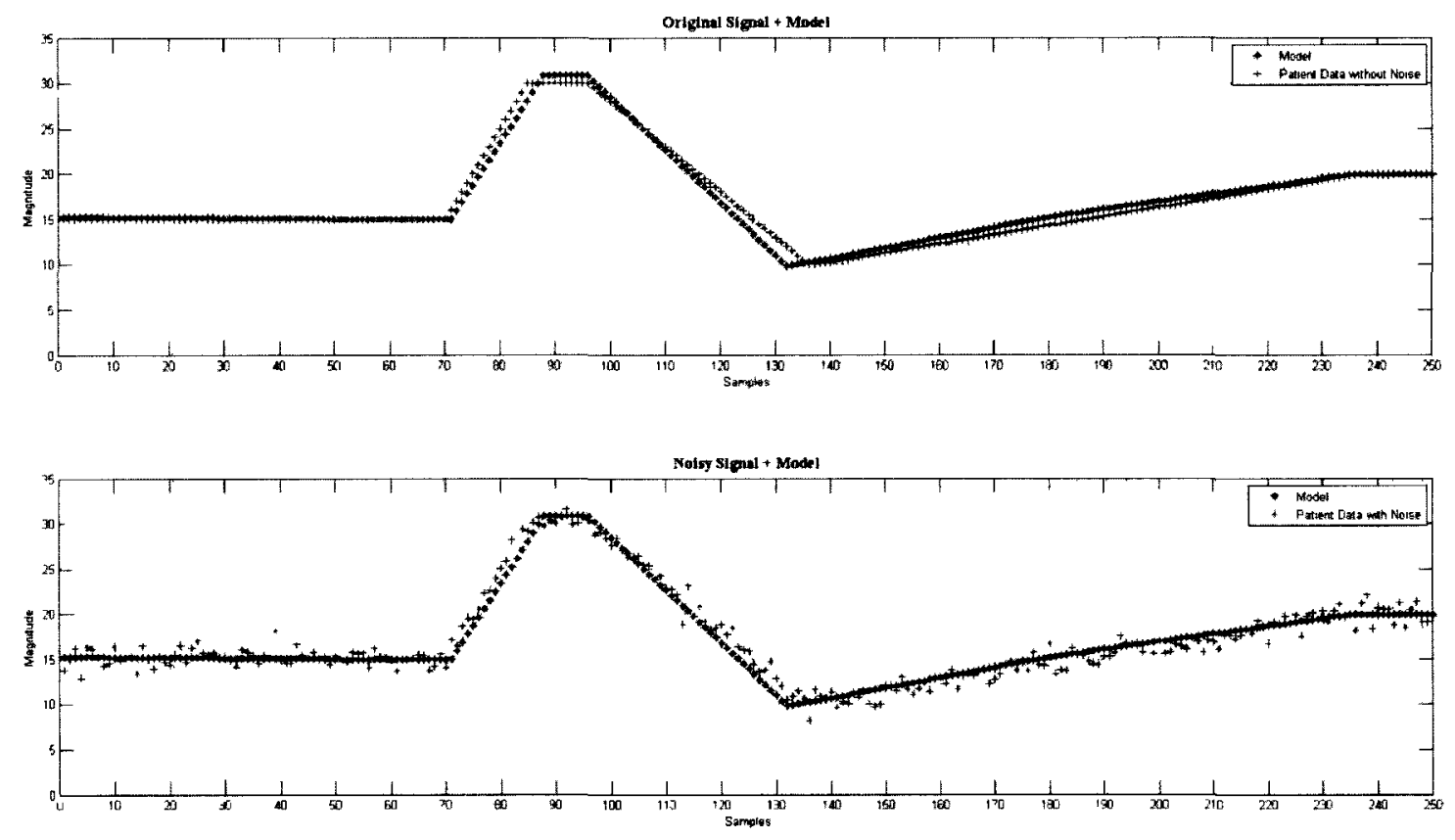

Figure 5.11: Plot 5-3\% noise 

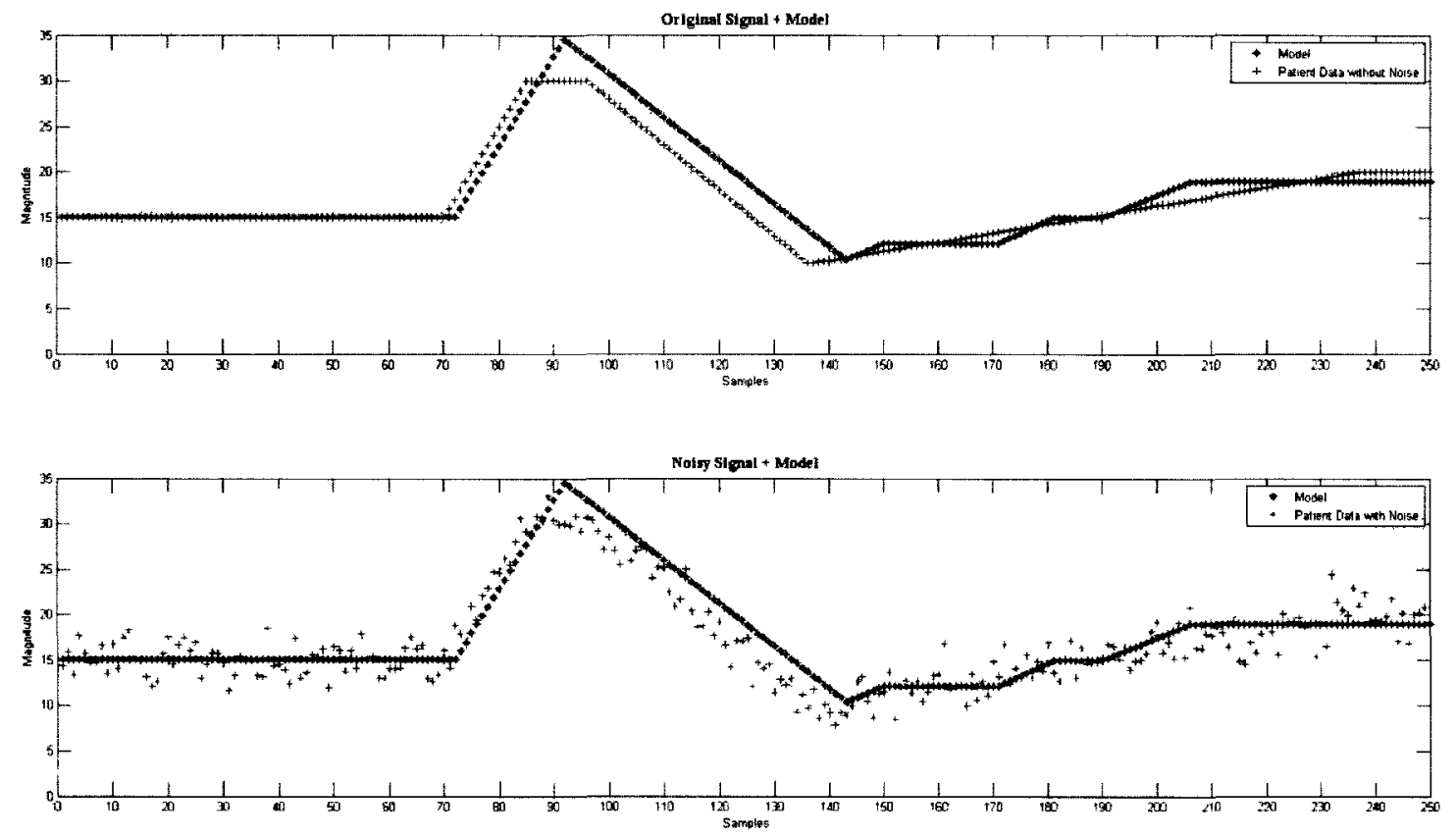

Figure 5.12: Plot 5 - 5\% noise
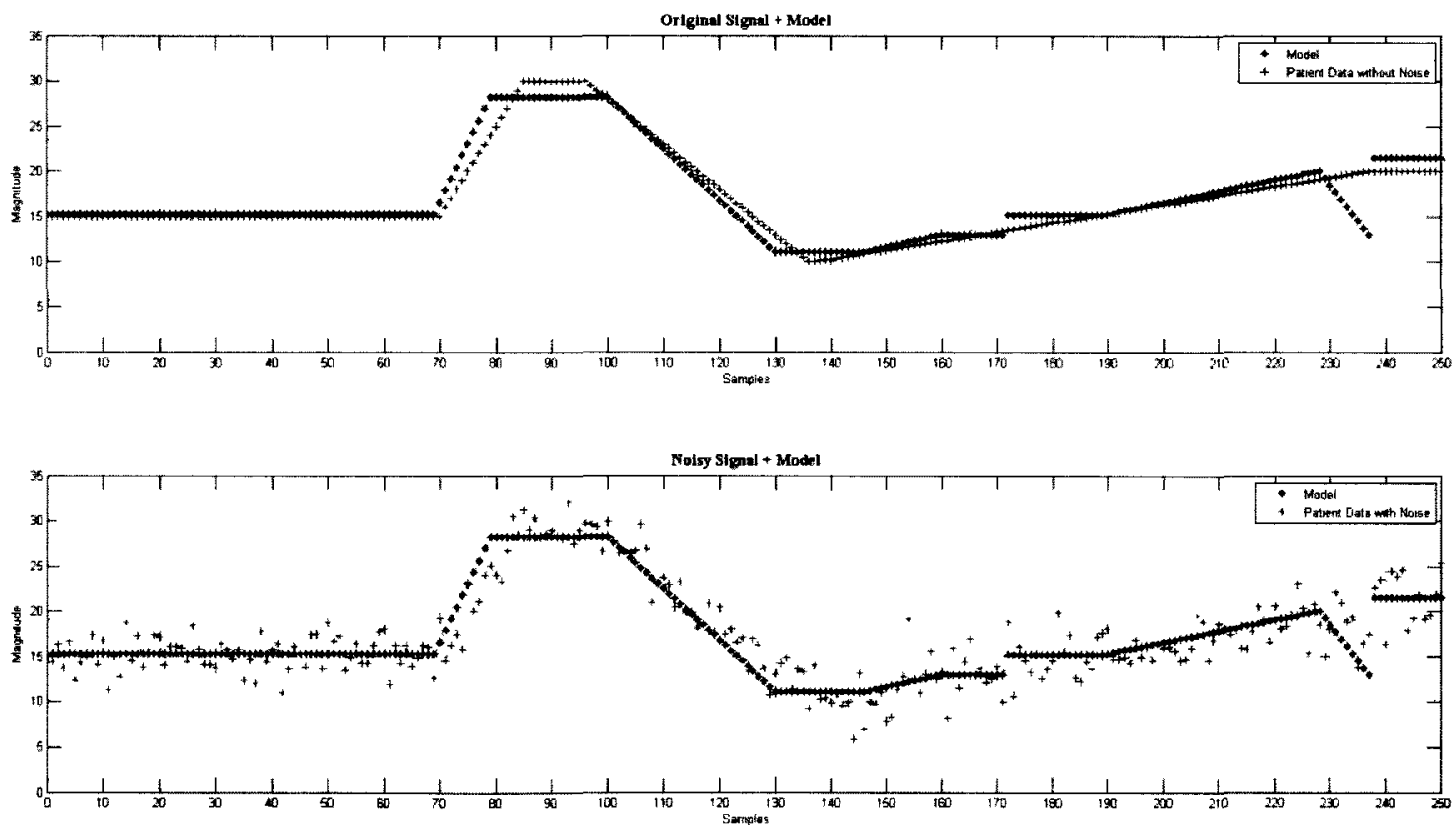

Figure 5.13: Plot 5 - 7\% noise 

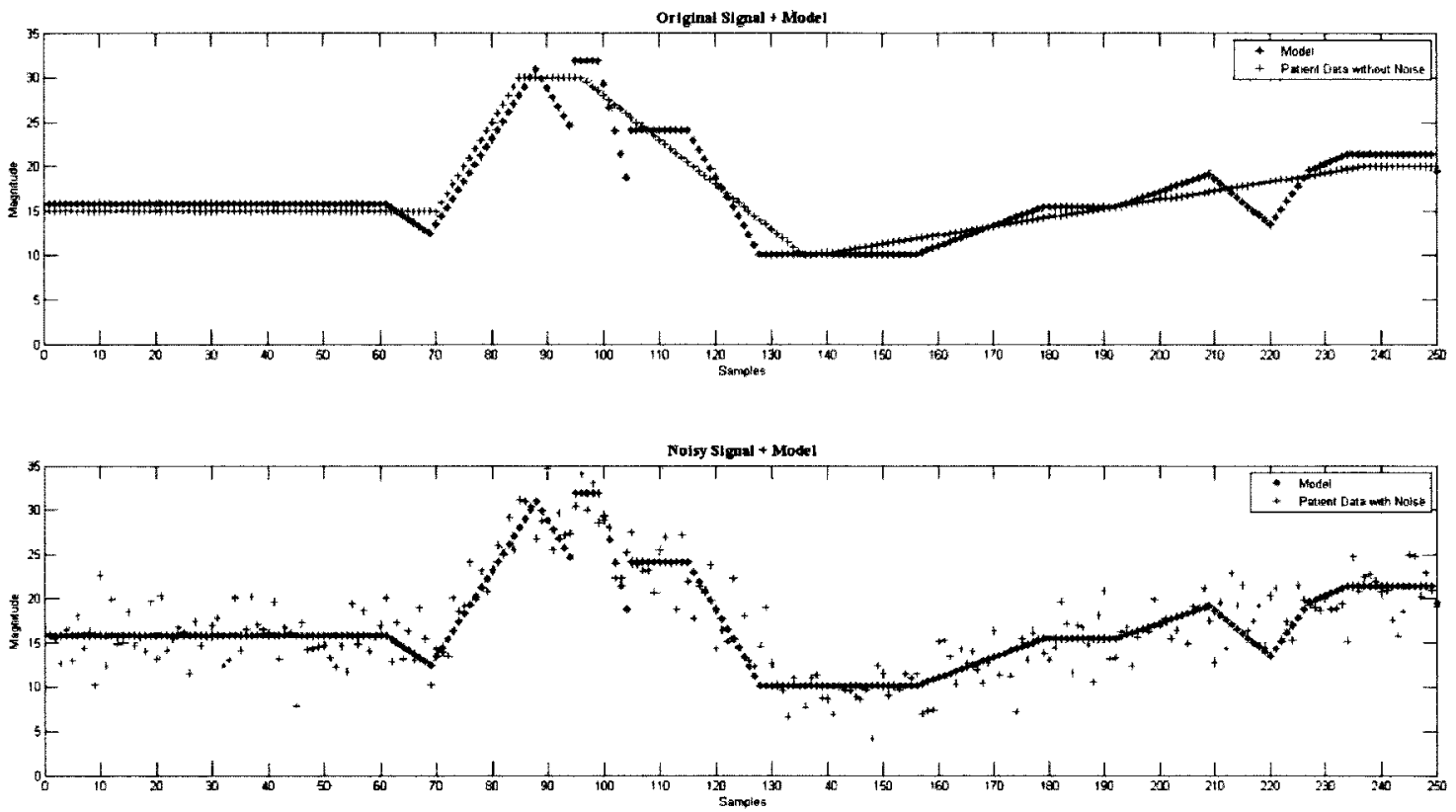

Figure 5.14: Plot 5 - 9\% noise
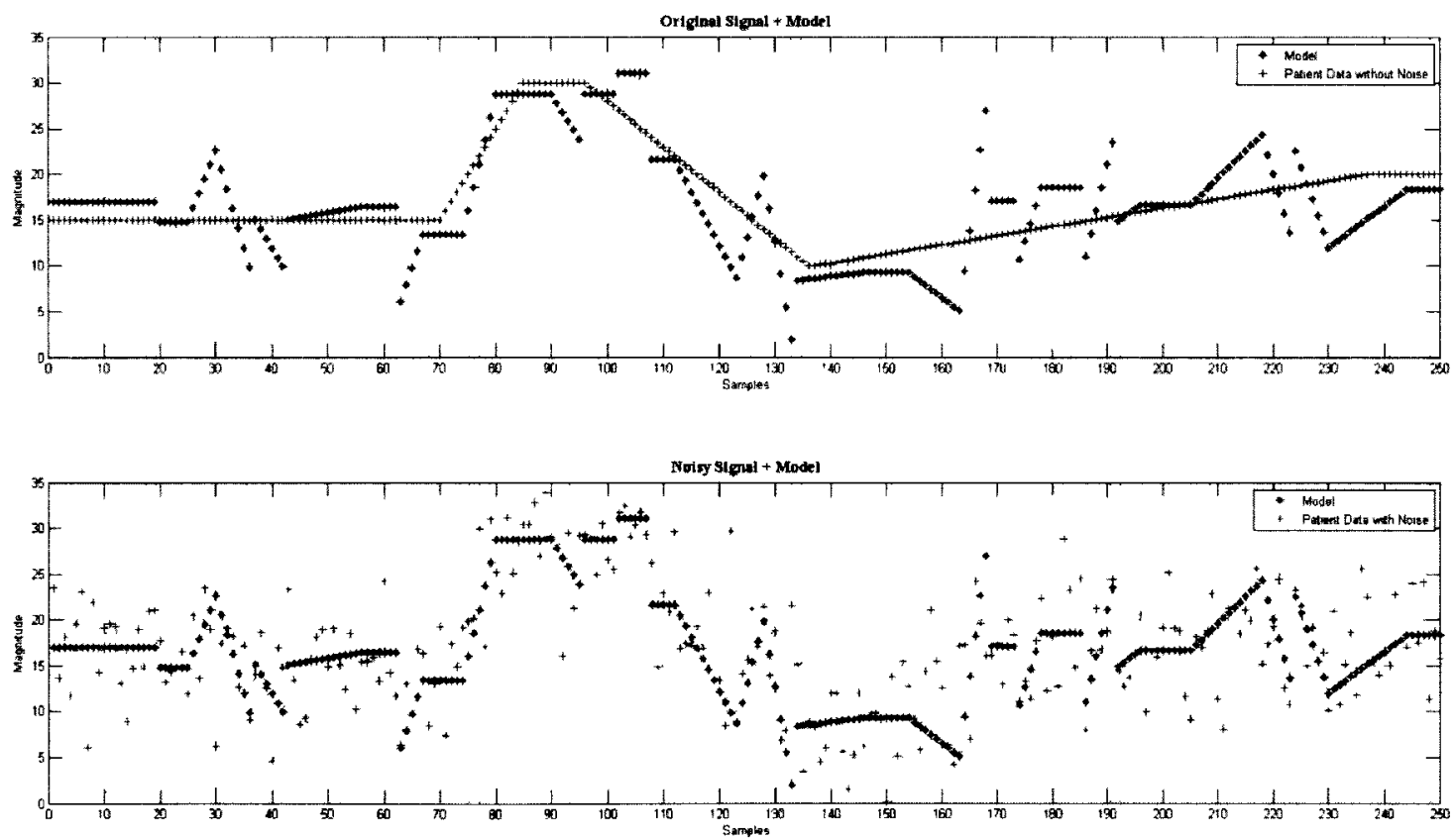

Figure 5.15: Plot 5 - 17\% noise

The performance of the data mining technique at each noise level and for each trend type can be determined by the value of root mean squared error. This value was 
averaged over the 100 passes for each trend type and the results are shown from Figure 5.16 to Figure 5.19 .

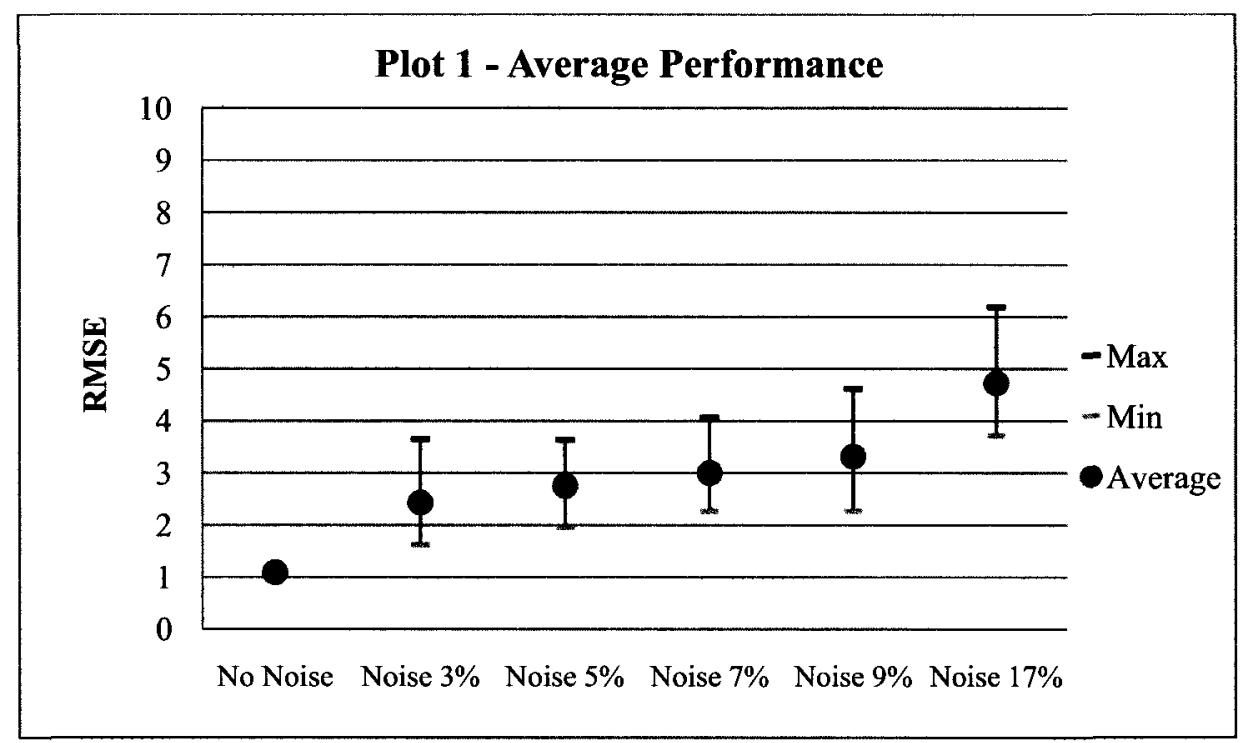

Figure 5.16: Plot 1 - Average performance at each noise level

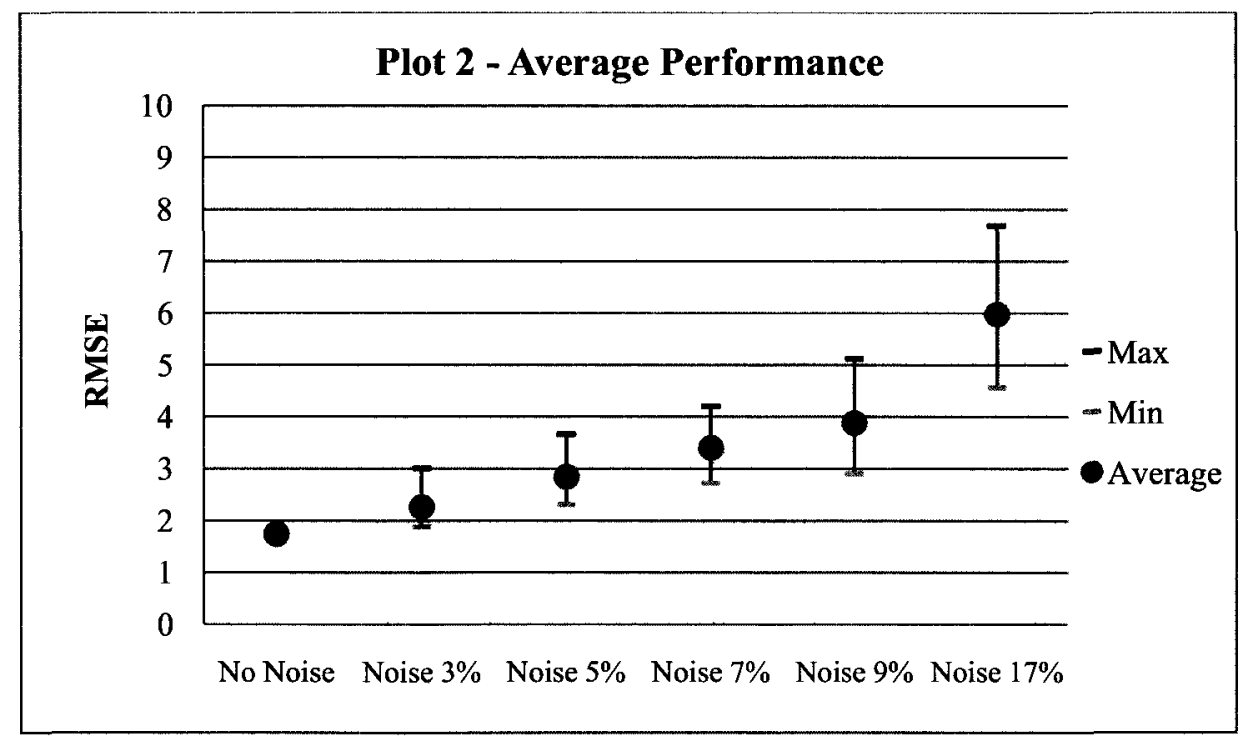

Figure 5.17: Plot 2 - Average performance at each noise level 


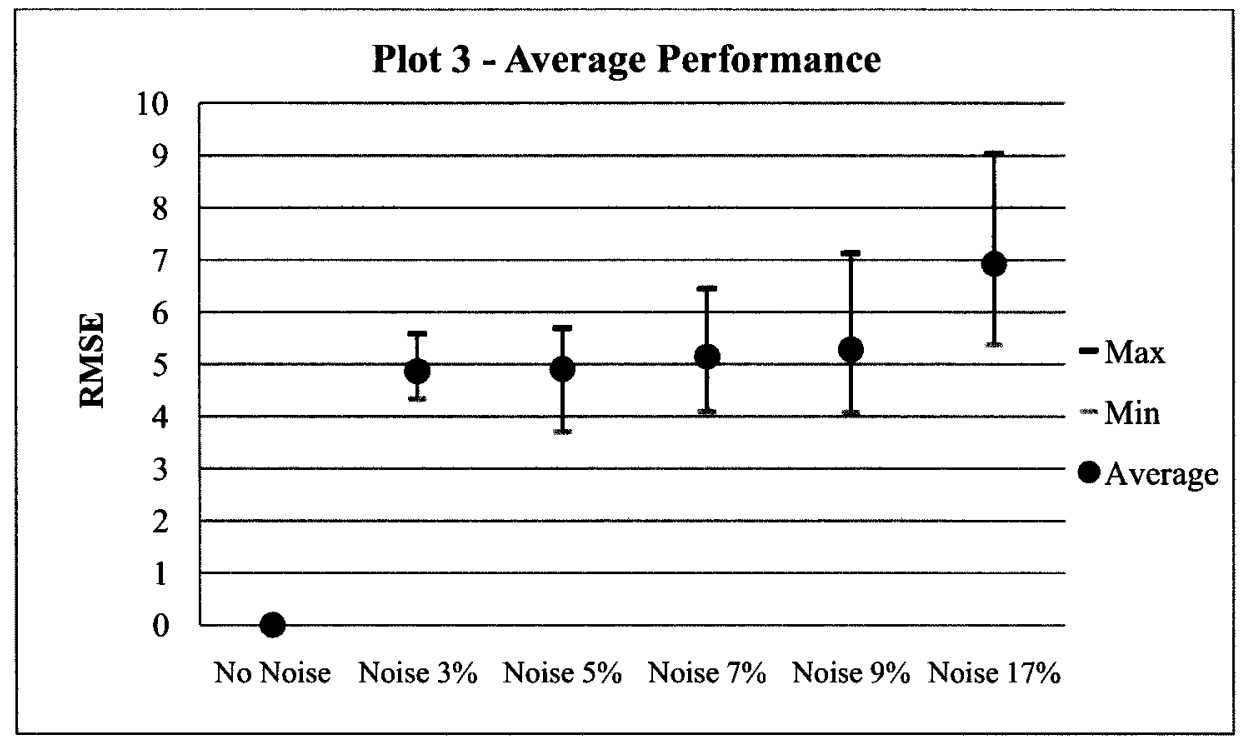

Figure 5.18: Plot 3 - Average performance at each noise level

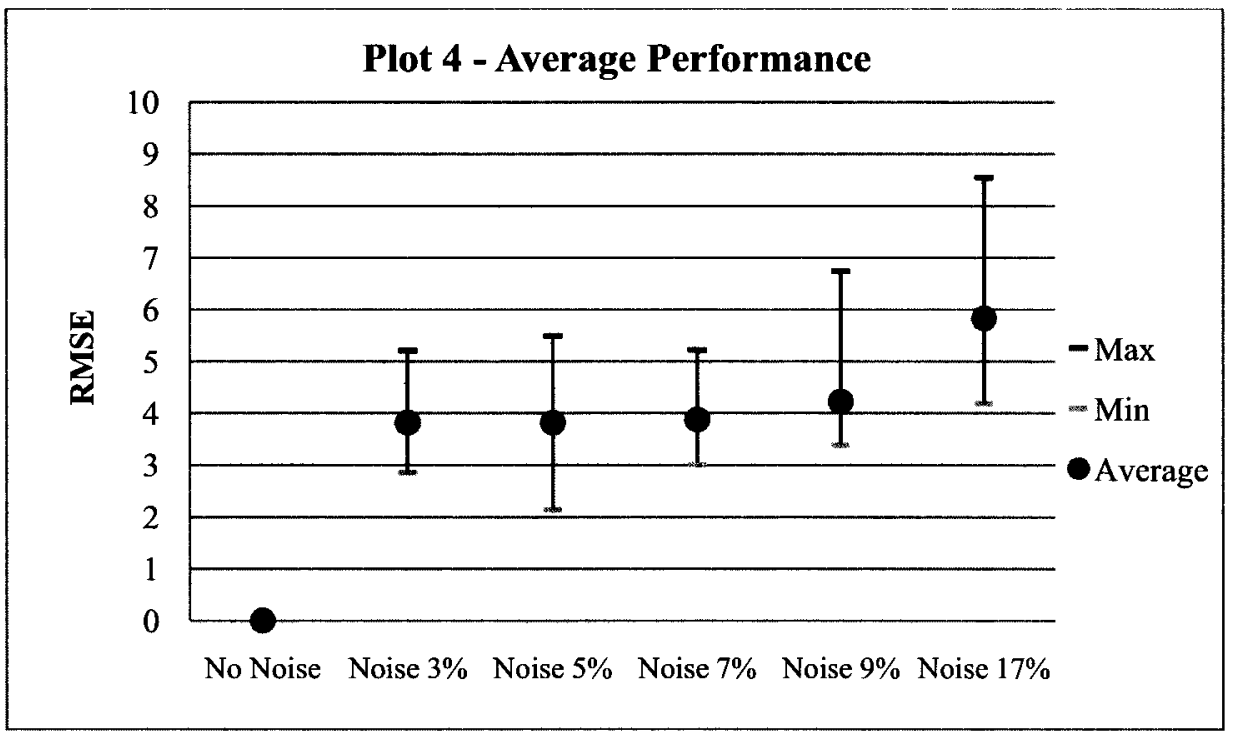

Figure 5.19: Plot 4 - Average performance at each noise level 


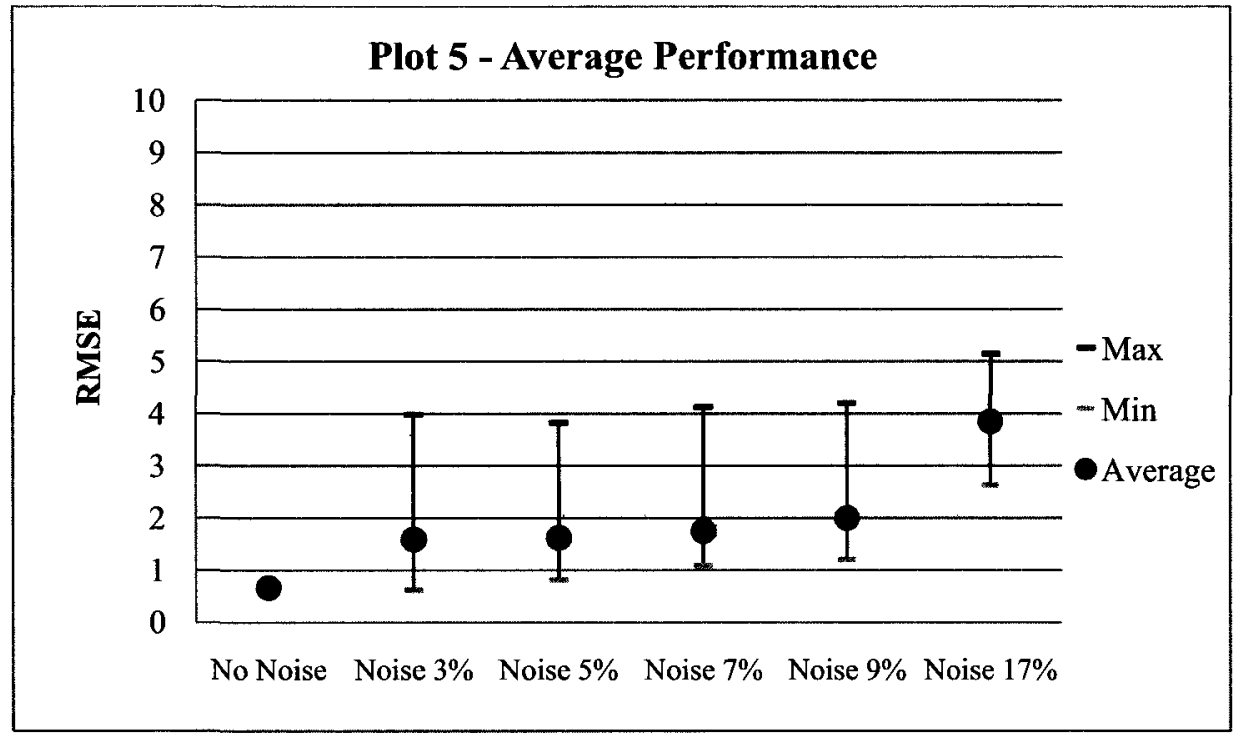

Figure 5.20: Plot 5 - Average performance at each noise level

The aggregate average results (Figure 5.21) help illustrate the collective performance of all plots for the segmentation algorithm. From these results, it can be noted that for Plot 3 and 4, the accuracy of the segmentation algorithm is greatly affected by the addition of noise in detecting underlying trends. This can be seen by the large increase in the RMSE value from the 'No Noise' values around 0 up to values around 5 and 4 ('Noise 3\%') for Plot 3 and 4 respectively. The other plots are able to handle the additional noise more successfully with less range in the results from 'No Noise' to 'Noise 17\%'. 


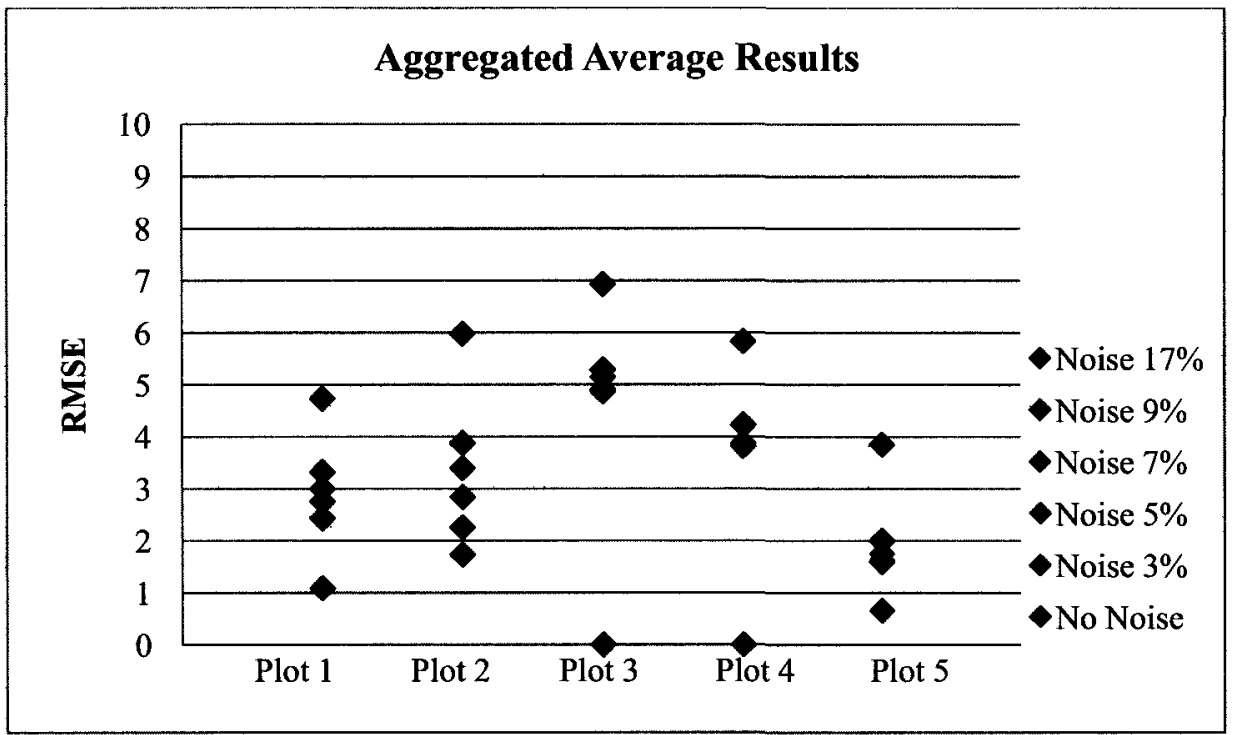

Figure 5.21: Aggregate average results

\subsubsection{Simulation Results - Establishing Threshold Values}

Plots 1 through 5 were created as example data trends to demonstrate the capabilities of the algorithm to handle varying levels of noise added to the signal but they by no means encompass all forms of trends. Data trends may exist is several other different fashions such as the frequency of the cyclical component feature. An example of this is the difference between Plot 1 which is a $\sin \left(\frac{x}{2}\right)$ curve and Plot 2 which is a $\sin \left(\frac{x}{4}\right)$ curve. Both plots exhibit similar primitives but at different frequencies.

The algorithm's threshold values greatly affect the algorithm's ability to detect underlying trends. If these values are set for one type of data trend they may not be able to accurately detect others. Therefore, it was deemed important to verify whether it was best to establish these values with lower frequency or higher frequency data trends.

The algorithm's threshold values were optimized for Plot 2 , a $\sin \left(\frac{x}{2}\right)$ curve. The simulation exhibited similar RMSE results for varying values of $t h_{2}$ and thus all three 
combinations were considered. These three combinations of threshold values were then used for trend detection on curves from $\sin \left(\frac{x}{3}\right)$ to $\sin \left(\frac{x}{9}\right)$ and the RMSE values calculated. The results are displayed in Figure 5.22.

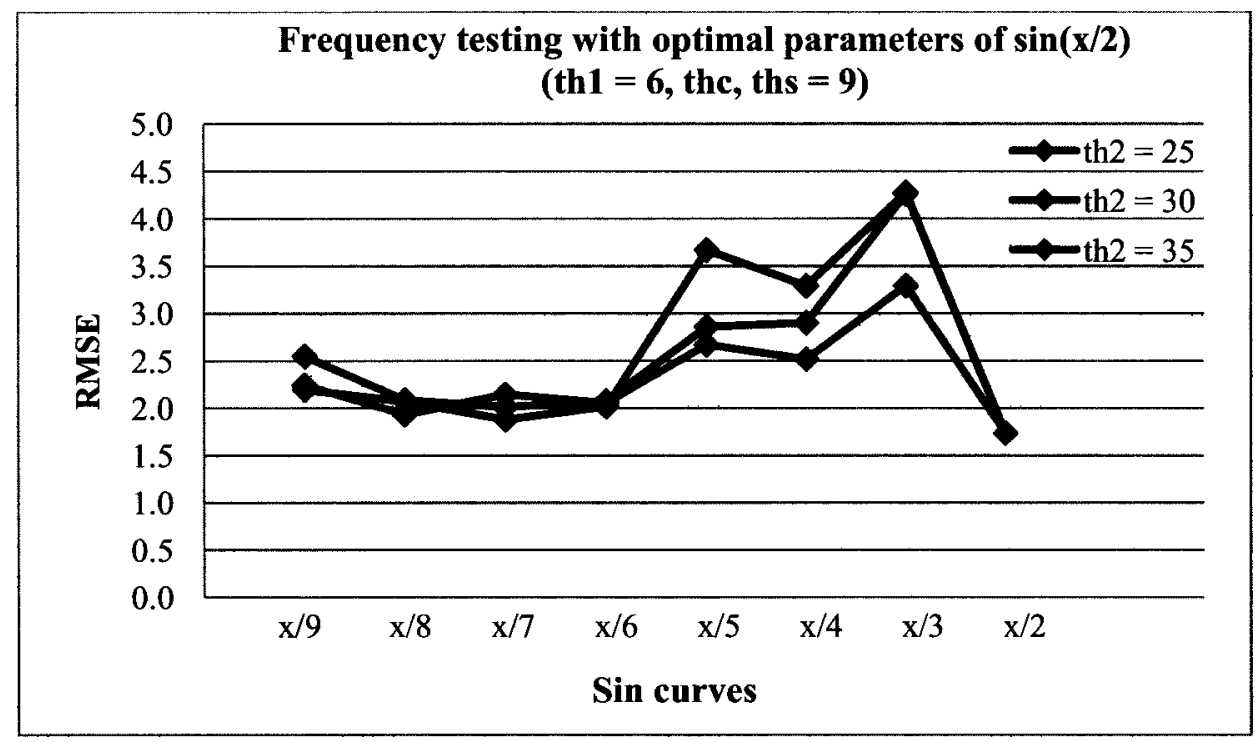

Figure 5.22: Frequency testing with optimal parameters of $\sin (x / 2)$

The algorithm's threshold values were then optimized for a $\sin \left(\frac{x}{9}\right)$ curve. Same as above, similar RMSE results occurred for varying values of $t_{2}$ and thus all three combinations were considered. These combinations of threshold values were then used for trend detection on curves from $\sin \left(\frac{x}{2}\right)$ to $\sin \left(\frac{x}{8}\right)$. The results are displayed in Figure 5.23. 


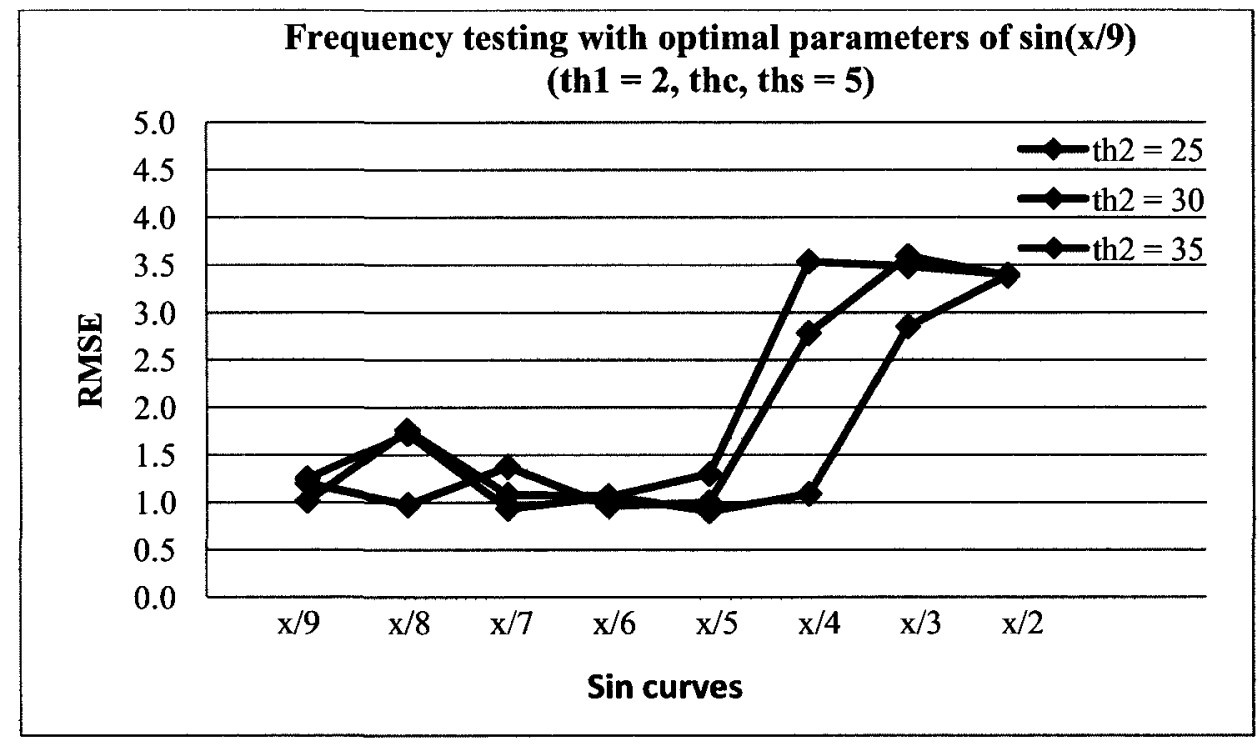

Figure 5.23: Frequency testing with optimal parameters of $\sin (x / 9)$

Extracting the results of the best combination of threshold values from Figure 5.22 and Figure 5.23, the two methods can be compared in Figure 5.24. The results establish that it is best to optimize to lower frequency data trends (given the data trends in this research) because the higher frequency trends will still be accurately, if not more accurately, detected.

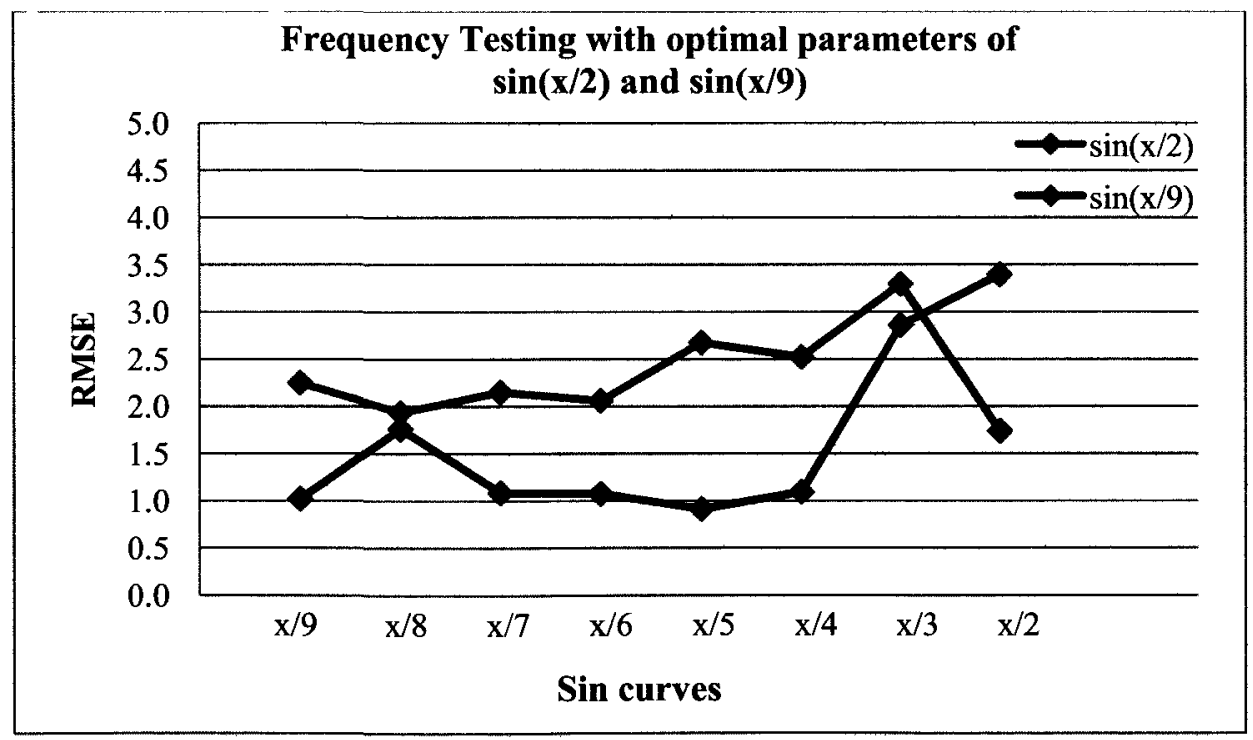

Figure 5.24: Frequency testing with optimal parameters of $\sin (x / 2)$ and $\sin (x / 9)$ 


\subsubsection{Summary}

There are a number of advantages of this technique for trend detection. This technique does not require any pre-filtering of the data because the algorithm performs signal filtering [131] and [133]. In filtering the signal in this manner, the algorithm does not distort steep changes like a traditional low-pass filter would. The algorithm is capable of doing this because consecutive segments may be discontinuous. Classifying the signal into discontinuous shapes (e.g. Step or Transient) by detecting large signal discontinuities, permits the modeling of sudden and large increases/ decreases of the signal [131]. These types of changes could represent an emergent situation for a home care client and the detection of these changes allows for earlier response to a potential crisis and/ or preventive actions to be taken.

A disadvantage of this approach for trend detection is the predefinition of the expected normal behaviours of the parameter being monitored and the usage of fixed value thresholds. The thresholds cannot always take into account the different degrees of the parameter's abnormalities. In the field of home care, it is difficult to define such static trajectories of the observed parameters in advance. Varying expected normal behaviours are possible and depend on the degrees of the parameter's abnormalities. These normal expectations deviate according to the home care client's condition in the past. "Therefore, these thresholds need to be dynamically derived during the observation period" [136]. This method is somewhat similar to the process described above in selecting the optimal parameters based on the un-noisy signals. Nevertheless, an adaptive, on-line version of the algorithm is more ideal and has been explored in [65]. Implementing the algorithm presented in [65] was beyond the scope of this research. 
In this research, using the segmentation algorithm for detecting trends demonstrated three important traits. The algorithm was able to fairly accurately detect trends with no noise but had a difficult time with some data trend types with the addition of noise. The algorithm was also able to more accurately detect data trends when optimized for lower frequencies of the particular data trend to be detected.

\subsection{Detecting Trends Using a Neural Network}

As introduced in sub-section 2.2 .3 , there are a variety of features of neural networks. For example, there are three fundamentally different classes of network architectures: singlelayer feed-forward, multilayer feed-forward, and recurrent networks. There are also two learning processes through which neural networks function: learning with a teacher, also referred to as supervised learning, and learning without a teacher, which contains two subcategories; reinforcement and unsupervised learning. There are also a number of learning tasks and activation functions [98].

In determining a neural network for use in this research, the goal to consider was to display a similar output to a clinician as the segmentation algorithm. Therefore, the output had to contain semi-qualitative episodes of trends as well as a time-series graph of the extracted client's trends. Each semi-qualitative episode should contain one of three types of primitives, \{Increasing, Decreasing and Steady\}, the starting and end points, and their corresponding magnitude.

Two classes of network architectures were explored for the purpose of retrospectively analyzing a home care client's data for trends. These two types are discussed below. 


\subsubsection{Recurrent Neural Networks}

Within this type of neural network architecture, there are a number of slightly different variations. One of these is the Elman type recurrent neural network $(\mathrm{RNN})$. In principle, this type of RNN is set up as a regular feed-forward network. This suggests that all neurons in one layer are connected with all neurons in the next layer. Where this architecture differs is its so-called context layer which is a special case of a hidden layer. The neurons in this layer hold a copy of the output of the hidden neurons. This output is copied into a specific neuron in the context layer. The value of the context neuron can then be used one time step later as an additional input signal for all the neurons in the hidden layer. This implies that the Elman network has a memory of one time lag [137] and "this memory facilitates the learning of time-sequential patterns" [138].

Simply put, the two main types of RNNs can be roughly classified as follows [139]:

- Jordon type network: the feedback data from output layer are fed to the input layer as a part of input data in the layered structure neural network

- Elman type network: the feedback data from hidden layer are fed to the input layer as a part of input data in the layered structural network

- The feedback data are fed to the same layer in the layered structural neural network or arbitrary mutual uniting neural networks

Data from the hidden layers is incorporated with the current input data from the outside. This feedback information allows the trend of the time series data to be continuously taken into account. The number of units in the input layer is determined from the number of observable parameters and feedback lines. The number of units in the 
output layer is determined by the number of trends or characteristics to be detected. The number of units in each hidden layer and the number of hidden layers are directed by the complexity of the problem at hand. The two main points to bear in mind when designing a network is the generalization ability, discussed in more detail in the following section, and the training speed. Although, as long as the network is not utilized online, the training speed is not as significant [139].

Unfortunately, the use of one time lag does not lend itself well for mining lengthy trends retrospectively. It also presents a difficult situation for creating target sets for a given input training set if the input contains slow trends.

Where this type of network excels and has been used extensively is in prediction systems and for the detection of faults in industry environments [138]-[140]. In the case of home care monitoring, the ability to detect in advance the degradation of a client's health is crucial since it can lead to an urgent situation.

\subsubsection{Feed-forward Neural Networks}

The feed-forward neural network was the second type of network architecture explored. This type of network has previously shown the ability to capture important information in process trends in [86], [87]. A back propagation-learning algorithm was considered for use in this research, similar to that in Rangaswamy and Venkatasubramanian [87], because of its ability for pattern recognition. Training a network by back-propagation involves three steps as outlined by [141], these include:

1. Feed-forward of the input training pattern

2. Back-propagation of the associated error

3. Adjustment of weight 
A sigmoid activation function was considered for the network because it is "highly nonlinear and has been reported to have a good performance when working with the back-propagation learning algorithm" [142]. Other features of the network such as the back-propagation network training function and the weight/ bias learning function were chosen by the default settings of MATLAB. These two functions were the Levenberg-Marquardt back-propagation and gradient descent with momentum weight and bias learning function. The network architecture, consisting of input, hidden and output layers, used to detect the primitives introduced in sub-section 5.2.2 is shown in Figure 5.25 .

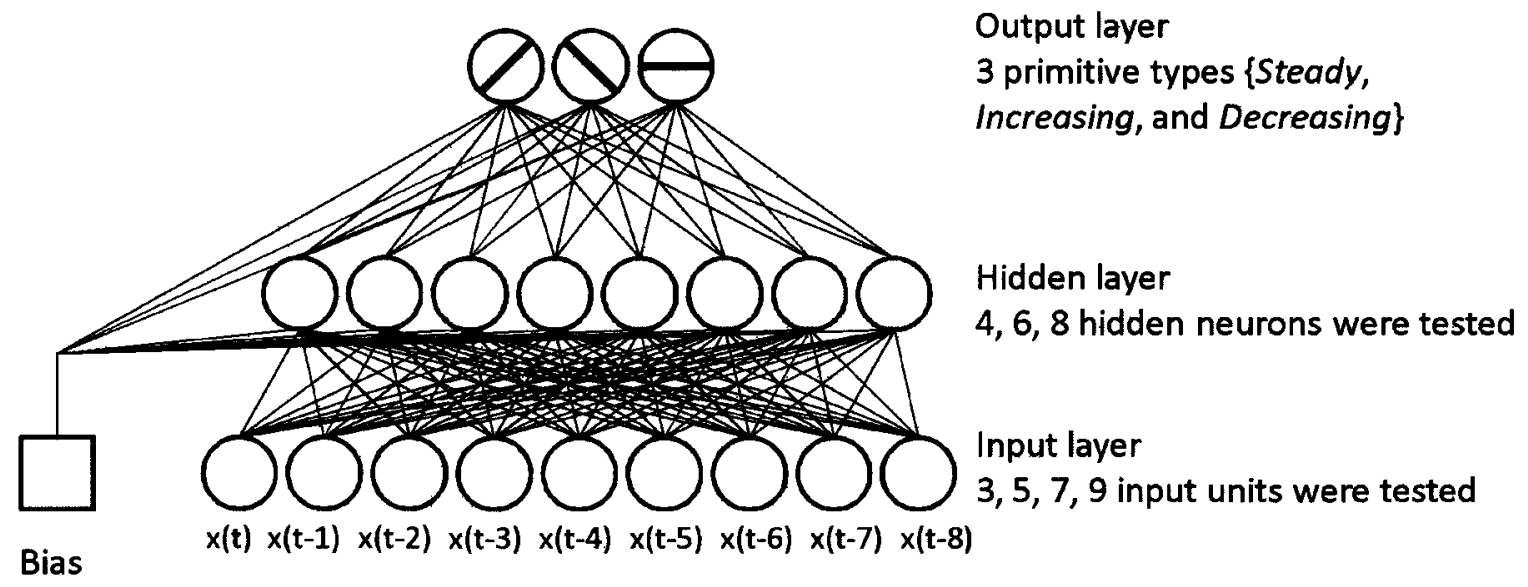

Figure 5.25: Feed-forward neural network architecture

As previously stated, the results from the neural network had to be similar in nature to that of the segmentation algorithm. Therefore, the network architecture only consisted of three primitives in the output layer. In order to successfully classify the three primitives, two main stages required careful attention [41]:

- Learning: The developed model must represent an adequate fit of the training data. The data itself must contain the relationships that the neural network is 
trying to learn, and the neural network model must be able to derive the appropriate weights to represent these relationships.

- Generalization: The developed model must also perform well when tested on new data to ensure that it has not simply memorized the training data characteristics. It is very easy for a neural network model to "over-fit" the training data, especially for small data sets. The architecture needs to be kept small, and key validation procedures need to be adopted to ensure that the learning can be generalized.

In order to correctly detect the three primitives of interest, training sets which included all three were created. Fifteen percent of the samples were also set aside for validation and another $15 \%$ for testing. The targets for each of the training inputs were constructed based on the first derivative of the input units. For example, in Figure 5.25, the network has input units labelled as $x(t), x(t-1) \ldots x(t-8)$. Therefore, the derivative of the input units at varying sizes would be calculated and the step would be repeated for the entire length of the input data. The derivate determines which primitive best represents the data in each input window. A positive first derivative represents an Increasing primitive, a negative derivative represents a Decreasing primitive, and a zero derivative represents a Steady primitive. This procedure corresponds to the first step outlined by Rangaswamy and Venkatasubramanian [87] for the detections of data trends. Following this step, the procedure of aggregating primitives is very similar to that of the segmentation algorithm discussed in sub-section 5.3.1; this process includes the steps listed below:

1. Assignment of the primitives to each time interval

2. Combination of primitives to generate episodes 
3. Sequencing of the episodes with the corresponding interval length to develop the trend

4. Generation of an overall structure (i.e. the profile). The data structure for the profile carries the following information: primitive associated with episode, interval length, initial and final values and pointer to the next profile structure.

In processing the steps similarly to the segmentation algorithm, it must be noted that any Steady episode's end points have to be augmented in order to display them as flat segments on a display. A Steady episode's end points were calculated as described above in sub-section 5.3.1 as the median of all the points included in the episode. Correspondingly, the end points in the episodes beside any Steady episode also had to change so that they could line up with one another.

Rangaswamy and Venkatasubramanian [87] put emphasis in normalizing the input data so that "problems one might face due to the actual magnitudes of the sensor trends" [87] can be removed. It was chosen not to do this process in the case of this research because if the neural network is to be used online, one cannot forecast the minimum and maximum values of the data which are needed in the calculation of the normalization scheme.

\subsubsection{Simulation Results - Noisy Data}

In order to properly mine the data trends introduced in sub-section 5.2.2, a method, comparable to that of the segmentation algorithm, to select the appropriate values of the neural network's feature had to be undertaken. The features to be modulated are the following:

1. Number of input units 


\section{Number of hidden neurons}

There are other additional parameters that can be changed in the neural network, such as learning functions, but these were not modulated because they have shown to work well for trend detection [87]. Another parameter that can be changed is the number of hidden layers in a neural network. Neural networks can have a number of hidden layers, however in practice, incorporating more than two is rarely done [56]. Therefore, it was deemed sufficient to simply test a neural network with one hidden layer.

Deciding the number of input units for the neural network was also guided by [87] whom detected trends with a window of five samples. For that reason, various window sizes around five samples were evaluated as shown in Table 5.6.

The second parameter that was modulated was the number of hidden neurons. Deciding this value is very important part of the overall network architecture. As shown in Figure 5.25, the hidden layer does not directly interact with the external environment but does have a great influence on the final output. Using too few neurons in the hidden layers results in under-fitting; the neurons cannot adequately detect the signals in a complicated data set. Alternatively, using too many neurons results in several problems. One of these problems is over-fitting. As defined by [143], "over-fitting occurs when the neural network has so much information processing capacity that the limited amount of information contained in the training set is not enough to train all of the neurons in the hidden layer". Another problem that too many neurons in the hidden layer cause is an increase in training time.

One issue discovered that was not outlined in [87] was that a home care client's data would very rarely produce an episode with a derivative of zero. Thus, a range 
around zero could also be determined such that most segments would not simply be Increasing or Decreasing.

Thus, using the original data sets from Figure 5.5 as the gold standard for the model to match, the optimal values for the features can be selected by testing $x^{n}$ combinations of values where, $x$ equals the number of values tested (3) and $n$ equals the number of features (2). The combination of values with the overall smallest combined RMSE was selected (Table 5.6). As stated previously, the values of the tuning parameters are not suitable for all cases.

Table 5.6: Testing and final values of the tuning parameters for the simulated data sets

\begin{tabular}{|l|c|}
\hline \multicolumn{1}{|c|}{ Tuning Parameter } & Testing Values \\
\hline Number of input units & $5,7,9$ \\
\hline Number of hidden neurons & $4,6,8$ \\
\hline
\end{tabular}

An example of the result obtained with the optimal combination of values for the tuning parameters on one of the data sets is shown in Figure 5.26. Results for the other plots can be found in Appendix D. 

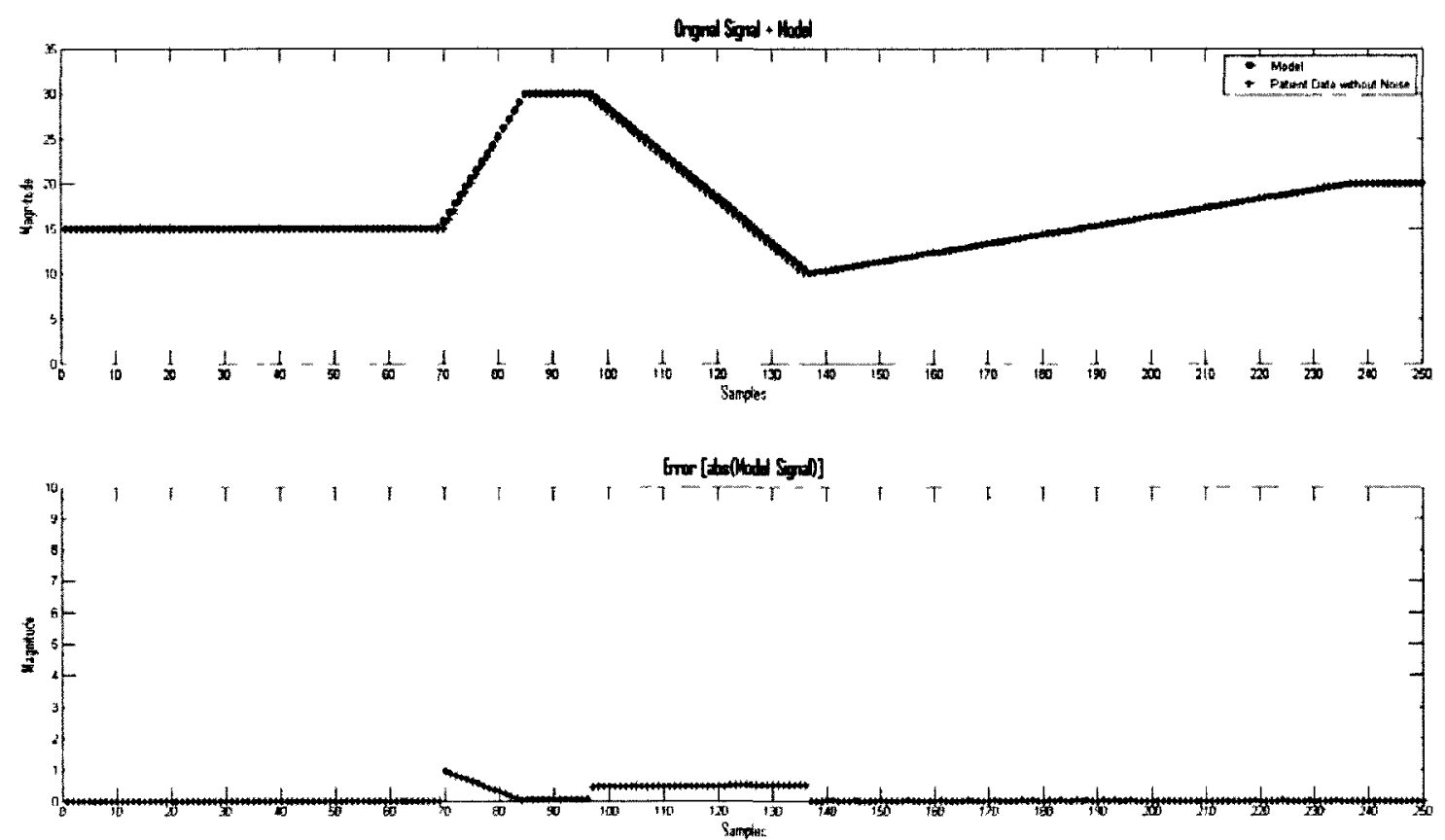

Figure 5.26: Simulated data, Plot 5, and the corresponding output from the feed-forward neural network

Once the optimal combination of values for the tuning parameters was discovered, the neural network could be presented with the varying noise levels on each trend type. Since the Gaussian noise added to each trend type was generated by a random number generator, it was important to perform a number of iterations for this step in order to obtain an accurate representation of the network's performance. Testing the network one hundred times with each noise level was completed in order to demonstrate this concept. An example of each noise level with Plot 5 is shown from Figure 5.27 to Figure 5.31. 

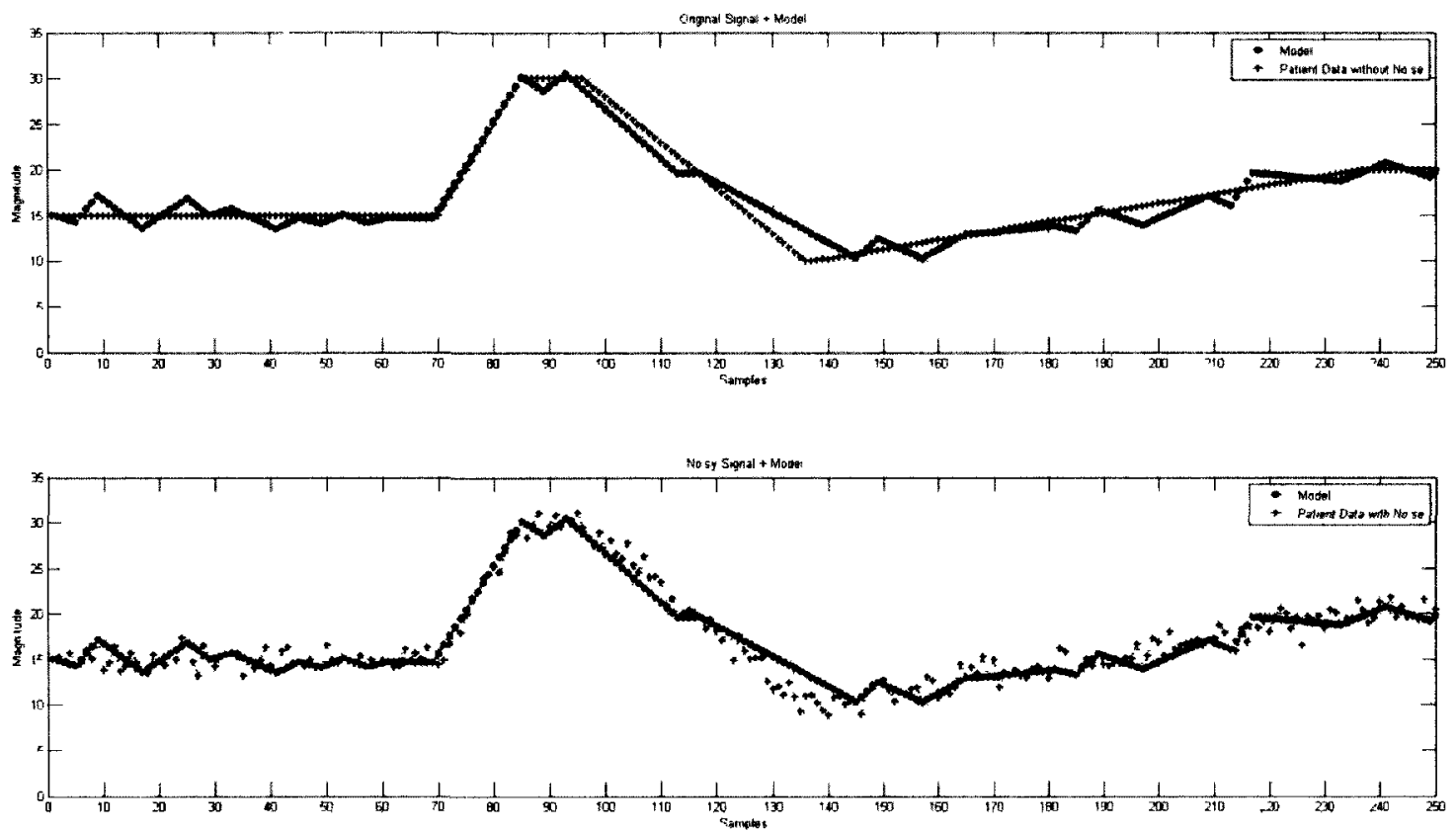

Figure 5.27: Plot 5 - 3\% noise
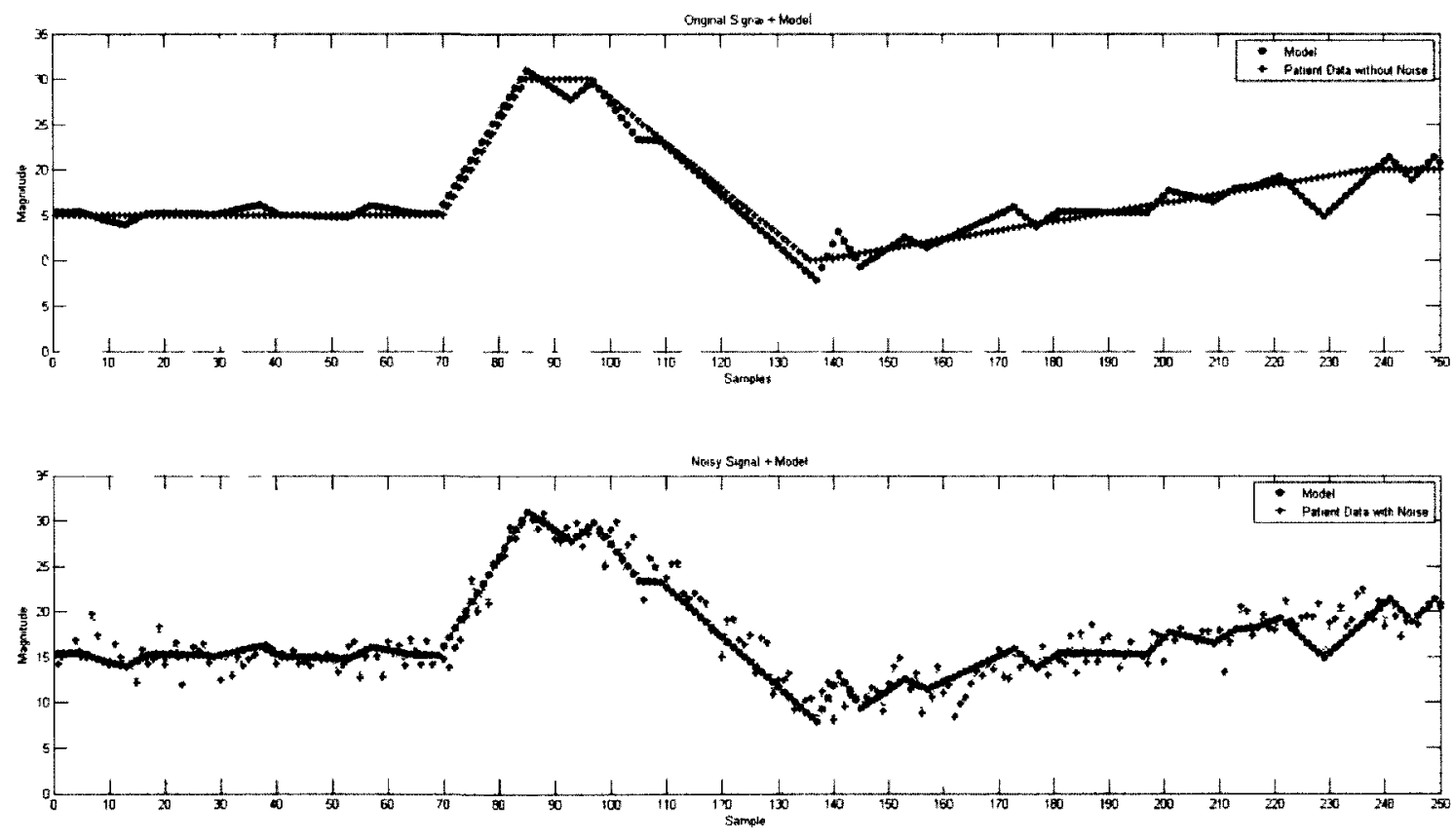

Figure 5.28: Plot 5 - 5\% noise 

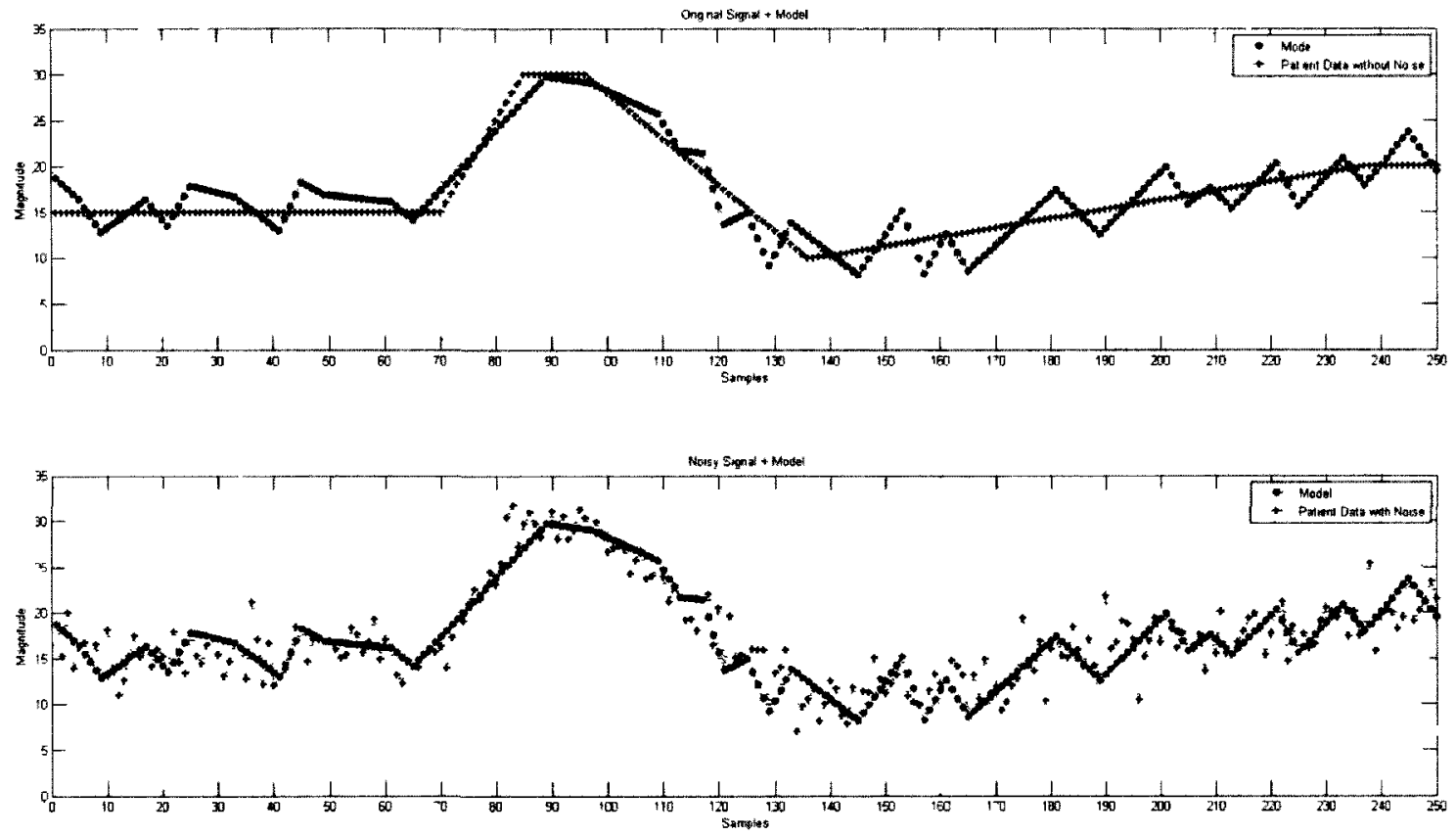

Figure 5.29: Plot 5 - 7\% noise
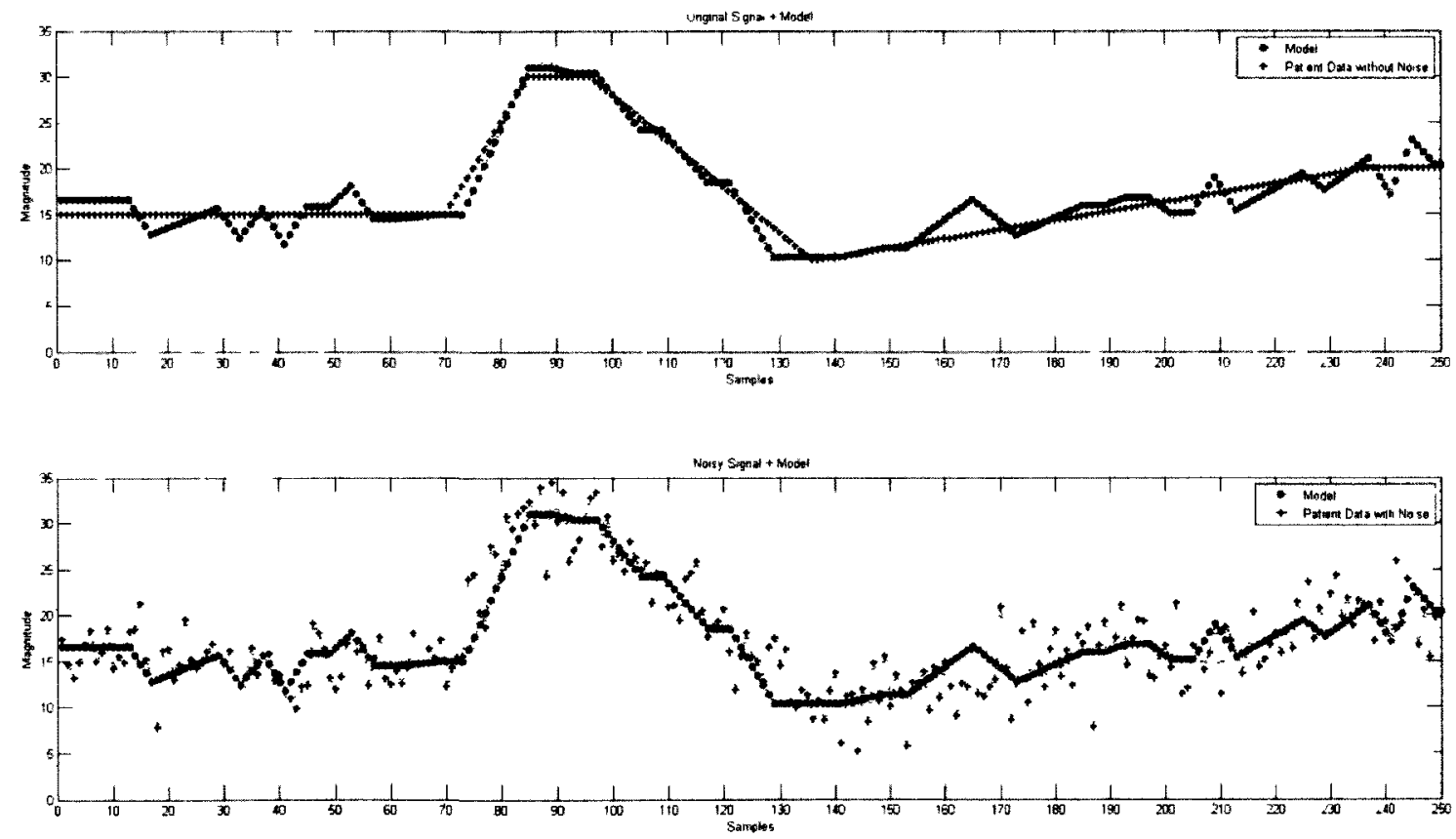

Figure 5.30: Plot 5 - 9\% noise 

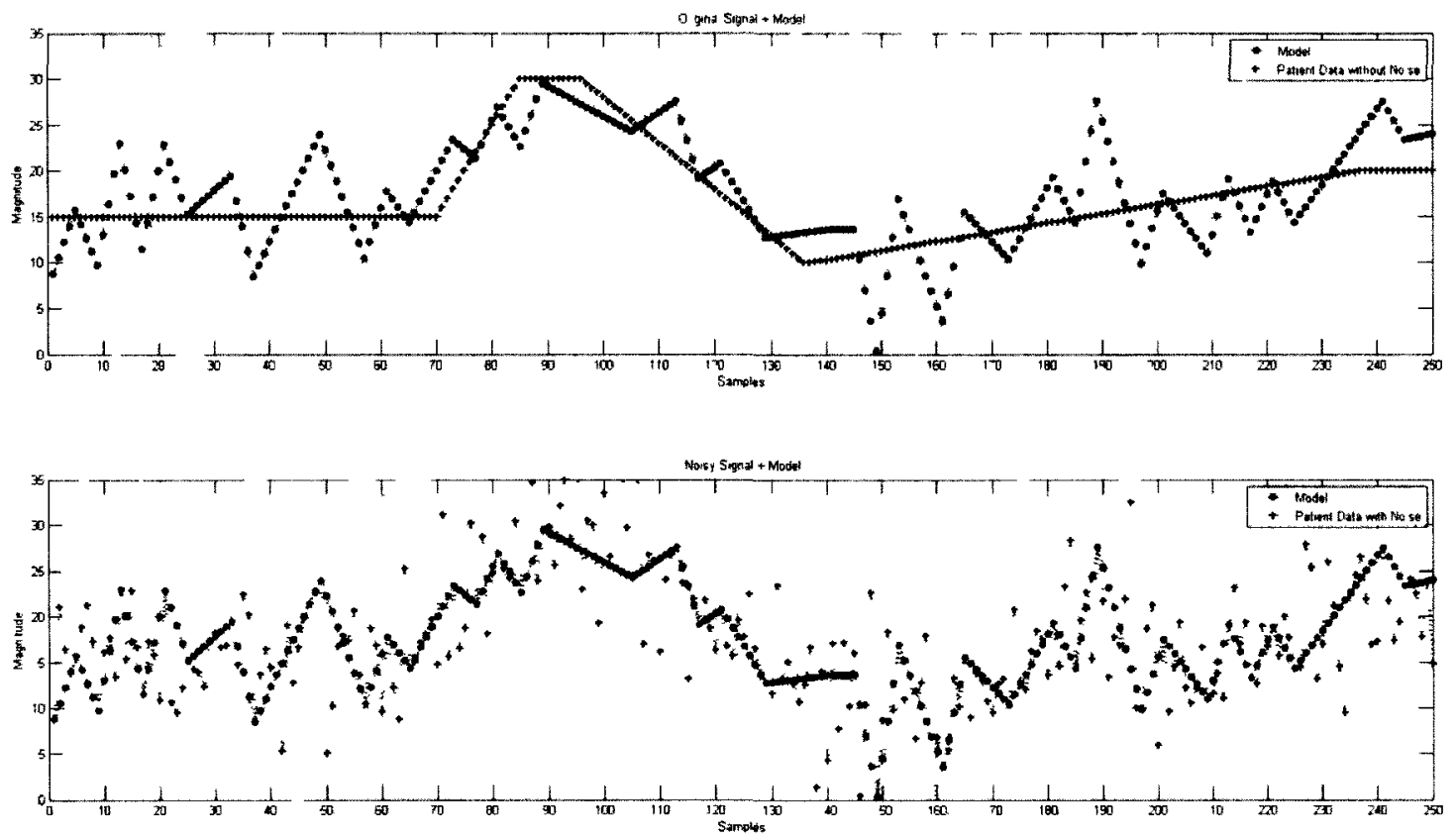

Figure 5.31: Plot 5 - 17\% noise

The performance of the data mining technique at each noise level and for each trend type can be determined by the value of root mean squared error. This value was averaged over the 100 passes for each trend type and the results are shown in Figure 5.32 to Figure 5.36.

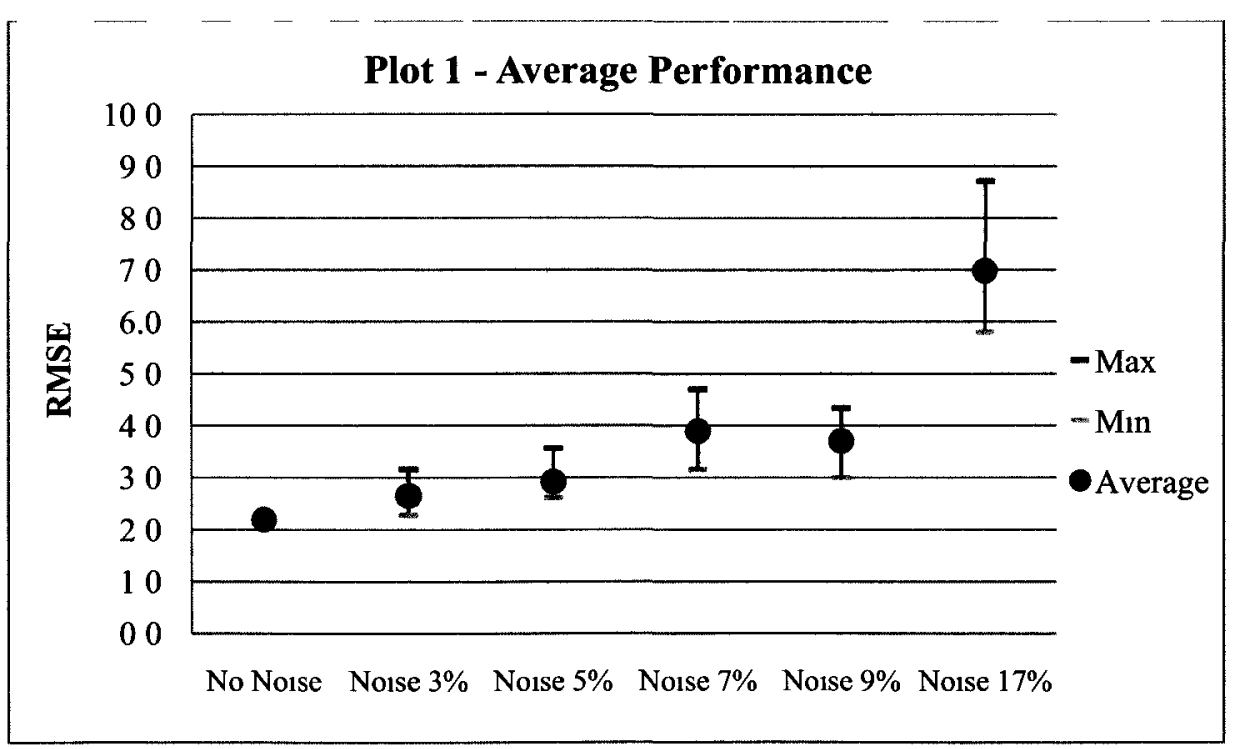


Figure 5.32: Plot 1 - Average performance at each noise level

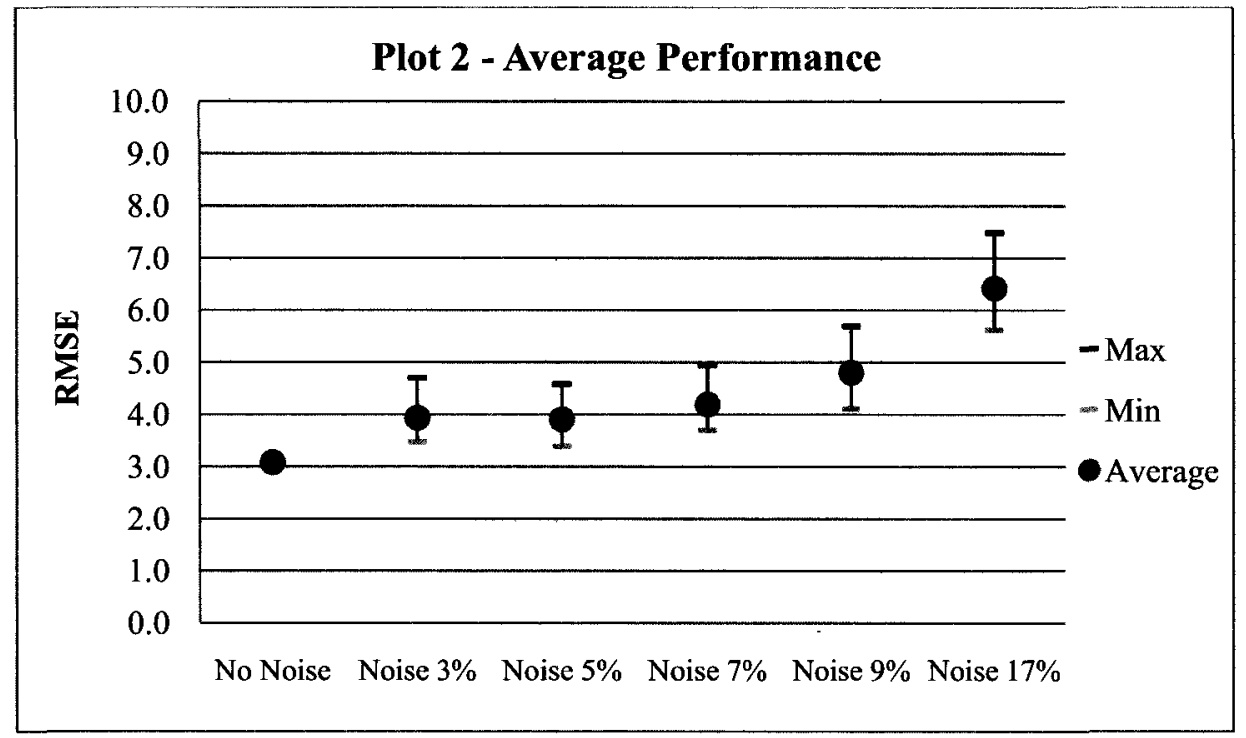

Figure 5.33: Plot 2 - Average performance at each noise level

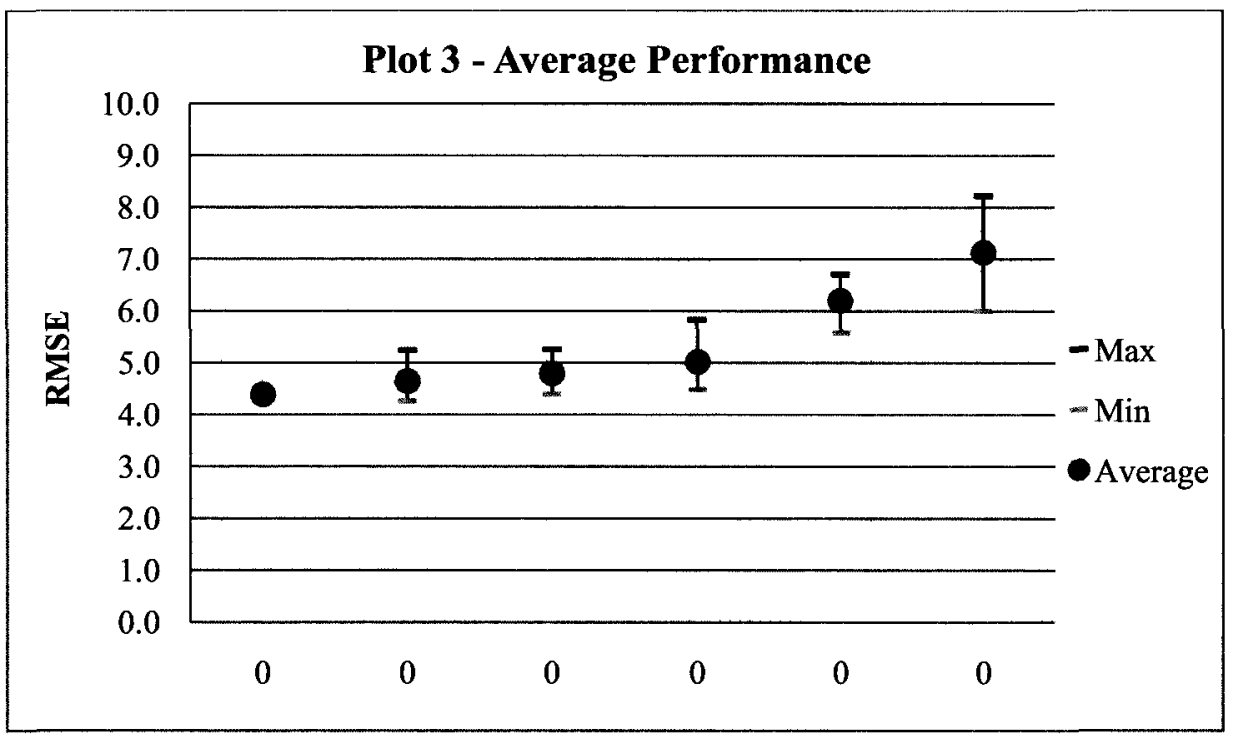

Figure 5.34: Plot 3 - Average performance at each noise level 


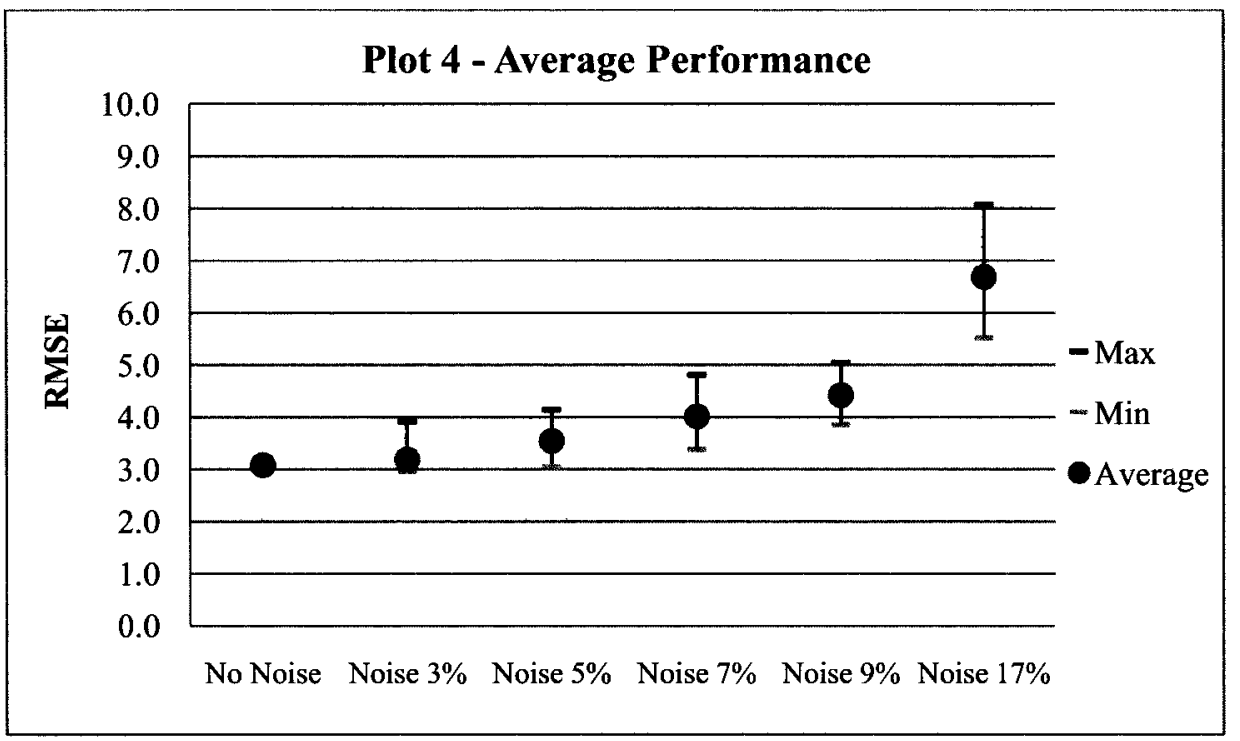

Figure 5.35: Plot 4 - Averaged performance at each noise level

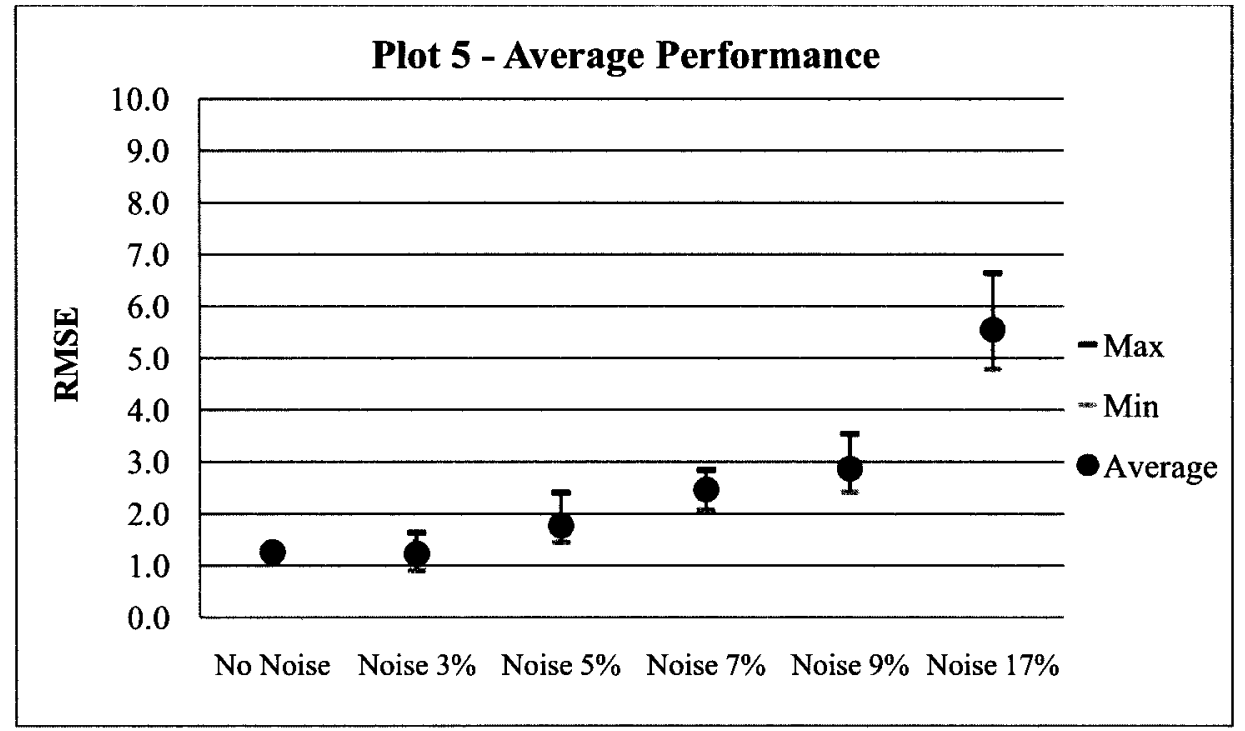

Figure 5.36: Plot 5 - Average performance at each noise level

The aggregate average results (Figure 5.37) help illustrate the collective performance of all plots for the neural network. From these results, it can be noted that the neural network is able to handle the addition of varying levels of noise fairly well for the given data trends. This can be seen by the small spread is the results (e.g. the largest span from 'No Noise' to 'Noise $17 \%$ ' is 4.8 ). 


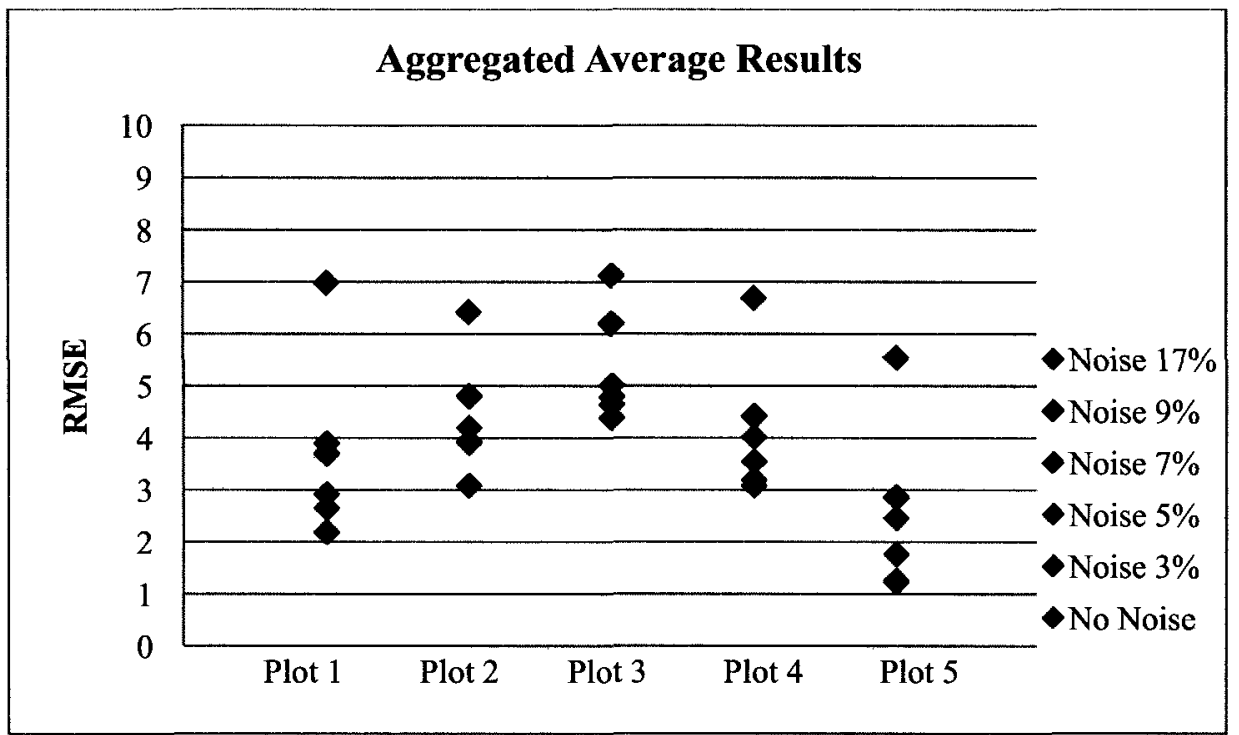

Figure 5.37: Combined average performance at each noise level for all plots

Incorporating more than the 3 original primitives Increasing, Decreasing, and Steady\} to include: \{Concave Up, Concave Down, Convex Up, and Convex Down permits the neural network to more accurately represent data trends. For example, Concave Up (respectively Concave Down), permits the neural network to convey information to a clinician that a client's health data is beginning to steady (Figure 5.38). The other example in Figure 5.38 demonstrates the ability of Convex $U p$ and Convex Down (respectively Concave Up and Concave Down) to better represent transients in the data without flattening or over-extending the minimum (respectively maximum) segment. 


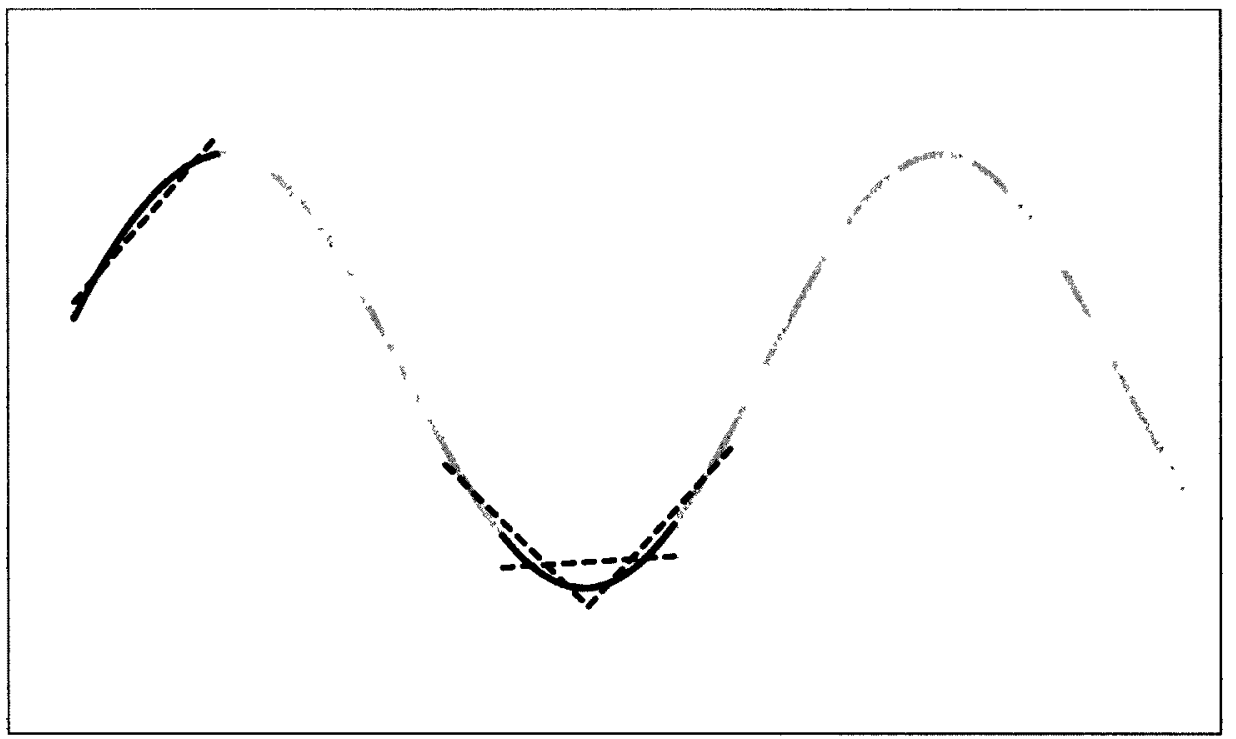

Figure 5.38: Two scenarios demonstrating the advantages of the additional primitives

An example of the output for Plot 1 using a neural network that can detect seven primitives is shown in Figure 5.39. These additional primitives were detected by calculating the second derivative of the input units in the training set. The results for Plot 2 - Plot 5 with the optimal parameter values of the neural network with seven primitives can be seen in Appendix F. 

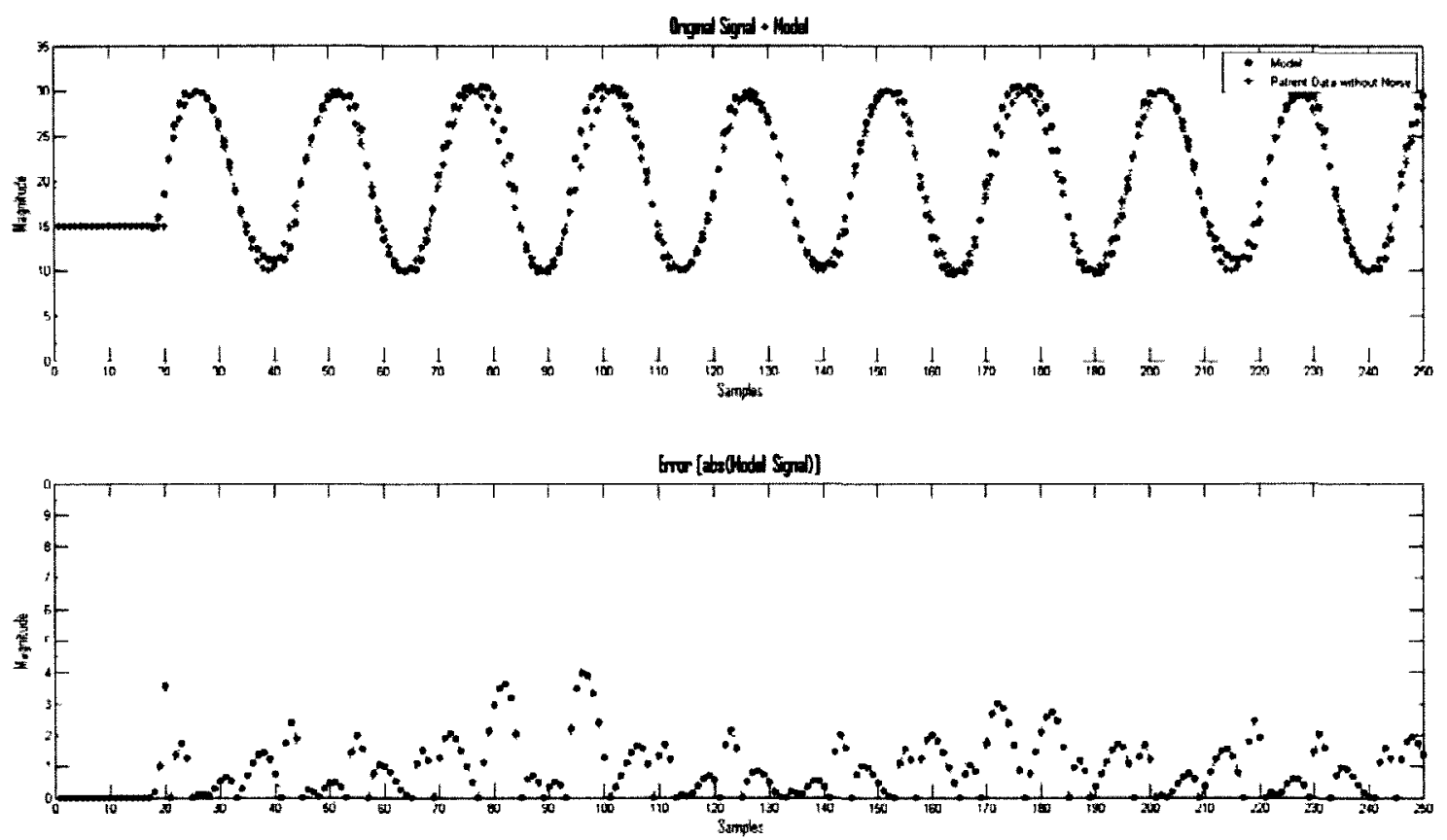

Figure 5.39: Simulated data, Plot 1, and the corresponding output from the feed-forward neural network using 7 primitives

Using the optimal parameter values, the RMSE values for the neural network with three primitives and with seven primitives can be compared (Figure 5.40). The results from this research indicate that using seven primitives results in similar or lower RMSE values for the data trends. This indicates an area of improvement using the neural network for trend detection. 


\section{Aggregated Average Results}

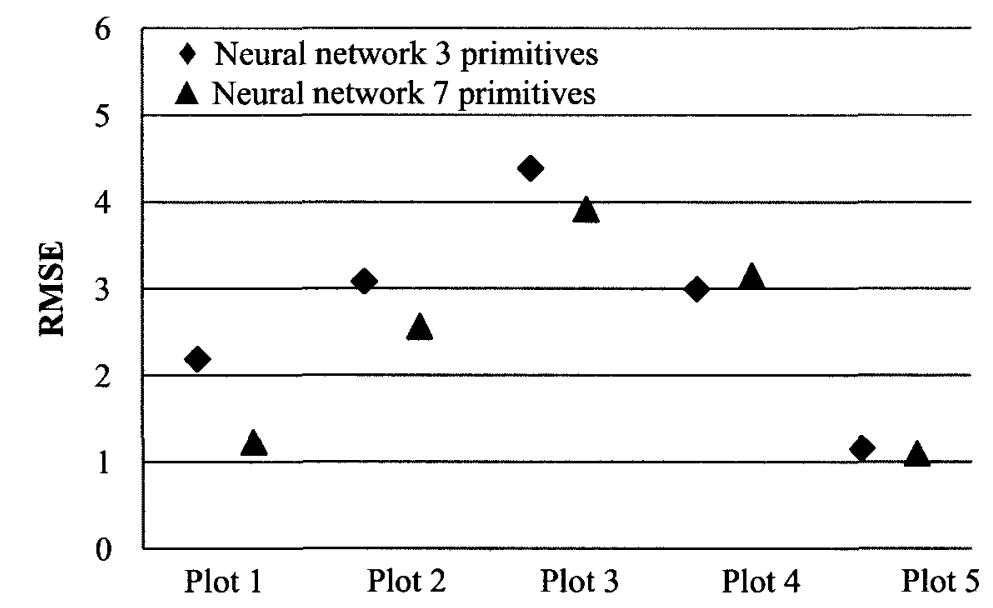

Figure 5.40: Aggregated average results for feed-forward neural network using 3 and 7 primitives

\subsubsection{Simulation Results - Establishing Network Values}

Similar to sub-section 5.3.3, the neural network had to be tested at different trend frequencies.

The network's number of input and hidden units greatly affects the network's ability to detect underlying trends. If these values are set for one type of data trend they may not be able to accurately detect others. Therefore, it was deemed important to verify whether it was best to establish these values with lower frequency or higher frequency data trends.

The neural network's numbers of input and hidden units were optimized for Plot 2, a $\sin \left(\frac{x}{2}\right)$ curve. The optimal combination was then used for trend detection on curves from $\sin \left(\frac{x}{3}\right)$ to $\sin \left(\frac{x}{9}\right)$ and the RMSE values calculated. The results are displayed in Figure 5.41. 


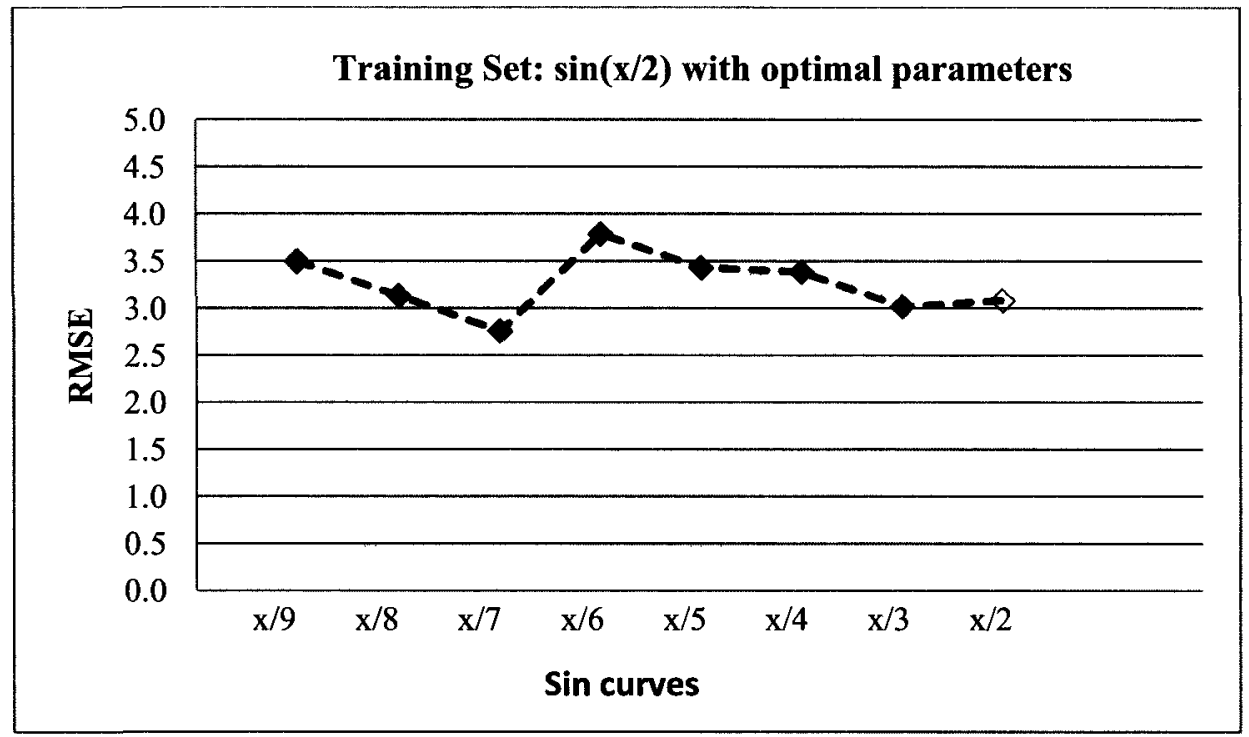

Figure 5.41: Frequency testing with optimal parameters of $\sin (x / 2)$

The algorithm's threshold values were then optimized for a $\sin \left(\frac{x}{9}\right)$ curve. The optimal combination of the number of input and hidden units was then used for trend detection on curves from $\sin \left(\frac{x}{2}\right)$ to $\sin \left(\frac{x}{8}\right)$. The results are displayed in Figure 5.42 .

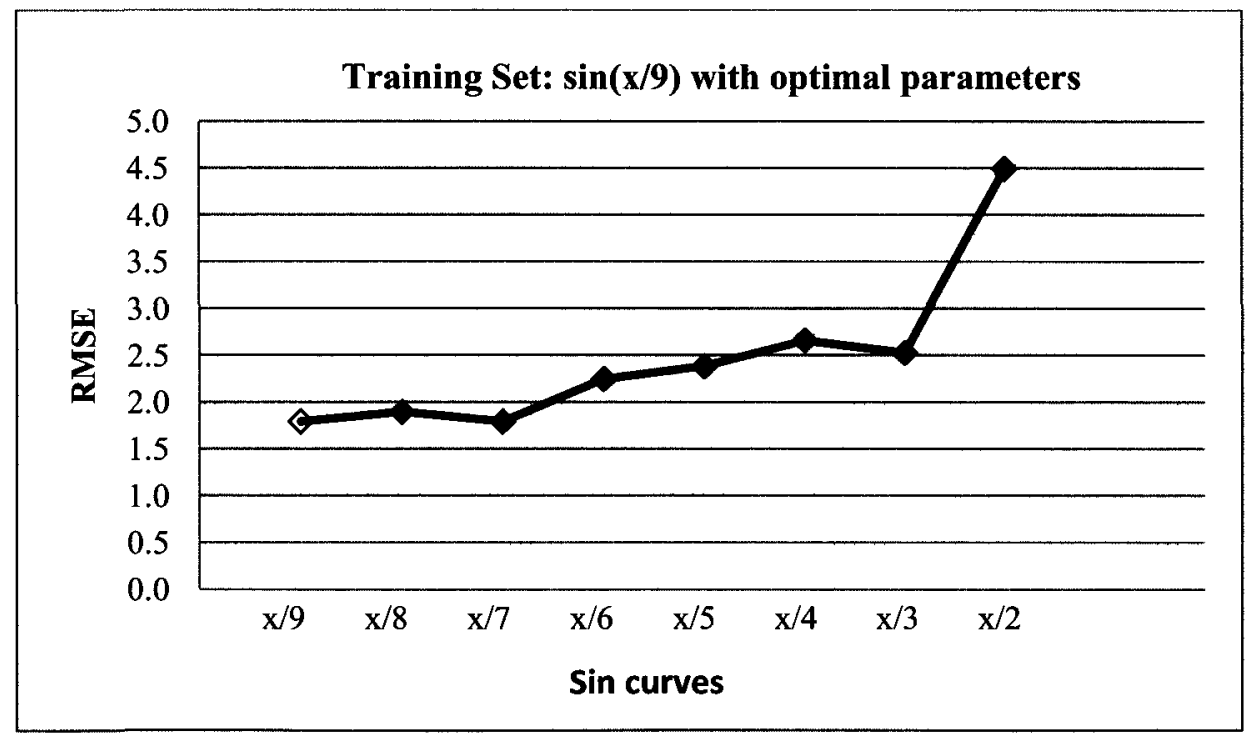

Figure 5.42: Frequency testing with optimal parameters of $\sin (x / 9)$ 
In the case of sin curves, combining the results from Figure 5.41 and Figure 5.42 shows that it is best to optimize to lower frequency data trends because the higher frequency trends will be more accurately detected (Figure 5.43). The testing of a $\sin (x)$ curve was not possible because there were not enough samples in the curve for five input units.

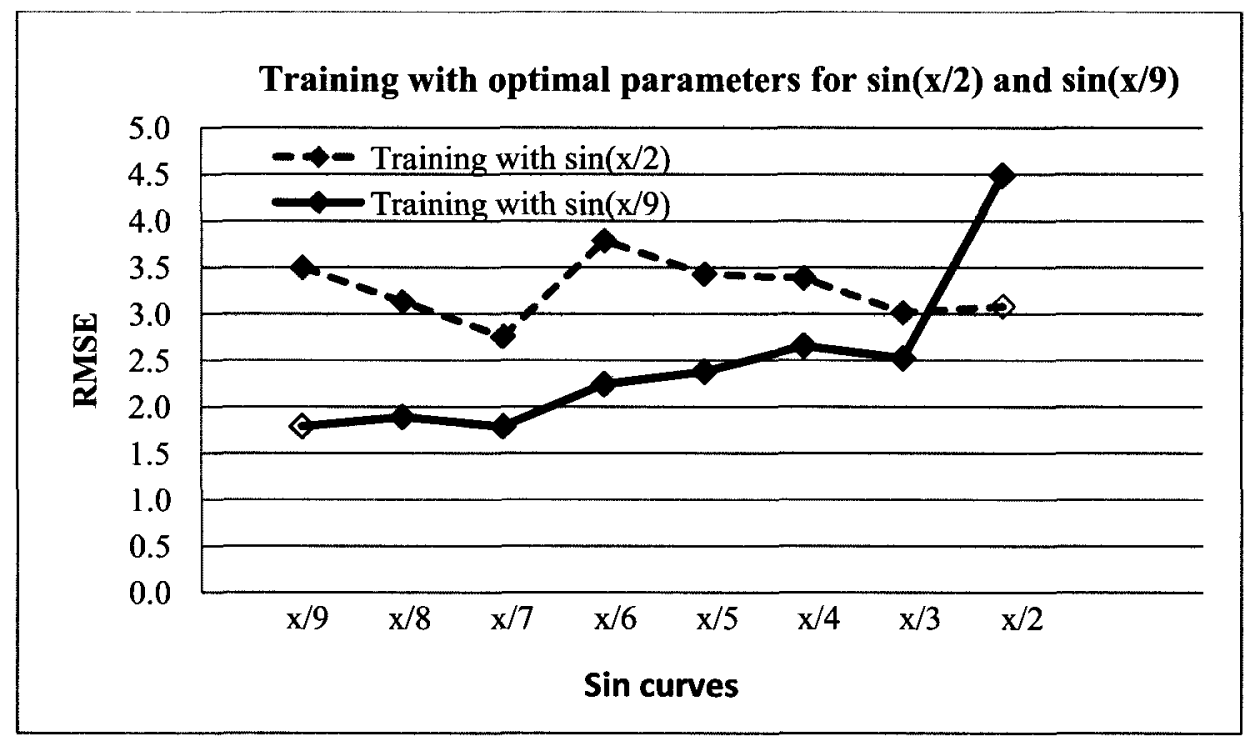

Figure 5.43: Frequency testing with optimal parameters of $\sin (x / 2)$ and $\sin (x / 9)$

\subsubsection{Summary}

An artificial neural network is a potential solution to problems such as the "tedious nature of knowledge acquisition, the inability of the system to learn or dynamically improve its performance and the unpredictability of the system outside its domain of expertise" [87]. Changes to a system which may require retraining of the neural network is simpler than revising a complex knowledge base of an expert system.

Another advantage of using neural networks is that they have demonstrated that they can often outperform other simpler methods because of their ability to represent highly nonlinear function. This characteristic can sometimes result in over-fitting but can 
be resolved by establishing an adequate stopping iteration for the gradient descent in the back propagation algorithm [56].

Neural networks, as opposed to logistic regression, "do not require any assumptions regarding the underlying parameter distributions, nor about the interactions among the independent variables" [56].

A disadvantage of neural network is that the number of hidden neurons per layer that are necessary to effectively learn a desired function are not known in advance [56]. Therefore, several configurations need to be tested in order to find an optimal one as demonstrated in sub-section 5.4.4.

The networks also lack interpretability because the "set of weights is not as understandable as a collection of rules or a decision tree" [56]. Neural networks have no explanation capability and therefore, offer no insight into the problem-solving process [144].

In this research, using a feed-forward neural network for detecting trends demonstrated two important traits. The network demonstrated good capability of handling additional noise to the signals tested and was able to more accurately represent the data trends in this research when trained for lower frequencies of the particular data trend to be detected.

\subsection{Comparison of the Simulation Results}

Analyzing the optimal plots for both data mining techniques, the segmentation algorithm overshoots peaks if there is a steep incline (respectively decline) but consistently follows the increasing (respectively decreasing) segments (see Figure B.2, Appendix B). Alternatively, the neural network truncates peaks (Figure D.2 and D.3, Appendix D) if 
there is a steep incline (respectively decline) and does not consistently follow the increasing (respectively decreasing) segments.

Comparing the results for all plots at all noise levels of the two techniques, the majority (19/25) of the segmentation algorithm RMSE values are lower than the neural network (Figure 5.44). Of the six other plots, the RMSE values for the neural network are only better by $0.1-0.6$.

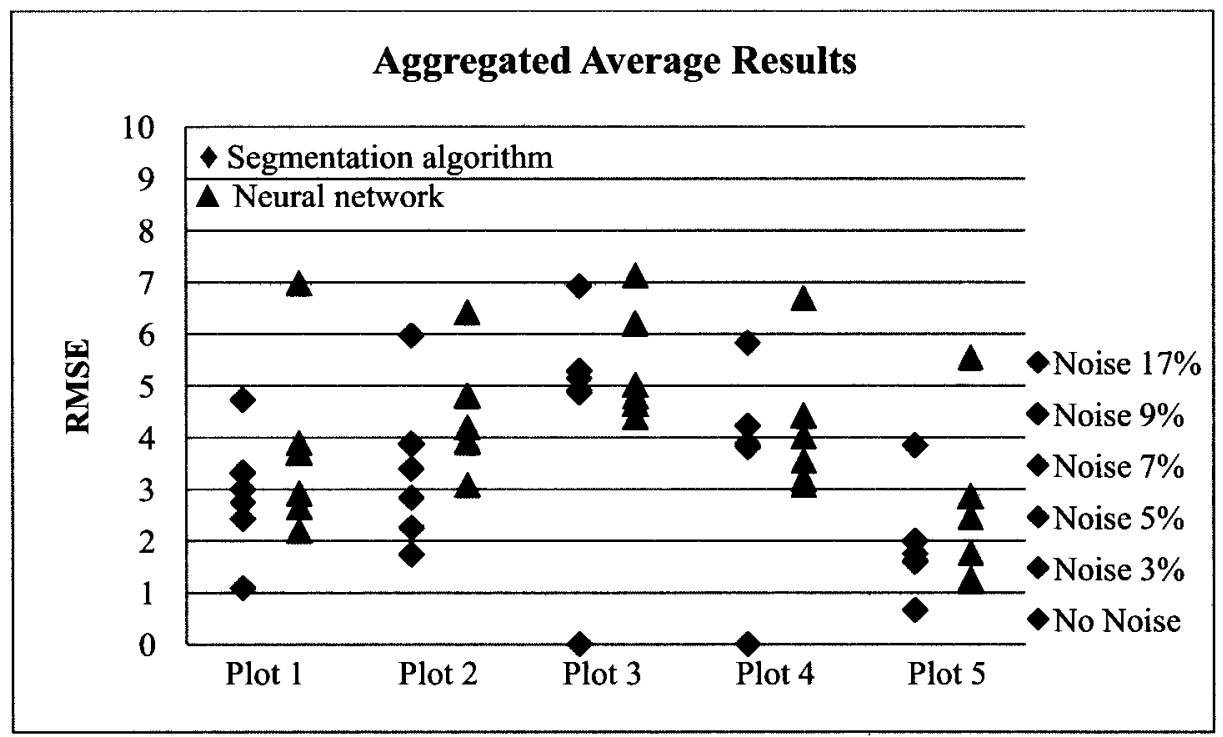

Figure 5.44: RMSE for all noise levels and plots for both data mining techniques

Distinguishing between the trend types, the segmentation algorithm can handle noise added to slow trends much better than the neural network (Figure 5.11 to Figure 5.15 and Figure 5.27 to Figure 5.31). The segmentation algorithm also produces Steady segments more readily than the neural network because the neural network most often outputs Increasing/ Decreasing segments. Outputting Steady segments aids the clinicians by providing them information when a client's health has remained constant.

However, if the 'No Noise' results (which have been optimized for each plot and each data mining technique), are considered equal between the two techniques, the neural 
network demonstrates its ability to handle the addition of noise better than the segmentation algorithm. This can be seen by calculating the $\%$ difference between the 'No Noise' RMSE value and the RMSE value for each level of noise. For all plots at all levels of noise, the neural network is able to handle the addition or noise better than the segmentation algorithm (Figure 5.45). In 19/25 of the results, the neural network outperforms the segmentation algorithm by a $100 \%$ difference. Results of Plot 3 and 4 for the segmentation algorithm were too large to be included in Figure 5.45.

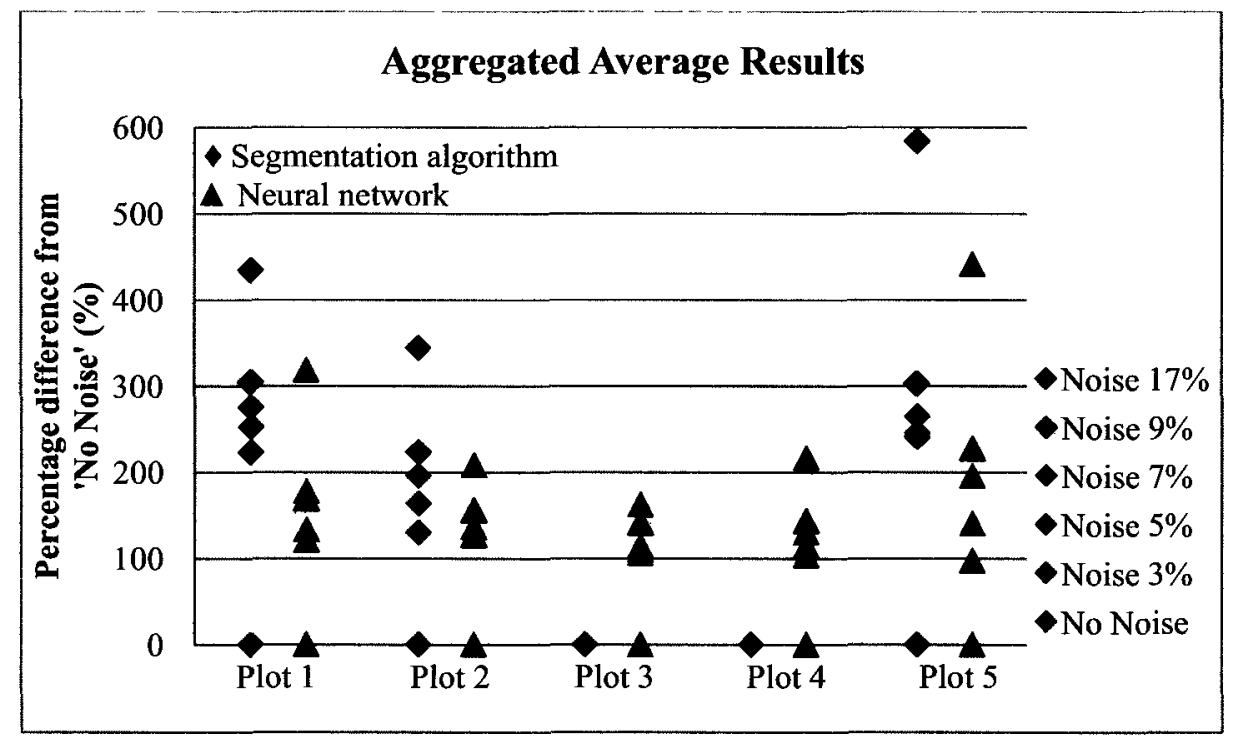

Figure 5.45: \% difference for all noise levels and plots for both data mining techniques

An important aspect to discuss is whether or not the data mining techniques are able to detect the underlying trends with the additional noise levels accurately. One option is to analyze the results qualitatively to note which features the techniques are no longer able to represent well. Another option is to analyze them in a quantitative manner.

The following describes one way of analyzing the success of the mining techniques quantitatively. The 'No Noise' RMSE results of all plots and for both 
techniques were normalized and represented as true "No Noise" values of zero RMSE. Each level of noise was also normalized.

In the case of the example plots used in this dissertation, the noise levels represent the magnitudes indicated in Table 5.7.

Table 5.7: Noise levels (\%) and their corresponding magnitudes

\begin{tabular}{|c|c|}
\hline Noise Level (\%) & Magnitude \\
\hline 3 & 0.9 \\
\hline 5 & 1.5 \\
\hline 7 & 2.1 \\
\hline 9 & 2.7 \\
\hline 17 & 5.0 \\
\hline
\end{tabular}

Analyzing Figure 5.46, it can be noted that the segmentation algorithm cannot handle noise well for Plot 3 and 4. The results for these two plots were not normalized because "No Noise" was already so close to zero. Otherwise, both techniques do well for detecting the underlying trends without having RMSE values higher than the corresponding level of noise. 


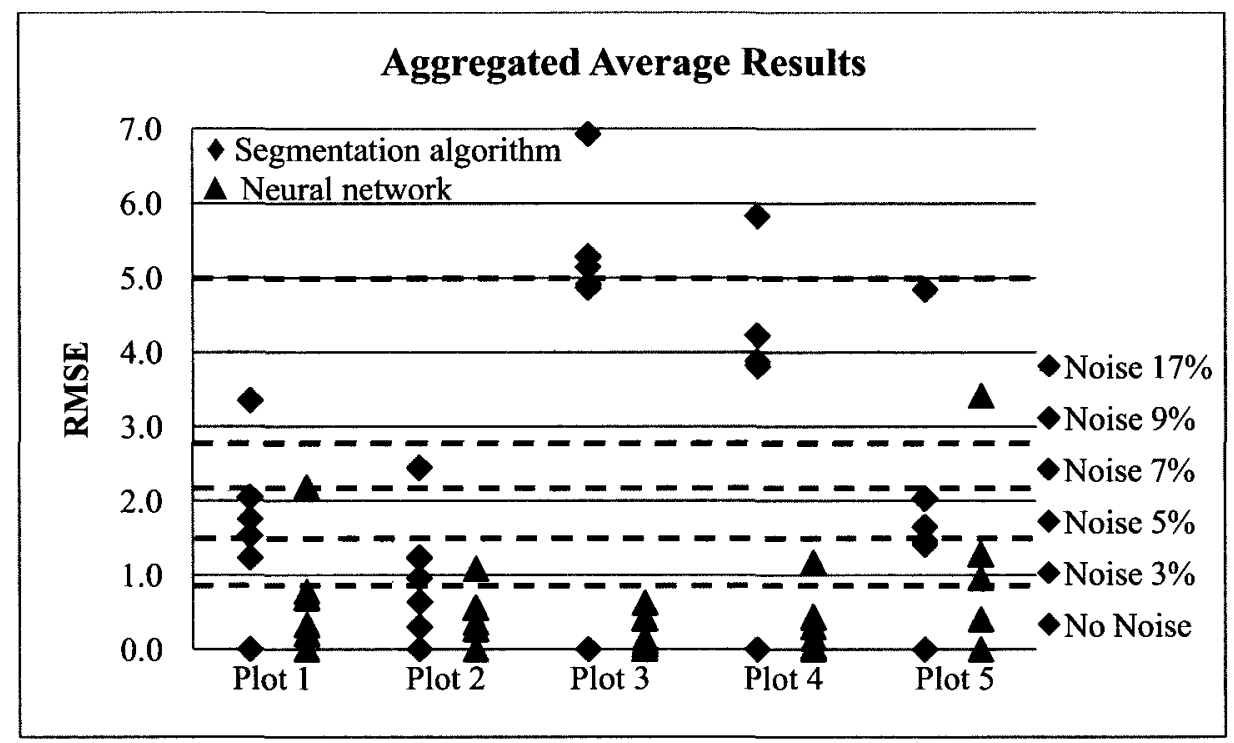

Figure 5.46: Normalized RMSE for all noise levels and plots for both data mining techniques

Using the optimal parameter values, the RMSE values for the segmentation algorithm, a neural network with seven primitives, and a neural with three primitives can be compared (Figure 5.47). The results indicate that using a neural network with seven primitives results in similar or lower RMSE values than a neural network with three primitives in the conditions tested. This indicates an area of improvement using the neural network. 


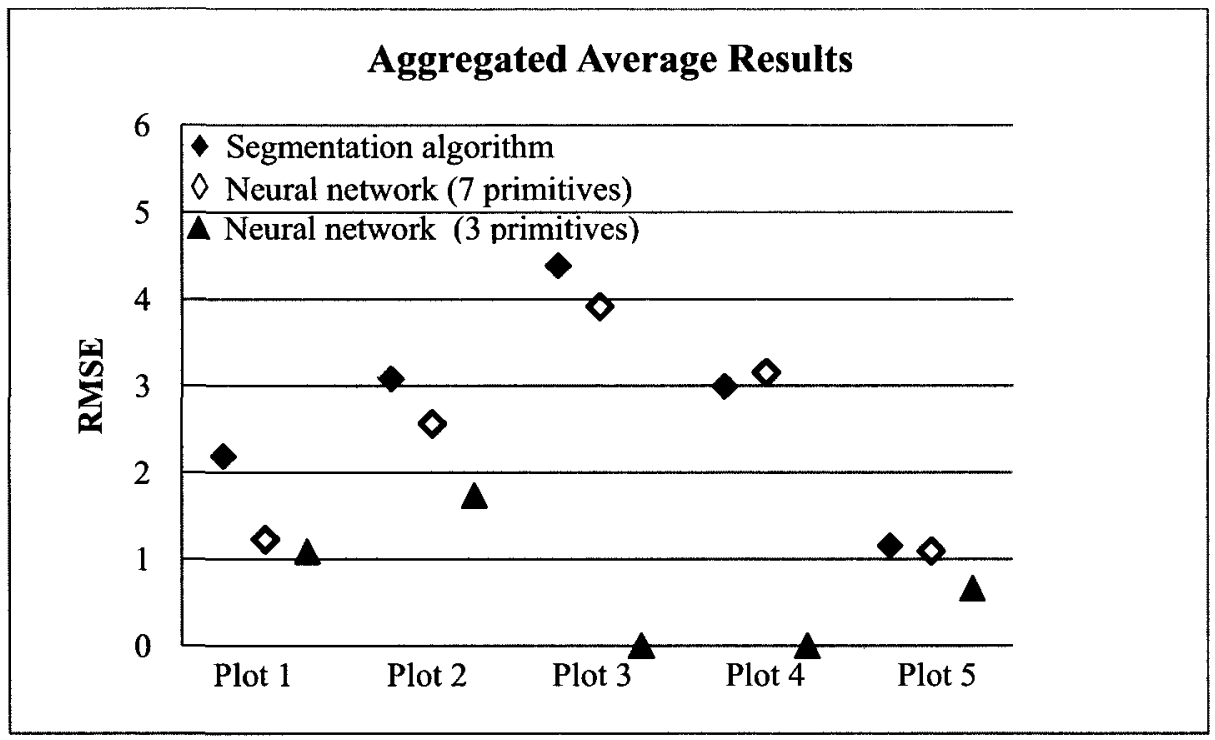

Figure 5.47: Aggregated average results for the segmentation algorithm, feed-forward neural network using 7 and 3 primitives 


\section{Chapter 6}

\section{Conclusions}

This chapter contains a summary of results and proposals for further research.

\subsection{Summary of Results}

Remote patient monitoring technology enables home care providers to view continuous data regarding their home care clients. This technology can aid clinicians in decision making and can alert them of clients' conditions before they become more acute. This research focused on two important components of remote patient monitoring: the graphical user interface of the decision support system and mining and analyzing the health data of the home care client.

\subsubsection{Graphical User Interface}

The development of the user-centered decision support system in this dissertation involved interdisciplinary groups. Users who represented the cross-section of home health care providers were solicited for information throughout this research. This allowed for a holistic view of the entire home care process and therefore, increases the likelihood of user acceptance: that the system will be accepted and used in clinical practice. Designing information and communication flow which will succeed in different work situations, as in the case of home care, with intuitive visualization and interaction 
techniques, helps lead to systems which better support clinical work processes for a variety of care providers [145].

As demonstrated in acute care settings [146] it is very important to detect trends of health parameters to more accurately determine the state of a patient. This statement was confirmed through research conducted for this thesis and was supported through focus groups of home care clinicians. The home care clinicians involved in this research indicated that important features for the graphical user interface (GUI) included displaying historical trends and some form of alert to indicate if the client was deviating from their normal state. They also desired a wide variety of client data because the need for specific data is context dependent. Graphical representation of data was preferred to numeric.

The home care clinicians also indicated three main client groups that would benefit the most from remote monitoring technologies. These groups included: clients with mobility issues, with cognitive impairments, and those who require continuous monitoring due to high-risk conditions.

Results from the usability test of the two prototype interfaces with the home care clinicians can be summarized into detailed and conceptual design issues. Improvements of the detailed design issues include: re-locating y-axis descriptions and graph legends, incorporating more colours, and permitting pop-ups as the user scrolls over data points on graphs.

Conceptual design features which were well received include: the amount of graphs and information on the home page (Version ' $A$ ' had graphs for each of the 18 parameters, Version ' $\mathrm{B}$ ' aggregated the parameters into 4 polar displays) and within any 
of the major tabs (Version ' $A$ ' and 'B' had 6 at the second level) provided the right amount of information, the use of red as an indicator for an alert, targets on graphs, and the ability to explode graphs, view weekly and daily data, and view historical trends. Improvements of the conceptual design issues include: making the sparklines clearer and more logical, providing definitions for graphs, tabs, etc. as the user scrolls over them, and a warning trend colour.

Soliciting feedback from home care clinicians regarding their requirements for exploring client data sets is crucial [147]. The two key areas of "understanding and trust" [148], are the driving forces behind visualizing data mining models. If home care clinicians can understand what has been discovered in the context of a health issue, how the analytics and data mining has been applied, they will trust the data and the underlying model and proceed to use it in their decision making. Visualizing a model, such as demonstrated in Chapter 5, allows "a user to discuss and explain the logic behind the model to others and in this way, the overall trust in the model increases and subsequent actions taken as a result are justifiable" [148]. In summary, home care clinicians will be able to understand and trust the knowledge from a data mining technique by the proper visualization of the results [110].

\subsubsection{Data Mining}

In order to support the needs of the home care clinicians identified from the focus groups, techniques for data mining were examined because they allow extraction of trends from data providing an important tool for clinical decision making. Raw data from a smart home sensor alone may not be sufficient to allow a home care clinician to differentiate between normal and abnormal situations. These techniques can help present 
the data in a manner that reflects the important underlying trends and events of health data of a home care client.

The first technique considered was a segmentation algorithm which splits a client's data into linear segments and the second technique was a feed-forward neural network.

One of the benefits of the segmentation algorithm technique is that it does not require any pre-filtering of the data because the algorithm performs signal filtering which in turn, does not distort steep changes like a traditional low-pass filter. The algorithm can classify discontinuous or continuous shapes and outputs simple, semi-qualitative episodes and a straightforward graph consisting of \{Steady, Increasing and Decreasing\} segments. A disadvantage of the technique is that it needs some a priori knowledge of the parameters monitored in order to establish threshold values.

A benefit of employing a neural network as a machine learning algorithm includes its ability to handle large size data samples and integrate background knowledge into the analysis. A disadvantage of the technique is that is needs all the data to be included in the window (i.e. the input units) before it can notify the user of a trend. Decreasing the number of input units so that a trend is outputted sooner, causes the results to be too noisy and long, slow trends not to be detected. On the other hand, the segmentation algorithm outputs an up-to-date trend by analyzing each data point as it receives them.

Given the data sets in this research, the segmentation algorithm was able to adequately detect trends that consisted of the seven primitives outlined in sub-section 5.2.2. It did however, have a difficult time with some of the data trend types with the addition of noise. When optimized for lower frequency trends, the algorithm was able to 
more accurately detect data trends than when optimized at higher frequencies. The segmentation algorithm was more accurate than the neural network in detecting long or slow trends. This could indicate its ability to be used as the mining technique for case managers whom view the health of a home care client over a few months and want aggregated results.

Presented with the same data sets, the neural network was not as successful at accurately detecting trends as the segmentation algorithm. This was determined by calculating the root-mean squared error (RMSE) for each trend type (Plot $1-5$ ). However, the neural network demonstrated good capability of handling additional noise to the different trend types by exhibiting a smaller range in RMSE values for all trend types than the segmentation algorithm. Therefore, if the data is very noisy, the neural network should be employed. For more accuracy and simpler, semi-qualitative output, the segmentation algorithm should be used. For example, the neural network may be appropriate for mining bed exit data from a bed pressure sensor mat of a client who has a large variance in bed exit behaviour. The segmentation algorithm may be best for clients who manage, or have help managing, their condition better and have less variance in their data but need to be monitored for slow and long trends.

The neural network showed similar characteristics to that of the segmentation algorithm for the process of establishing its parameters. The network had greater accuracy when trained with slower frequencies trends than when trained with high frequency trends.

A neural network which can detect seven primitives instead of three was also evaluated. In the conditions tested, the results indicated that using a network with seven 
primitives resulted in similar or lower RMSE values than a neural network with three primitives. This indicates an area of improvement if the clinicians desire more shapes (i.e. more than the primitives of $\{$ Steady, Increasing and Decreasing $\}$ ) and accuracy describing a client's health status in their results.

This dissertation has compared and evaluated two methodologies in terms of the set of desirable characteristics outlined by the home care clinicians. This research reveals the relative strengths and weaknesses of the two mining techniques and concludes that no single method has all the desirable features one would like a diagnostic system to possess. Some of the results indicate features which complement each other and if integrated, is one way to develop a hybrid system that may overcome the weaknesses of the individual techniques.

Incorporating remote monitoring sensors, like a bed pressure sensor mat, into a home care client's residence has the advantage of continuously monitoring their health. Successfully mining the data to detect abnormal situations is an improvement to the current system in which paper charts provide only sporadic snapshots that may not accurately represent the state and health of the client.

\subsection{Recommendations for Further Research}

This section presents recommendations for further research for both the GUI and data mining techniques. These recommendations include additional experimental data to acquire and new GUI features and data processing methods to investigate.

\subsubsection{Graphical User Interface}

Further iterations of the GUI design are needed followed by additional usability tests from both a broader population and a more targeted population. 
While data was acquired from 8 home care clinicians which included: an occupational therapist, three nurses and four case managers, testing an improved interface with more participants from these user groups would ensure that the design has a general application.

Data acquired from the fourth primary user group, physiotherapists, may prove to include data that vary from that of the other three groups. This primary user group was not involved in the usability test in this research because participants could not be recruited in time. It is recommended that usability tests be conducted to acquire data specifically from this group.

Data acquired from home care clients, the secondary users, may also prove to include data that vary from that of the primary users. The involvement of this group and their relatives into the system development process challenges the "organization of the health care system and introduces a new patient role - the one of an active participant and supervisor of the health care process instead of a passive recipient" [145]. It is recommended that usability tests be conducted with this group as well.

Additional research is needed to determine other alarm display possibilities and when an alarm needs to be sent to a clinician. For example, if a bed pressure sensor mat is employed and a home care client's number of bed exits drastically increases, what is the critical data point, or what are the critical parameters as to how and when an alarm should notify a clinician that this was an abnormal event? From the data mining techniques, quantitative information can be used to start or stop an alarm and qualitative information can be used to recognize specific alarm situations [131]. In summary, further 
research is needed to identify criteria and parameters for alerting clinicians of abnormal events.

The prototype interfaces developed in this research contained a great deal of information regarding the health of a fictitious client and this was voiced as a concern by the clinicians. When an application contains so much information for a variety of user groups, it can be impossible to represent the depth and breadth of the application's content in a single home page. Additionally, users have specific interests or goals in mind when consulting the interface. In such cases, it is often advantageous to use the home page to split the user groups immediately into "interest groups and to offer them specific, more relevant information in menu pages deeper within" [109] the application. It is recommended that the interface be customized to each of the four primary user groups to satisfy this requirement.

Additional usability tests on higher-fidelity prototypes (i.e. prototypes with more detail and functionality) would allow for more sophisticated parameters to be captured by the observers and researchers. Observational data that can be collected includes: recording mouse movements, observing the path used to complete each task, and recording errors and other key moments such as when participants made the same types of errors repeatedly across tasks. Additionally, the individual tendencies and decisionmaking processes can be observed if participants are allowed to complete each tasks without interruption [42].

These additional usability tests can also be used to solicit feedback on the data mining techniques. For example, do the clinicians understand and like the output of semiqualitative results? Do the qualitative primitives help data interpretation and decision- 
making? Do the clinicians prefer three primitives or seven on the data visualization graphs? Would they like the graphs to include both the model and the original data? This concept, the trade-off between accuracy versus understanability, should be greatly discussed during these tests.

\subsubsection{Data Mining}

Further testing of the data mining techniques presented in this research using more data types is needed to verify the findings and discover other strengths and limitations.

Health data acquired from home care clients along with corresponding clinical observations may prove helpful in determining the usefulness of the techniques. It is recommended that tests be conducted to acquire real-life results so as to improve the mining techniques and improve on the suggestions of establishing the parameter values of the techniques (i.e. employing the techniques in other scenarios).

It is also wise to explore other algorithms and techniques whenever possible [56]. For example, these could be Kalman filters, and autoregressive and phase-space models.

It is also recommended to design and develop a system which would be able to choose the best algorithm to fit a certain data type automatically. Additional research is needed to verify the accuracy of this method with a variety of data types.

Further testing of the composition of the neural network and the segmentation algorithm is recommended. In this research, the majority the parameters of both data mining techniques were augmented in sub-sections 5.3 .3 and 5.4 .4 when establishing parameter values. Nevertheless, it is an iterative process that is dependent on time constraints and the variety and type of data. For example, a parameter to augment in the segmentation algorithm is the number of samples that are needed between $t h_{1}$ and $t h_{2}$ 
before a new linear segment is calculated. In literature [65] and in this research the number of samples was three. For the neural network, other training functions could be explored and a network with two hidden layers could be tested. 


\section{List of References}

[1] W. Sun, H. Nguyen, D.M. Doran, R. Goubran, S. Doherty, F. Knoefel, A. Tosine, V. Joshi, and E. Sadowski, "Integration of Bio-mobility Information with at Pointof-Care Decision Support System for Safer Client Care," presented at the Canadian Nursing Informatics Association, Mississauga, ON, November 22-25, 2009.

[2] W. Sun, H. Nguyen, D.M. Doran, R. Goubran, S. Doherty, F. Knoefel, A. Tosine, V. Joshi, and E. Sadowski, "Integration of Bio-mobility Information with at Pointof-Care Decision Support System for Safer Client Care," presented at the Sigma Theta Tau 23rd Annual Research Conference: 'Promoting Excellence in Nursing Practice Through Research'. London, ON, April 30, 2010.

[3] H. Nguyen, D.M. Doran, R. Goubran, S. Doherty, F. Knoefel, A. Tosine, H. Nguyen, V. Joshi, W. Sun, and E. Sadowski, "Integration of Bio-mobility Information with at Point-of-Care Decision Support System for Safer Client Care," presented at the 4th National Community Health Nurses Conference, Toronto, ON, June 16-18, 2010.

[4] A. Tosine, H. Nguyen, V. Joshi, D. Doran, A. Parush, R. Goubran, S. Doherty and E. Sadowski, "User Research of a Proposed Home Care Clinician's Non-intrusive Remote Monitoring System's Graphical User Interface," poster presentation at the conference of e-Health 2010, Vancouver, BC, May 30 - June 3, 2010.

[5] S. Doherty, R. Goubran, D. Doran, A. Tosine, and H. Nguyen, "An automated Remote Monitoring System of Homecare Client's Nightly and Daily Activity Patterns: System Design and Outputs," poster presentation at the conference of $e$ Health 2010, Vancouver, BC, May 30 - June 3, 2010.

[6] D. Doran, R. Goubran, S. Doherty, F. Knoefel, A. Tosine, H. Nguyen, V. Joshi, W. Sun and E. Sadowski, "Technology Integration Into Home Care Nursing: Meeting the Challenges of Chronic Disease Management," poster presentation at the conference of e-Health 2010, Vancouver, BC, May 30 - June 3, 2010.

[7] L. Martel and E.C. Malenfant, 2006 Census: Portrait of the Canadian Population in 2006, by Age and Sex. Ottawa, Canada: Ministry of Industry, 2006. 
[8] "Private Home Care - a vital component of the health care continuum in Ontario," Ontario Home Care Association, September 2010, [Online]. Available: http://www.homecareontario.ca/public/docs/publications/position\%20papers/2010/p rivate-home-care-a-vital-component-of-health-care-contimuum-in-ontario.pdf

[9] K. Wilkins and E. Park, "Home care in Canada," Health Reports, Summer, Vol. 10, No. $1,1998$.

[10] Lang, N. Edwards, and A. Fleiszer, "Safety in Home Care: A broadened perspective of patient safety," Int. J. Quality in Health Care, vol. 20, no. 2, pp. 130-135, 2008.

[11] G. Demiris et al., "Nurse Participation in the Design of User Interfaces for a Smart Home System," in Proc. Int. Conf. Smart Homes and Health Telematics, Belfast, N Ireland, 2006, pp 66-73.

[12] E. Madigan and R. Fortinsky, "Alternative measures of resource consumption in home care episodes", Public Health Nursing, vol. 16, no. 3, pp. 198, 1999.

[13] M. H. Jones, A. Arcelus, R. Goubran, and F. Knoefel, "A Pressure Sensitive Home Environment," IEEE Int. Workshop on HAVE, pp. 10-14, 2006.

[14] P. Tang and T. Venables, "Smart' homes and telecare for independent living," $J$. Telemedicine and Telecare, vol. 6, no.1, p. 8, 2000.

[15] K.D. Blanchet, "Remote Patient Monitoring," Telemedicine J. and e-Health, vol. 14 , no. 2, pp. 127-130, 2008.

[16] G. Demiris et al., "End-User Involvement in Interface Design of Smart Home Applications: The Nursing Perspective," Int. Conf. Ageing, Disability and Independence, Feb 1-5, 2006, St. Petersburg, USA.

[17] C.N. Scanaill et al. "Evaluation of an Accelerometer-Based Mobility Telemonitoring Device in a Smart Home Environment," Proc. Smart Homes and Beyond, IOS Press, C. Nugent and J. C. Augusto, Eds., 2006.

[18] G. Virone et al. "An Assisted Living Oriented Information System Based on a Residential Wireless Sensor Network," Proc. $1^{\text {st }}$ Distributed Diagnosis and Home Healthcare Conf. Arlington, VA, 2006, pp. 95-100.

[19] F.G. Miskelly, "Assistive technology in elderly care," Age and Ageing, vol. 30, no. 6, pp. 455-458, 2001.

[20] A. Arcelus, M. Howell-Jones, R. Goubran, and F. Knoefel, "Integration of Smart Home Technologies in a Health Monitoring System for the Elderly," in Proc. Int. Conf. Advanced Inform. Networking and Applicat. Workshops, ON, Canada, May 2007, pp $820-825$. 
[21] J.M. Choi et al., "A System for Ubiquitous Health Monitoring in the Bedroom via a Bluetooth Network and Wireless LAN," EMBC2004 Conf. Proc. $26^{\text {th }}$ Annual Int. Conf. IEEE, vol. 2, pp. 3362-3365, 2004.

[22] A.M. Adami et al., "Detection and classification of movements in bed using load cells," IEEE 27 $7^{\text {th }}$ Annual Int. Conf. Eng. Medicine and Biology Society, pp. 589$592,2005$.

[23] H.F.M. Van der Loos, N. Ullrich, and H. Kobayashi, "Development of Sensate and Robotic Bed Technologies for Vital Signs Monitoring and Sleep Quality Improvement," Autonomous Robots, vol. 15, no. 1, pp. 67-69, 2003.

[24] Y. Nishida et al., "Monitoring patient respiration and posture using human symbiosis system," in Proc. 1997 IEEE Int. Conf. Intelligent Robots and Systems, vol. 2, pp. 632-639, 1997.

[25] C. Cheng and C. Young, "Development of a Portable Device for Telemonitoring of Physical Activities During Sleep," Telemedicine and e-Health, vol. 14, no. 10, pp. 1044-1056, 2008.

[26] J. Alihanka and K. Vaahtoranta, "A static charge sensitive bed - A new method for recording body movements during sleep, "Electroencephalogr Clin Neurophysiol, vol. 46, no. 6, pp. 731-734, 1979.

[27] E. Rauhala, M. Erkinjuntti and O. Polo, "Detection of periodic leg movements with a static-charge-sensitive bed," J. Sleep Res, vol. 5, no. 4, pp. 246-250, 1996.

[28] J. Kaartinen, I. Kuhlman and P. Peura, "Long-term monitoring of movements in bed and their relation to subjective sleep quality," Sleep Hypnosis, vol. 5, no. 3, pp. 145$153,2003$.

[29] M.J. Rantz et al., "Developing a Comprehensive Electronic Health Record to Enhance Nursing Care Coordination, Use of Technology, and Research," $J$ of Gerontological Nursing, vol. 36, no. 1, pp. 13-17, 2010.

[30] T. Tamura, A. Nishigaichi, and T. Nomura, "Monitoring of body movement during sleep in bed," Proc. Annu. Int. Conf. IEEE Eng. Medicine and Biology Society, vol. 14, pp. 1483-1484, 1992.

[31] W.B. Spillman et al., "A 'smart' bed for non-intrusive monitoring of patient physiological factors,” Meas. Sci. Technol., vol. 15, pp. 1614-1620, 2004.

[32] H. Kimura et al., "Development of an unobtrusive vital signs detection system using conductive fiber sensors," Proc. 2004 IEEE/RSJ Int. Conf. Intelligent Robots and Systems, vol. 1, pp. 307-312, 2004. 
[33] “About HTES," Human Factors and Ergonomics Society, August 2000, [Online]. Available: http://www.hfes.org/web/AboutHFES/about.html [Accessed: Sep 28, 2010].

[34] C. D. Wickens, J.D. Lee, Y. Liu, and S.E.G. Becker, An Introduction to Human Factors Engineering, $2^{\text {nd }}$ Ed. Upper Saddle River, NJ: Pearson Prentice Hall, 2004, pp. 2 - 35.

[35] T. H. Payne, "Computer Decision Support Systems," Chest, vol. 118, no. 2, pp. 47S-52S, 2000.

[36] P.C. Coyte and P. McKeever, "Home care in Canada: passing the buck," Canadian J. Nursing Research, vol. 33, no. 2, pp. 11-25, 2001.

[37] D.A. Norman and S.W. Draper, Eds., User Centered System Design: New Perspectives on Human-Computer Interaction. Hillsdale, NJ: Lawrence Erlbaum Associates, 1986, p. 61.

[38] J. Nielsen, Usability engineering. New York, NY: Academic Press, 1993.

[39] R. Schoeffel, “The concept of product usability,” ISO Bulletin, vol. 6, pp. 6-7, 2003.

[40] I. Scandurra, M. Hägglund, S. Koch and M. Lind, "Usability Laboratory Test of a Novel Mobile Homecare Application with Experienced Home Help Service Staff," Open Medical Informatics, vol. 2, pp. 117-128, 2008.

[41] K.A. Smith, "Neural Networks for Prediction and Classification", Encyclopaedia of Data Warehousing and Mining, J. Wang, Ed., vol. II, I-Z, Hershey, PA: Idea Groups Reference, 2006.

[42] S.J. Fonda et al., "Usability Test of an Internet-Based Informatics Tool for Diabetes Care Providers: The Comprehensive Diabetes Management Program," Diabetes Technology \& Therapeutics, vol. 10, no. 1, pp. 16-24, 2008.

[43] G.R. Casper et al., "Designing a Technology Enhanced Practice for Home Nursing Care of patients with Congestive Heart Failure," Proc. AMIA 2005 Symposium, pp. 116-120, 2005.

[44] J.M. Jung et al., "XML based on-line Clinical Decision Support System for Home Healthcare," IFMBE Proc, vol. 14, no. 6, pp. 4135-4138, 2007.

[45] B. Biswas, M. Jayachandran, L. Shue, W. Xiao, and P. Yap, "An Extensible System for Sleep Activity Pattern Monitoring," $3^{\text {rd }}$ Int. Conf. Intelligent Sensors, Sensor Networks and Inform., pp. 561-565, 2007.

[46] C.D. Krivda, Data-Mining Dynamite. Byte, Oct 95: 97-102, 1995. 
[47] S. Theodoridis and K. Koutroumbas, Pattern Recognition. San Diego, CA: Academic Press, 1999.

[48] S.I. Weiss and C. Kulikowski, Computer Systems That Learn: Classification and Prediction Methods from Statistics, Neural Networks, Machine Learning, and Expert Systems. San Francisco, CA: Morgan Kaufmann, 1991.

[49] D.J. Hand, Discrimination and Classification. Chichester, U.K.: Wiley, 1981.

[50] U. Fayyad, G. Piatetsky-Shapiro and P. Smyth, "From Data Mining to Knowledge Discovery in Databases," AI Magazine, vol. 17, pp. 37-54, 1996.

[51] A.K. Jain and R.C. Dubes, Algorithms for Clustering Data. Englewood Cliffs, NJ: Prentice-Hall, 1988.

[52] D.M. Titterington, A.F.M. Smith, and U.E. Makov, Statistical Analysis of FiniteMixture Distributions. Chichester, UK: Wiley, 1985.

[53] B. Thuraisingham et al., "Data Mining Techniques," Design and Implementation of Data Mining Tools. Boca Raton: Auerbach Publications, 2009.

[54] J. Liu, J.C. Wyatt and D.G. Altman, "Decision tools in health care: focus on the problem, not the solution", BMC Med. Informatics and Decision Making, vol. 6, no. 4, pp. 2006.

[55] E.A. Mendonca, “Clinical Decision Support Systems: Perspectives in Dentistry," J. Dental Education, vol. 68, no. 6, pp. 589-597, 2004.

[56] G. Meyfroidt, F. Guiza, J. Ramon, and M. Bruynooghe, "Machine learning techniques to examine large patient databases", Best Practice \& Research Clinical Anaesthesiology, vol. 23,no. 1, pp. 127-143, 2009.

[57] W. Raghupathi, "Designing Clinical Decision Support Systems in Health Care: A Systemic Review," Int. J. Healthcare Inform. Systems and Informatics, vol. 2, no. 1, pp. 44-53, 2007.

[58] S. Charbonnier, "On line extraction of temporal episodes from ICU high-frequency data: A visual support for signal interpretation," Computer Methods and Programs in Biomedicine vol. 78, no. 2, pp. 115-132, 2005.

[59] N. Lavrac and B. Zupan, "Mining Time in Medicine," Data Mining and Knowledge Discovery Handbook, O. Maimon and L. Rokach, Eds. New York, NY: Springer, 2005.

[60] M. Imhoff et al., "Smart Alarms from medical devices in the OR and ICU," Best Practice \& Research Clinical Anaesthesiology, vol. 23, no. 1, pp. 39-50, 2009. 
[61] A.F. Siegel, "Robust regression using repeated medians," Biometrika, vol. 69, no. 1, pp. 242-244, 1982.

[62] P.L. Davies, R. Fried and U. Gather, "Robust signal extraction for on-line monitoring data," J. Statistical Planning and Inference, vol. 122, no. 1-2, pp. 65-78, 2003.

[63] R. Fried, "Robust filtering of time series with trends," J. Nonparametric Statistics, vol. 16, no. 33, pp. 313-328, 2004. [Special Edition].

[64] U. Gather, K. Schettlinger and R. Fried, "Online signal extraction by robust linear regression," Computational Statistics, vol. 21, no. 1, pp. 33-51, 2006.

[65] S. Charbonnier and S. Gentil, "On-line adaptive trend extraction of multiple physiological signals for filtering in intensive care units," Int. J. Adaptive Control and Signal Processing, vol. 24, no. 5, pp. 382-408, 2009.

[66] S. Charbonnier et al., "Segmentation algorithm for ICU continuously monitored clinical data," 15 $5^{\text {th }}$ World IFAC congress, 2002.

[67] A. Mäkivirta, E. Koski, A. Kari, and T. Sukuvaara, "The median filter as a preprocessor for a patient monitor limit alarm system in intensive care," Computer Methods and Programs in Biomedicine, vol. 34, no. 2-3, pp. 139-144, 1991.

[68] W.A. Shewhart, $c$, Princeton, NJ: D. Van Nostrand Reinhold, 1931.

[69] R.R. Kennedy, “A modified Trigg's tracking variable as an 'advisory' alarm during anaesthesia," Int. J. Clinical Monitoring and Computing, vol. 12, no. 4, 197-204, 1995.

[70] A.F.M. Smith et al., "Monitoring kidney transplant patients," Statistician, vol. 32, no. $1-2$, pp. $46-54,1983$.

[71] I.M. Trimble et al., "Detection of renal allograft rejection by computer," $B M J$, vol. 286, no. 6379, pp. 1695-1699, 1983.

[72] M. Daumer and M. Falk, "Online change point detection (for state space models) using multi-process Kalman filters," Linear Algebra and its Applications, vol. 284, no. 1 , pp. 125-135, 1998.

[73] K. Gordon and A.F.M. Smith, "Modeling and monitoring biomedical time series," J. Amer. Statistical Association, vol. 85, no. 410, pp. 328-337.

[74] P. Yang et al., "Adaptive change detection in heart rate trend monitoring in anaesthetized children," IEEE Trans. Bio-medical Eng., vol. 53, no. 11, pp. 2211$2219,2006$. 
[75] C.K.I. Williams et al., "Factorial switching Kalman filters for condition monitoring in neonatal intensive care," Advances in Neural Information Processing Systems, vol. 18, Cambridge, MA: MIT Press, 2006.

[76] M. Imhoff et al., "Time series analysis in intensive care medicine," Applied Cardiopulmonary Pathophysiology, vol. 6, pp. 263-281, 1997.

[77] M. Imhoff et al., "Statistical pattern detection in univariate time series of intensive care on-line monitoring data," Intensive Care Medicine, vol. 24, no. 12, pp. 1305 $1314,1998$.

[78] M. Imhoff et al., "Pattern detection in intensive care monitoring time series with autoregressive models: influence of the model order," Biometrical J., vol. 44, no. 6, pp. 746-761, 2002.

[79] U. Gather, M. Bauer, and R. Fried, "The identification of multiple outliers in online monitoring data," Estadistica, vol. 54, pp. 289-338, 2002.

[80] M. Imhoff and U. Gather, "Time series analysis for the alarm detection in intensive care monitoring systems," Biomedizinische Technik. Biomedical Eng., vol. 49(Suppl. 1), pp. 336-336, 2004.

[81] U. Gather, R. Fried, and M. Imhoff, "Online classification of states in intensive care," in W. Gaul, O. Opitz and M Schader, eds., Data analysis, Berlin Heidelberg: Springer, 2000, pp. 413-428.

[82] D.R. Westenskow et al., "Intelligent alarms reduce anaesthesiologist's response time to critical faults," Anesthesiology, vol. 77, no. 6, pp. 1074-1079, 1992.

[83] T. Sukuvaara et al., "A knowledge-based alarm system for monitoring cardiac operated patients-technical construction and evaluation," Int. J. Clinical Monitoring and Compturing, vol. 10, no. 2, pp. 117-126, 1993.

[84] M. Chan, P. Ringeard and E. Campo, "Smart house automation system for the elderly and the disabled", IEEE Int. Conf. Syst. Man Cybern., vol. 2, pp. 1586-1589, 1995.

[85] M. Chan, E. Campo and D. Esteve, "Assessment of elderly mobility using a remote multisensory monitoring system", Stud. Health Technol. Inform, vol. 90, pp. 72-77, 2002.

[86] M.R. Maurya, R. Rengaswamy and V. Venkatasubramanian, "Fault diagnosis using dynamic trend analysis: A review and recent developments", Eng. Applicat. Artificial Intelligence, vol. 20, no. 2, pp. 133-146, 2007.

[87] R. Rengaswamy and V. Venkatasubramanian, "A Syntactic Pattern-recognition Approach for Process Monitoring and Fault Diagnosis", Eng. Applicat. of Artificial Intelligence, vol. 8, no. 1, pp. 35-51, 1995. 
[88] C. Ulbricht, G. Dorffner and A. Lee, "Neural networks for recognizing patterns in cardiotocograms," Artificial Intelligence, vol. 12, no. 3, pp. 271-284. 1998.

[89] R.M. Farrel, J.A. Orr, K. Kuck and D.R. Westenskow, "Differential features for neural network based anesethesia alarm system," Biomedical Sci Instrumentation, vol. 28, pp. 99-104, 1992.

[90] J.A. Orr and D.R. Westenskow, "A breathing circuit alarm system based on neural network," J. Clinical Monitoring, vol. 10, no. 2, pp. 101-109, 1994.

[91] C.L. Tsien, "TrendFinder: Automated detection of alarmable trends," MIT Ph.D. dissertation, 2000.

[92] K. Becker et al., "Design and validation of an intelligent patient monitoring and alarm system based on a fuzzy logic process model," Artificial Intelligence in Medicine, vol. 11, no. 1, pp. 33-53, 1997.

[93] A. Lowe, R.W. Jones, and M.J. Harrison, "A graphical representation of decision support information in an intelligent anaesthesia monitor," Artificial Intelligence in Medicine, vol. 22, pp. $173-191,2001$.

[94] M. Wolf et al., "Improved monitoring of preterm infants by Fuzzy Logic", Technology and Health Care, vol. 4, no. 2, pp. 193-201, 1996.

[95] C. Oberli et al., "An expert system for monitor alarm integration", J. Clinical Monitoring and Computing, vol. 15, no. 1, pp. 29-35, 1999.

[96] W. Zong, G.B. Moody and R.G. Mark, "Reduction of false arterial blood pressure alarms using signal quality assessment and relationships between the electrocardiogram and arterial blood pressure," Medical \& Biological Eng.\& Computing, vol. 33, no. 5, pp. 111-115, 1994.

[97] P. Laursen, "Event detection on patient monitoring data using causal probabilistic networks," Methods of Inform. in Medicine, vol. 33, no. 1, pp. 111-115, 1994.

[98] S. Haykin, Neural Networks and Learning Machines, $3^{\text {rd }}$ ed. Upper Saddle River, NJ: Pearson Education, Inc., 2009.

[99] H. Demuth and M. Beale, Neural Network Toolbox. Natick, MA: The MathWorks, 1998.

[100] J. Friedman, R. Tibshirani and T. Hastie, Elements of Statistical Learning: Data Mining, Inference, and Predication, $2^{\text {nd }}$ ed. New York, NY: Springer, 2009.

[101] H. Sharp, Y. Rogers and J. Preece, Interaction Design, $2^{\text {nd }}$ ed. John Wiley \& Sons, Ltd.: Hoboken, NJ, 2007. 
[102] C. Borysowich, "User Characteristics - Human Factors Analysis," Toolbox for IT, Sep 2, 2007, [Online]. Available: http://it.toolbox.com/blogs/enterprisesolutions/user-characteristics-human-factors-analysis-18765 [Accessed: Sep 28, 2010].

[103] M.H. Jeon et al., "User Segmentation \& UI optimization through mobile phone log analysis," Proc. Int. Conf. Human-Computer Interaction with Mobile Devices and Services, Amerstand, Netherlands, 2008, pp. 495-496.

[104] A.W. Kushniruk and V.L. Patel, "Cognitive and usability engineering methods for the evaluation of clinical information systems", J. Biomed. Informatics, vol. 37, no. 1, pp. 56-76, 2004.

[105] T. Grossman, G. Fitzmaurice and R. Attar, "A Survey of Software Learnability; Metrics, Methodologies, and Guidelines," CHI 2009 Metrics, Boston, MA, 2009, pp. 649-658.

[106] "Services for individuals \& families," Saint Elizabeth Health Care, 2010, [Online]. Available: http://www.saintelizabeth.com/services-for-individuals-andfamilies [Accessed: Feb 26, 2010].

[107] Home care case management: Invitational roundtable. Available: http://www.hcsc.gc.ca/hcs-sss/pubs/home-domicile/2005-cas-mgmt-gest/index-eng.php [Accessed: Feb 26, 2010].

[108] B. Shneiderman, "The eyes have it: A task by data type taxonomy for information visualizations," Proc. 1996 IEEE, Visual Languages, Boulder, CO, 1996, pp. 336343.

[109] P.J. Lynch and S. Horton, Web Style Guide. Yale University Press: London, UK, 1999.

[110] H.L. Viktor and E. Paquet, "Visualization Techniques for Data Mining", Encyclopedia of Data Warehousing and Mining, J. Wang, Ed., vol. II, I-Z, Hershey, PA: Idea Groups Reference, 2006.

[111] S. Few, "Dashboard Design for Rich and Rapid Monitoring," Visual Business Intelligence Newsletter, 2006, [Online]. Available: http://www.bonavistasystems.com/Download2/Dashboard\%20Design\%20for\%20Ri ch\%20and\%20Rapid\%20Monitoring.pdf [Accessed: Aug 7, 2009].

[112] C.M. Burns and J.R. Hadjukiewicz, "Ecological Interface Design," Ecological Interface Design, pp. 1-195, 2002 (unpublished). 
[113] P.E. Hoffman and G.G. Grinstein, "A survey of visualization for high-dimensional data mining," Information visualization in data mining and knowledge discovery, U. Fayyad, G. G. Grinstein and A. Wiese, Eds., San Francisco: Morgan Kaufmann, 2001. pp. 47-82.

[114] G.N. Peterson, "Colour", GIS Cartography: A Guide to Effective Map Design, Boca Raton, FL: CRC Press, 2009.

[115] J. Nielsen, Usability Engineering. San Francisco, CA: Morgan Kaufmann, 1993.

[116] J. Rubin, Handbook of usability testing: how to plan, design and conduct effective tests. New York: Wiley; 1994.

[117] W. Hwang and G. Salvendy, "Number of People Required for Usability Evaluation: The $10 \pm 2$ Rule," Communication of the $A C M$, vol. 53, no. 5, pp. 130$133,2010$.

[118] C. G. Roehrborn, et al., "Microwave thermotherapy for benign prostatic hyperplasia with the Dornier Urowave: results of a randomized, double-blind, multicenter, sham-controlled trial", Urology, vol. 51, no. 1, pp. 19-28, 1998.

[119] K.T. McVary, Ed., Management of benign prostatic hypertrophy. Totowa, N.J: Humana Press Inc., 2004.

[120] J. D. McConnell, M.J. Barry and R.C. Bruskewitz. (1994 Feb). Benign prostatic hyperplasia: diagnosis and treatment [Online]. Available: http://www.ncbi.nlm.nih.gov/books/bv.fcgi?rid=hstat6.chapter.17571 [Accessed: Feb 12, 2010].

[121] "Energy Delivery Systems for Treatment of Benign Prostatic Hyperplasia," Ontario Health Technology Assessment Series, vol. 6, no. 17, 2006.

[122] S.J. Berry, D.S. Coffey, P.C. Walsh and L.L. Ewing, "The development of human benign prostatic hyperplasia with age," J. Urol., vol. 132, no. 3, pp. 474-479, 1984.

[123] R. W. Norman, J.C. Nickel, D. Fish, S.N. Pickett, "'Prostate-related symptoms' in Canadian men 50 years of age or older: prevalence and relationships among symptoms," Br. J. Urol., vol. 74, no. 5, pp. 542-50, 1994.

[124] D.S. Moore and G.P. McCabe, Introduction to the Practice of Statistics, $3^{\text {rd }}$ ed. New York, NY: W.H. Freeman, 1999.

[125] A.R. Webb, Statistical Pattern Recognition. West Sussex: John Wiley \& Sons, Ltd. 2002. 
[126] S. Charbonnier, G. Becq, and L. Biot, "On-line Segmentation Algorithm for Continuous Monitored Data in Intensive Care Units," IEEE Trans. Biomedical Eng., vol. 51, no. 3, pp. 484-492, 2004.

[127] D. Calvelo, M.-C. Chambrin, D. Pomorski and P. Ravaux, "Towards symbolisation using data-driven extraction of local trends for ICU monitoring," Artificial Intell. in Medicine, vol. 1, no. 2, pp. 203-223, 2000.

[128] A. Mäkivirta, E. Koski, A. Kari, and T. Sukuvaara, "Robust signal-to-symbol transformation using median filters", Proc. IFAC workshop on decision support for patient management: measurement, modelling and control, pp. 91-104, 1989.

[129] J. Kiusalaas, "Interpolation and Curve Fitting," in Numerical Methods in Engineering with Python, $2^{\text {nd }}$ ed., New York: Cambridge University Press, 2005, ch. 3 .

[130] S.R. Mutcha and R. Sharma, "Demarcation of Utterance Boundaries in Speech Signals Using Mahalanobis Distance Function as a Sample Clustering Technique," SIGAI Workshop on Emerging Research Trend in AI, Navi Mumbai, India, 2010.

[131] S. Charbonnier and S. Gentil, "A trend-based alarm system to improve patient monitoring in intensive care units," Control Eng. Practice, vol. 15, no. 9, pp. 10391050, 2007.

[132] S. Charbonnier, "On-line Extraction of Successive Temporal Sequences from ICU High-Frequency Data for Decision Support Information," Proc. $9^{\text {th }}$ Conf. Artificial Intell. in Medicine, Protaras, Cyprus, 2003, pp. 1-10.

[133] S. Charbonnier, C. Garcia-Beltan, C. Cadet, and S. Gentil, "Trends extraction and analysis for complex system monitoring and decision support," Eng. Applicat. Artificial Intell., vol. 18, no. 1, pp. 21-36, 2005.

[134] K. Yu, I. Sharp and Y.J. Guo, "Positioning Accuracy Evaluation," Ground-Based Wireless Positioning. West Sussex, England: John Wiley \& Sons Ltd, 2009, ch. 8.

[135] "IEEE Standard on Transitions, Pulses, and Related Waveforms", IEE Std. 1812003. New York, NY: IEEE, 2003.

[136] S. Miksch, W. Horn, C. Popow, and F. Paky, "Utilizing temporal data abstraction for data validation and therapy planning for artificially ventilated newborn infants", Artificial Intell. in Medicine, vol. 8, pp. 543-576, 1996.

[137] J.L. Elman, "Finding structure in time." Cognitive Sci., vol. 14, no. 2, pp. 179-211, 1990. 
[138] E. Portillo, "On the Application of Recurrent Neural Network Techniques for Detecting Instability Trends in an Industrial Process," IEEE Conf. Emerging Technologies and Factory Automation, 2007, pp. 242-248.

[139] H.K.U. Samrasinghe and S. Hashimoto, "Automated trend diagnosis using neural networks," IEEE Int. Conf. Systems, Man, and Cybernetics, vol. 2, pp. 1186-1191, 2000 .

[140] H.K.U. Samarasinghe and S. Hashimoto, "Fault detection and diagnosis system for air-conditioning units using recurrent type neural network", IEEE Conf. Syst., Man, and Cybernetics, vol. 4, pp. 2637-2642, 2000.

[141] K. Rao, P. Chand, and M. Murthy, "A neural network approach in medical decision systems," J Theoretical and Appl. Inform. Technology, vol. 3, no. 4, pp. 97-101, 2007.

[142] J.L. Guardado, J.L. Naredo, P. Moreno and C.R. Fuerte, "A comparative Study of Neural Network Efficiency in Power Transformers Diagnosis Using Dissolved Gas Analysis," IEEE Trans. Power Delivery, vol. 16, no. 4, pp. 643-647, 2001.

[143] J. Heaton, Introduction to Neural Networks with Java. Chesterfield, MO: Heaton Research, Inc., 2005.

[144] V. Venkatasubramanian, R. Vaidyanathan and Y. Yamamoto, "Process fault detection and diagnosis using neural networks - I. Steady State Process," Comput. Chem. Eng., vol. 14, 699-712, 1990.

[145] S. Koch et al., "Towards a virtual health record for mobile home care of elderly citizens," Stud. Health Technol. Inform., vol. 107, no. 2, pp. 960-963, 2004.

[146] C.A. Bellera et al., "Detecting Trends in Noisy Data Series: Application to Biomarker Series," Am. J. Epidemiol., vol. 167, no. 9, 1130-1139, 2008.

[147] M. Foster and A.G. Gee, "The data visualization environment," Information visualization in data mining and knowledge discovery, U. Fayyad, G.G. Grinstein and A. Wiese, Eds., San Francisco, CA: Morgan Kaufmann, 2001, pp. 83-94.

[148] K. Thearling et al., "Visualizing data mining models," Information visualization in data mining and knowledge discovery, U. Fayyad, G.G. Grinstein and A. Wiese, Eds., San Fransisco, CA: Morgan Kaufmann, 2001, pp. 363-371. 


\section{Appendix A: Other Medical Case Descriptions Used During the Usability Tests}

\section{Scenario 1 - Physiotherapist}

You are about to arrive at your first client's house. The client, Joe Ranger, is an 80 yearold male who has mobility issues from a broken ankle. Six weeks ago, Mr. Ranger fell and broke his ankle at home. He had surgery in the hospital and has recovered well enough to go home. Although he is ambulating independently by using a walker, he is scared of falling again and therefore minimizing his activities, including the exercises that he was advised to do. He also states that he is still finds it painful to put weight on his affected ankle and shares that this ankle is affecting his sleep. He received rehab while at the hospital but you are following up on his care in the home.

You need to review Mr. Ranger's electronic health record in your car before you enter the house so that you can assess how hiss week went and whether or not there has been any improvement with his mobility.

You access Mr. Ranger's file and need to:

- Find if he has been more sedentary this week

- Evaluate his activity patterns in the house

- Review the changes in his bed activity (e.g. his bed exit transfers) 
- Offer exercise regime to help facilitate healing and building strength in the affected ankle

\section{Scenario 1 - Occupational Therapist}

You are about to arrive at your first client's house. The client, Joe Ranger, is an 80 yearold male who has mobility issues from a broken ankle. Since his mobility is affected, you have been asked to conduct a home assessment to ensure that he can be independent in his home. Currently, he uses a walker to enhance his mobility.

You need to review Mr. Ranger's electronic health record in your car before you enter the house so that you can assess how his week went and whether or not there has been any improvement.

You access Mr. Ranger's file and need to:

- Find if he has been more sedentary this week

- Evaluate his activity patterns in the house

- Review the changes in his bed activity (e.g. bed exit transfers)

- Suggest assistive devices that can help Mr. Ranger with maintaining independence in the home

\section{Scenario 1 - Case Manager}

You need to review Joe Ranger's, an 80 year-old male who has mobility issues, electronic health record in order to determine if there as been any progress with his condition.

You access Mr. Ranger's file and need to:

- Find if he has been become more sedentary since returning home

- Evaluate his activity patterns in the house since returning home

- Review the changes in his bed activity (e.g. bed exit transfers) 
- Suggest changes to his care regime (determine if Mr. Ranger needs addition support such as meals on wheels, assistance house related chores, transportation to appointments) 


\section{Appendix B: Segmentation Algorithm - Optimal Parameter Plots}
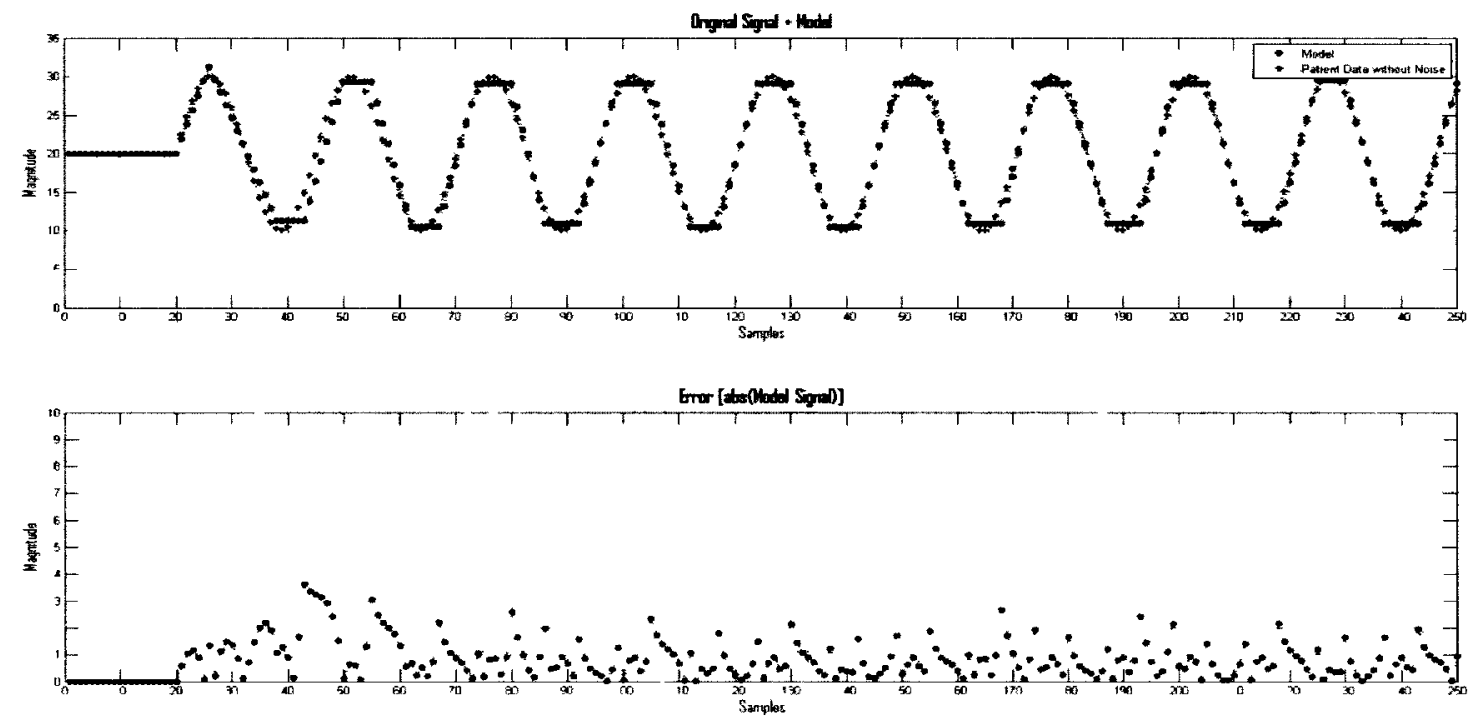

Figure B.1: Plot 1
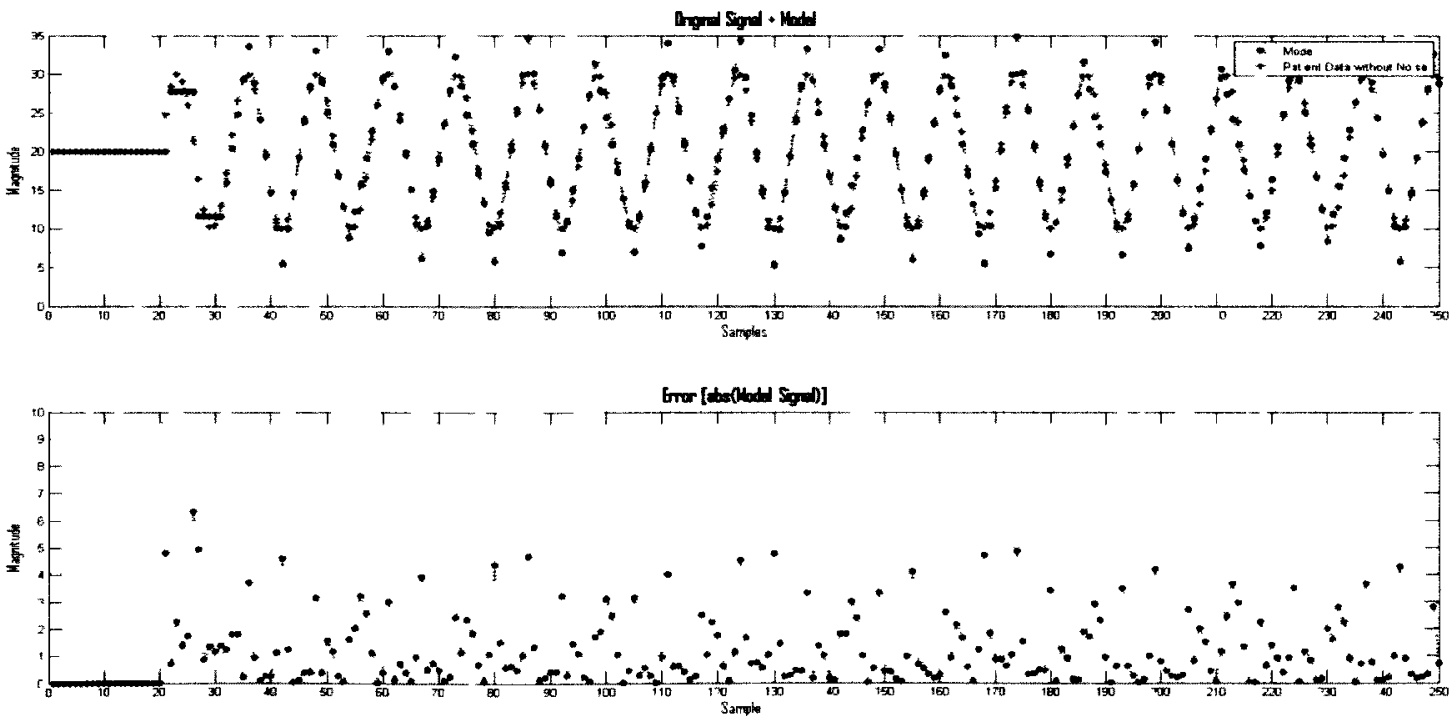

Figure B.2: Plot 2 

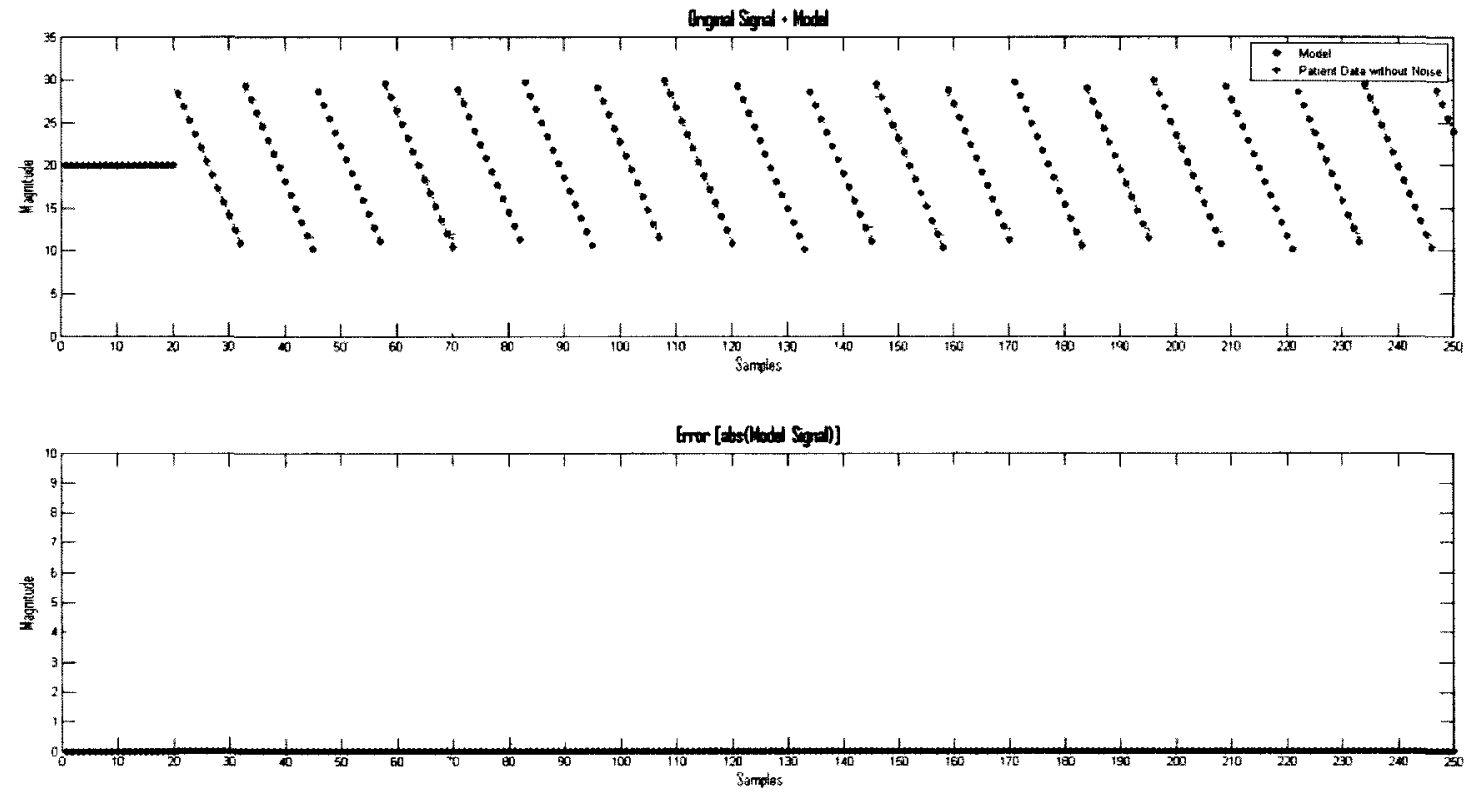

Figure B.3: Plot 3
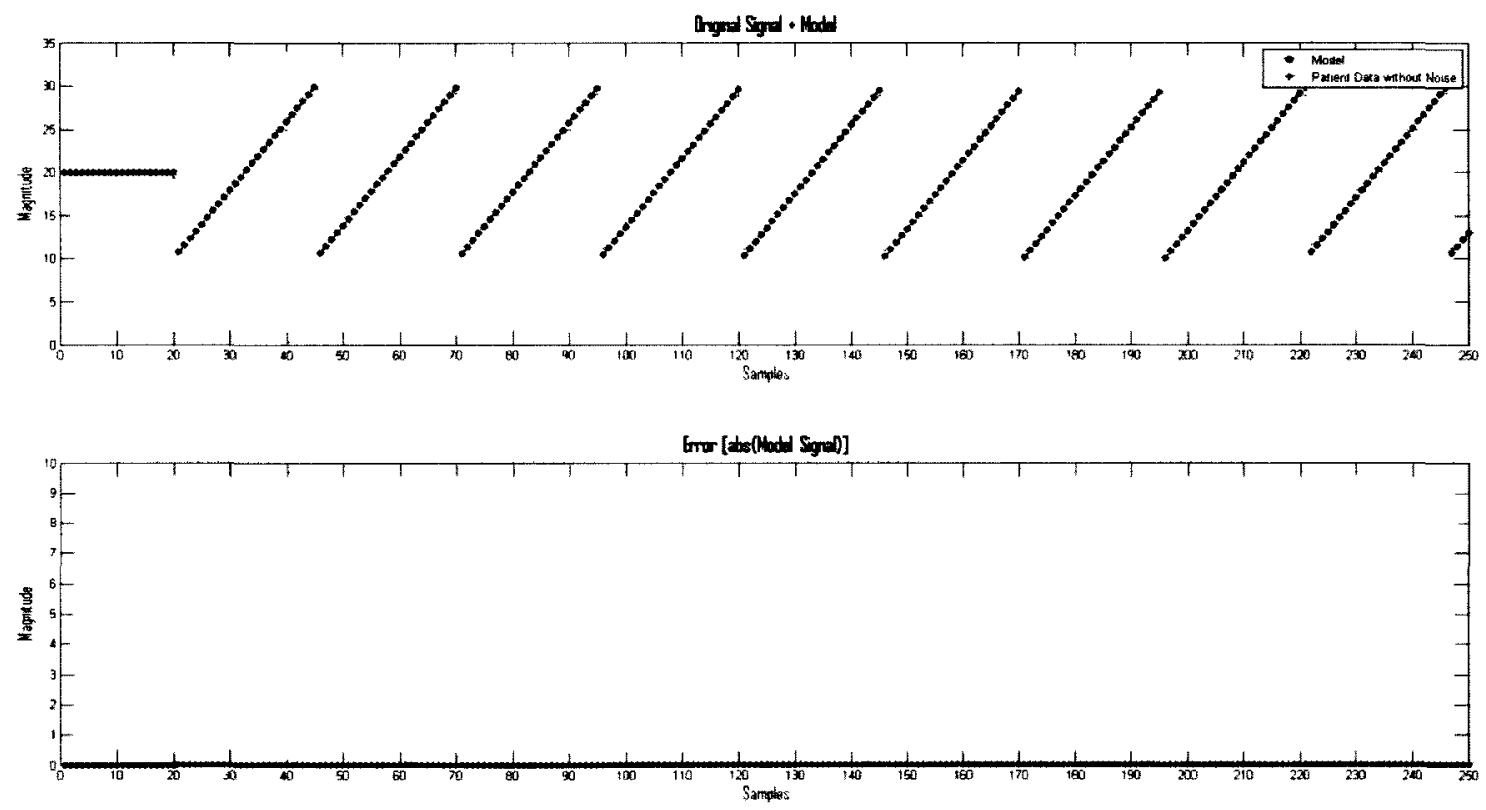

Figure B.4: Plot 4 


\section{Appendix C: Segmentation Algorithm - Varying Noise Level Plots}
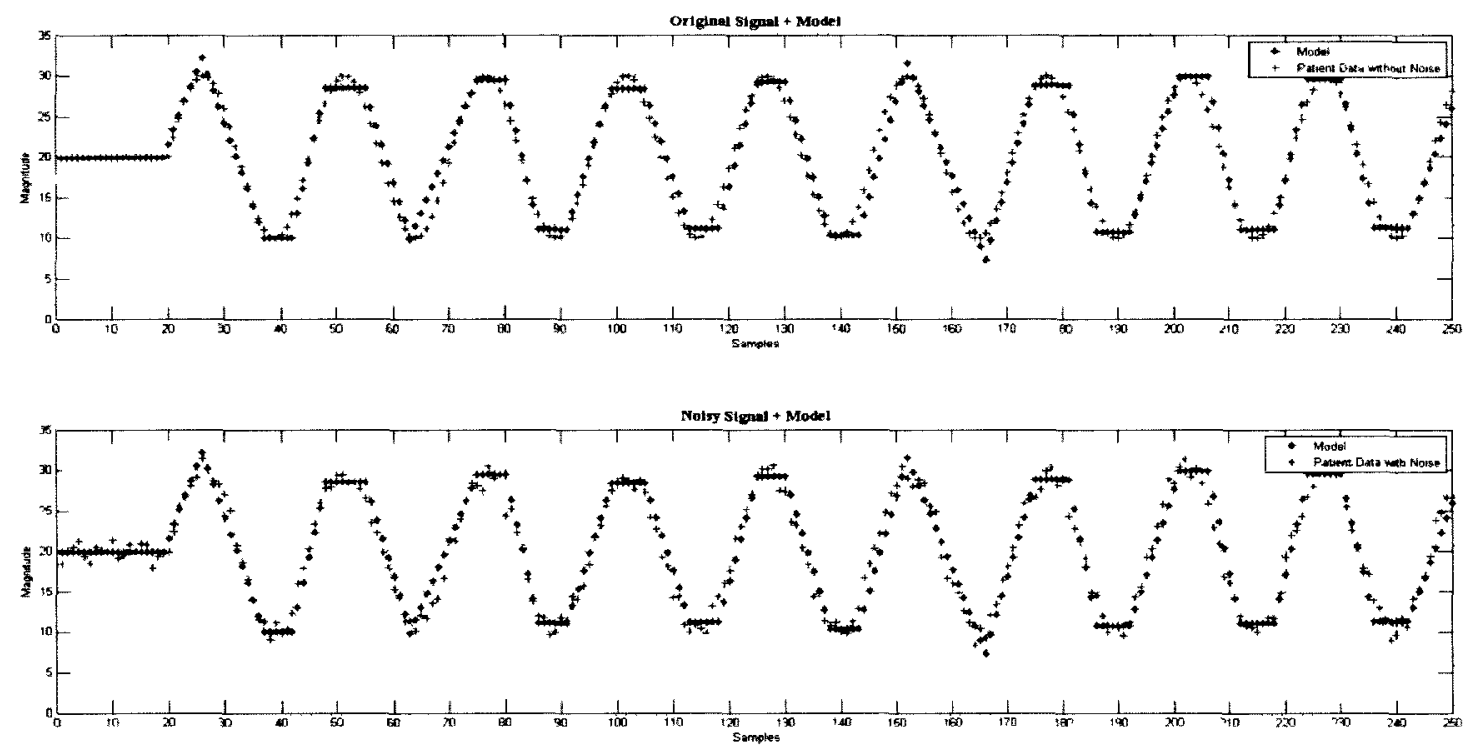

Figure C.1: Plot 1 - Noise 3\%
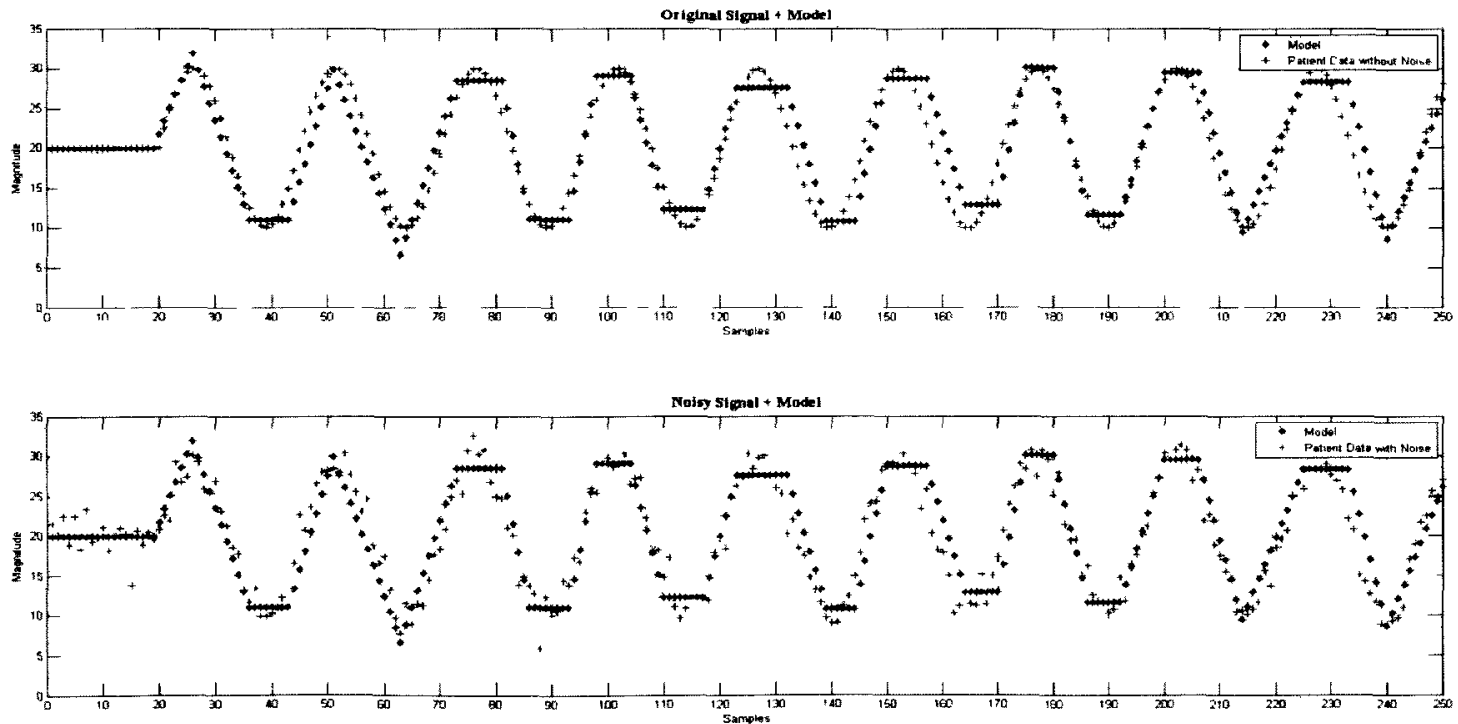

Figure C.2: Plot 1 - Noise 5\% 

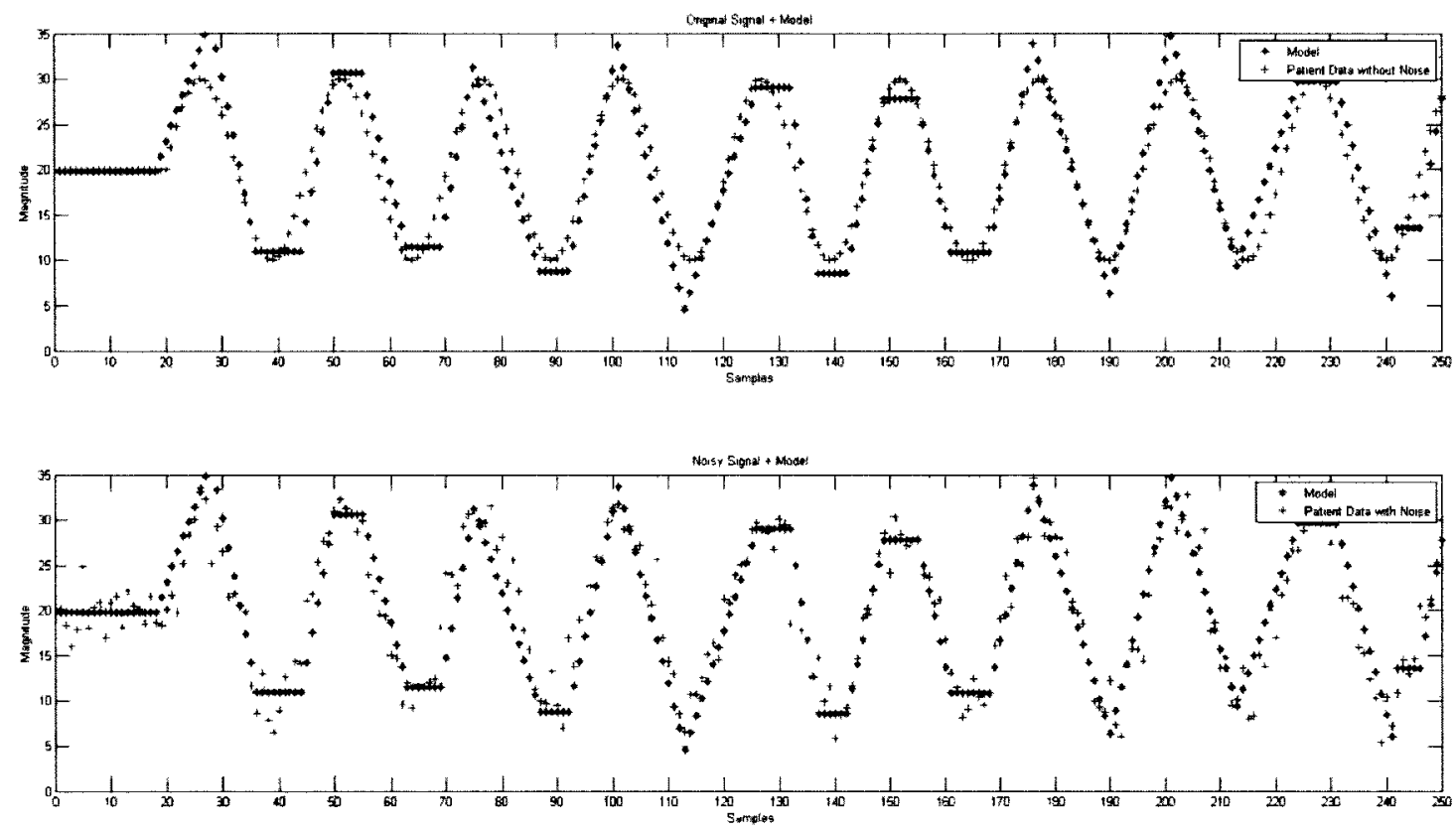

Figure C.3: Plot 1 - Noise 7\%
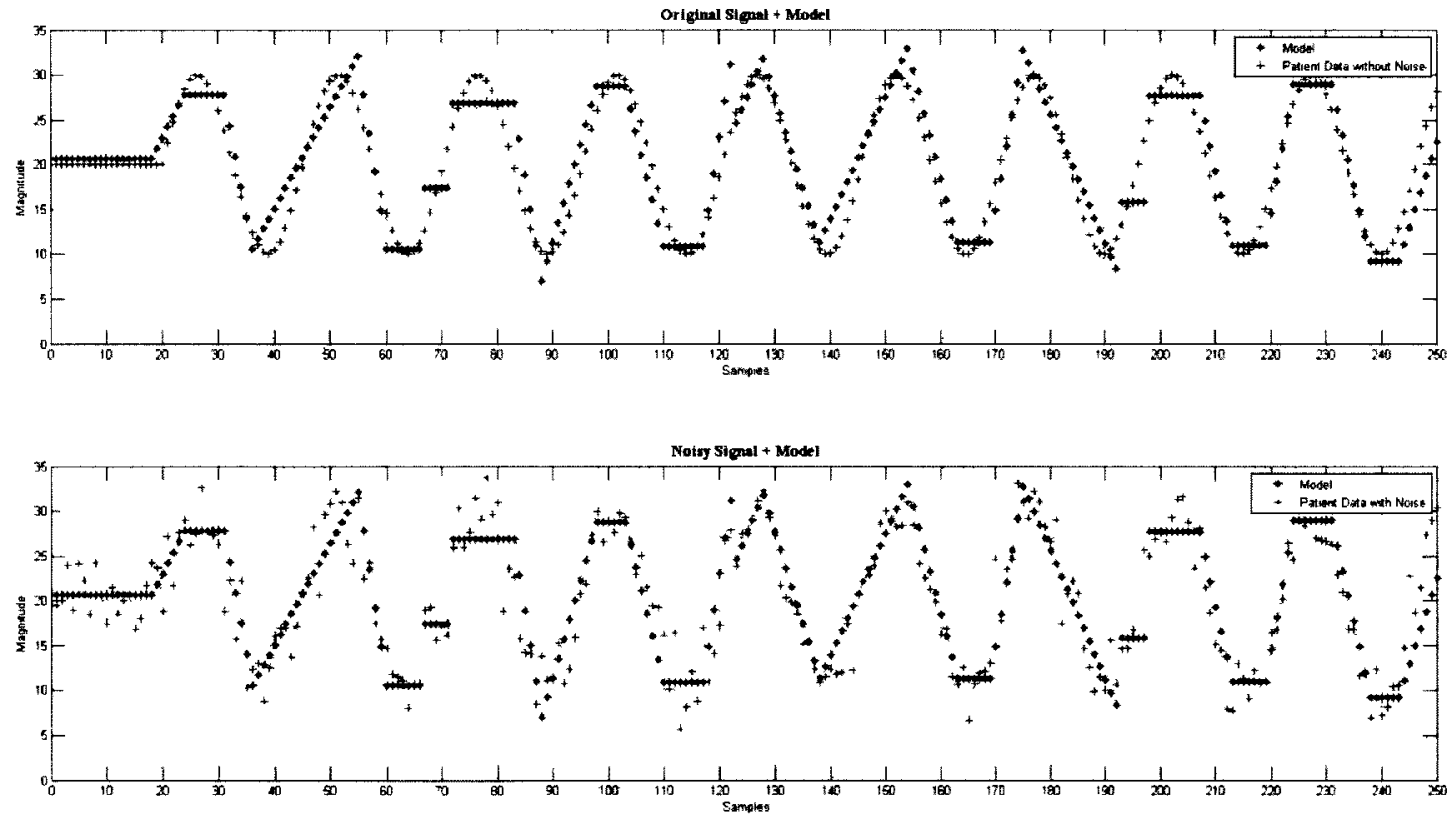

Figure C.4: Plot 1 - Noise 9\% 

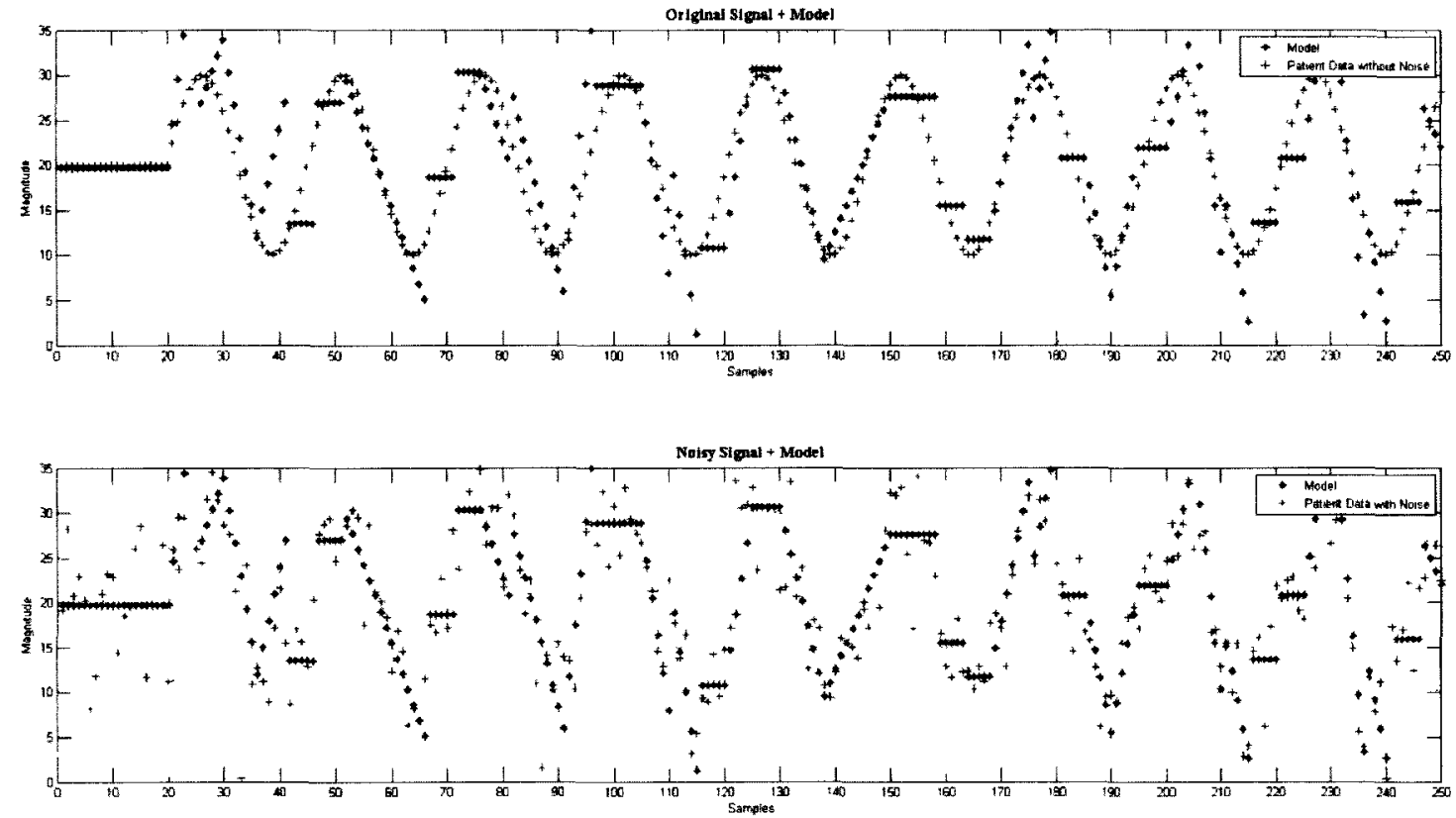

Figure C.5: Plot 1 - Noise 17\%
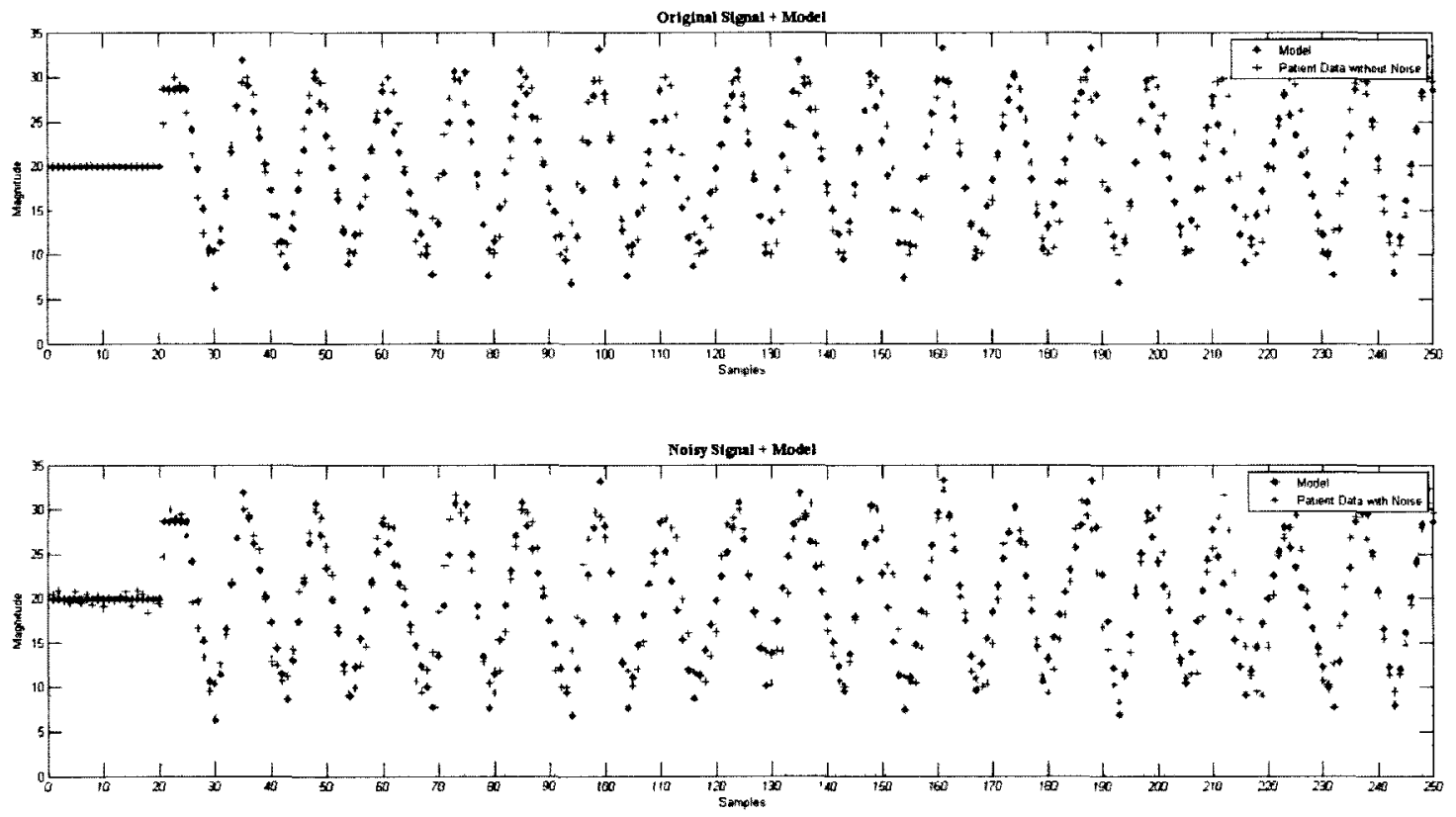

Figure C.6: Plot 2 - Noise 3\% 

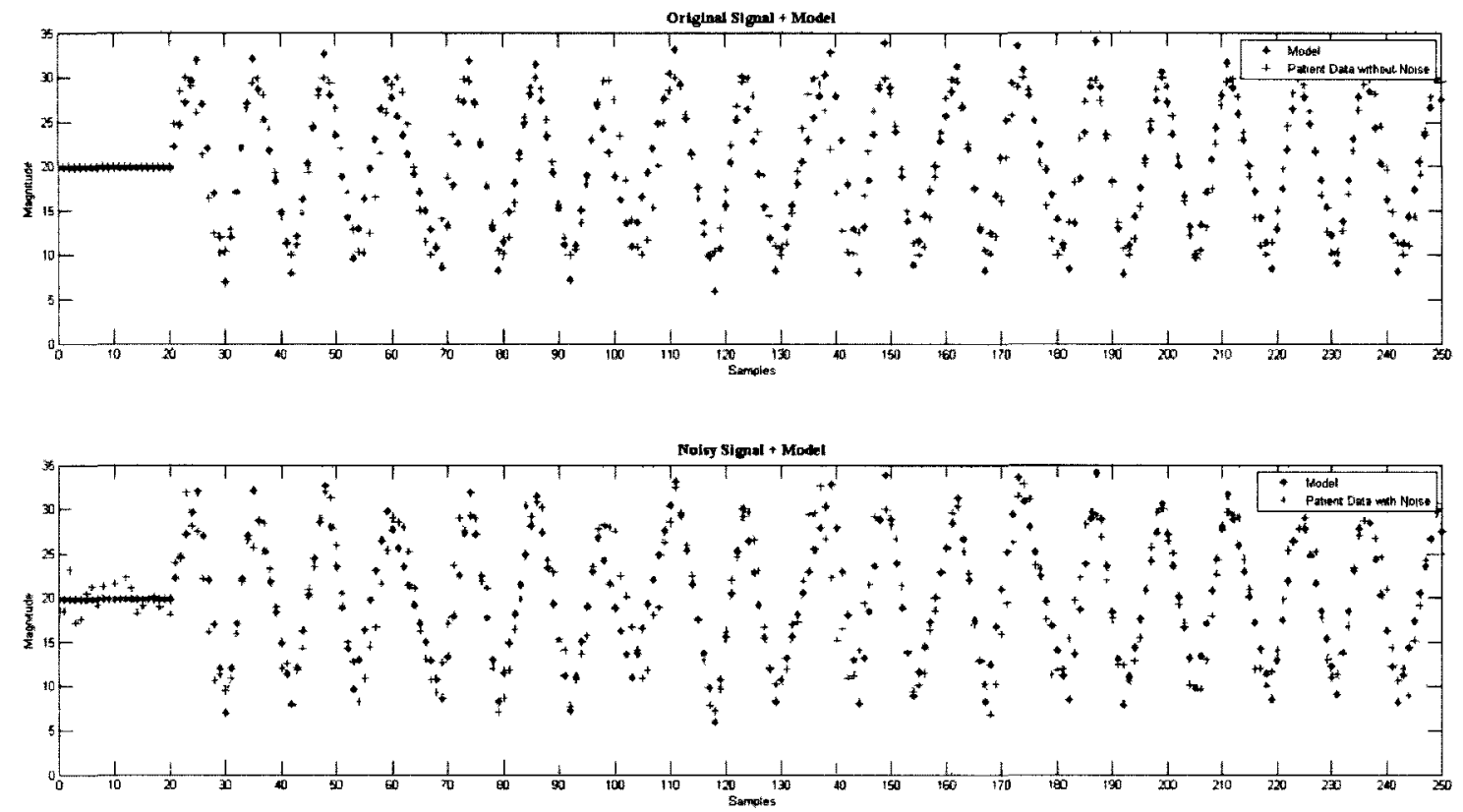

Figure C.7: Plot 2 - Noise 5\%
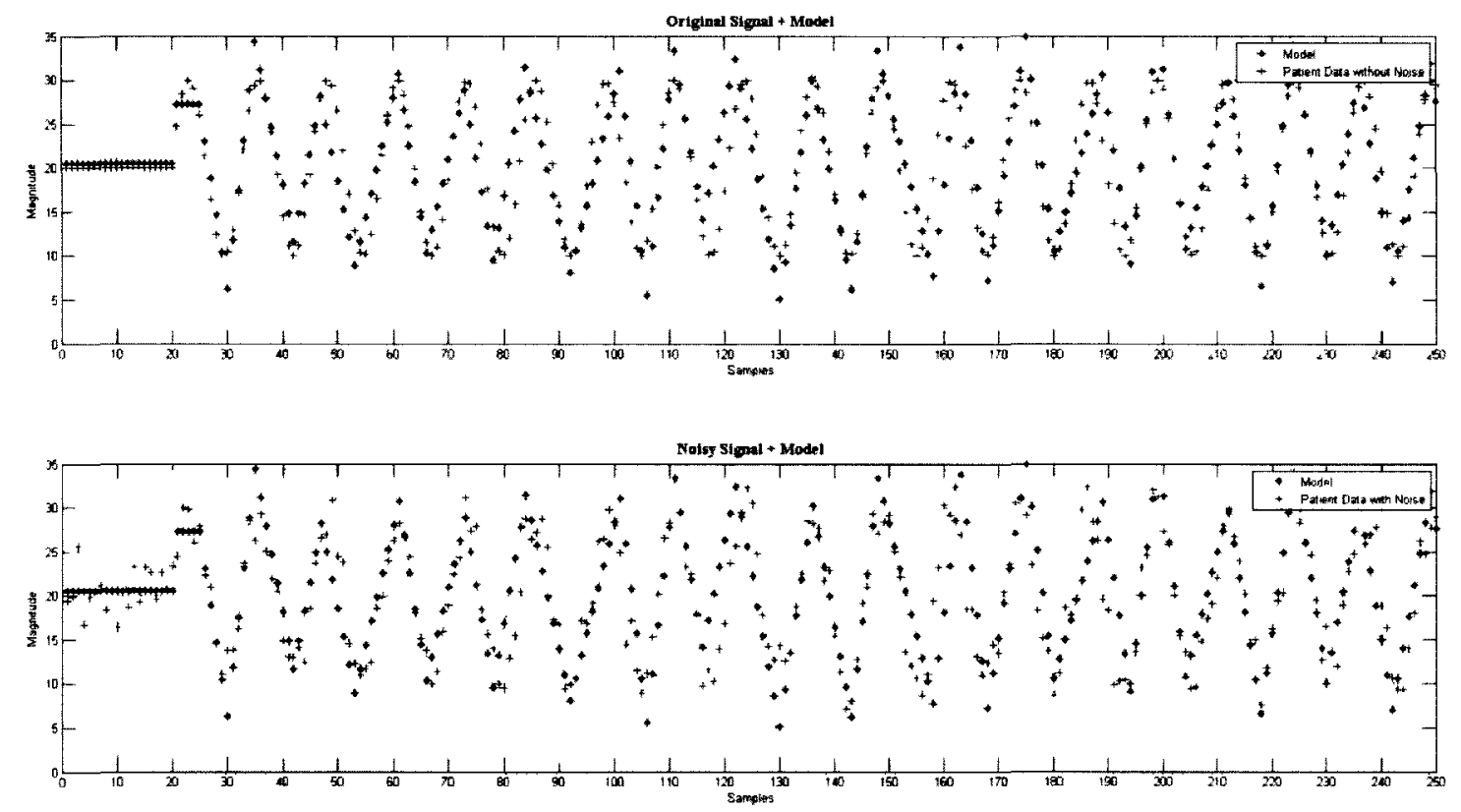

Figure C.8: Plot 2 - Noise 7\% 

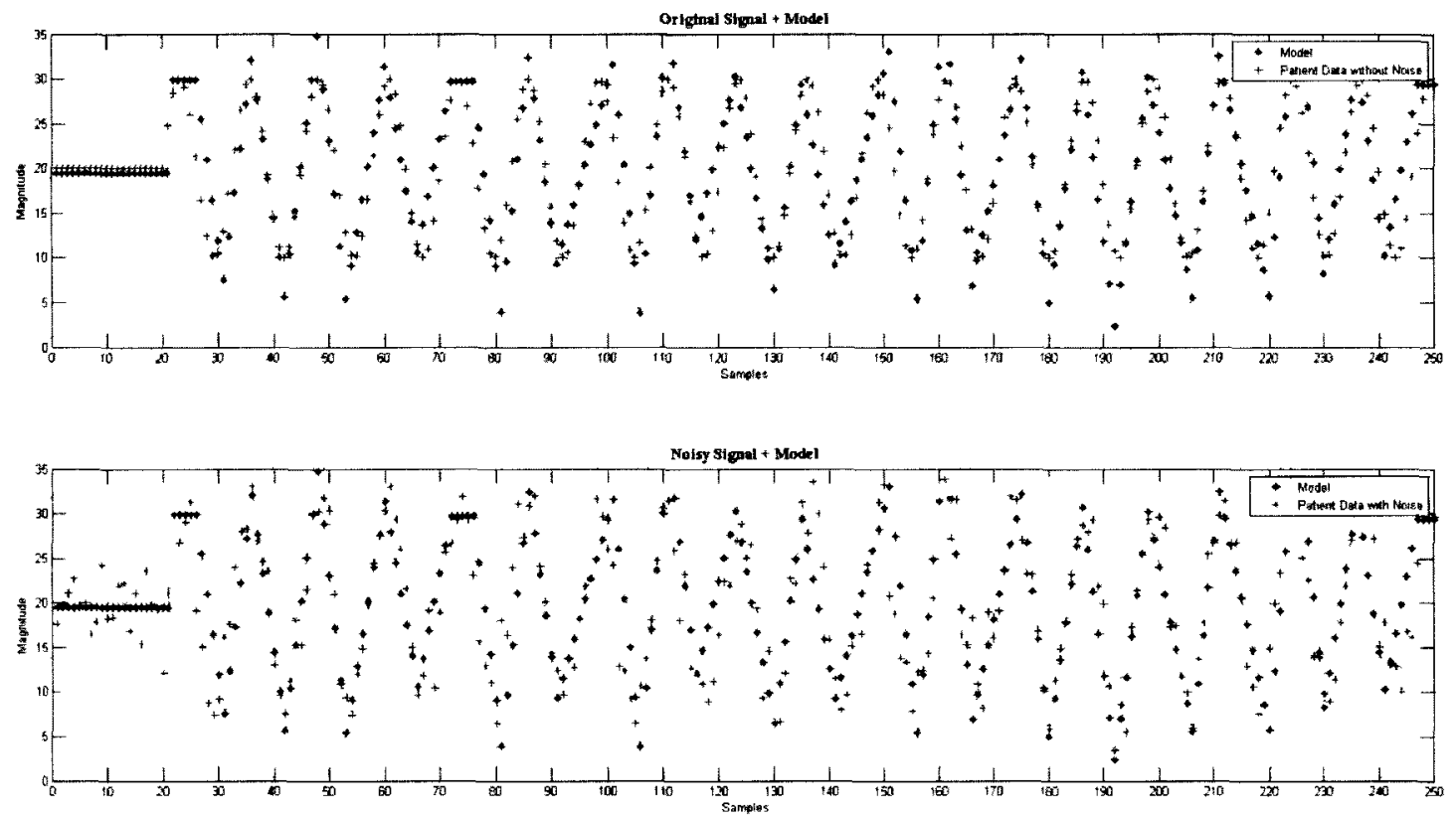

Figure C.9: Plot 2 - Noise 9\%
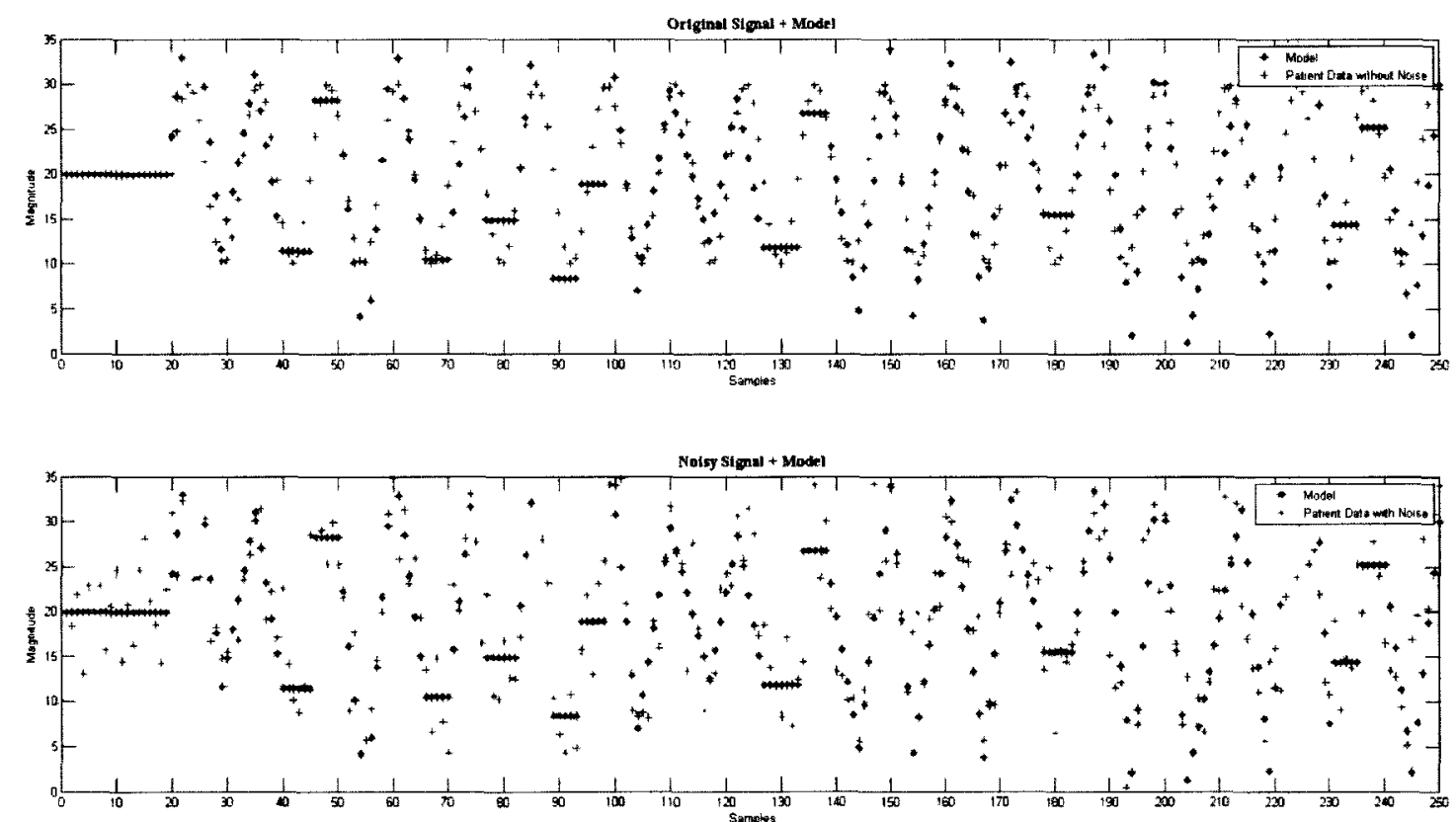

Figure C.10: Plot 2 - Noise 17\% 

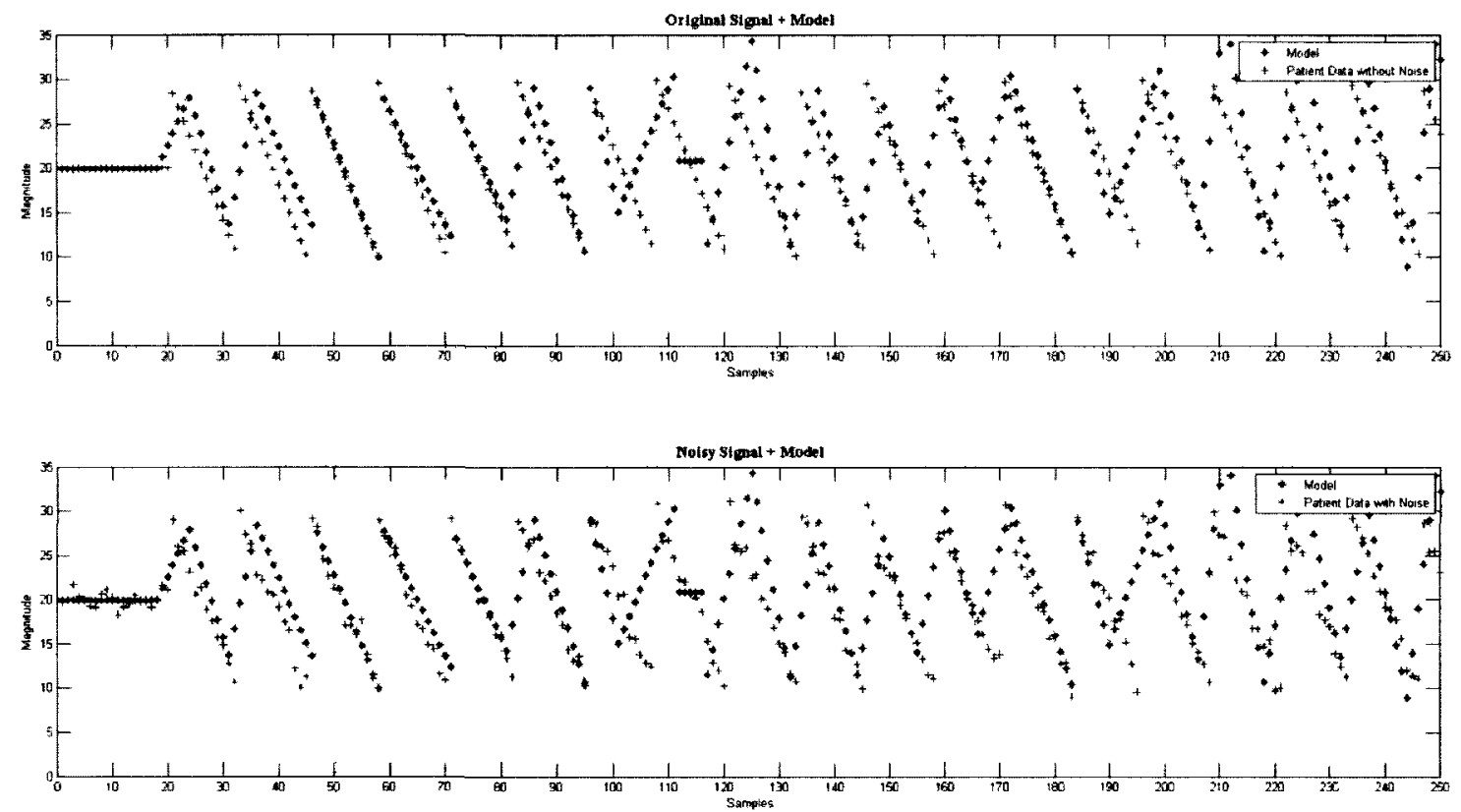

Figure C.11: Plot 3 - Noise 3\%
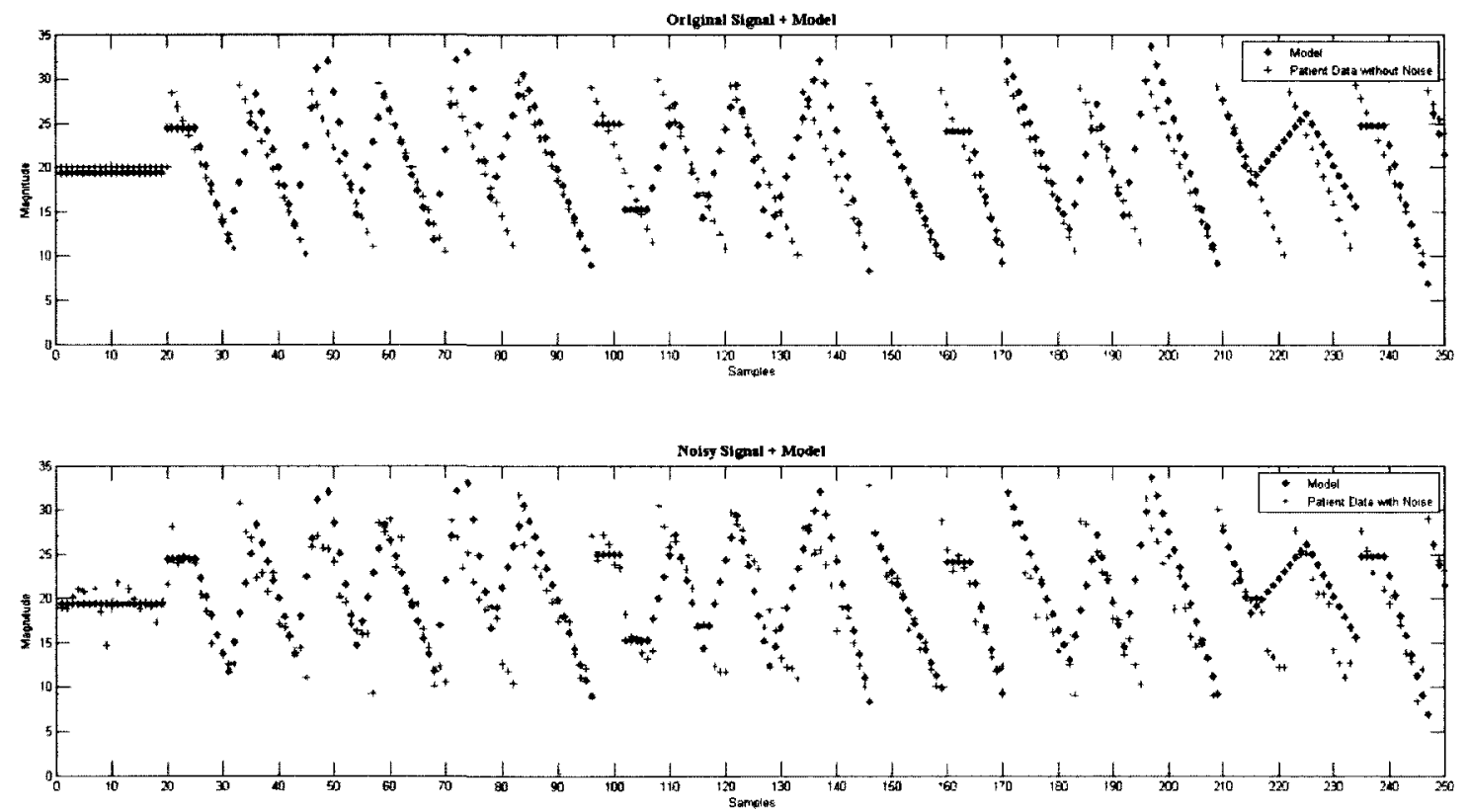

Figure C.12: Plot 3 - Noise 5\% 

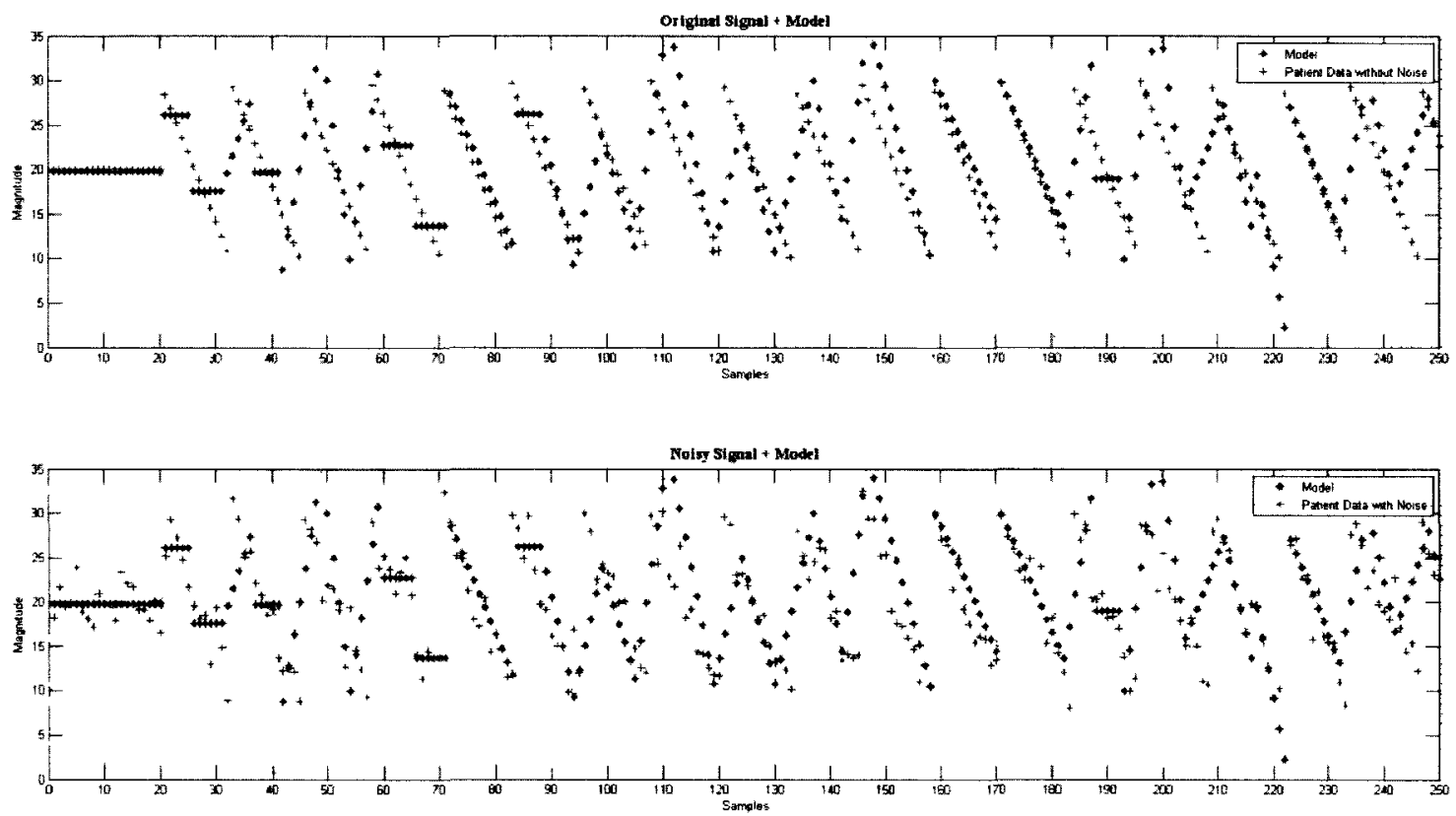

Figure C.13: Plot 3 - Noise 7\%
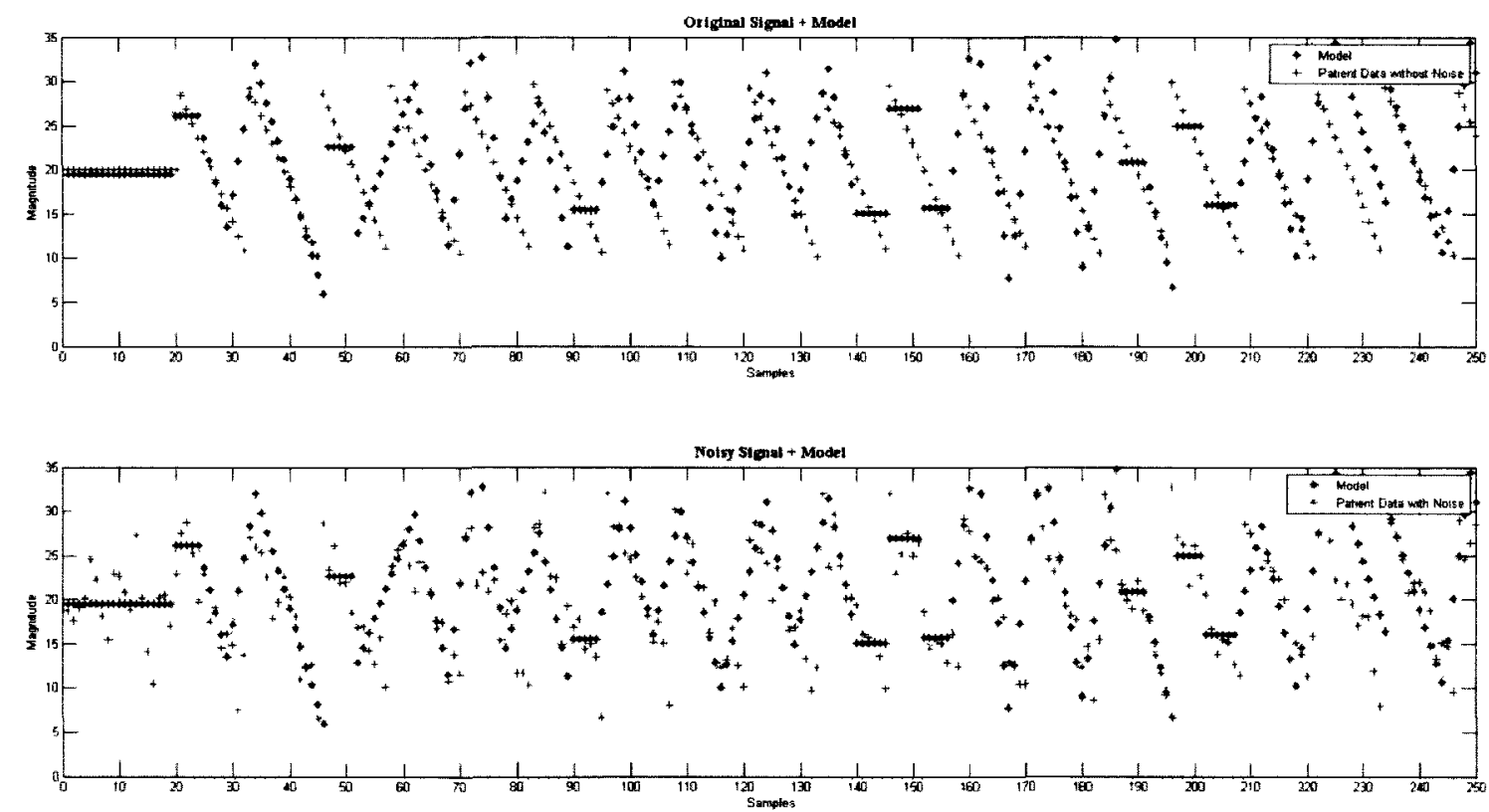

Figure C.14: Plot 3 - Noise 9\% 

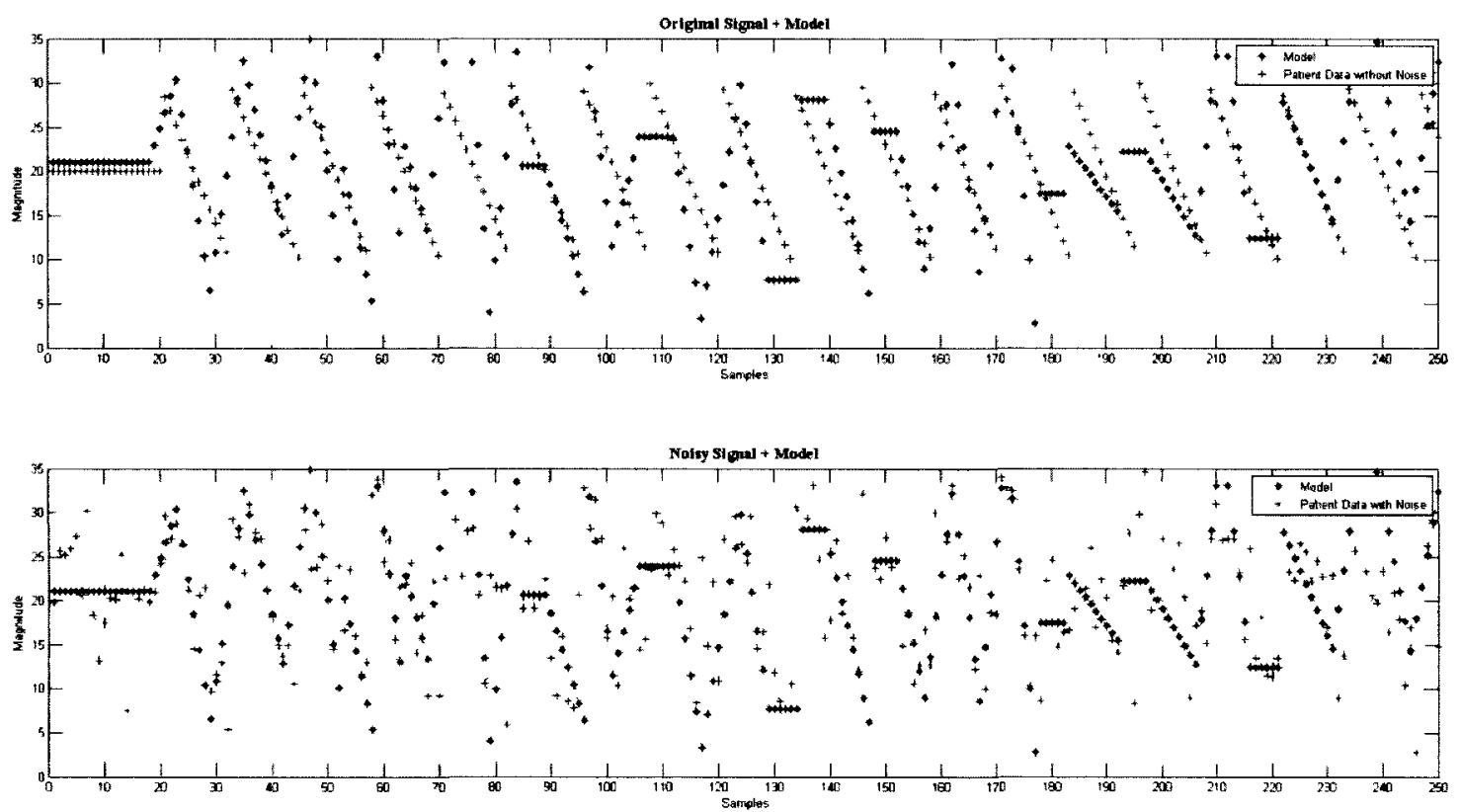

Figure C.15: Plot 3 - Noise 17\%
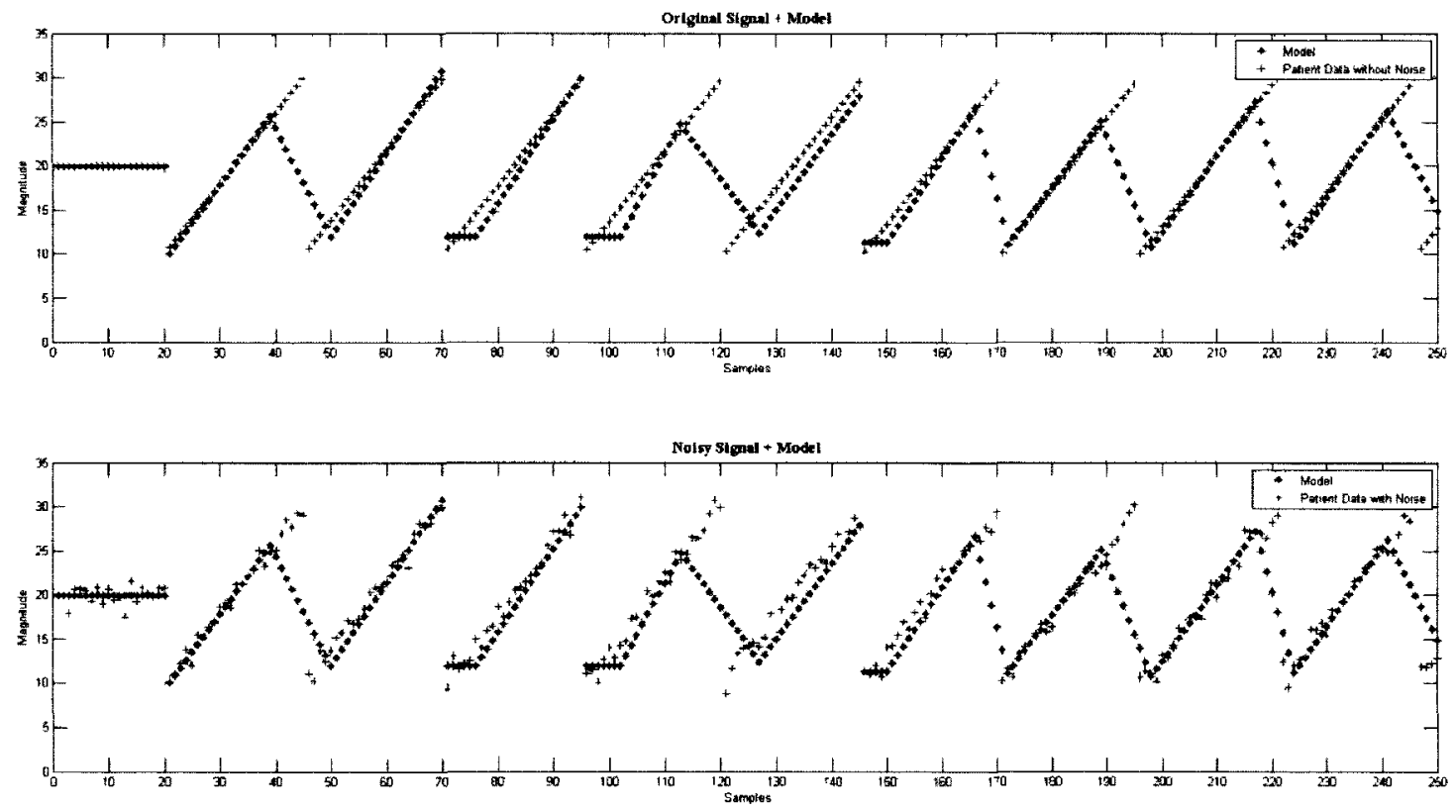

Figure C.16: Plot 4 - Noise 3\% 

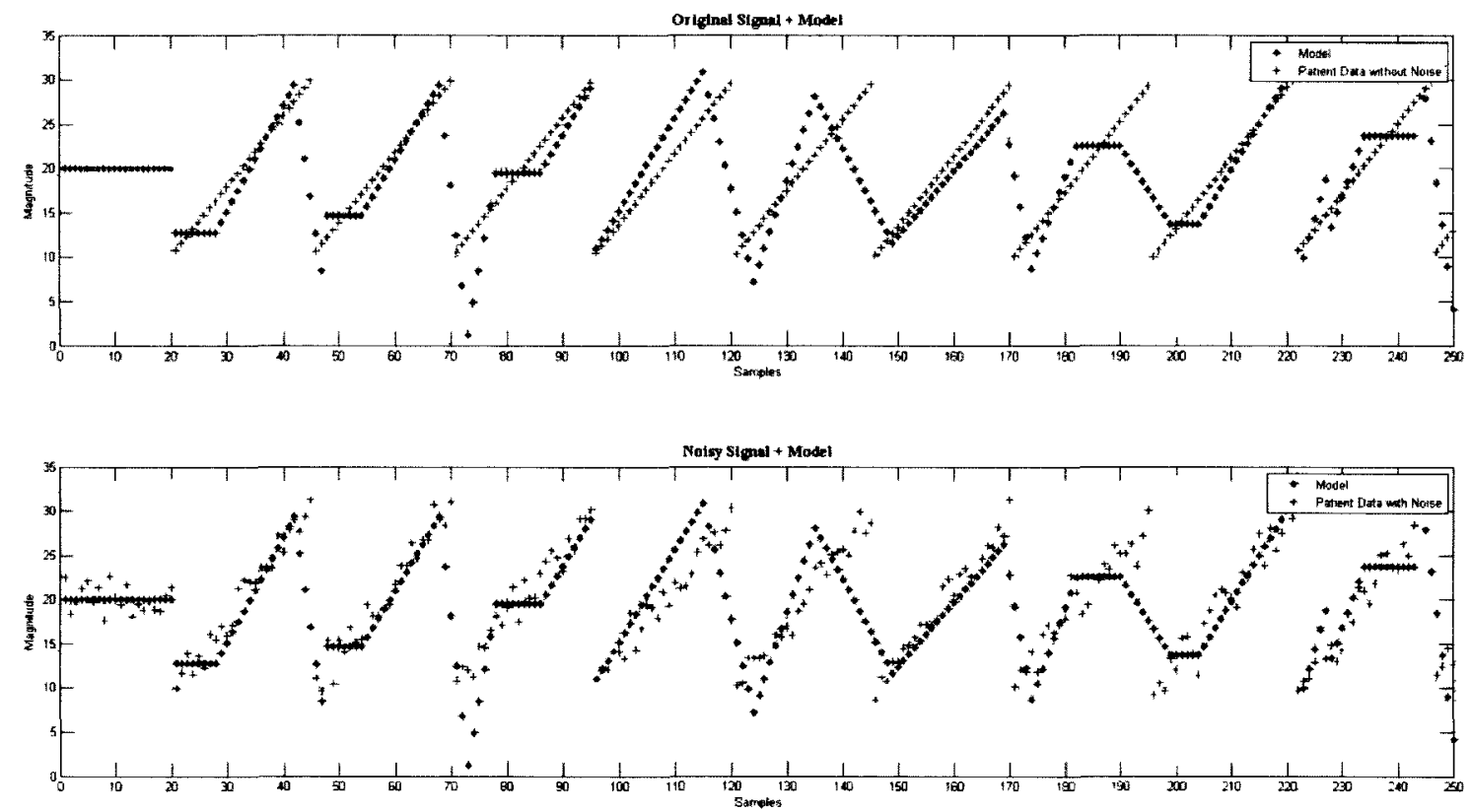

Figure C.17: Plot 4-Noise 5\%
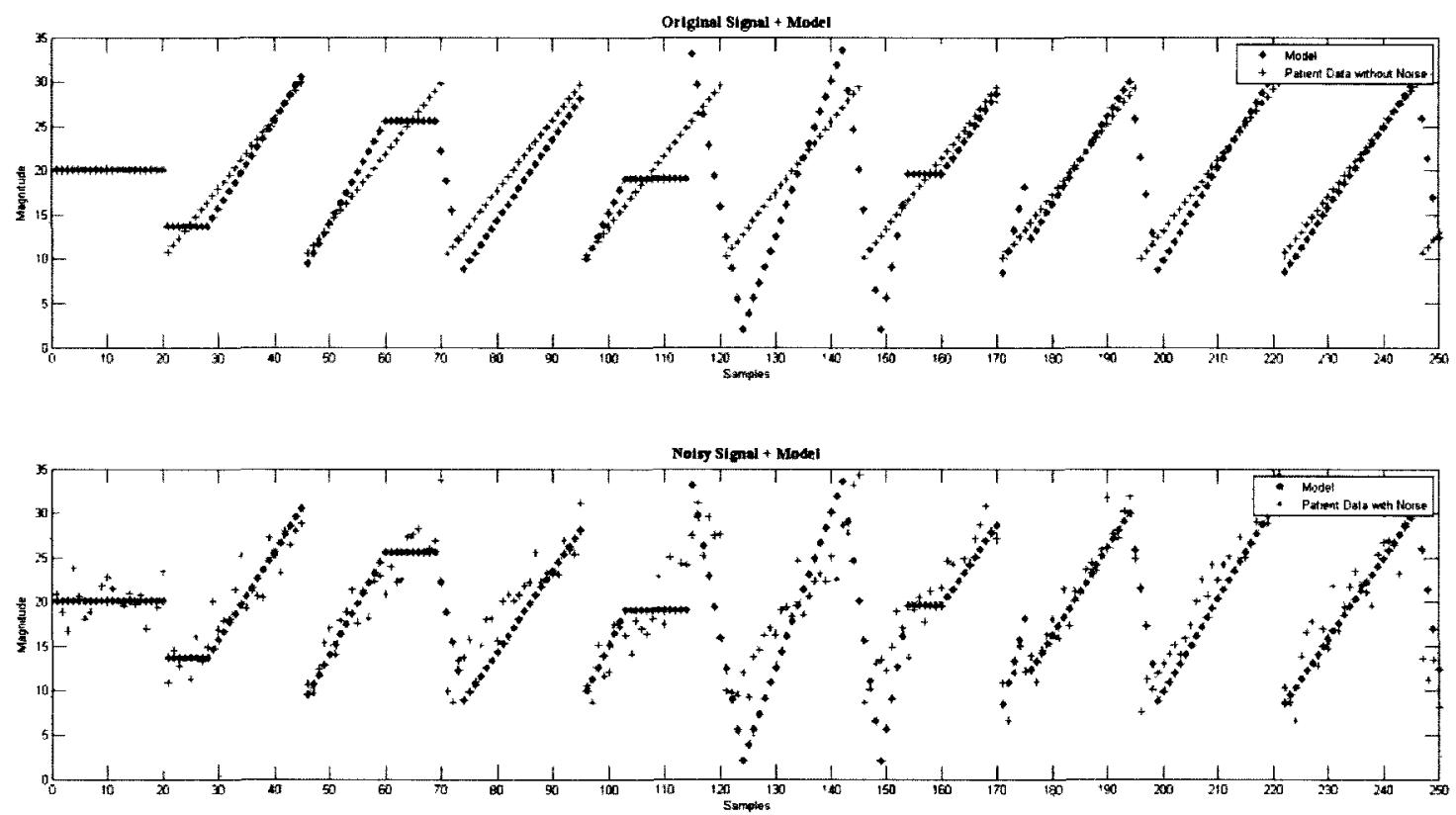

Figure C.18: Plot 4-Noise 7\% 

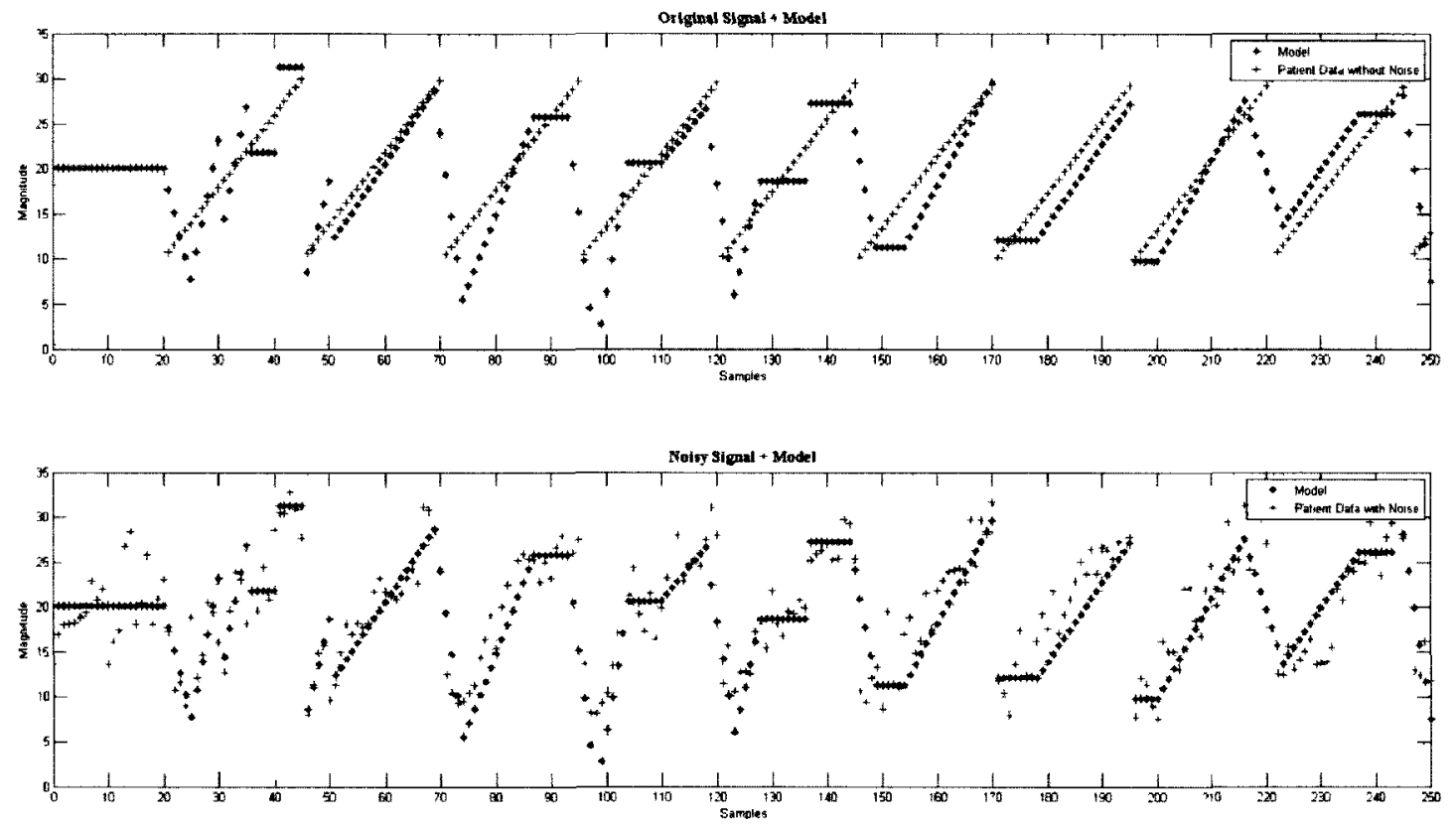

Figure C.19: Plot 4 - Noise 9\%
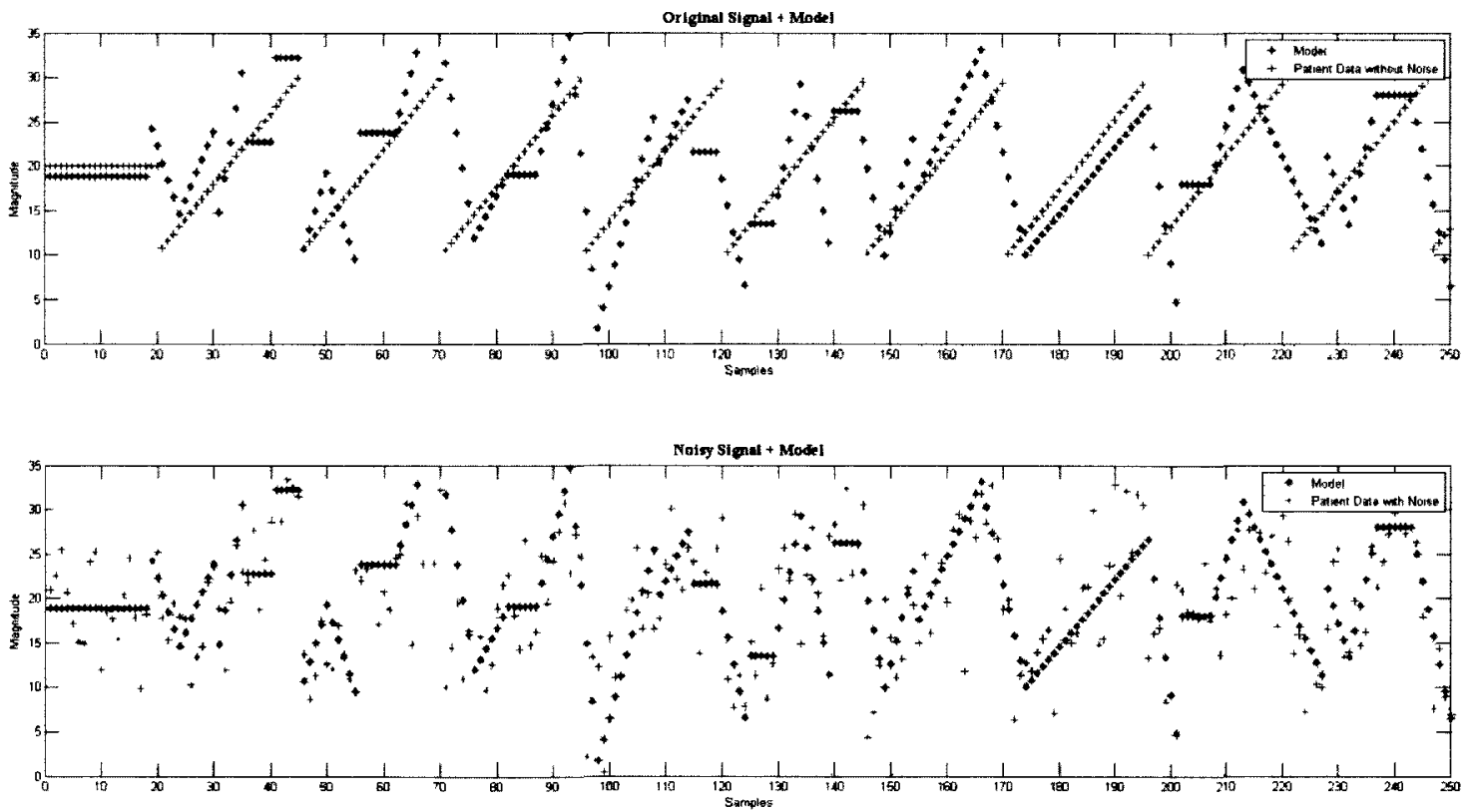

Figure C.20: Plot 4 - Noise 17\% 


\section{Appendix D: Feed-forward Neural Network - Optimal Parameter Plots}
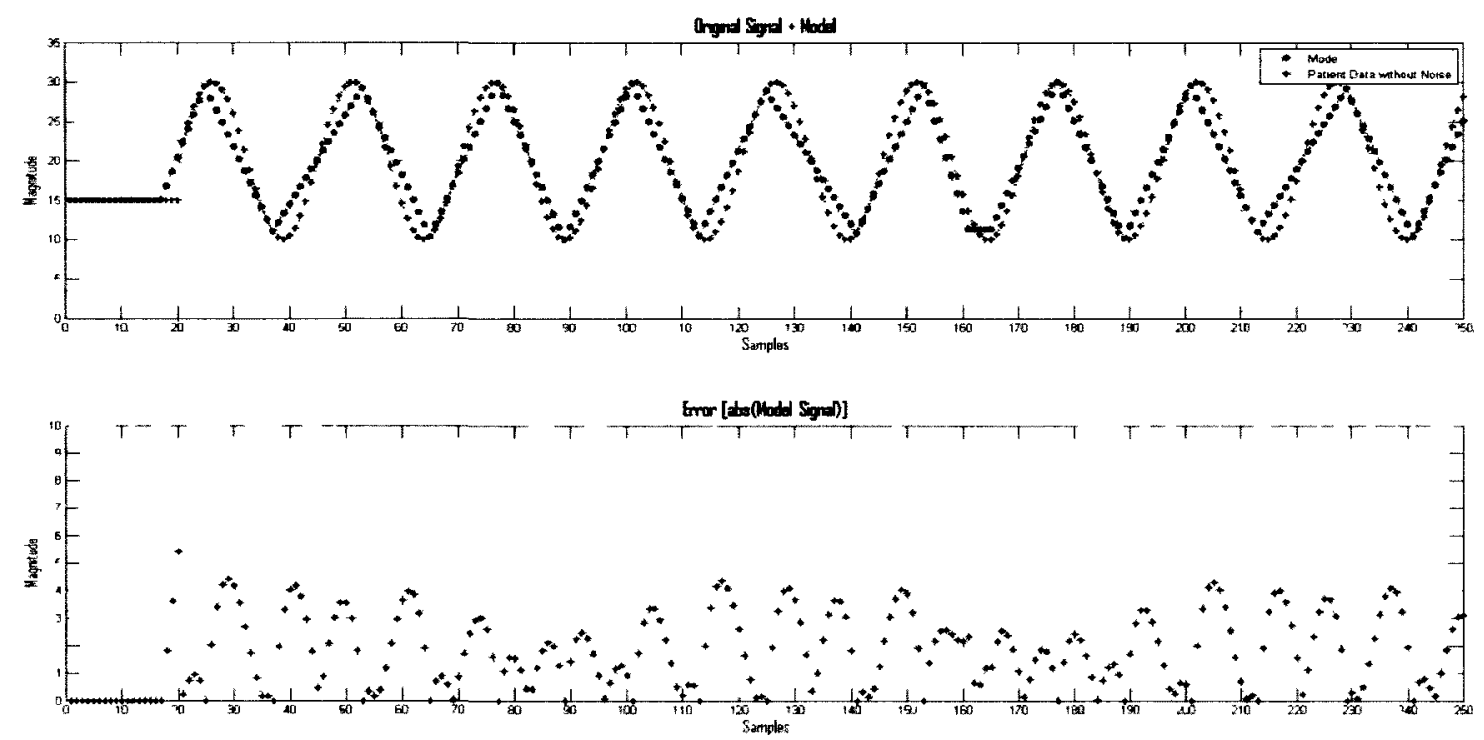

Figure D.1: Plot 1
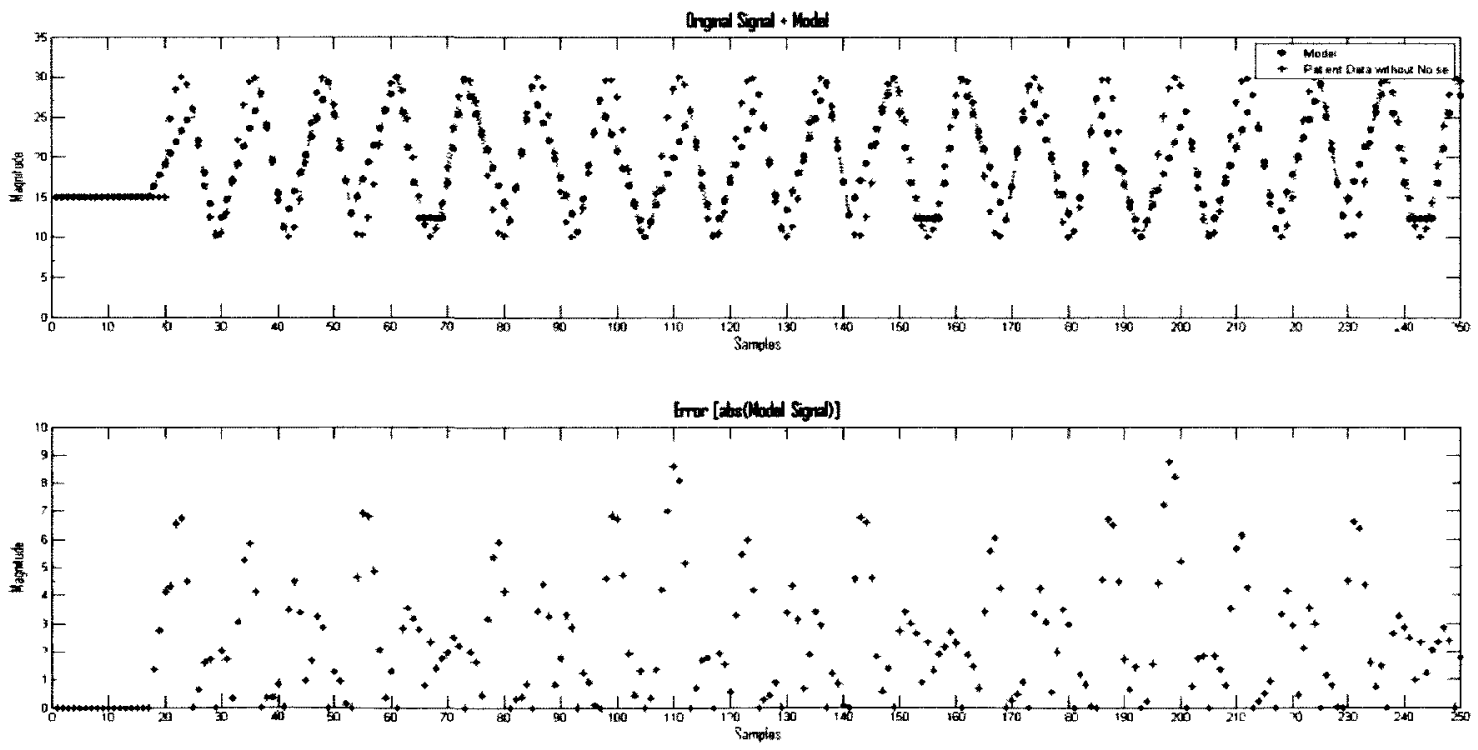

Figure D.2: Plot 2 

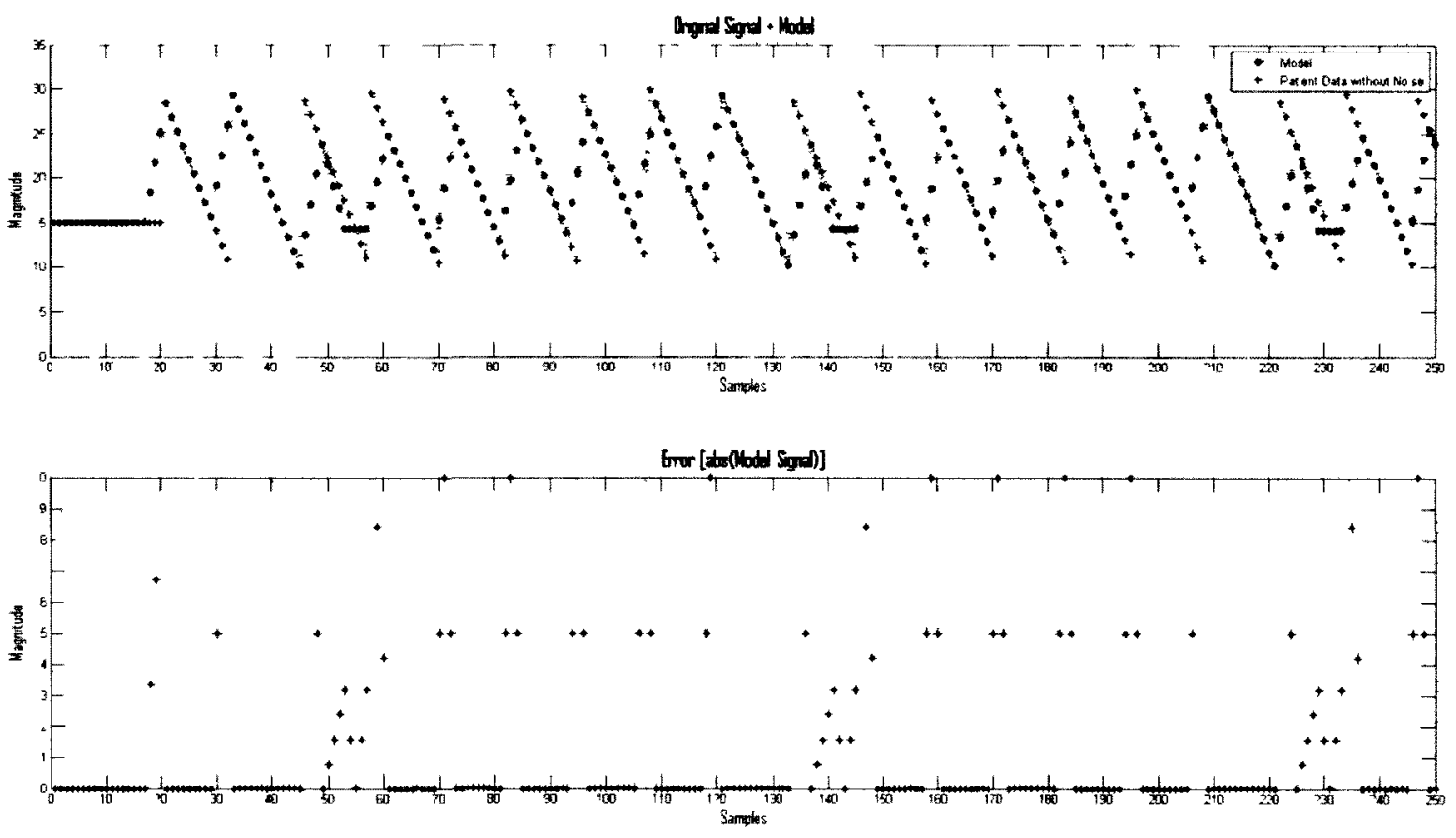

Figure D.3: Plot 3
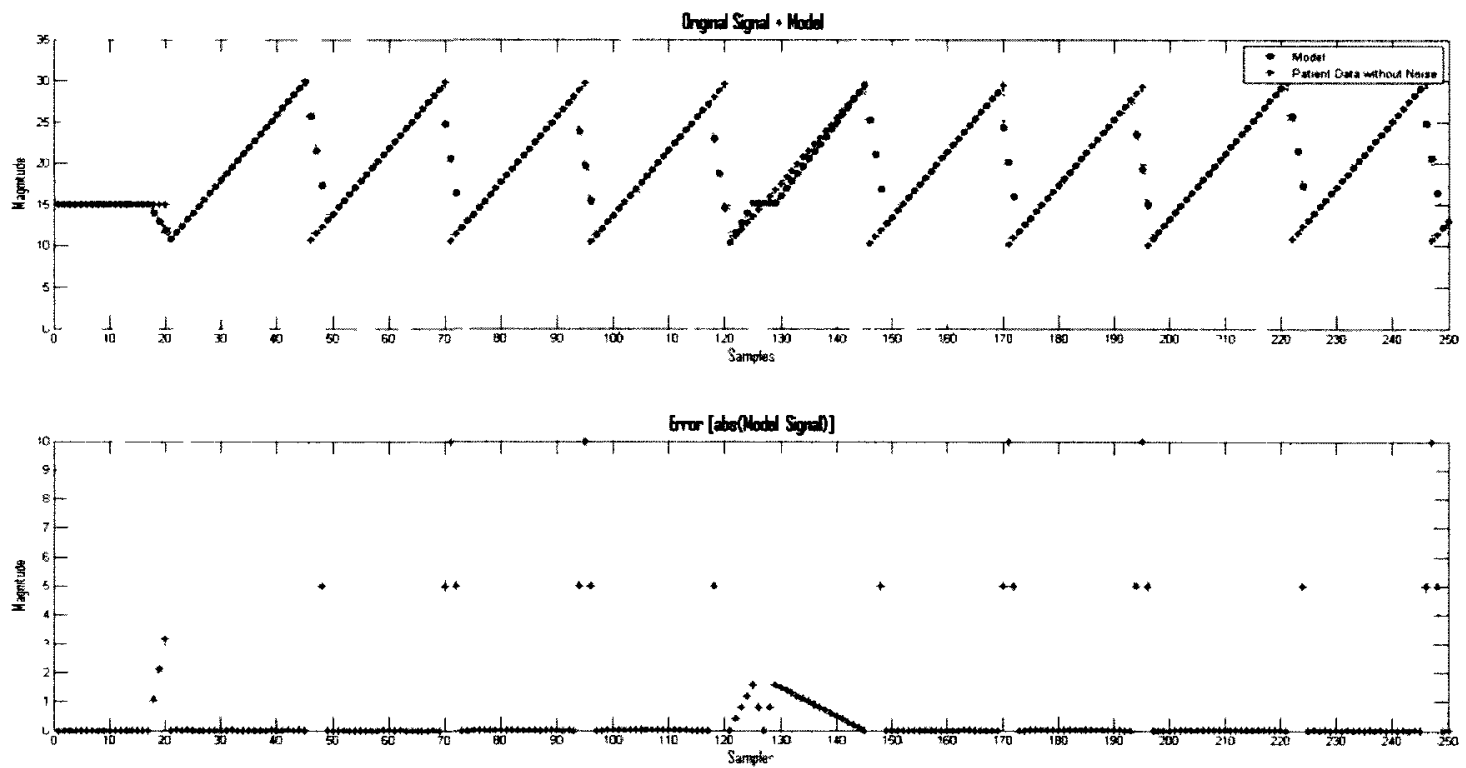

Figure D.4: Plot 4 


\section{Appendix E: Feed-forward Neural Network - Varying Noise Level Plots}
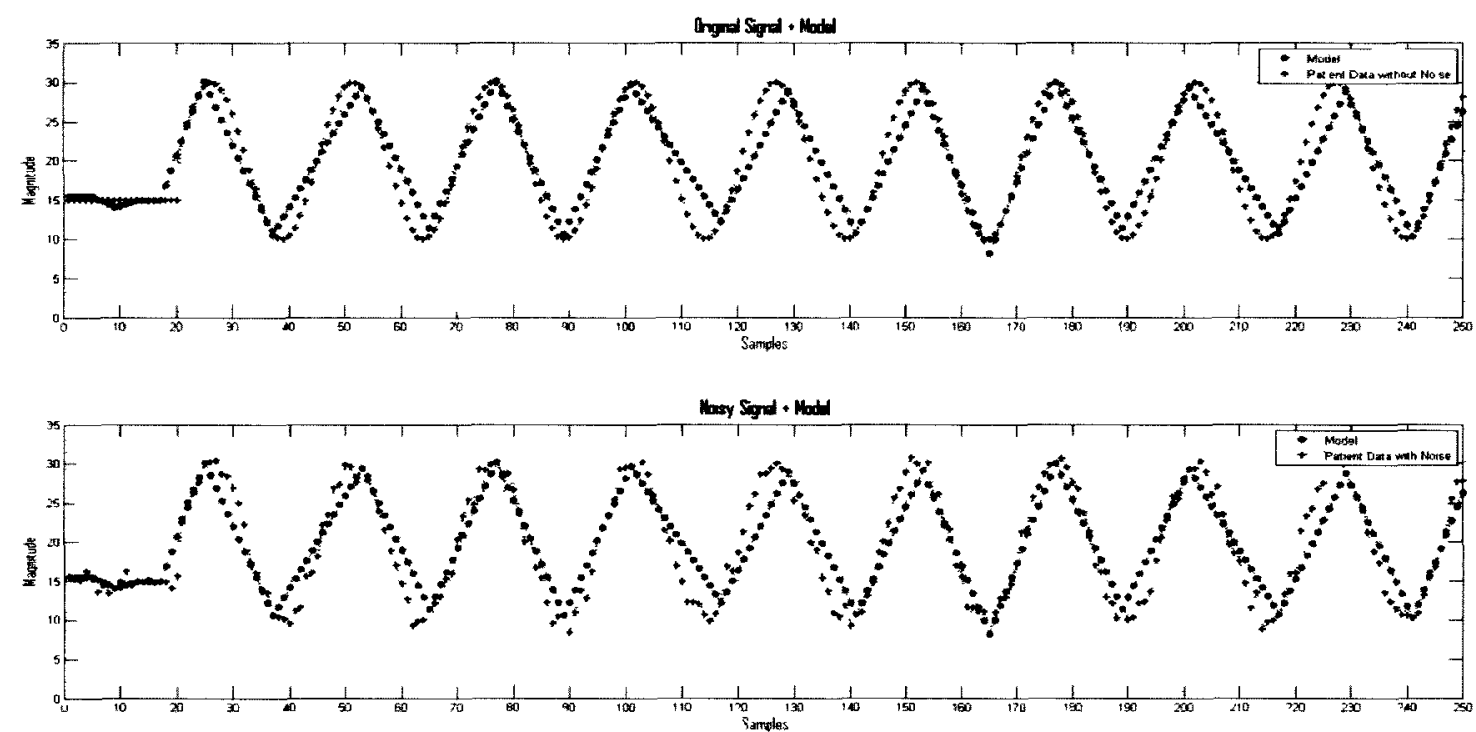

Figure E.1: Plot 1 - Noise 3\%
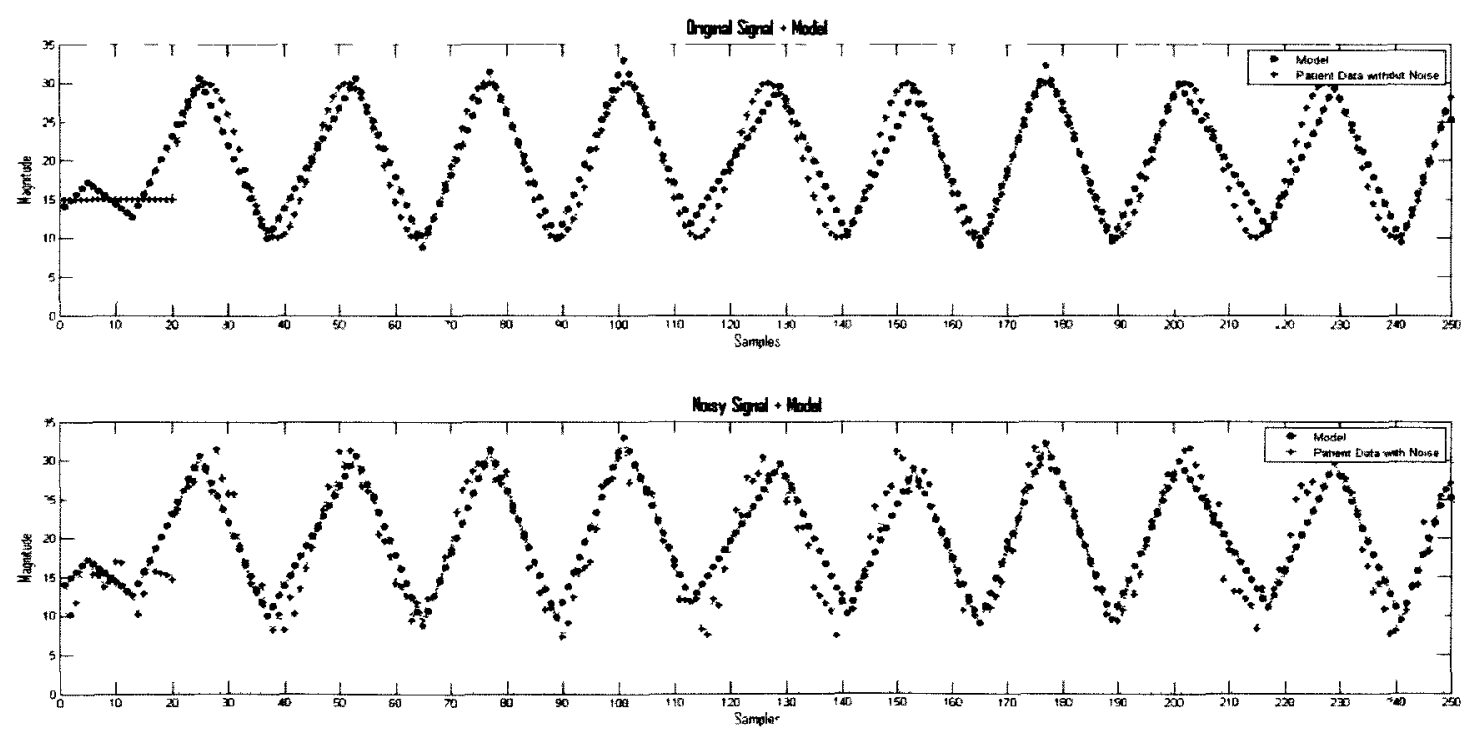

Figure E.2: Plot 1 - Noise 5\% 

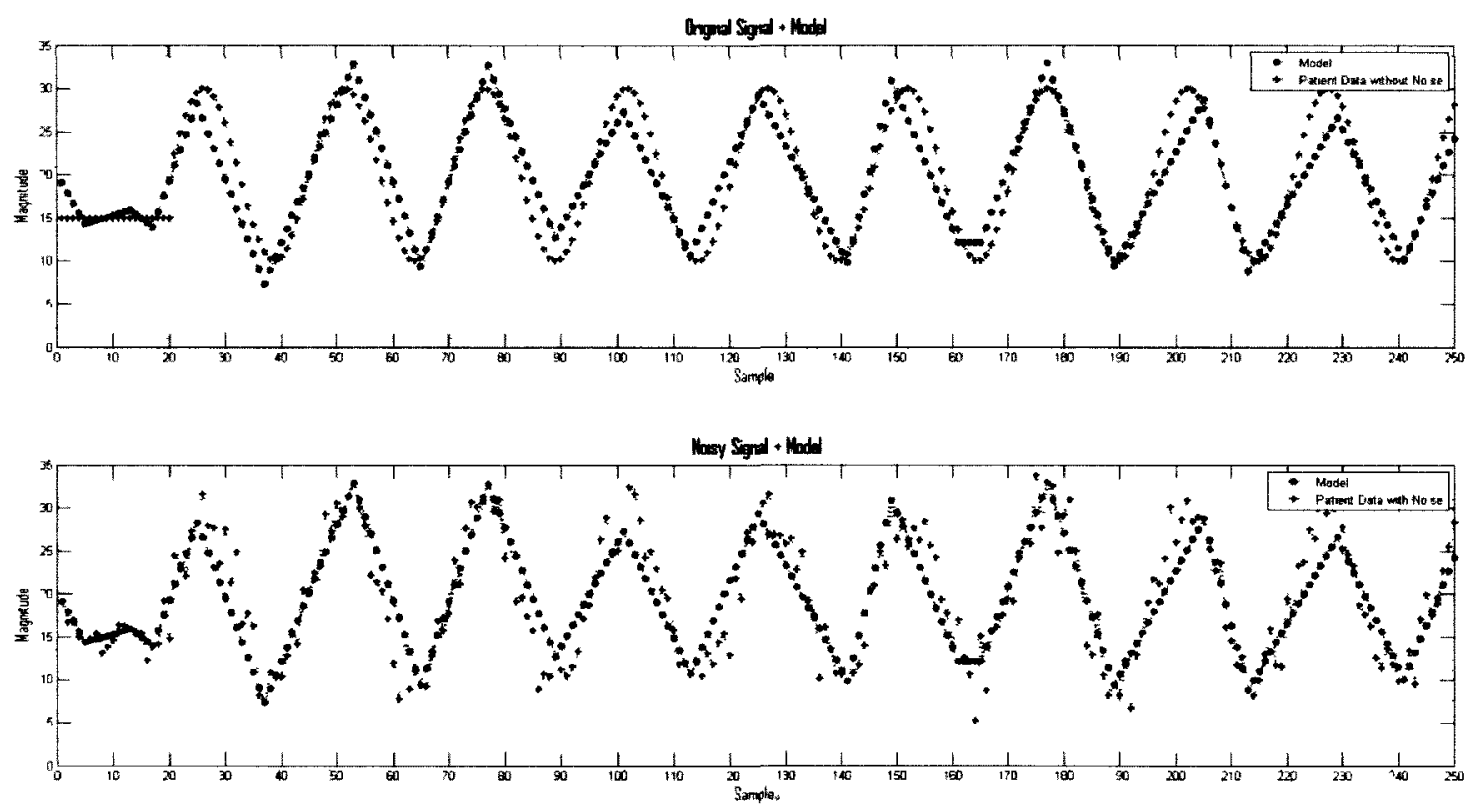

Figure E.3: Plot 1 - Noise 7\%
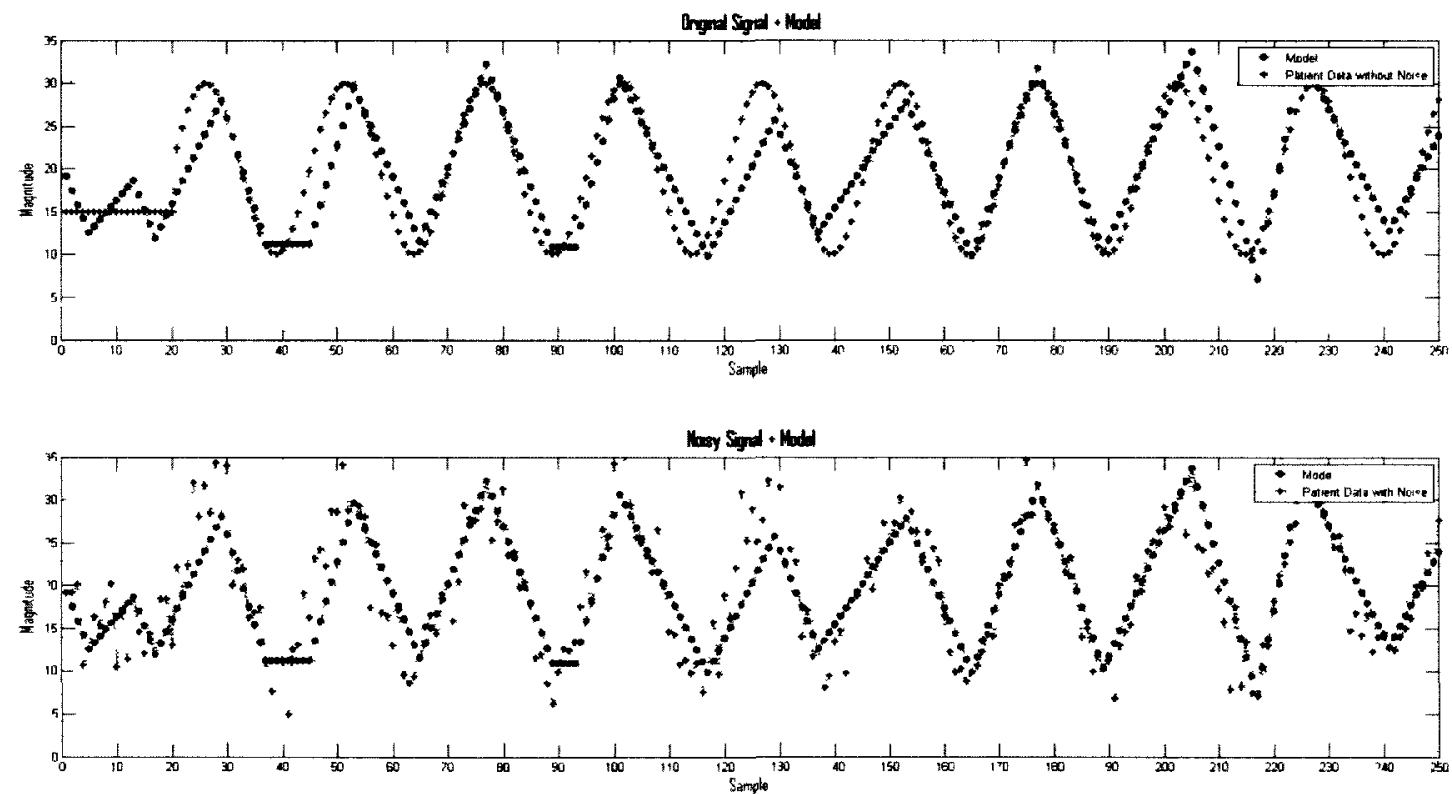

Figure E.4: Plot 1 - Noise 9\% 

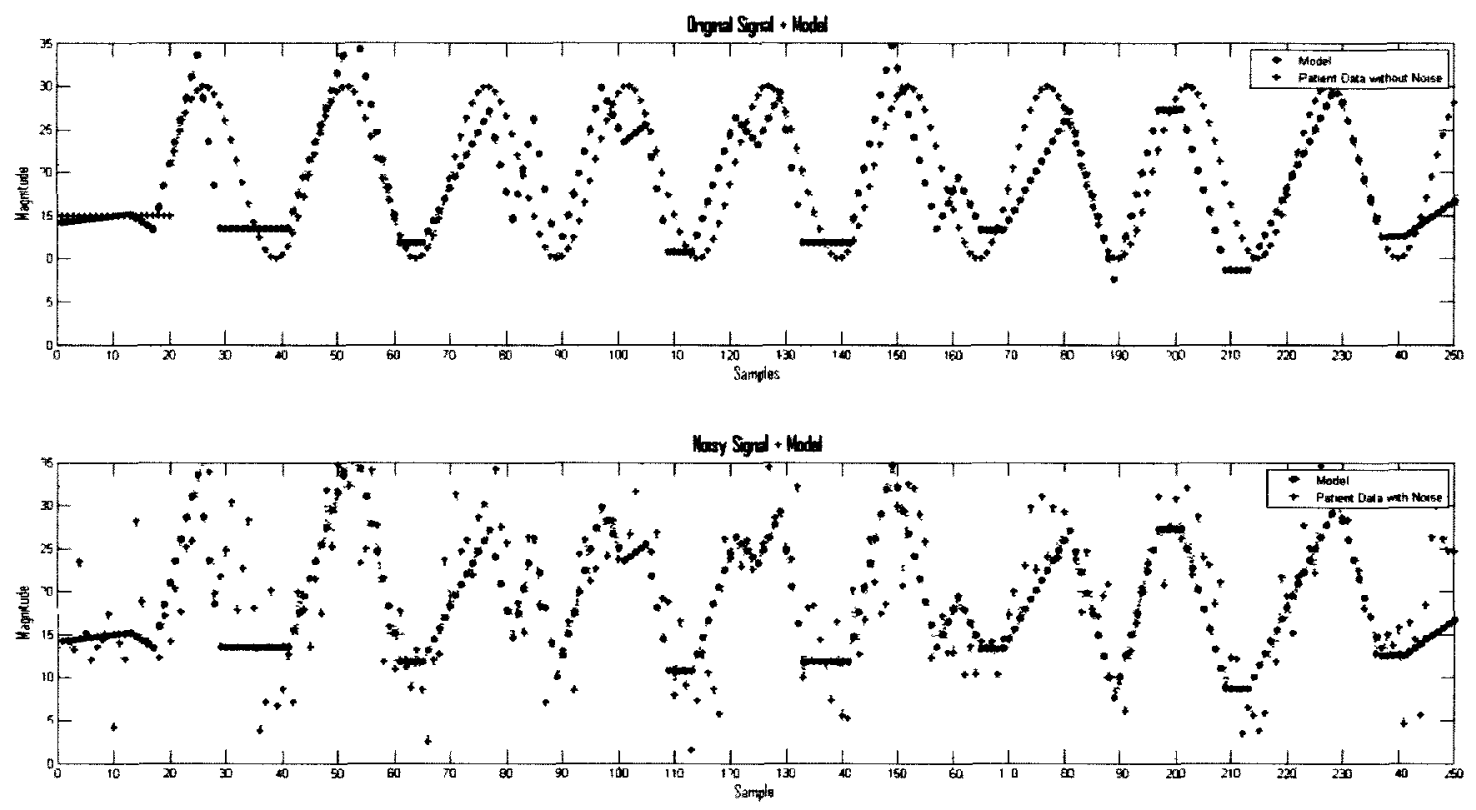

Figure E.5: Plot 1 - Noise 17\%
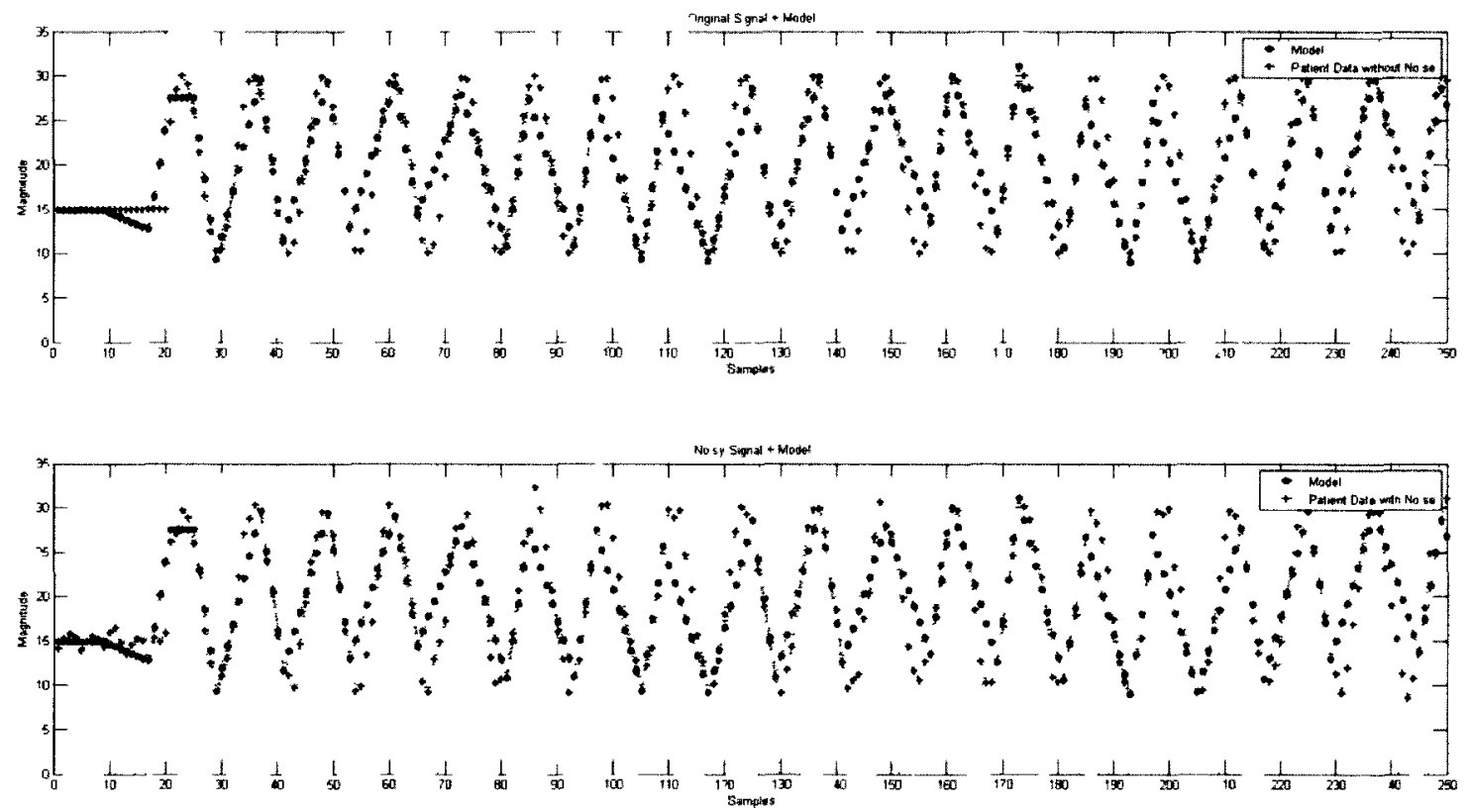

Figure E.6: Plot 2 - Noise 3\% 

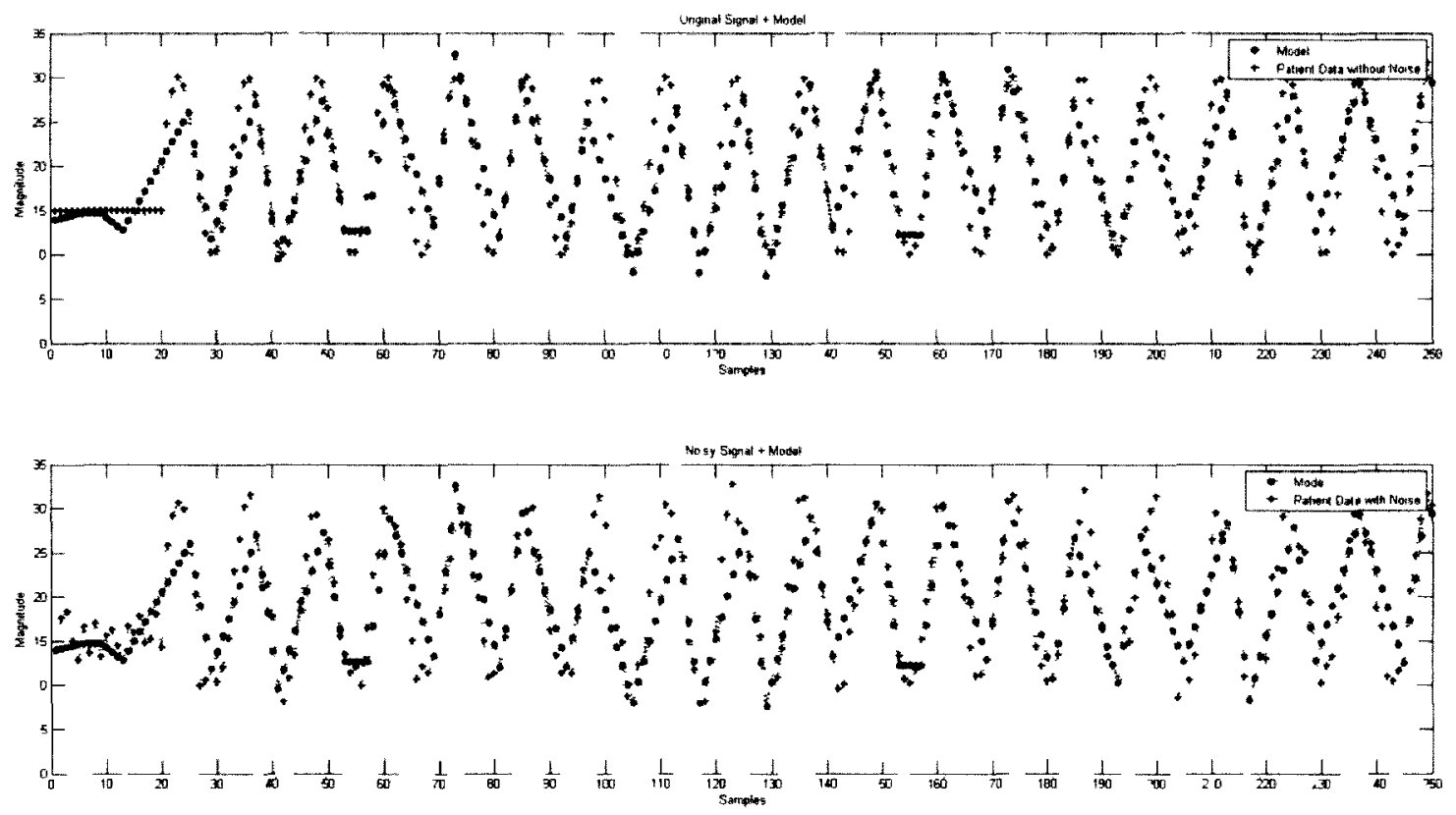

Figure E.7: Plot 2 - Noise 5\%
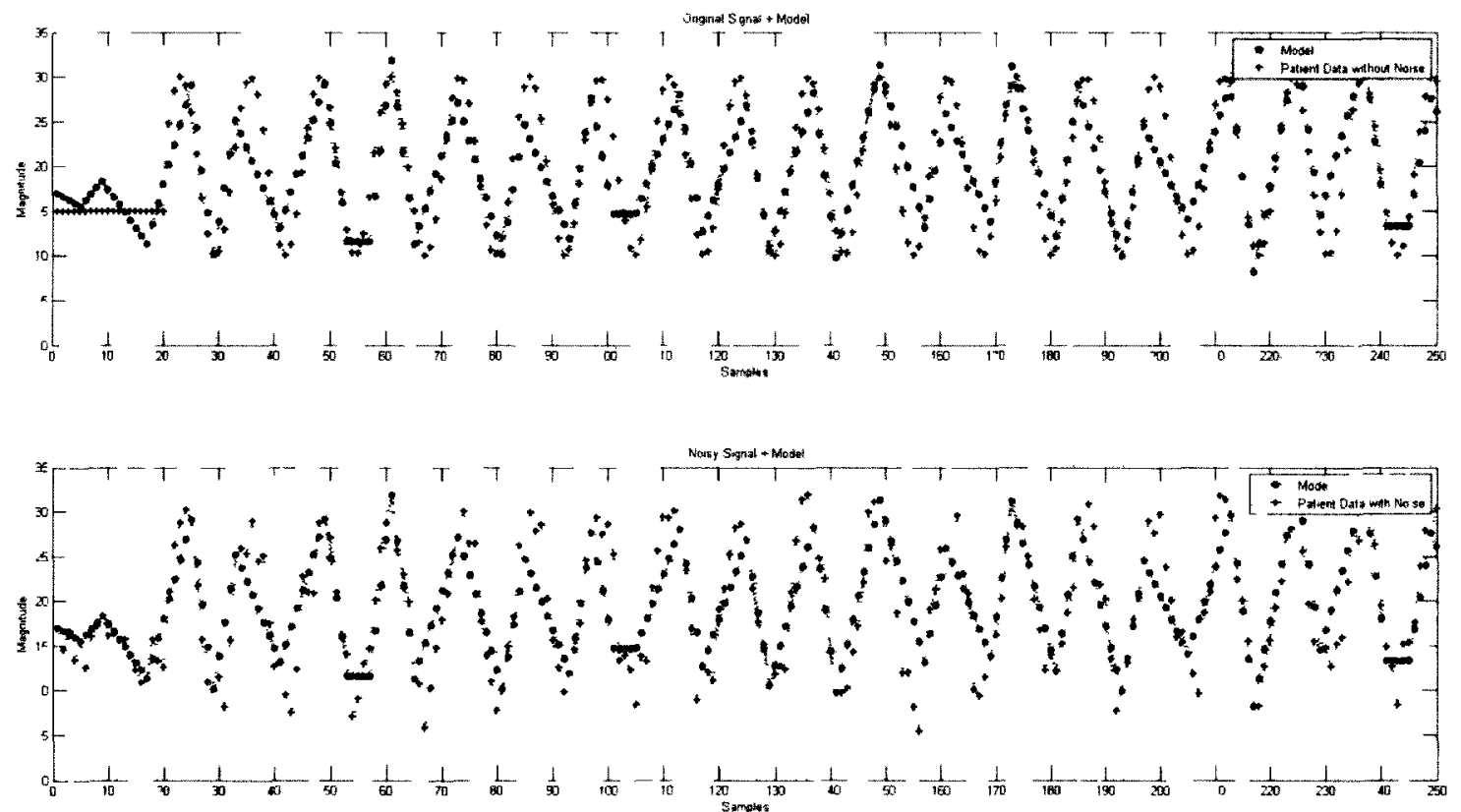

Figure E.8: Plot 2 - Noise 7\% 

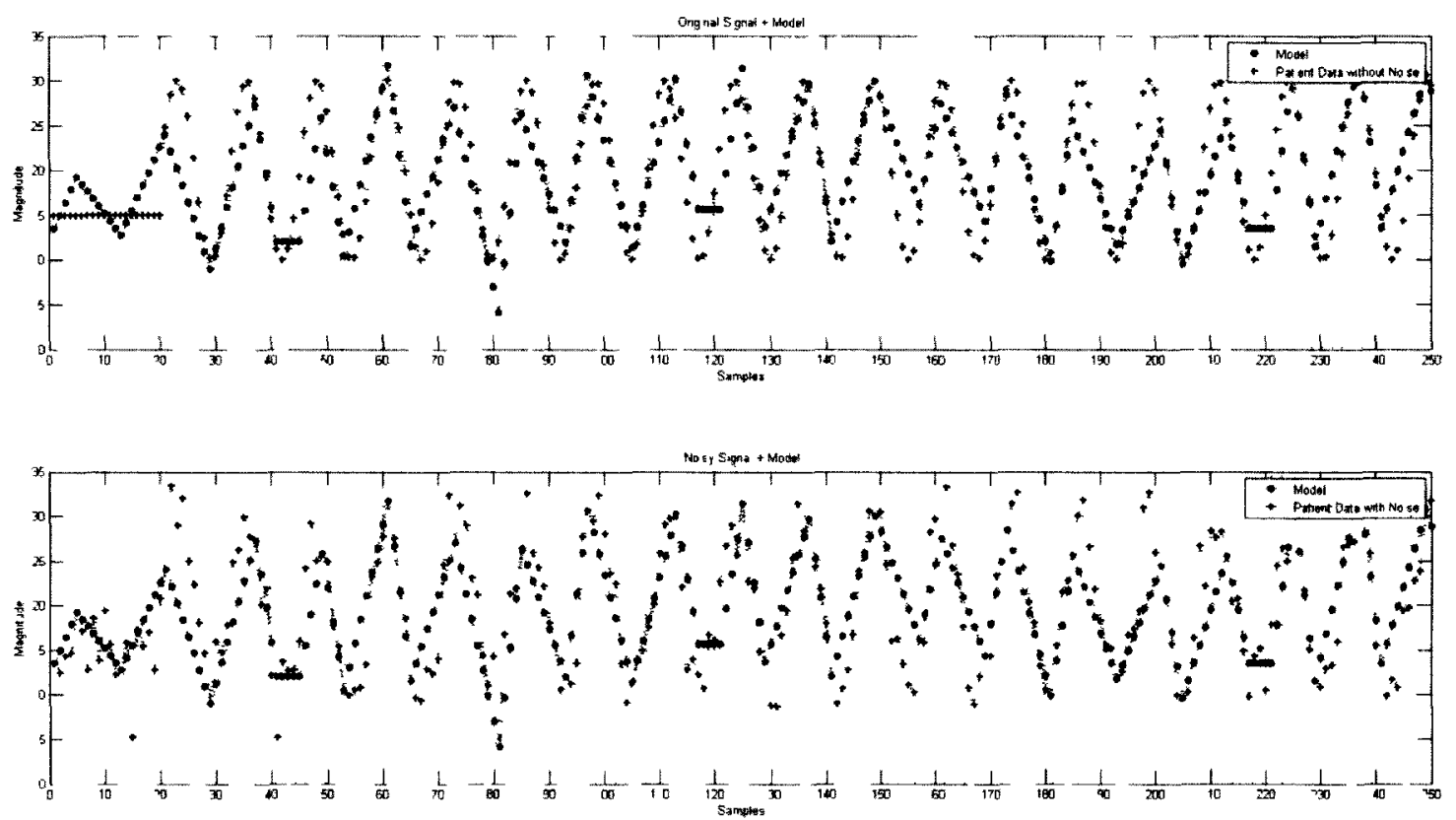

Figure E.9: Plot 2 - Noise 9\%
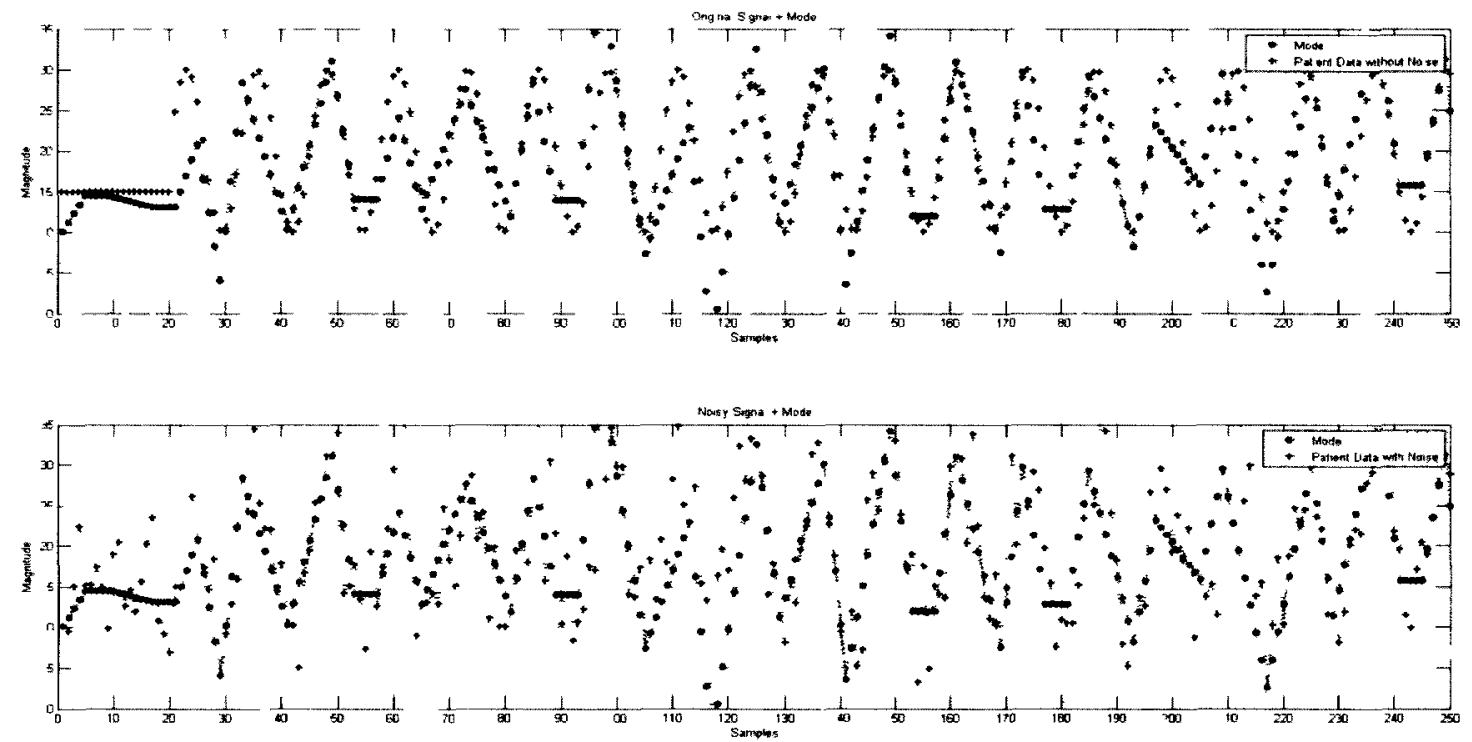

Figure E.10: Plot 2 - Noise 17\% 

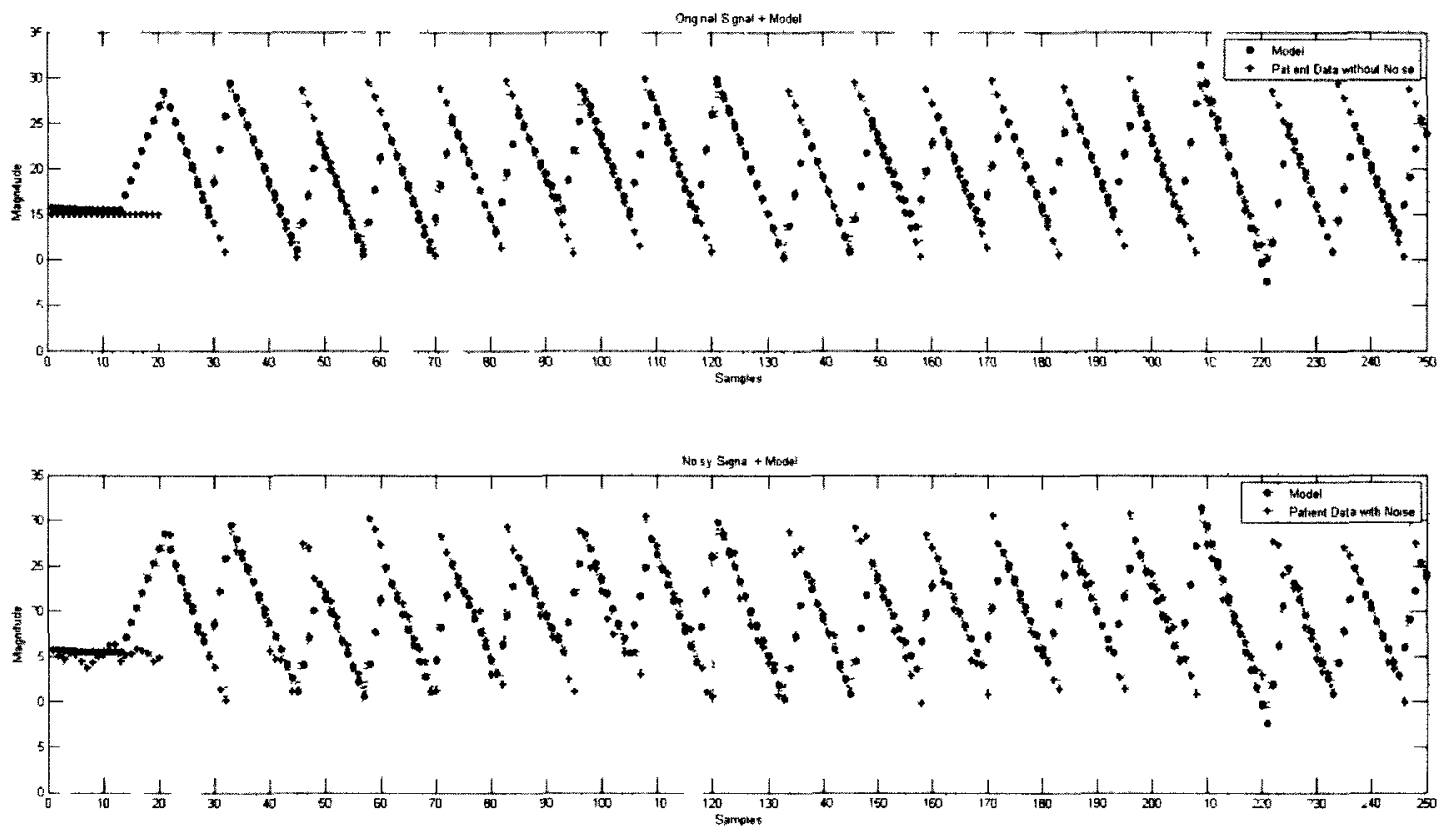

Figure E.11: Plot 3 - Noise 3\%
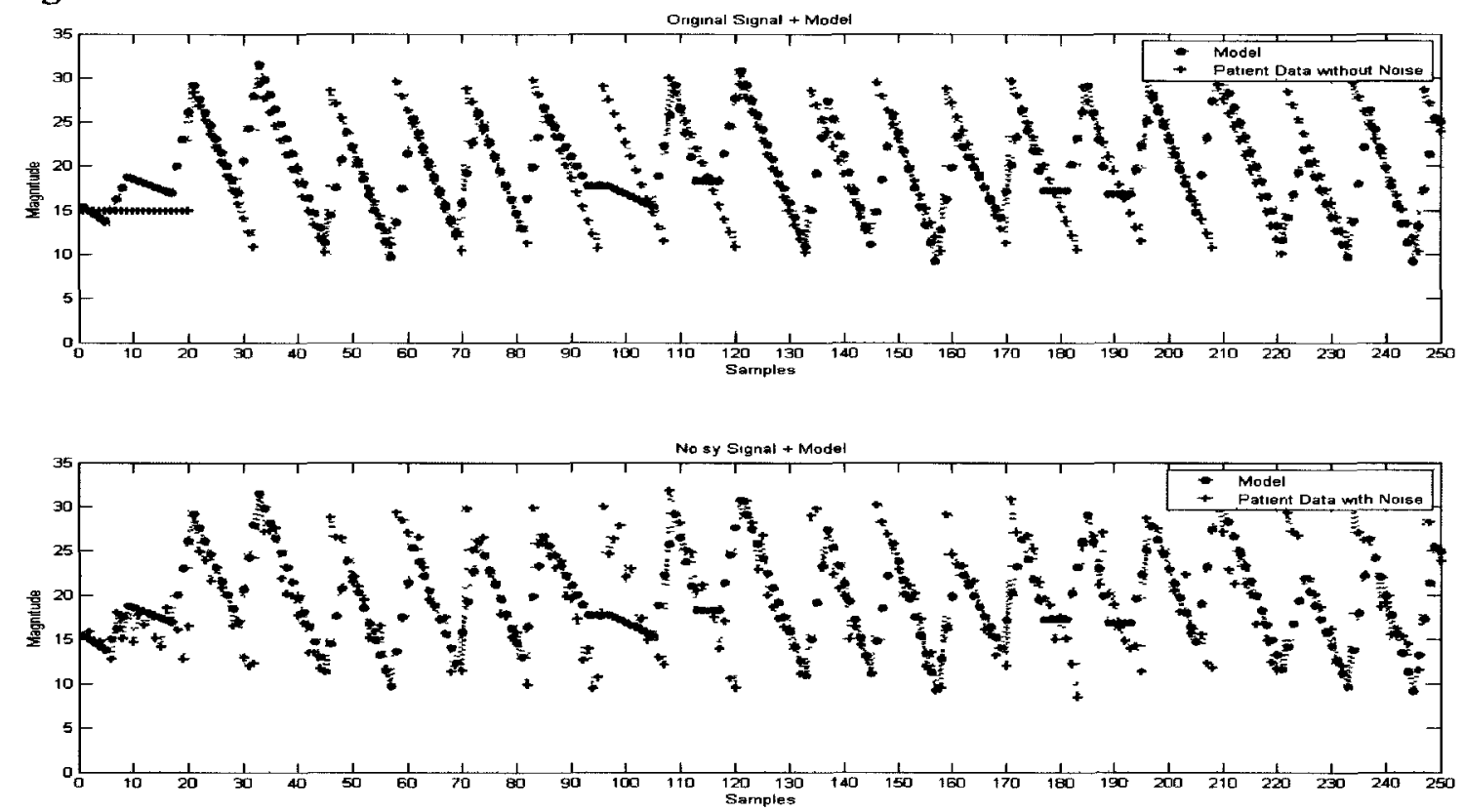

Figure E.12: Plot 3- Noise 5\% 

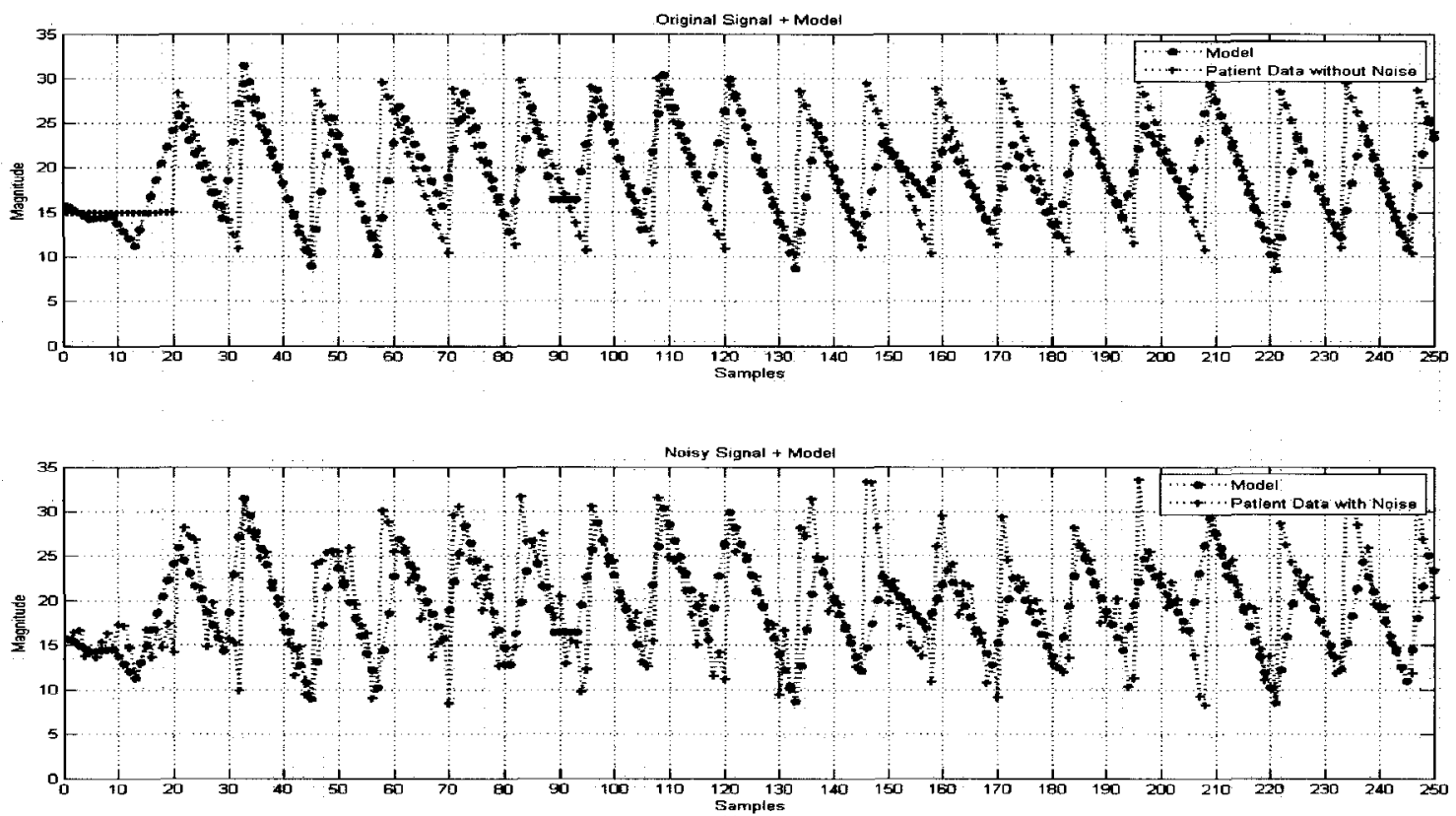

Figure E.13: Plot 3 - Noise 7\%
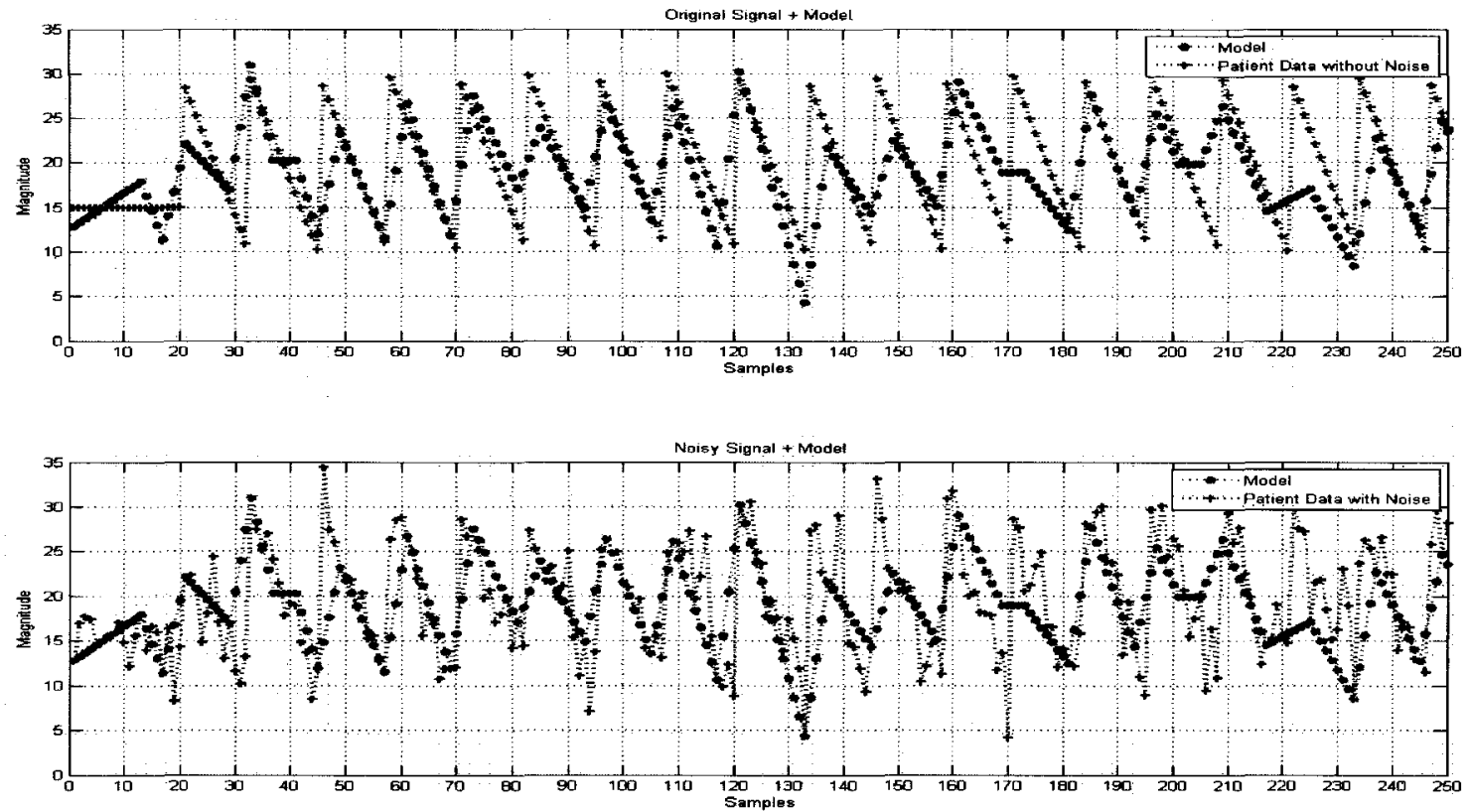

Figure E.14: Plot 3 - Noise 9\% 

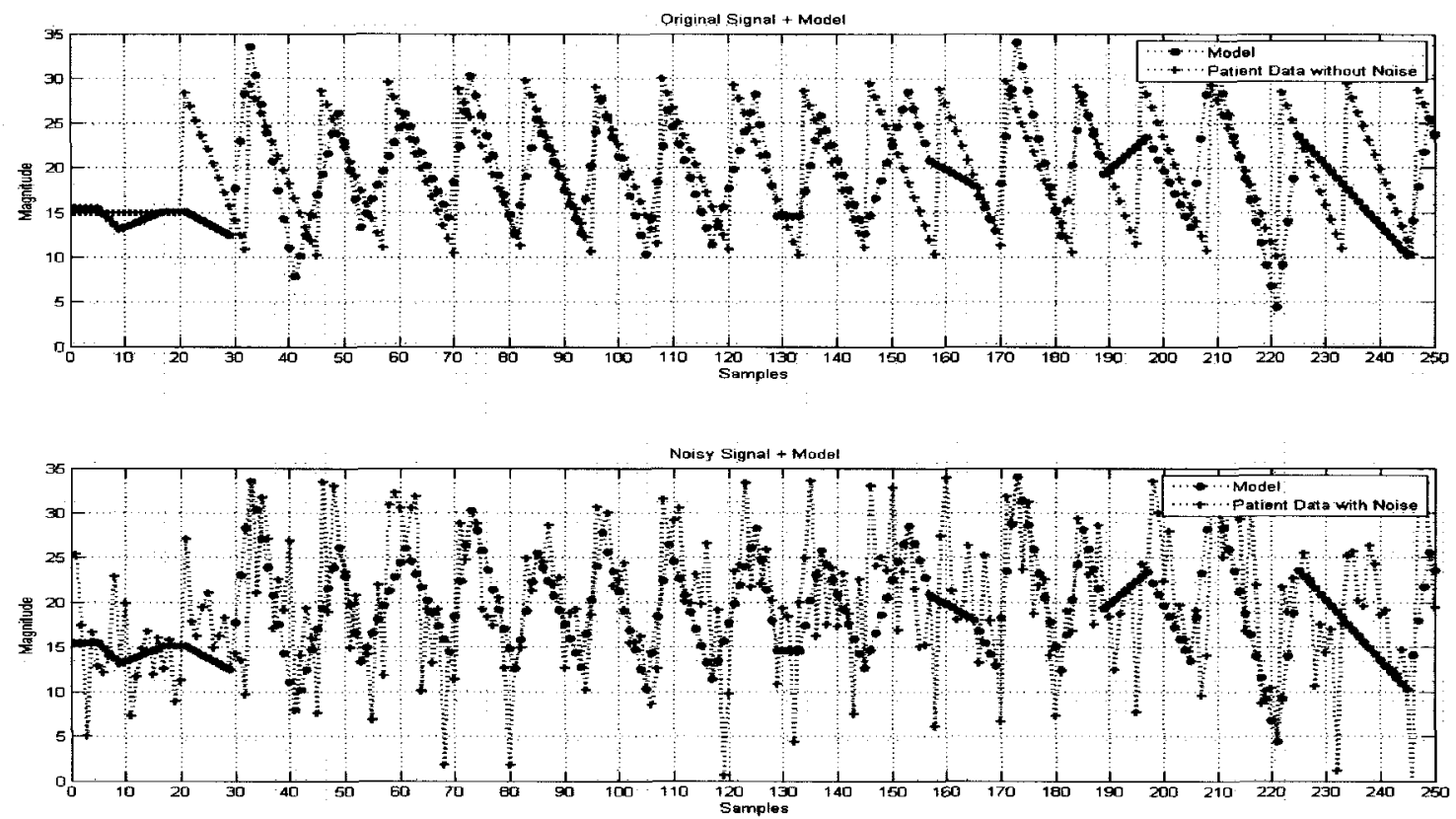

Figure E.15: Plot 3 - Noise 17\%
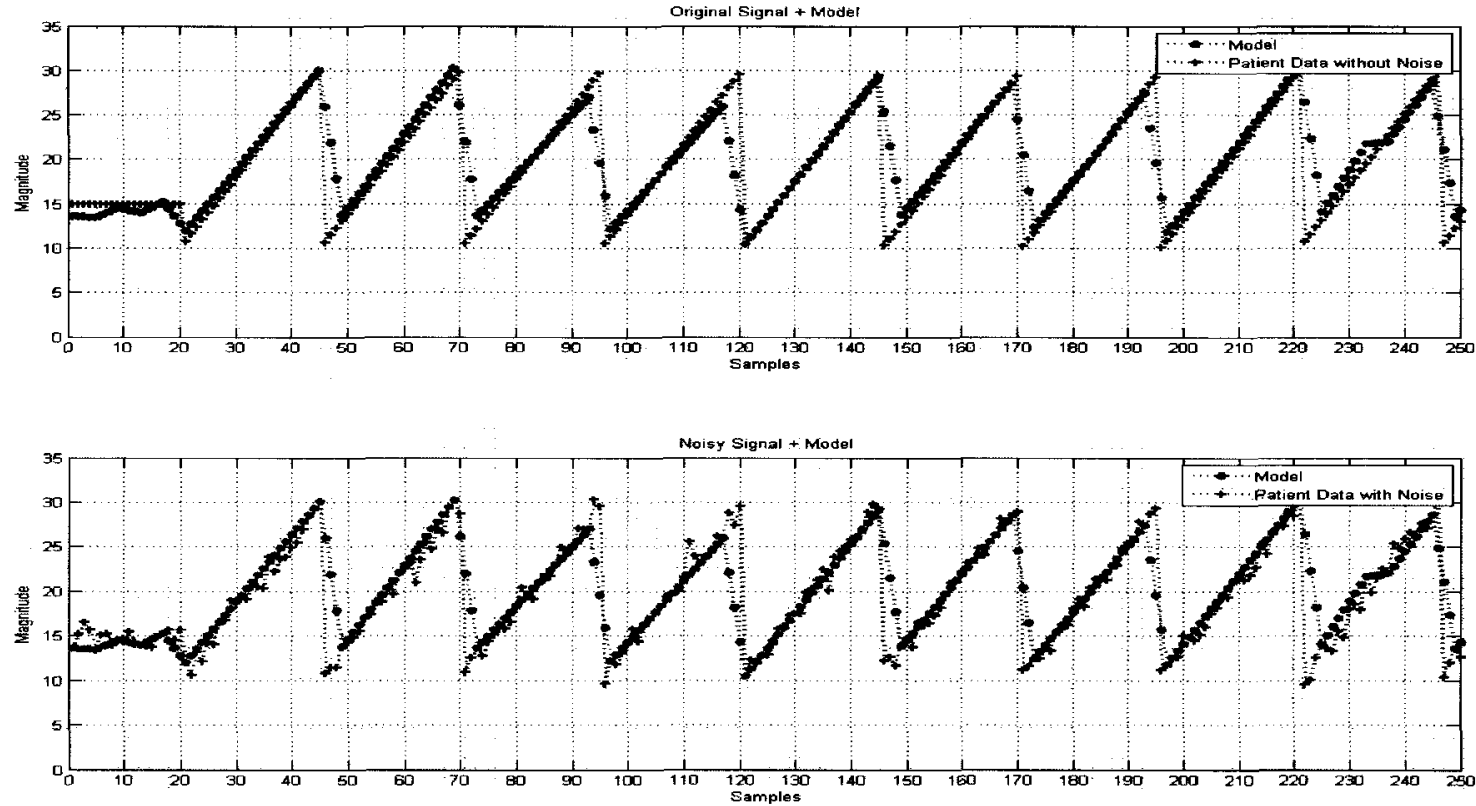

Figure E.16: Plot 4 - Noise 3\% 

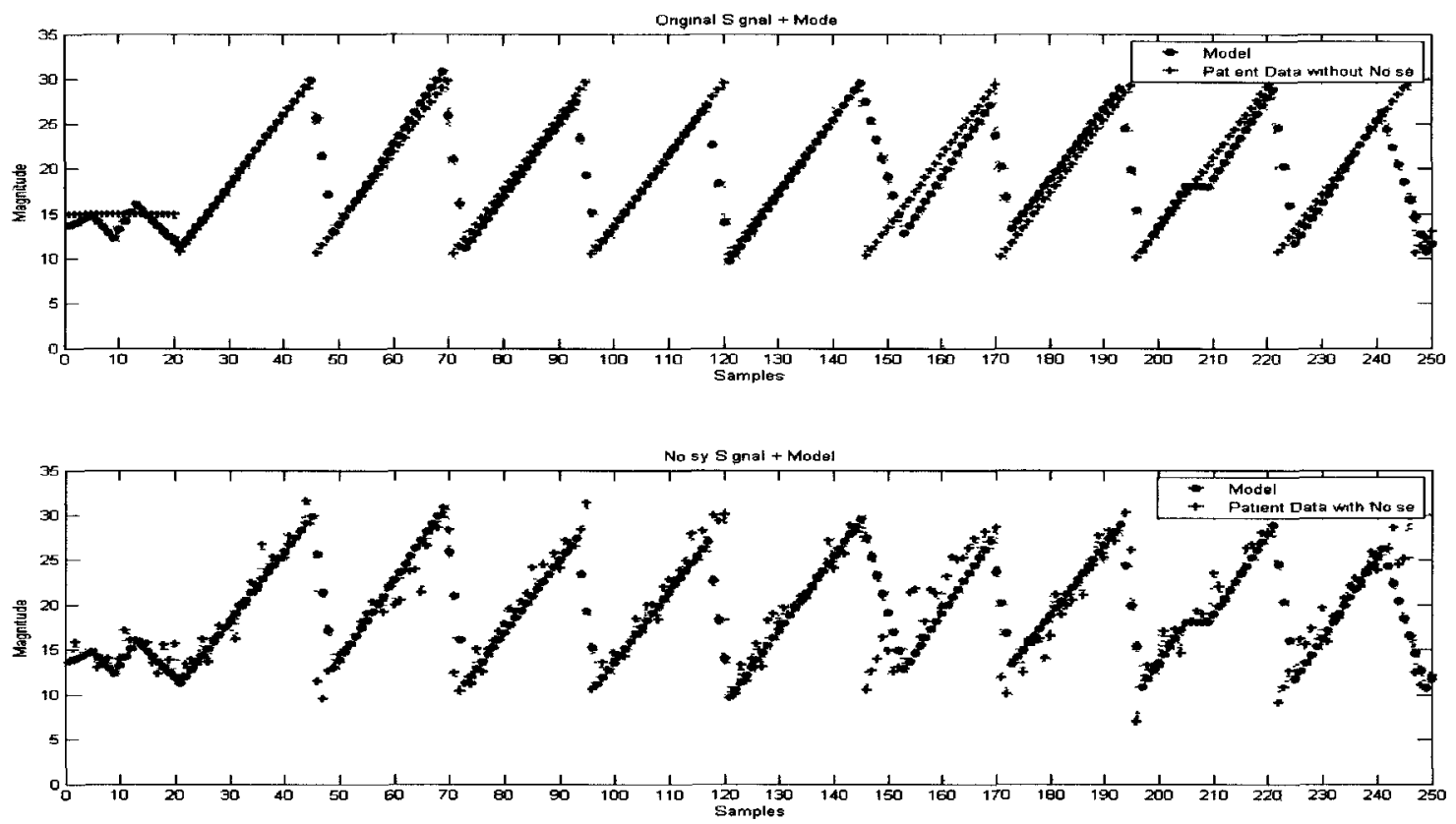

Figure E.17: Plot 4 - Noise 5\%
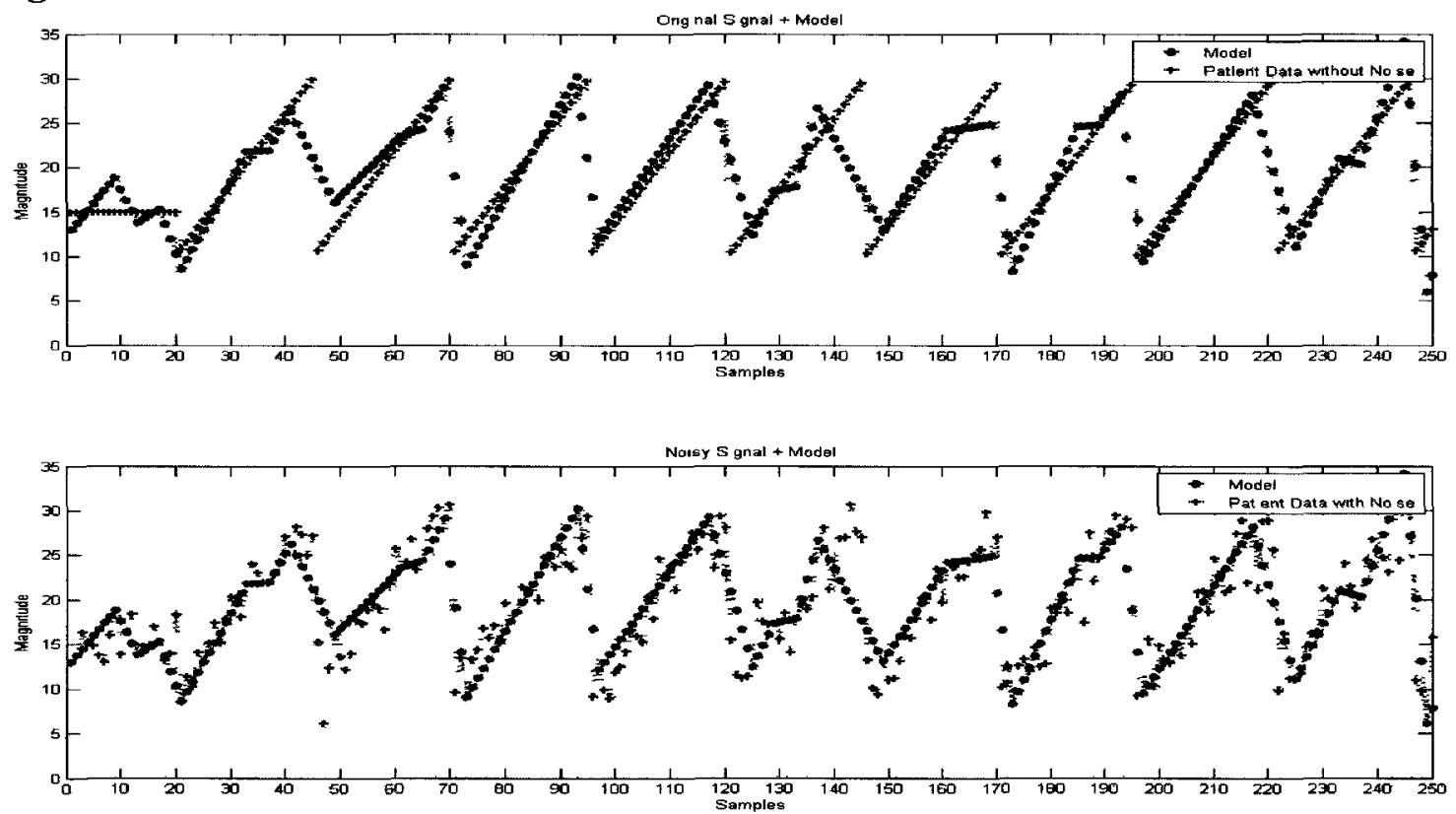

Figure E.18: Plot 4 - Noise 7\% 

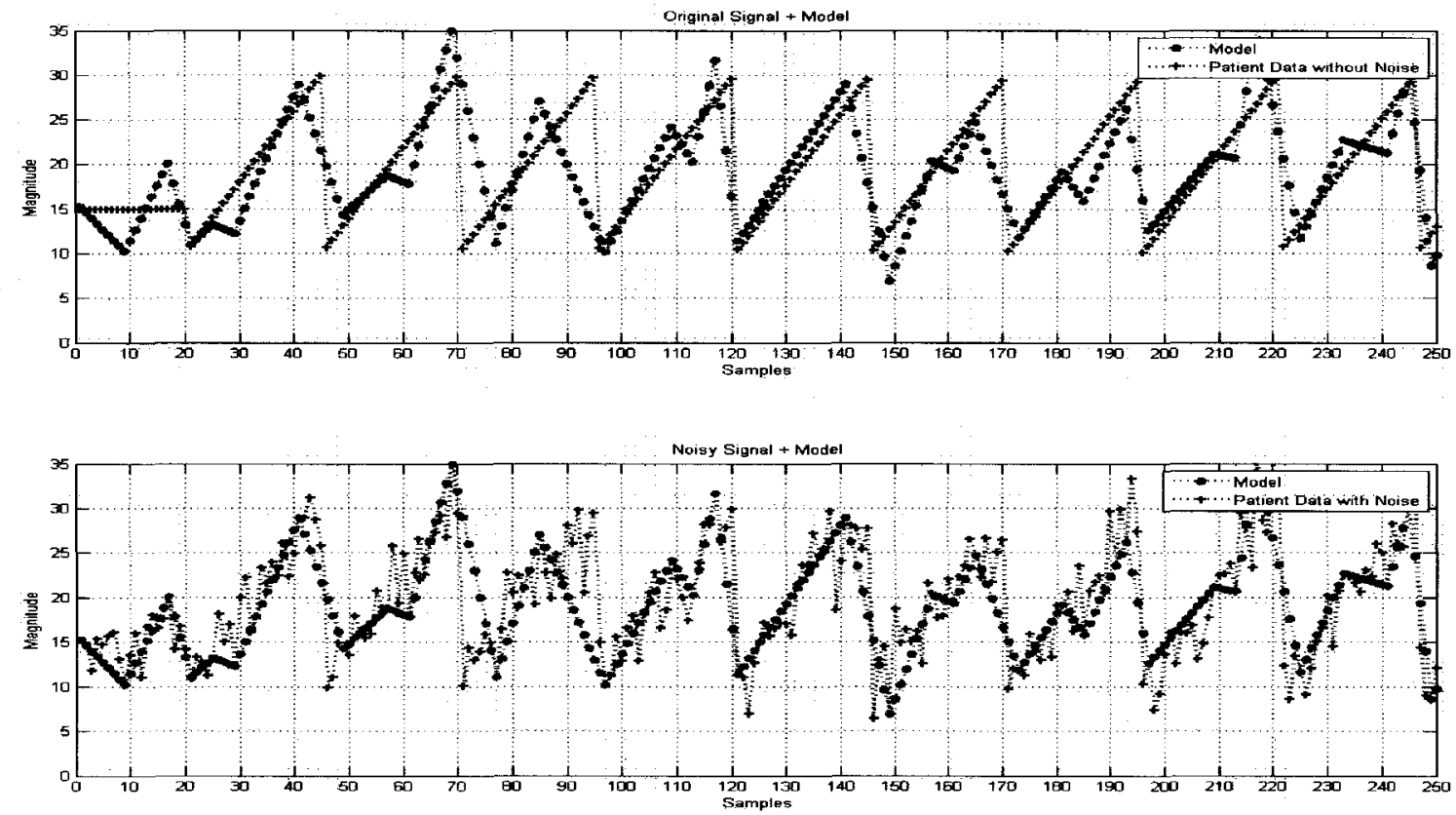

Figure E.19: Plot 4 - Noise 9\%
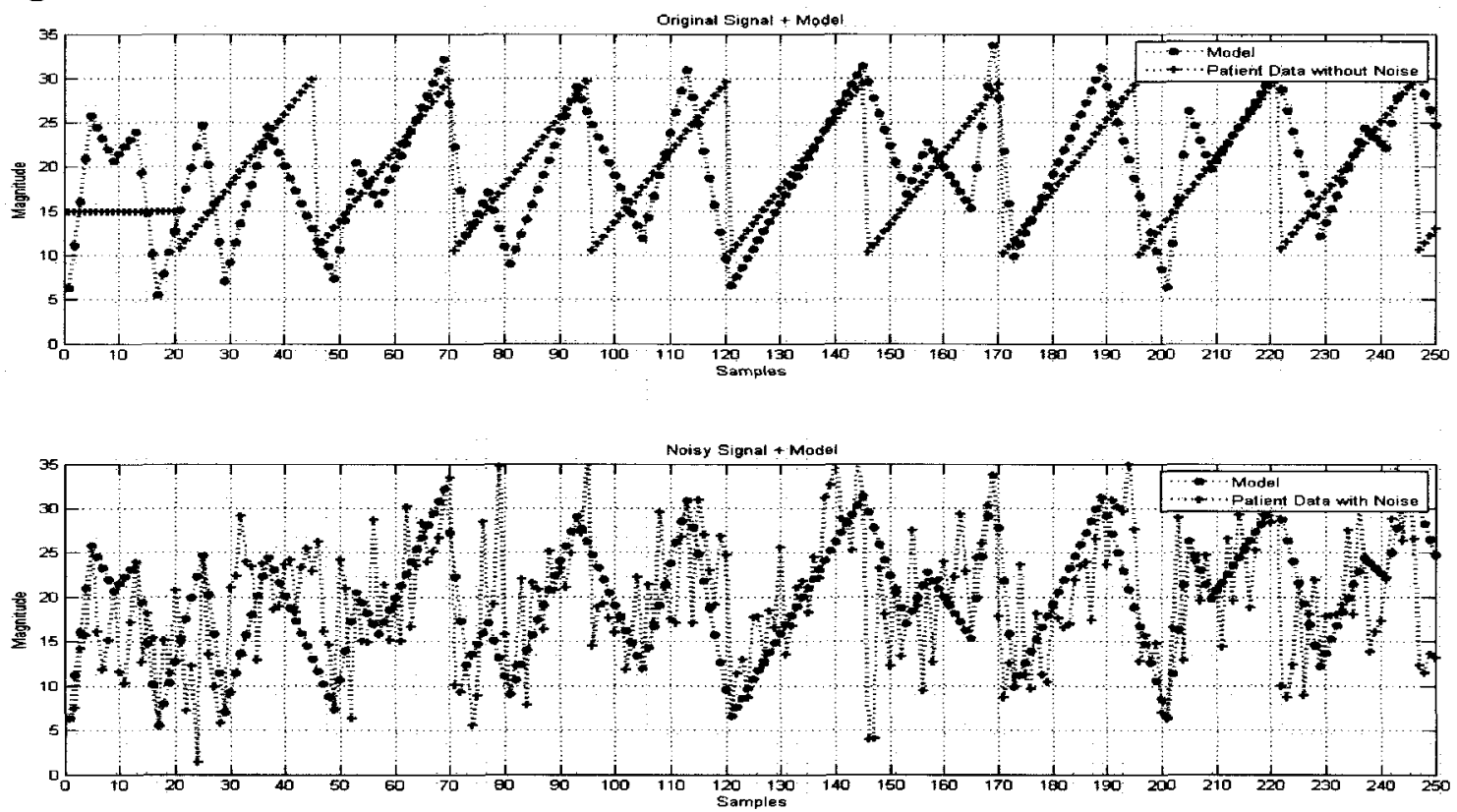

Figure E.20: Plot 4 - Noise 17\% 


\section{Appendix F: Feed-forward Neural Network - Optimal Parameter Plots for Seven Primitives}
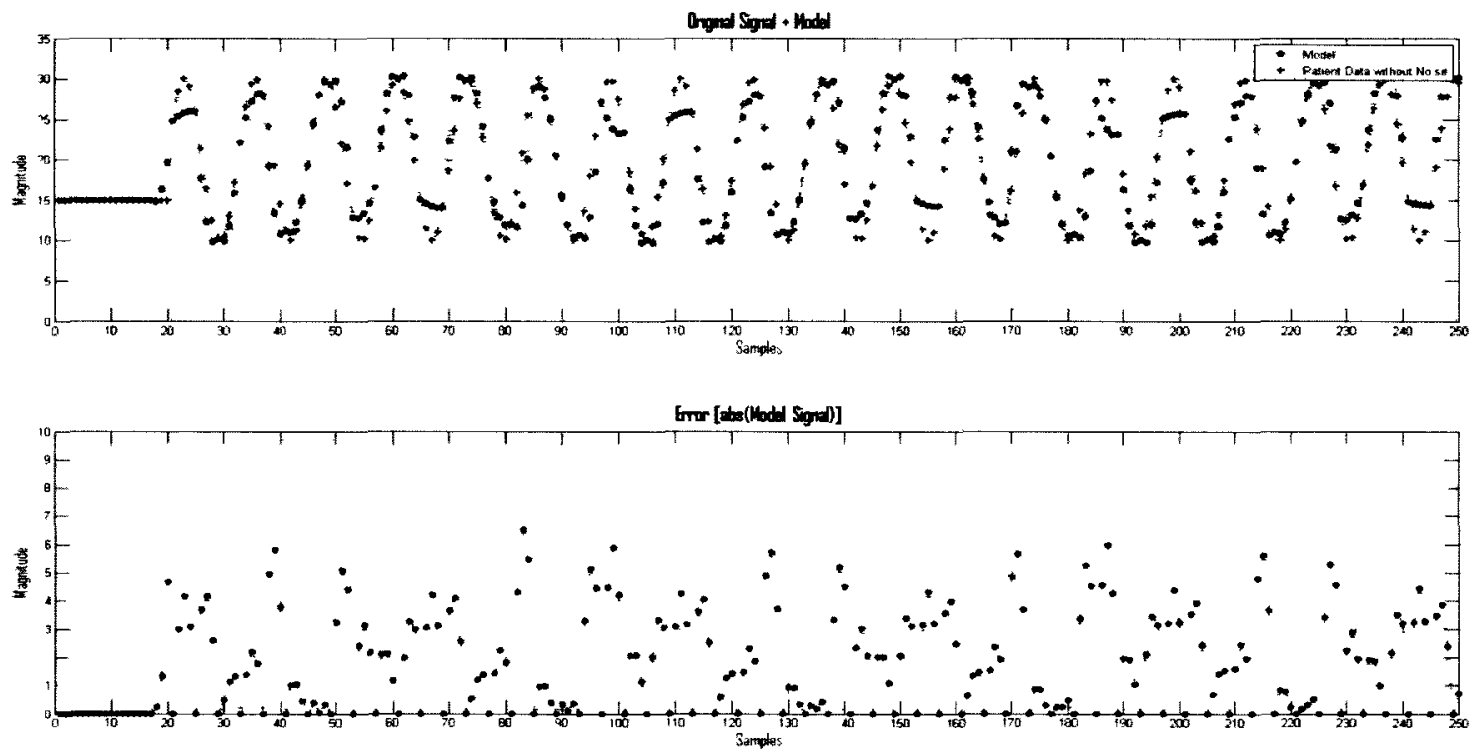

Figure F.1: Plot 2
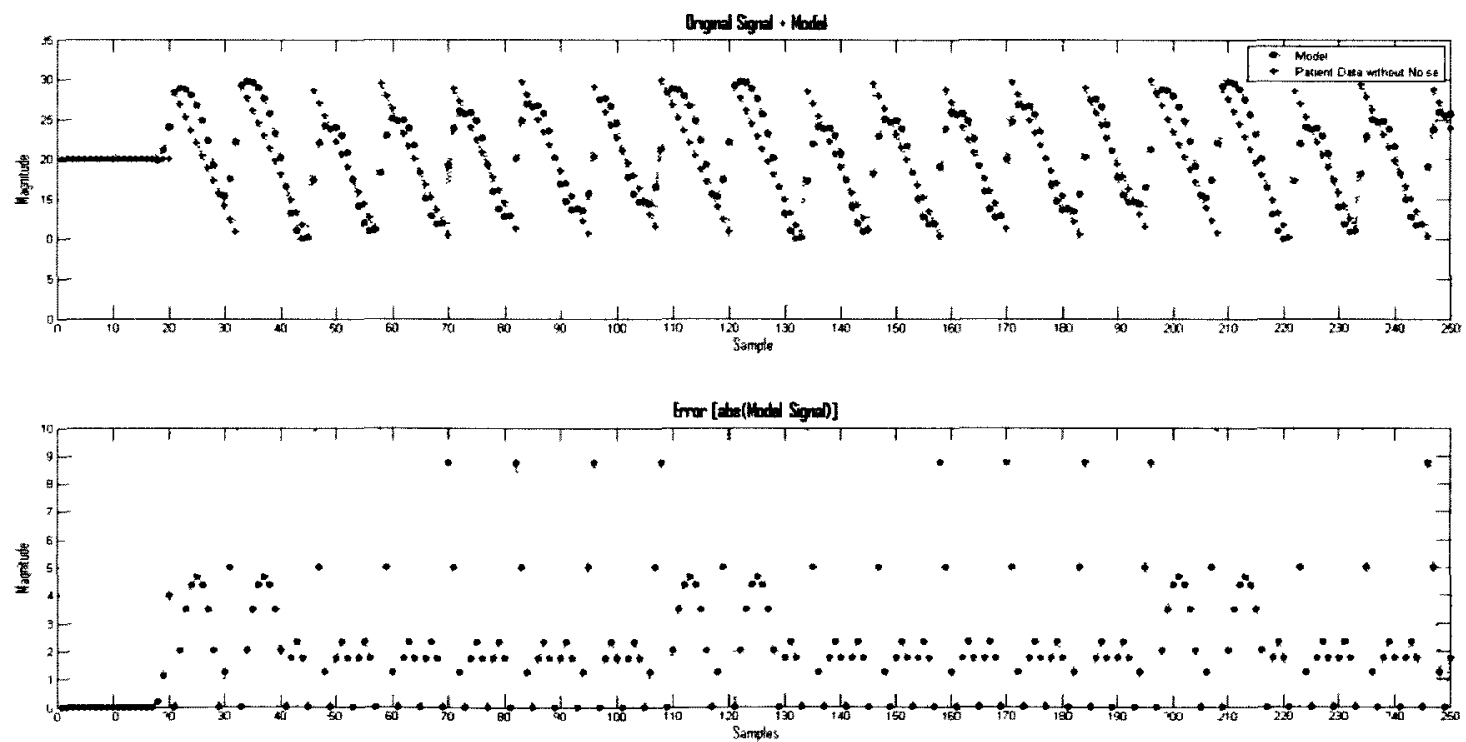

Figure F.2: Plot 3 

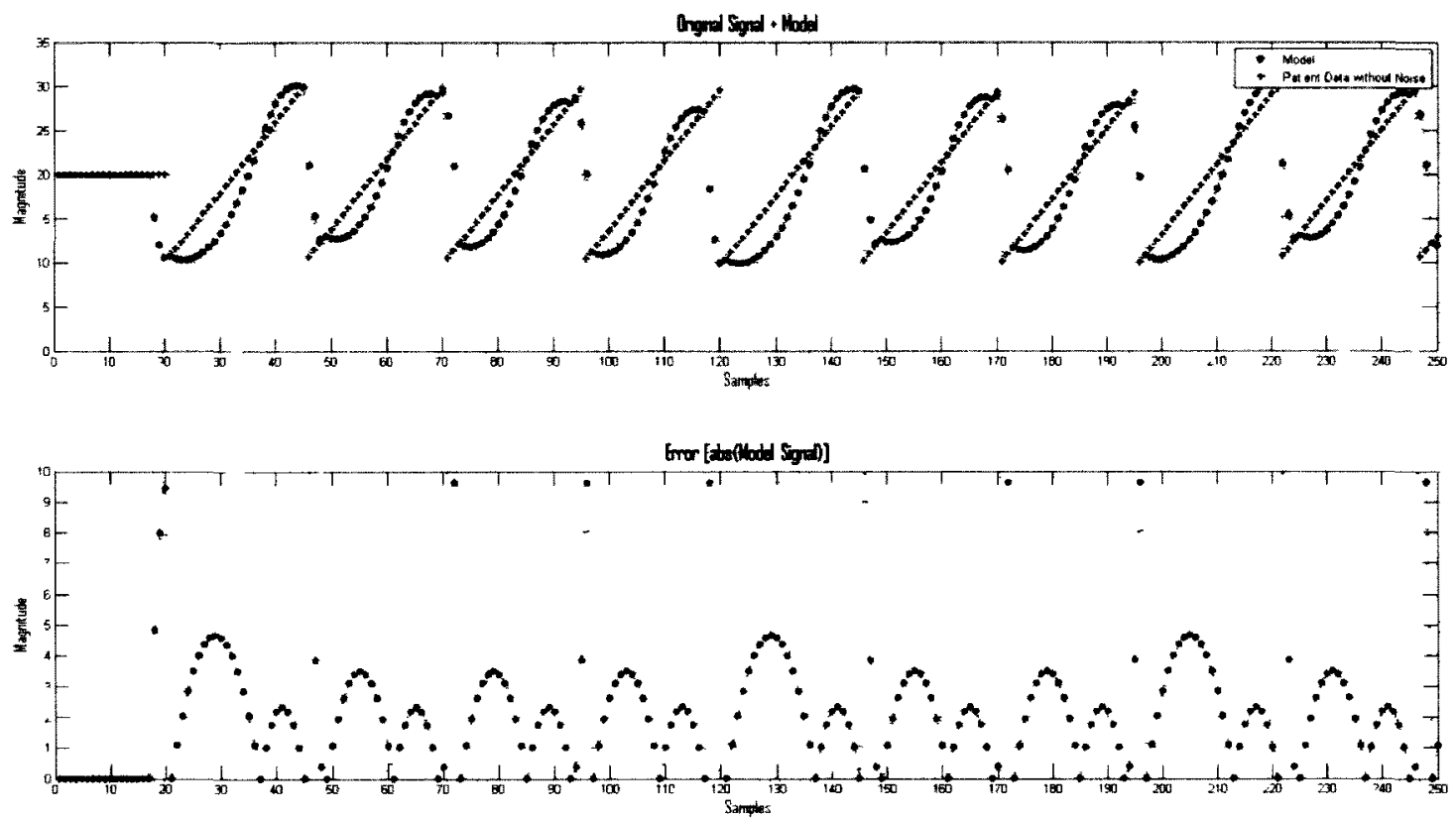

Figure F.3: Plot 4
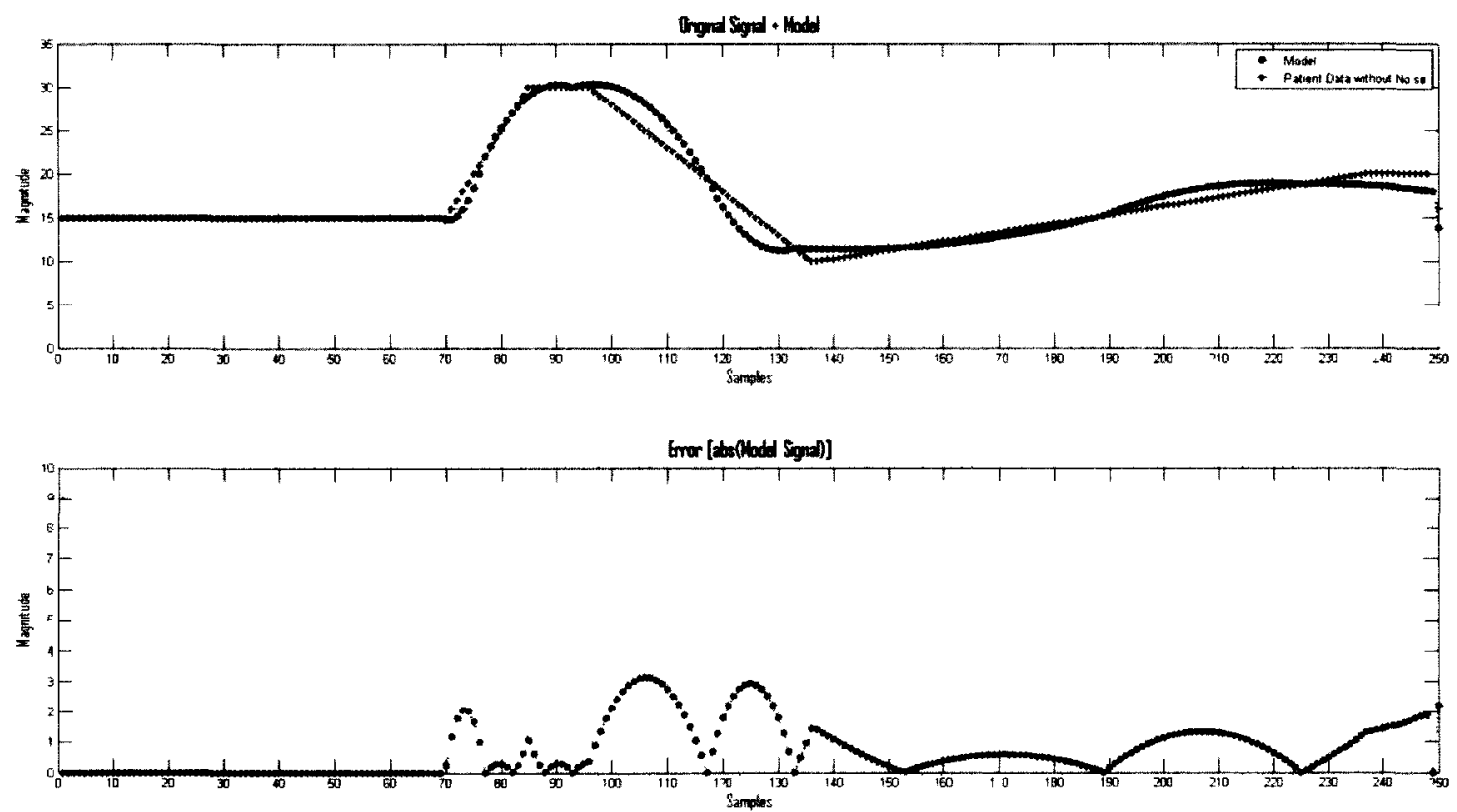

Figure F.4: Plot 5 